\title{
MODELOS PARA PROPORÇÕES COM SUPERDISPERSÃO PROVENIENTES DE ENSAIOS TOXICOLÓGICOS NO TEMPO
}

SILVIA MARIA DE FREITAS

Bacharel em Estatística

Orientadora: Prof. ${ }^{a}$ Dr. ${ }^{a}$ CLARICE GARCIA BORGES DEMÉTRIO

Tese apresentada à Escola Superior de Agricultura "Luiz de Queiroz", Universidade de São Paulo, para obtenção do título de Doutor em Agronomia, Área de Concentração: Estatística e Experimentação Agronômica.

PIRACICABA

Estado de São Paulo - Brasil

Maio - 2001 
SILVIA MARIA DE FREITAS. Modelos para Proporções com Superdispersão para Dados provenientes de Ensaios Toxicológicos no Tempo

\begin{tabular}{|c|c|c|c|c|}
\hline p. & ítem & linha & onde se lê & leia-se \\
\hline 1 & 1.0 & 10 & 10 ton./ & $10 \mathrm{t} /$ \\
\hline 3 & 1.0 & 10 & verificoue & verificou-se \\
\hline 3 & 1.0 & 28 & (1974) e Zeger & (1974). Zeger \\
\hline 5 & 2.0 & 4 & agronômoca & agronômica \\
\hline 7 & 2.0 & 29 & $\left(L D_{50}\right)$ & $\left(E D_{50}\right)$ \\
\hline 14 & 2.0 & 11 & medidas. & medidas repetidas. \\
\hline 17 & 3.0 & 8 & $\begin{array}{l}\text { apresentados os diagramas de dispersão } \\
\text { para as proporções de }\end{array}$ & as proporções de \\
\hline 18 & 3.0 & 1 & 11 a 16$)$ & 73 a 78$)$ \\
\hline 18 & 3.0 & 1 & apresentados os diagramas de dispersão para & apresentadas \\
\hline 20 & Tabela 1 e 2 & título & após & durante \\
\hline 21 & 4.0 & 4 & $D=8$ ou 6$)$ & $D=9$ ou 7$)$ \\
\hline 21 & Tabela 3 & título & insetos & indivíduos \\
\hline 22 & Tabela 4 & título & insetos & indivíduos \\
\hline 22 & Tabela 4 & corpo & $m$ &,$D$ \\
\hline 25 & 4.1 & 4 & $\hat{b e t} a_{2 i}$ & $\hat{\beta}_{2 i}$ \\
\hline 41 & 4.8 & 1 & evaluated at current estimate of & $\begin{array}{l}\text { atualizado na estima- } \\
\text { tiva corrente de }\end{array}$ \\
\hline 42 & 5.1 & 12 & dos cupins & com o cupim \\
\hline 43 & 5.1 & 7 & Figura $9(c, d)$ & Figura $9(b)$ \\
\hline 43 & 5.1 & 9 & predictor & preditor \\
\hline 70 & 5.2 & 11 & REMI & REMII \\
\hline
\end{tabular}


Dados Internacionais de Catalogação na Publicação (CIP) DIVISÃO DE BIBLIOTECA E DOCUMENTAÇÃO - Campus "Luiz de Queiroz"/USP

\author{
Freitas, Silvia Maria de \\ Modelos para proporçōes com superdispersão provenientes de ensaios toxicológicos no \\ tempo / Silvia Maria de Freitas. - - Piracicaba, 2001.
}

$124 \mathrm{p}$.

Tese (doutorado) - Escola Superior de Agricultura Luiz de Queiroz, 2001. Bibliografia.

1. Controle biológico 2. Ensaio biológico 3. Estatística aplicada 4. Fungo entomopatogênico 5. Modelo matemático I. Título 
Aos meus queridos pais Manoel Messias

e Irene Margarida (in memoriam),

DEDICO

As minhas irmãs Susy e Sissi, ao meu irmão Messias Filho, meu cunhado José Paulo e à minha querida sobrinha Raísa pela força de ontem, hoje e de sempre, OFEREÇO 


\section{Agradecimentos}

A $\operatorname{Prof}^{a}$. Dr ${ }^{a}$. Clarice Garcia Borges Demétrio, a orientação, a amizade, o carinho e o constante estímulo.

Ao Professor John Philipe Hinde a supervisão, a amizade, o carinho, o estímulo e a disponibilidade pela oportunidade de desenvolver boa parte deste trabalho junto ao Departamento de Estatística da School of Mathematical Science.

À Fundação Coordenação de Aperfeiçoamento de Pessoal de Nível Superior (CAPES) a bolsa de estudos concedida.

Aos Professores e funcionários do Departamento de Ciências Exatas da ESALQ/USP, o apoio.

Aos Professores e funcionários do Departamento de Estatística e Matemática Aplicada da Universidade Federal do Ceará (UFC), o incentivo e o apoio em todas as etapas do trabalho.

Aos colegas de curso o companheirismo e a amizade.

Aos amigos antigos e aos novos o incentivo, a amizade e o carinho eterno. Em especial às minhas amigas de turma: Cecília, Denise, Leda e Jeanete a força em todos os momentos por quais passamos.

Ao Prof. Dr. Sérgio Alves Batista a gentileza de ter cedido os dados utilizados neste trabalho.

Ao John, à Kathie e à Naratip a maravilhosa acolhida e a amizade em

Exeter.

À Roseli, Silvio, Gláucia e Clemente, os bons momentos passados juntos.

Ao Maurício e ao André o apoio, a paciência e a colaboração.

Ao Guilbert e ao Vagner por tudo, o meu carinho e amizade eterna.

A Deus a força de caminhar sempre em frente, mesmo durante os caminhos tortuosos pelos quais me deparei. 


\section{SUMÁRIO}

Página

LISTA DE FIGURAS

$\mathbf{v}$

LISTA DE TABELAS vii

1 INTRODUÇÃO 1

2 REVISÃO DE LITERATURA

3 MATERIAL $\quad 16$

4 MÉTODOS $\quad 21$

4.1 Modelo multinomial . . . . . . . . . . . . . . 22

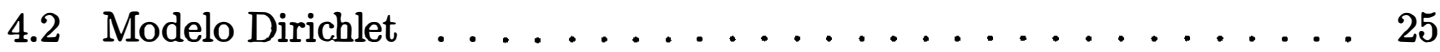

4.3 Modelo Dirichlet - multinomial . . . . . . . . . . 26

4.4 Modelo multinomial acumulado . . . . . . . . . . . . . 28

4.5 Modelo Dirichlet - multinomial acumulado . . . . . . . . . . 29

4.6 Modelos de efeito aleatório . . . . . . . . . . . . . 29

4.6.1 Modelo de efeito aleatório no intercepto - REMI . . . . . . . 30

4.6.2 Modelo de efeitos aleatórios no intercepto e coeficiente angular - REMII ........................ 32

4.7 Equações de estimações generalizadas . . . . . . . . . . . . . 35

4.8 Estimação dos parâmetros dos modelos para os ensaios com os cupins e os ácaros . . . . . . . . . . . . . . . . . . 38

5 RESULTADOS E DISCUSSÃO 42

5.1 Resultados para o ensaio com o cupim H. tenuis: . . . . . . . . . 42

5.2 Resultados para o ensaio com o ácaro T. urticae: . . . . . . . . 68 


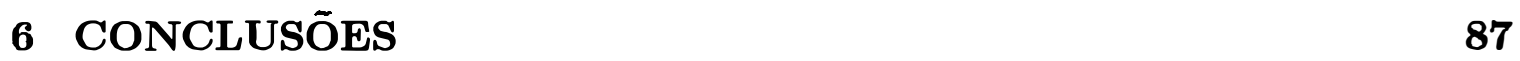

$\begin{array}{ll}\text { REFERENCIAS BIBLIOGRAFICAS } & 90\end{array}$

$\begin{array}{ll}\text { APENNDICE } 1 & 98\end{array}$ 


\section{LISTA DE FIGURAS}

Página

1 Proporção da mortalidade acumulada durante os 8 dias, para vinte isolados do experimento com o cupim H. tenuis. . . . . . . . . . 50

2 Proporção da mortalidade acumulada durante os 8 dias, para vinte isolados do experimento com o cupim $H$. tenuis. . . . . . . . . . 51

3 Proporção da mortalidade acumulada durante os 8 dias, para vinte isolados do experimento com o cupim $H$. tenuis. . . . . . . . . . 52

4 Proporção da mortalidade acumulada durante os 8 dias, para vinte isolados do experimento com o cupim $H$. tenuis. . . . . . . . . . 53

5 Proporção da mortalidade acumulada durante os 8 dias, para vinte isolados do experimento com o cupim $H$. tenuis. . . . . . . . . . . 54

6 Proporção da mortalidade acumulada durante os 8 dias, para vinte isolados do experimento com o cupim $H$. tenuis. . . . . . . . . 55

7 Proporção da mortalidade acumulada durante os 8 dias, para vinte isolados do experimento com o cupim H. tenuis. . . . . . . . . . 56

8 Proporção da mortalidade acumulada durante os 8 dias, para dois isolados do experimento com o cupim H. tenuis. . . . . . . . . . . . 57

9 Diagramas de dispersão do d.p. $\left(\beta_{1}\right)$ e d.p. $\left(\beta_{2}\right)$ para o modelo multinomial $\times$ DM (o), REMI $(\triangle)$ e REMII $(+)$, usando a matriz de variâncias e covariância assintótica (a) e robusta (b). . . . . . . . 58

10 Histogramas de $\rho, \sigma^{2}, \nu^{2}, \tau^{2}$ (com (c), (e) e sem $(d),(f)$ o Isolado 987), $\lambda, L T_{50}$ e $L T_{90}$ e os diagramas de dispersão do $\beta_{1} \times \beta_{2}$ e $\nu^{2} \times \tau^{2}$ (com $(k)$ e sem $(l)$ o Isolado 987$) \ldots \ldots \ldots$

11 Proporção da mortalidade acumulada durante os 6 dias, para quinze isolados do experimento com o ácaro $T$. urticae. . . . . . . . . 73 
12 Proporção da mortalidade acumulada durante os 6 dias, para quinze isolados do experimento com o ácaro T. urticae. . . . . . . . . . 74

13 Proporção da mortalidade acumulada durante os 6 dias, para quinze isolados do experimento com o ácaro T. urticae. . . . . . . . . 75

14 Proporção da mortalidade acumulada durante os 6 dias, para quinze isolados do experimento com o ácaro T. urticae. . . . . . . . 76

15 Proporção da mortalidade acumulada durante os 6 dias, para quinze isolados do experimento com o ácaro T. urticae. . . . . . . . 77

16 Proporção da mortalidade acumulada durante os 6 dias, para cinco isolados do experimento com o ácaro $T$. urticae. . . . . . . . 78

17 Diagramas de dispersão do d.p. $\left(\beta_{1}\right)$ e d.p. $\left(\beta_{2}\right)$ para o modelo multinomial $\times \operatorname{DM}($ (o), REMI $(\triangle)$ e REMII $(+)$, usando a matriz de variâncias e covariância assintótica (a) e robusta (b). . . . . . . 79

18 Histogramas de $\rho, \sigma^{2}, \tau^{2}, \lambda, L T_{50}$ e $L T_{90}$ e os diagramas de dispersão do $\beta_{1} \times \beta_{2}$ e $L T_{50} \times L T_{90} \ldots \ldots \ldots \ldots \ldots \ldots$ 


\section{LISTA DE TABELAS}

Página

1 Mortalidade acumulada de cupins após oito dias de exposição a dois isolados de B. bassiana. . . . . . . . . . . . . . . . . 20

2 Mortalidade acumulada de ácaros após oito dias de exposição a dois isolados de B. bassiana. . . . . . . . . . . . . . . 20

3 Números $\left(y_{i k, j}\right)$ observados de insetos mortos no tempo $j$, para o isolado $i$ nas diferentes repetições. . . . . . . . . . . . . . . . . 21

4 Proporções $\left(\boldsymbol{r}_{i k, j}\right)$ acumuladas observadas de insetos mortos até o tempo $j$, para o isolado $i$ nas diferentes repetições. . . . . . . . . . 22

5 Sumário das estatísticas descritivas para os parâmetros de superdispersão dos modelos DM, REMI e REMII, nos quais a superdispersão varia entre os isolados, considerando-se os dados das Tabelas 2 a $9 . \quad$. 44

6 Estimativas dos parâmetros da regressão e dos parâmetros de superdispersão para o ensaio com os cupins. . . . . . . . . . . . . 46

7 Estimativas dos desvios padrões (d.p.) das estimativas dos parâmetros do modelo de regressão calculadas através da matriz de variâncias e covariâncias assintótica, para o ensaio com os cupins. . . . . . . . . 60

8 Estimativas dos desvios padrões (d.p.) das estimativas dos parâmetros do modelo de regressão calculadas através da matriz de variâncias e covariâncias robusta, para o ensaio com os cupins. . . . . . . . . .

9 Sumário das estatísticas descritivas para os parâmetros de superdispersão dos modelos DM, REMI e REMII, nos quais a superdispersão varia entre os isolados, considerando-se o ensaio com os ácaros. . . . . 70

10 Estimativas dos parâmetros da regressão e dos parâmetros de superdispersão para o ensaio com os ácaros. 
11 Estimativas dos desvios padrões (d.p.) das estimativas dos parâmetros do modelo de regressão calculado através da matriz de variâncias e covariâncias assintótica, para o ensaio com os ácaros. . . . . . . . . .

12 Estimativas dos desvios padrões (d.p.) das estimativas dos parâmetros do modelo de regressão calculado através da matriz de variâncias e covariâncias robusta, para o ensaio com os ácaros. . . . . . . . . . 84 


\title{
MODELOS PARA PROPORÇÕES COM SUPERDISPERSĀO PROVENIENTES DE ENSAIOS TOXICOLÓGICOS NO TEMPO
}

\author{
Autora: SILVIA MARIA DE FREITAS \\ Orientadora: Prof. ${ }^{a}$ Dr. $^{a}$ CLARICE GARCIA BORGES DEMÉTRIO
}

\section{RESUMO}

O controle biológico de pragas utiliza agentes entomopatogênicos ou patógenos (vírus, fungos, bactérias, parasitas e nematóides) para controlar ou eliminar uma população de peste. Este método é uma alternativa para métodos tradicionais e está se tornando de interesse crescente em função da proibição do uso de muitos pesticidas químicos, e também porque é ecologica e economicamente atraente.

Nos experimentos em toxicologia com esses agentes, é comum o aparecimento de resposta do tipo mortalidade acumulada em um grupo de insetos, medida em vários pontos no tempo (dias, semanas etc). Consequentemente, os dados são em parte longitudinais, desde que a mortalidade acumulada (para cada grupo) é modelada como uma função do tempo e das covariáveis em cada nível do grupo. O problema de ajustar mortalidade acumulada como uma função do tempo para dados agrupados envolve a modelagem de uma resposta multinomial ao longo do tempo. Um aspecto adicional aqui é a possibilidade de uma variação extra-multinomial (superdispersão) que pode surgir em função do uso dos grupos como unidades experimentais. Como consequência por não se considerar a superdispersão tem-se a subestimação dos erros padrões das estimativas dos coeficientes da regressão, levando a conclusões incorretas dos efeitos de tratamentos e intervalos de confiança muito estreitos (Hinde e Demétrio, 1998a, b).

Para esse tipo particular de dados, existem poucos métodos e modelos apropriados que podem ser aplicados. Podem ser citados as técnicas de análise 
de sobrevivência (Petkau \& Sitter, 1989) e modelos lineares generalizados ordinais (McCullagh, 1980; Glonek \& McCullagh, 1995). Porém, estes procedimentos são questionáveis na presença de variabilidade extra-multinomial nos dados.

$\mathrm{O}$ uso das equações de estimação generalizadas (EEG) de Liang \& Zeger (1986) veio como uma versão multivariada da quase verossimilhança (McCullagh \& Nelder, 1989) para o ajuste de modelos lineares generalizados para dados agrupados. Não é necessária a identificação completa do modelo probabilístico, mas apenas a especificação dos dois primeiros momentos do vetor de resposta para cada agrupamento, definindo a relação funcional entre a média e a variância. Não existe necessidade de especificar completamente a verossimilhança, o que muitas vezes é intratável para dados que não tenham distribuição Gaussiana, até mesmo quando são feitas suposições adicionais. Para dados com distribuição Gaussiana os primeiro e segundo momentos identificam completamente a verossimilhança, em caso contrário fazem-se necessárias suposições adicionais sobre momentos de ordem superior a dois.

Neste trabalho foi utilizada a metodologia de modelos lineares generalizados para dados multinomiais na modelagem de dados agrupados com superdispersão, seguindo a metodologia proposta por O'Hara Hines \& Lawless (1993). Usouse uma função de ligação logística, considerando o dia como uma variável explicativa, em todos os modelos considerados. O primeiro modelo utilizado foi o modelo multinomial padrão acumulado. O segundo modelo, uma extensão do modelo beta-binomial, utiliza uma distribuição multinomial para a variável resposta e uma distribuição Dirichlet para o vetor de probabilidades, levando a uma distribuição composta Dirichlet-multinomial. O terceiro modelo incorpora um efeito aleatório ao preditor linear produzindo um efeito diferenciado no intercepto da regressão em cada amostra multinomial. O modelo final inclui efeitos aleatórios no intercepto e no coeficiente angular da regressão. 


\title{
OVERDISPERSION MODELS FOR PROPORTIONS FROM TOXICOLOGICAL BIOASSAYS OVER TIME
}

\author{
Author: SILVIA MARIA DE FREITAS \\ Adviser: Prof. ${ }^{a}$ Dr. ${ }^{a}$ CLARICE GARCIA BORGES DEMÉTRIO
}

\section{SUMMARY}

Biological pest control uses entomopathogenic agents or pathogens (viruses, fungi, bacteria, parasites and nematodes) to control or eliminate a pest population. These biological control methods are an alternative to traditional methods and are becoming of increased interest because of the cancelation of many chemical pesticide registrations, and also because they are ecologically and economically attractive.

In toxicology experiments for these agents, it is common to use a response such as the cumulative mortality in a group (cluster) of insects measured at various time points (days) during the course of the experiment. Hence, the data is partly longitudinal since the cumulative mortality (for the individual clusters) is modeled as a function of time and cluster level covariates. The problem of fitting cumulative mortality as a function of time to clustered data involves modeling the multinomial response over time. An additional aspect here is the possibility of extra-multinomial variation (overdispersion) arising from the use of clusters as the experimental units. A consequence of failing to take overdispersion into account is not only underestimation of the standard errors of estimated regression coefficients but also those of the lethal time $L T_{p}$ - the time until a certain proportion $p$ of insects have died - giving incorrect significance of treatment effects (Hinde and Demétrio, 1998) and confidence intervals that are too short.

For this particular type of data, there are only a few appropriate methods and models that can be applied. Standard approaches include survival analysis 
(Petkau and Sitter, 1989) and ordinal generalized linear models (McCullagh, 1980; Glonek and McCullagh, 1995). However, these approaches are questionable when there is extra-multinomial variability in the data. Whatever the approach taken, it needs to be able to incorporate the possibility of overdispersion arising from the use of clusters as the experimental units.

The use of Liang and Zeger's (1986) generalized estimating equations (GEE) as a multivariate version of quasi-likelihood (QL) (McCullagh and Nelder, 1989) has been considered as an approach to the problem of fitting a generalized linear model (GLM) to clustered data. The GEE approach addresses the problem of correlated or overdispersed data by using an adjusted score equation for the parameter estimates. We have to specify only the first two moments of the response vector for each cluster, assuming a form for the functional relationship between the mean and the variance. Here there is no need to specify the entire likelihood, which is often intractable for non-Gaussian data, even if additional assumptions are made. For Gaussian data the first and second moments fully identify the likelihood, but with non-Gaussian data it is necessary to make additional assumptions about higher-order moments.

In this paper we use the generalized linear model framework for multinomial data to model overdispersion in clustered data following the approach given in O'Hara Hines and Lawless (1993). We use a logit link function and day as an explanatory variable, in all of the models that we present here. The first model we consider is the standard cumulative multinomial model. The second model is an extension to multinomial data of the beta-binomial model, which is often used for overdispersed binomial data. This model compounds the multinomial distribution for the observed counts with a Dirichlet distribution for the vector of underlying probabilities, leading to a Dirichlet-multinomial distribution, which has a different form for the variance function to the standard multinomial distribution. The third model is a random intercept model, where we incorporate an additive random effect in the linear predictor to give a random location shift for each disctinct multinomial 
sample. The final model is an extension of third model and includes both random intercepts and random slopes. 


\section{INTRODUÇÃO}

As pragas de culturas (cupim, ácaro, besouro etc) podem ser consideradas como um dos fatores importantes que limitam a produção agrícola em todo o mundo. $\mathrm{O}$ controle delas através de inimigos naturais (controle biológico) tem se mostrado como uma alternativa útil na minimização de problemas dessa natureza. Segundo Alves (1998), o controle microbiano representa um ramo do controle biológico que trata da utilização racional dos patógenos (fungos, vírus, bactérias e nematóides) no controle de pragas de culturas.

Em termos de Brasil, em particular no Estado de São Paulo, o cupim subterrâneo da espécie Heterotermes tenuis é uma das pragas da cana-de-açúcar que gera prejuízos na produção, da ordem de 10 ton./ha/ano (Novaretti, 1985). Esse inseto infesta a plantação de cana-de-açúcar na época do plantio, matando os brotos em germinação, ou durante a fase de maturação, reduzindo assim o número de plantas novas após a primeira colheita. $\mathrm{O}$ controle dessa praga é dificultado pelo fato de os ninhos de $H$. tenuis serem subterrâneos, necessitando-se de inseticidas de alto poder residual (organo-clorados), os quais acarretam sérios problemas ao meio-ambiente e ao homem. Devido a isso, a aplicação desses inseticidas em cana-de-açúcar foi proibida pelo Ministério da Agricultura em setembro de 1985. Segundo Fernandes e Alves (1991), a aplicação de fungos do gênero Beauveria spp (spp indica várias espécies) apresentou-se como um método de controle alternativo e mostrou-se um candidato em potencial no controle de $H$. tenuis por possuir a característica de viver tanto no hospedeiro (inseto ou ácaro) como também fora dele, ou seja nos ninhos 
(Almeida et al., 1997).

Outra praga de importância econômica é o ácaro rajado Tetranychus urticae que causa grandes prejuízos em lavouras de plantas olerícolas e em plantas ornamentais. Segundo Tamai (1997), durante as décadas de 50 e 60, foram desenvolvidas pesquisas com alguns patógenos de ácaro (fungos e vírus em sua grande maioria), contudo não tiveram continuidade pelas dificuldades de estruturação de um sistema econômico de produção em larga escala. O controle desse artrópodo é feito quase sempre pela aplicação de produtos químicos. Para culturas hortícolas em que a produção é frequentemente consumida de forma in natura, os resíduos dos produtos que permanecem sobre os alimentos representam um sério risco à saúde dos consumidores.

Com o objetivo de estudar a virulência (velocidade de matar) e o grau de patogenicidade (capacidade do microrganismo de provocar doença) do fungo Beauveria spp no cupim $H$. tenuis e no ácaro T. urticae, têm sido realizados experimentos no laboratório de Patologia dos Insetos do Depto. de Entomologia da ESALQ/USP, sob a orientação do Professor Dr. Sérgio Batista Alves. Em um desses experimentos, Almeida (1994) utilizou isolados de fungos da espécie Beauveria bassiana sobre o cupim Heterotermes tenuis e observou o número de insetos mortos diariamente, durante 8 dias após a aplicação do patógeno em 30 insetos. Em um outro experimento, Tamai (1997) utilizou isolados de Beauveria bassiana para o controle do ácaro Tetranychus urticae, observando a mortalidade diária dos insetos, durante 6 dias após a aplicação do patógeno em 10 insetos.

Frequentemente, em bioensaios entomopatogênicos, diferentes condições de tratamento (os isolados, por exemplo) são aplicadas a grupos de insetos ou ácaros e a resposta (contagem ou proporção de mortos) em cada grupo é medida em períodos sucessivos de tempo, que podem ser expressos em horas, dias ou semanas. Isso é semelhante ao que é feito em ensaios de toxicidade, em que são aplicadas diferentes concentrações de uma ou mais drogas a grupos de indivíduos. Essa característica de natureza longitudinal, onde as medidas da variável resposta 
em cada grupo de insetos são observadas em várias ocasiões no tempo, exige uma atenção especial para a dependência entre as medidas realizadas no tempo dentro de uma mesma unidade experimental.

Uma outra característica que deve ser considerada é o uso de grupos de insetos como unidades experimentais. Isso pode acarretar aos dados uma variabilidade maior do que a suposta pelo modelo probabilístico (superdispersão). Como consequência os erros padrões para os efeitos dos fatores ou covariáveis ficam subestimados, causando assim um comprometimento das inferências obtidas.

O desenvolvimento de metodologias estatística para a análise de dados contínuos tem acontecido de uma forma mais rápida do que para dados discretos. Somente nas quatro últimas décadas é que se verificoue um crescimento considerável na metodologia para dados discretos. Em diversas áreas do conhecimento científico (Biologia, Agronomia, Entomologia e Educação, dentre outras) é cada vez mais frequente deparar-se com conjunto de dados que apresentem estruturas mais complexas (resposta politômica, superdispersão e estrutura de agrupamento, dentre outras). De maneira mais específica, dados multivariados discretos que se apresentam na forma agrupada (cluster), onde as respostas possam vir de maneira repetida, podendo assim induzir uma estrutura de correlação.

A problemática da suposição de não normalidade da variável resposta multivariada dificulta o uso da modelagem probabilística clássica através da verossimilhança. Isso deve-se ao fato da escassez de distribuições multivariadas alternativas que apresentem as mesmas propriedades da distribuição normal (Zeger \& Liang, 1986; Artes, 1997). Uma alternativa é o uso das equações de estimação generalizadas (EEG) propostas por Zeger \& Liang (1986) para a obtenção da estimativa dos parâmetros dos modelos de resposta multivariada nos quais, o modelo probabilístico não precisa ser completamente conhecido, bastando apenas a identificação do primeiro e segundo momentos. As EEG de Zeger \& Liang (1986) são uma generalização, para o caso multivariado, da quase-verossimilhança de Wedderburn (1974) e Zeger et al. (1988) mostraram as equivalências entre as EEG e os modelos 
lineares generalizados de McCullagh \& Nelder (1989).

Autores como O'Hara Hines et al. (1992) e Hall (1998) fizeram uso das EEG para a análise de dados agrupados (cluster) e O’Hara Hines \& Lawless (1993) usaram as EEG para a estimação dos parâmetros do modelo de regressão e método dos momentos (McCullagh \& Nelder, 1989) para a estimação do parâmetro de superdispersão (Hinde \& Demétrio, 1998a, b) para dados agrupados (cluster) provenientes de estudos toxicológicos. Seguindo essa metodologia, o presente trabalho tem como objetivos:

(i) modelar dados de patogenicidade e virulência dos isolados de $B$. bassiana para controle do cupim H. tenuis na cultura de cana-de-açúcar e do ácaro T. urticae na cultura de plantas olerícolas e em plantas ornamentais, usando o enfoque de Modelos Lineares Generalizados;

(ii) explorar diferentes parametrizações no processo de modelagem da superdispersão;

(iii) desenvolver os programas computacionais necessários para o uso dos diferentes modelos ;

(v) apresentar considerações sobre as medidas de virulência do patógeno que possam ser aplicadas no campo;

(vi) apresentar considerações sobre o planejamento de experimentos para esse tipo de estudo. 


\section{REVISÃO DE LITERATURA}

Em diferentes áreas de aplicações dos métodos estatísticos, os pesquisadores deparam-se com observações obtidas em escala discreta, na forma de proporções (dados binários) ou contagens. Como exemplos na área de experimentação agronômoca podem ser citados: a germinação, ou não, de sementes expostas a determinadas condições experimentais; um inseto sobreviver ou morrer quando exposto a uma determinada dose de um inseticida; número de esporos de fungos em placa de petri; número de brotos produzidos por explante em experimentos de micropropagação; grau de coloração das folhas de plantas tratadas com adubos químicos.

$\mathrm{O}$ interesse pela modelagem de dados de proporções e contagens data da década de 40 (Finney, 1947). O desenvolvimento constante da metodologia experimental nas diversas áreas do conhecimento científico, particularmente em Entomologia, tem levado à busca de procedimentos estatísticos mais eficientes e precisos para a modelagem de dados discretos. Dados desses tipos são usualmente modelados através das distribuições binomial, Poisson ou multinomial associadas a modelos de regressão, e são casos particulares dos Modelos Lineares Generalizados - MLG (Nelder \& Wedderburn, 1972). Esses procedimentos generalizaram a análise de variância, usada para dados com distribuição Normal, através da análise de deviance (Cordeiro, 1986) como alternativa para dados com distribuições pertencentes à família exponencial na forma canônica (Bickel \& Doksum, 1977), eliminando assim a necessidade de transformação nos dados originais. Essa generalização permitiu a ligação entre a análise de dados discretos e contínuos. Como exemplos podem ser citados os modelos log-lineares para tabelas de contingência, modelos logístico, pro- 
bit e complemento log-log para dados de proporções e os modelos para análise de sobrevivência.

Os Modelos Lineares Generalizados (MLG), desenvolvidos por Nelder \& Wedderburn (1972), são uma extensão dos modelos lineares clássicos de GaussMarkov e são definidos através de três componentes (McCullagh \& Nelder, 1989; Demétrio, 1993):

(i) Componente aleatório: $Y_{1}, Y_{2}, \ldots, Y_{n}$ são variáveis aleatórias independentes, pertencentes à família exponencial, com $E\left(Y_{i}\right)=\mu_{i}$ e um parâmetro de escala, conhecido $\phi>0$. Cada componente $Y_{i}$ tem sua função de densidade de probabilidade (ou função de probabilidade no caso discreto) na forma

$$
f_{Y_{i}}\left(y_{i} ; \theta, \phi\right)=\exp \left\{\frac{1}{a(\phi)}\left[y_{i} \theta_{i}-b\left(\theta_{i}\right)\right]+c\left(y_{i} ; \phi\right)\right\}
$$

onde $a(),. b($.$) e c($.$) são funções conhecidas, sendo que \mu_{i}=E\left(Y_{i}\right)=b^{\prime}\left(\theta_{i}\right)$ e $\operatorname{Var}\left(Y_{i}\right)=a(\phi) b^{\prime \prime}\left(\theta_{i}\right)=a(\phi) \frac{\partial \mu_{i}}{\partial \theta_{i}}=a(\phi) V(\mu) . V(\mu)$ é chamada de função de variância e, em geral, $a(\phi)=\phi / w$, onde $w$ são pesos a priori. Quando $\phi$ é conhecido (1) pertence à família exponencial na forma canônica a um parâmetro e $\theta_{i}$ é chamado de parâmetro canônico ou natural;

(ii) Componente Sistemático: um preditor linear dado por

$$
\eta_{i}=\sum_{j=1}^{q} x_{j i} \beta_{j} \quad \text { ou } \quad \boldsymbol{\eta}=\boldsymbol{X} \boldsymbol{\beta}
$$

onde X é uma matriz, de dimensões $n \times q$, de delineamento (covariáveis ou variáveis dummy para níveis de fatores) e $\boldsymbol{\beta}$ é um vetor, de dimensões $q \times 1$, de parâmetros a serem estimados $(q \leq n)$;

(iii) Função de Ligação: uma função monótona diferenciável que liga o componente aleatório ao componente sistemático:

$$
\eta_{i}=g\left(\mu_{i}\right)
$$

Quando $g\left(\mu_{i}\right)=\theta_{i}$ o preditor linear $\left(\eta_{i}\right)$ está modelando o parâmetro canônico $\left(\theta_{i}\right)$, isto é, $\eta_{i}=\theta_{i}$, e a função de ligação é chamada de função de ligação 
canônica ou natural. Os estimadores de máxima verossimilhança para os parâmetros do modelo, $\hat{\boldsymbol{\beta}}$, são obtidos usando-se o método escore de Fisher, implementado através do algoritmo de mínimos quadrados reponderados iterativamente (McCullagh \& Nelder, 1989). Dentre as funções de ligação mais comuns na modelagem de dados de proporções estão logit, probit e complemento log-log e para dados de contagens, a ligação logarítmica.

Como uma primeira referência em português, Cordeiro (1986) descreve detalhadamente os procedimentos de estimação nos MLG e Demétrio (1993) apresenta os MLG com aplicações na área de agronomia, ilustrando vários modelos que podem ser usados para dados com distribuições de Poisson e binomial.

Sendo a principal referência em MLG, McCullagh \& Nelder (1989) fazem um apanhado completo dos modelos, métodos de estimação e análise de diagnóstico para dados contínuos, de contagens e proporções. No capítulo para dados de resposta multinomial, denominadas de respostas politômicas, é feita uma excelente descrição das escalas de medida (nominal, ordinal ou intervalar), ressaltando a necessidade e a importância da distinção entre elas. Apresentam um apanhado completo sobre a distribuição multinomial, e o uso da mesma na modelagem de dados de resposta multinomial através dos modelos logístico e complemento log-log, para dados categorizados ordinais, e os modelos log-lineares, para dados com escala de medidas nominal e intervalar. Mostram as similaridades entre os diferentes modelos, como também suas particularidades. Os procedimentos utilizados na estimação e no ajuste dos MLG utilizados no pacote computacional GLIM (Payne, 1986) foram descritos por Aitkin et al. (1989).

De forma mais reduzida, Dobson (1990) apresenta uma introdução aos MLG enfatizando as aplicações e o uso dos modelos de regressão múltipla e ANOVA para dados contínuos, regressão logística para dados binários e modelos log-lineares para tabelas de contingência bidimensionais. Uma descrição mais ampla e detalhada dos modelos para dados binários e binomiais é apresentada em Collet (1991), que também discute questões como dose efetiva $\left(L D_{50}\right)$, potência relativa e resposta 
natural, temas relevantes nos ensaios de dose-resposta.

As aplicações de modelos lineares logísticos para a análise de dados binomiais e os modelos log-lineares para contagens e dados multinomiais, no enfoque de MLG desenvolvidos por Nelder \& Wedderburn (1972), acomodam uma determinada estrutura para a variabilidade da variável resposta. Grande parte dos dados provenientes de estudos teratológicos, farmacológicos e entomológicos apresentam uma variabilidade observada superior àquela esperada, ou suposta, pelo modelo adotado. A essa possível fonte de variabilidade extra, não contemplada pela suposição dos modelos, dá-se o nome de superdispersão. Na situação inversa, quando a variabilidade observada é menor do que a esperada pelo modelo, tem-se a subdispersão. Na prática é mais comum a existência da superdispersão.

A problemática dos modelos usados em dados que apresentam superdispersão não é recente. Em meados da década de 40, Cochran (1943) teve problemas com variabilidade extra-binomial na modelagem de dados de proporção, com denominadores diferentes e Finney (1947) deparou-se com fonte extra de variabilidade não acomodada pelos modelos para dados de ensaios de dose-resposta.

Na prática, o fenômeno superdispersão não é tão incomum quanto se pensa. McCullagh \& Nelder (1989) afirmam que, de fato, na prática, a superdispersão é a norma e a dispersão nominal (padrão do modelo) é a exceção. O que diferencia é a intensidade, ou grau de atuação, dessa característica nas diferentes áreas de aplicação. Nas últimas décadas, muitos estudos têm sido desenvolvidos com o uso de MLG para dados superdispersos de contagens e proporções.

Equivalente ao procedimento para comparação de modelos na ANOVA, Nelder \& Wedderburn (1972) propuseram uma medida de discrepância denominada deviance, dada por

$$
d\left(\hat{\boldsymbol{\mu}}_{\boldsymbol{i}}, \boldsymbol{y}_{i}\right)=2\left[l\left(\hat{\boldsymbol{\mu}}_{i}, \boldsymbol{y}_{i}\right)-l\left(\boldsymbol{y}_{i}, \boldsymbol{y}_{i}\right)\right]
$$

em que $l\left(\hat{\boldsymbol{\mu}}_{\boldsymbol{i}}, \boldsymbol{y}_{\boldsymbol{i}}\right)$ é o máximo do logaritmo da função de verossimilhança para o modelo ajustado (corrente) e $l\left(\boldsymbol{y}_{\boldsymbol{i}}, \boldsymbol{y}_{\boldsymbol{i}}\right)$ é o máximo do logaritmo da função de verossimilhança para o modelo saturado. É uma medida utilizada para avaliar a falta de ajuste do 
modelo. Valores grandes da deviance indicam que o modelo não está bem ajustado. Quando o modelo se ajusta bem aos dados a distribuição da deviance converge para uma distribuição $\chi_{n-q}^{2}$ (Qui-quadrado com $n-q$ graus de liberdade), para valores grandes de $n$, no caso de dados discretos. Considerando-se que no caso de uma variável aleatória com distribuição $\chi_{g . l}^{2}$, o valor esperado desta variável é igual ao número de graus de liberdade, isto é, para um modelo bem ajustado espera-se que

\section{Deviance Residual $\simeq$ graus de liberdade.}

Caso isto não aconteça existem fontes extras de variabilidade, não contempladas pelos modelos, interferindo na qualidade do ajuste. Algumas circunstâncias ou fatores que podem acarretar essa falta de ajuste foram descritas por Hinde \& Demétrio (1998a, b) como sendo:

(i) o componente sistemático do modelo $(X \beta)$ é inadequado, isto é, alguma variável explanatória ou combinações delas foram omitidas do preditor linear $(\eta)$;

(ii) a escolha inadequada da função de ligação $g(\mu)$;

(iii) presença de outliers nos dados;

(iv) fonte natural de variabilidade que o componente aleatório (Y) não consegue incorporar ao modelo, fazendo com que a variância nominal seja maior que a esperada.

Para dados com distribuição binomial, Poisson ou multinomial tem-se:

- dados de proporção: $\operatorname{Var}\left(Y_{i}\right)>m_{i} \pi_{i}\left(1-\pi_{i}\right)$,

- dados de contagem: $\operatorname{Var}\left(Y_{i}\right)>\mu_{i}$,

- dados multinomiais: $\operatorname{Var}\left(\boldsymbol{Y}_{i}\right) \neq \boldsymbol{V}_{i}$.

Para as situações $(i),(i i)$ e (iii) citadas anteriormente, essa superdispersão aparente pode ser contornada com o uso de técnicas de diagnóstico descritas em McCullagh \& Nelder (1989) e Collet (1991). No quarto caso, onde existe uma 
fonte natural de variabilidade extra, as possíveis causas dessa superdispersão são descritas por Hinde \& Demétrio (1998b) como sendo:

- uma variabilidade individual natural das unidades experimentais que produzem um componente adicional na variância e que não são quantificadas no modelo pressuposto;

- correlação entre as respostas individuais;

- dados agregados;

- variáveis não observáveis.

A não acomodação dessa superdispersão leva a um resultado de deviance bem maior do que seus graus de liberdade. Desse modo, como consequência, as estimativas dos erros padrões dos $\hat{\beta}^{\prime} s$ ficam subestimadas, causando assim um comprometimento das inferências do modelo. Hinde \& Demétrio (1998a, b) também descreveram alguns mecanismos que podem se usados para acomodação da superdispersão em dados de contagens e proporções superdispersos através das estratégias:

- assumir uma forma genérica para a função de variância $V\left(\boldsymbol{Y}_{i}\right)$, incorporando parâmetros adicionais para quantificar a superdispersão através dos modelos de média-variância;

- assumir um modelo em dois estágios para a variável resposta, ou seja, associando uma distribuição ao parâmetro de média do modelo básico (Beta-binomial e binomial-Negativo) ou ao preditor linear (logístico-Normal e Poisson-Normal) (Hinde \& Demétrio, 1996).

Também apresentaram uma revisão dos modelos de superdispersão para dados de proporçōes e contagens, abordando diferentes métodos de estimação e mostrando as equivalências dos métodos para os modelos adotados. 
Como uma alternativa ao algoritmo de estimação do modelo de regressão logística para dados binários do GLIM, Williams (1982) propôs uma modificação que permitiu a acomodação da superdispersão para dados de proporção. Ele utilizou, de forma iterativa, o algoritmo IRLS (Nelder \& Wedderburn, 1972) para obter as estimativas dos parâmetros do modelo, $\boldsymbol{\beta}$, e o método de quase verossimilhança - QL - (Wedderburn, 1974) para estimar o parâmetro de superdispersão, $\phi$. A cada passo da estimação de $\boldsymbol{\beta}$, o fator $1 /\left[1+\hat{\phi}\left(n_{i}-1\right)\right]$ foi usado como peso. $\mathrm{O}$ cálculo de $\hat{\phi}$ deu-se de maneira iterativa, usando-se $X^{2}=E\left(X^{2}\right)$, onde $X^{2}$ é a estatística generalizada de Pearson (McCullagh \& Nelder, 1989). A mesma filosofia foi utilizada por Breslow (1984) para implementar, no GLIM, uma macro para ajustar modelos log-lineares para dados com variação extra-Poisson. Moore (1987) também usou a mesma técnica para a modelagem da superdispersão em dados com distribuição binomial para o modelo Beta-binomial, Hinde (1996) implementou duas macros no GLIM, que permitiram a modelagem da superdispersão para dados com distribuições de Poisson e binomial com o uso de outras funções de ligação, além da canônica e Hinde (1982) utilizou modelos de superdispersão para dados com distribuição de Poisson.

Livros como Morgan (1992, 1996) e Collet (1991) discutem de forma mais ampla uma variedade de experimentos, com resposta binária e de contagem, que podem apresentar característica de superdispersão, com um grande enfoque na análise de dados provenientes de ensaios do tipo dose-resposta, bastante comuns em entomologia.

Como um critério gráfico para a avaliação da qualidade de ajuste dos modelos, que também pode ser usado para modelos com dados superdispersos, Demétrio \& Hinde (1997) propuseram, desenvolveram e implementaram no GLIM, a utilização dos Half-Normal plots com envelopes de simulação.

Como uma extensão desses modelos, Vieira (1998) trabalhou com modelos de mistura para dados binomiais inflacionados de zeros com superdispersão com dados provenientes de bioensaios de controle biológico e Demétrio (1994) fez uso de 
modelos de superdispersão para análise de dados de produção em cana-de-açúcar.

Em experimentos em que a resposta (proporções ou contagens) está expressa na forma agrupada e é medida em períodos sucessivos de tempo, faz-se necessário o uso de modelos que incorporem essa característica longitudinal. Devido a essa característica eles são conhecidos como bioensaios multivariados de doseresposta.

Muitos autores têm utilizado os modelos de análise de sobrevivência para a análise de dados agrupados. Como exemplos podem ser citados Prentice \& Gloeckler (1978), que ajustaram o modelo de Cox para dados de câncer, agrupados em intervalos (mensais) de tempo, Pierce et al. (1979), que aplicaram os métodos de análise de sobrevivência para dados agrupados provenientes de um ensaio toxicológico para peixes e Petkau \& Sitter (1989) que utilizaram as técnicas de análise de sobrevivência para reanalisar um conjunto de dados agrupados de um ensaio de dose-resposta na área de Zoologia. Esse conjunto de dados foi analisado inicialmente por Carter \& Hubert (1984), através de um modelo multivariado de curva de crescimento. Posteriormente, Petkau \& Sitter (1989) apresentaram críticas severas ao artigo de Carter \& Hubert com relação às suposições de normalidade e independência das observações. Neste artigo já ressaltaram a necessidade do uso de modelos que pudessem levar em consideração a variabilidade entre os diferentes níveis de doses e a variabilidade existente entre os peixes de um mesmo tanque. Chamaram a atenção para o uso dos MLG e dos métodos de quase verossimilhança de Wedderburn (1974), como uma alternativa na análise de dados com essas estruturas. Sugerindo o uso da distribuição Dirichlet-multinomial (Mosimann, 1962) como uma generalização da multinomial para permitir a modelagem de uma possível variação extra-multinomial.

Uma outra abordagem na análise de proporções acumuladas com essa característica longitudinal, pode ser feita através dos modelos categorizados ordinais. Nesse caso, os tempos de 1 a $D$ são considerados como a variável resposta ordinal (existe uma hierarquia no tempo) e os fatores de 1 a $K$ como variável explicativa nominal. 
Em tabelas de contingência de dimensões $K \times D$, em que a resposta é ordinal e a variável explicativa é nominal, os dados são observados na forma de contagens ou proporções das caselas. Nessa situação, a análise é semelhante a uma análise de variância, onde o fator (isolado) possui $K$ níveis. Essa natureza multinomial na resposta é modelada através dos modelos log-lineares e modelos logísticos.

Uma ampla discussão sobre modelos de regressão para dados ordinais é feita por McCullagh (1980) e Agresti $(1990,1996)$. Descrevem os diferentes modelos utilizados para diversos tipos de variáveis resposta - nominal, ordinal e intervalar - e variáveis preditoras, estimação dos parâmetros dos modelos e as equivalências entre os mesmos. Esses modelos também foram utilizados por Green (1984) e Glonek (1996) para a modelagem de dados longitudinais, considerando o tempo como uma variável latente e Wolfe (1996) implementou no GLIM, uma macro para o ajuste de modelos com resposta ordinal utilizando o modelo logit cumulativo e um modelo de logit cumulativo com efeito de escala, que permitiram uma mudança na forma da distribuição da variável latente (tempo). McCullagh (1980), recomendou que o uso desses modelos somente seja feito quando o número de respostas categorizadas for superior ou igual a três.

O uso das EEG de Zeger \& Liang (1986) para análise longitudinal de dados discretos e contínuos abriu um leque de opções para o ajuste de MLG com dados de natureza multivariada. Autores como Zeger et al. (1988), Pendergast et al. (1996) e Albert (1999) mostraram as equivalências entre as EEG e os modelos lineares generalizados de McCullagh \& Nelder (1989). O'Hara Hines et al. (1992) e Hall (1998) fizeram uso das EEG para a análise de dados agrupados (cluster) e O'Hara Hines \& Lawless (1993) usaram as EEG para a estimação dos parâmetros do modelo de regressão e método dos momentos (McCullagh \& Nelder, 1989) para a estimação do parâmetro de superdispersão (Hinde \& Demétrio, 1998a, b) para dados agrupados (cluster) provenientes de estudos toxicológicos. Isso permitiu assim o uso da distribuição multinomial associada a modelos de regressão, modelos de médiavariância e modelos em dois estágios que pudessem acomodar a característica de 
superdispersão e a estrutura de dependência das respostas. De forma mais recente, Betensky \& Williams (2001) compararam modelos para dados binários agrupados, provenientes de estudos clínicos.

Uma abordagem utilizada para a análise de dados de proporções acumuladas no tempo é a classe de modelos não-lineares. Segundo Vonesh \& Chinchilli (1997), os MLG desenvolvidos por Nelder \& Wedderburn (1972) podem ser considerados como uma classe univariada dos modelos não-lineares. Nessa filosofia, a resposta média, $E(Y)$, depende de um conjunto de $m$ covariáveis, $X$, através de uma combinação linear, $E(Y)=\mu=f(X \beta)=f(\eta)$, sendo $g(\mu)=f^{-1}(X \beta)$ uma função monótona inversível, e $\operatorname{Var}(Y)=\sigma^{2} h(\mu)$, sendo $h($.$) a função de variância.$

Muitos autores têm estendido esses métodos para o caso de medidas. Como exemplos podem ser citados Stiratelli et al. (1984), Liang \& Zeger (1986), Zeger et al. (1988) e Breslow \& Clayton (1993), dentre outros. Esses modelos permitem que na matriz de variâncias e covariâncias sejam incorporadas duas fontes de variabilidade: entre indivíduos e entre as observações realizadas em um mesmo indivíduo. Os métodos de estimação são aproximados através dos métodos de máxima verossimilhança, quase verossimilhança e mínimos quadrados reponderados iterativamente.

Os modelos mecanicistas (Morgan, 1992) compõem uma outra classe de modelos que podem ser usados para descrever dados de contagens e proporções com respostas no tempo. Esses modelos caracterizam-se por descreverem os aspectos mecânicos, que apresentam-se de uma forma latente, inerentes ao processo de estudo. Como exemplos, citam-se as caraterísticas de suscetibilidade do hospedeiro e as informações sobre o volume de material consumido por esses hospedeiros (intake), em bioensaios de dose-resposta.

Na realidade, estes modelos têm a estrutura dos modelos não-lineares, em que a variável resposta $(Y)$ é modelada através de uma função não-linear $(f)$ de covariáveis e/ou fatores $(X)$, ou seja, $Y=f(X \beta)$ (Lindsey, 1993). São classificados como mecanicistas por tentarem descrever todos os estágios do processo em estudo. 
Têm, em geral, a grande vantagem de permitir, através dos parâmetros do modelo, uma melhor interpretação das etapas inerentes no processo em estudo (infecção, micropropagação, superparasitismo e infestação, dentre outros).

Vários exemplos de dados que são analisados usando-se modelos que podem ser classificados como mecanicistas estão descritos em Morgan (1992). Dentre outros, citam-se Puri (1975), Hewlett (1974), Hartley \& Sielken (1977) e Ridout et al. (1993). Terrill et al. (1998) compararam os resultados produzidos pelo uso dos modelos categorizados ordinais (McCullagh, 1980) e um outro modelo mecanicista para dados de dose-resposta de bioensaios de controle biológico do besouro Otiorhynchus sulcatus em videiras.

Os modelos que estão descritos na seção 4 e que serão utilizados neste trabalho, são considerados por Morgan (1992) como modelos descritivos ou analíticos. Morgan (1992) comenta que, em algumas situaçōes, os modelos mecanicistas reduzem-se aos modelos descritivos. 


\section{MATERIAL}

Os dados utilizados neste trabalho são provenientes de dois bioensaios toxicológicos para controle microbiano do cupim H. tenuis e do ácaro T. urticae, realizados no Laboratório de Patologia dos Insetos do Departamento de Entomologia da ESALQ/USP, desenvolvidos por Almeida (1994) para o controle do cupim e por Tamai (1997) para o controle do ácaro, ambos sob a orientação do Prof. Dr. Sérgio Batista Alves.

Para os dois bioensaios, experimentos preliminares foram realizados para definir a concentração dos isolados $\left(5 \times 10^{8}\right.$ conidia $\left./ \mathrm{ml}\right)$ e o método de inoculação dos cupins (pulverização com torre de Poter, ver Almeida, 1994) e dos ácaros (imersão dos discos de folhas, ver Tamai, 1997).

(i) Estudo do fungo Beauveria bassiana para o controle microbiano da praga do cupim Heterotermes tenuis:

O cupim subterrâneo da espécie Heterotermes tenuis é uma das pragas da cana-de-açúcar que gera prejuízos na produção da ordem de 10 ton./ha/ano (Novaretti, 1985). O controle dessa praga é dificultado pelo fato de os ninhos do $H$. tenuis serem subterrâneos, necessitando-se de inseticidas de alto poder residual (organo-clorados), os quais acarretam sérios problemas ao meio-ambiente e ao homem. Devido a isso, a aplicação desses inseticidas em cana-de-açúcar foi proibida pelo Ministério da Agricultura em setembro de 1985. Segundo Fernandes \& Alves (1991), a aplicação de fungos do gênero Beauveria spp apresentou-se como um método de controle alternativo e mostrou-se um candidato em potencial no controle do $H$. 
tenuis por possuir a característica de viver tanto no hospedeiro como também fora dele, ou seja nos ninhos (Almeida et al., 1997).

Neste experimento, 5 placas de criação, com 30 cupins por placa, foram pulverizadas com os isolados (patógenos) de fungo. Cada placa foi considerada como sendo uma repetição. Os cupins mortos foram contados diariamente, durante um período de 8 dias. Neste ensaio foram utilizados 142 isolados de B. bassiana. Os dados de mortalidade dos cupins para dois isolados estão apresentados na Tabela 1. Nas Figuras 1 a 8 (páginas 50 a 57) estão apresentados os diagramas de dispersão para as proporções de morte acumulada do cupim $H$. tenuis para os isolados de $B$. bassiana utilizados nos experimentos.

(ii) Estudo do fungo Beauveria bassiana para o controle microbiano do ácaro Tetranychus urticae:

O ácaro rajado T. urticae Koch ataca muitas culturas de importância econômica. É considerada uma das pragas mais importantes das plantas olerícolas e ornamentais em todo mundo.

O controle deste artrópodo é feito quase sempre pela aplicação de produtos químicos. Para culturas hortícolas em que a produção é frequentemente consumida na forma in natura, os resíduos dos produtos que permanecem sobre os alimentos representam um sério risco à saúde dos consumidores. Por outro lado, aplicações feitas de forma preventiva ou mesmo quando a praga ainda se encontra em infestações ainda pequenas, têm favorecido o surgimento de populações resistentes deste ácaro.

Neste experimento, 12 discos (arenas) de folhas de feijão-de-porco Canavalia ensiformis foram inoculados (por imersão) com isolados de B. bassiana (patógenos) e colocados em placas de Petri. Sobre cada disco foram colocados 10 ácaros e o número de mortos foi contado diariamente, durante um período de 6 dias. Isso foi feito para 80 isolados de $B$. bassiana. Os dados de mortalidade dos ácaros para dois isolados estão apresentados na Tabela 2. Nas Figuras 11 a 16 (páginas 
11 a 16) estão apresentados os diagramas de dispersão para as proporções de morte acumulada do ácaro T. urticae para os isolados de $B$. bassiana utilizados nos experimentos.

Em uma análise visual dos dados de mortalidade para os dois ensaios, apresentados nas Figuras 1 a 8 e nas Figuras 11 a 16, observa-se que existe uma variabilidade considerável dos interceptos e dos coeficientes angulares entre as repetições para muitos dos isolados nos dois bioensaios. Isso indica uma necessidade em considerarem-se modelos com efeitos aleatórios no intercepto e no coeficiente angular da regressão.

Verifica-se que, para alguns dos isolados, existe um alto grau de virulência, ou seja, a mortalidade inicial dos insetos é grande e rápida e se mantém crescente ao longo do experimento. Para outros isolados o grau de virulência é mais fraco, isto é, mesmo próximo do final do experimento a mortalidade apresentada é muito baixa.

Para ensaios dos tipos apresentados em (i) e (ii) a interpretação dos parâmetros do modelo de regressão da mortalidade em função dos isolados e do tempo é de fundamental importância para os entomologistas. Desse modo é possível identificar o(s) isolado(s) com um alto poder de virulência, capacidade de matar mais rápido (velocidade de matar), possibilitando a seleção dos isolados que tenham alta patogenicidade. Tais informações podem ser obtidas através dos coeficientes angulares das retas para os diferentes isolados, pois indicam o grau de agressividade dos isolados - considerando-se retas com interceptos bem próximos, a reta com um coeficiente angular maior identifica o isolado mais agressivo. Por outro lado, considerando-se as retas com coeficientes angulares bem próximos, a reta que apresentar o intercepto maior identificará o isolado mais agressivo. Essas informações são refletidas diretamente no tempo letal, $L T_{p}$, tempo que o isolado leva para matar $p \%$ dos insetos. Essa medida também pode ser usada para comparar a virulência de isolados que causam a mesma mortalidade, identificando assim os isolados que matam mais rapidamente os insetos. 
Com essas informações a priori, obtidas em ensaios laboratoriais, os pesquisadores têm respaldo para traçar as diretrizes dos ensaios de campo. Desse modo, o controle biológico no campo já pode ser iniciado com os patógenos das melhores linhagens. 
Tabela 1: Mortalidade acumulada de cupins após oito dias de exposição a dois isolados de B. bassiana.

\begin{tabular}{c|c|cccccccc}
\hline Isolados & Réplica & \multicolumn{7}{|c}{ dias } \\
\hline \multirow{5}{*}{848} & & 1 & 2 & 3 & 4 & 5 & 6 & 7 & 8 \\
\hline \multirow{7}{*}{787} & 2 & 0 & 4 & 6 & 9 & 11 & 13 & 13 & 16 \\
& 3 & 0 & 9 & 9 & 12 & 15 & 20 & 20 & 20 \\
& 4 & 0 & 8 & 12 & 12 & 22 & 24 & 24 & 24 \\
& 5 & 0 & 9 & 12 & 18 & 18 & 18 & 18 & 18 \\
\hline \multirow{7}{*}{} & 1 & 0 & 0 & 0 & 11 & 13 & 15 & 21 & 23 \\
& 2 & 0 & 0 & 0 & 11 & 19 & 20 & 22 & 24 \\
& 3 & 0 & 0 & 2 & 5 & 13 & 13 & 13 & 14 \\
& 4 & 0 & 0 & 0 & 4 & 10 & 10 & 12 & 14 \\
& 5 & 0 & 0 & 10 & 21 & 25 & 30 & 30 & 30 \\
\hline
\end{tabular}

Tabela 2: Mortalidade acumulada de ácaros após oito dias de exposição a dois isolados de B. bassiana.

\begin{tabular}{c|c|cccccc|c|c|cccccc}
\hline Isolado & Arena & \multicolumn{6}{|c|}{ dias } & Isolado & Arena & \multicolumn{7}{|c}{ dias } \\
& & 1 & 2 & 3 & 4 & 5 & 6 & & & 1 & 2 & 3 & 4 & 5 & 6 \\
\hline \multirow{5}{*}{307} & 1 & 0 & 4 & 6 & 9 & 10 & 10 & & 1 & 0 & 0 & 0 & 1 & 1 & 3 \\
& 2 & 0 & 6 & 6 & 8 & 10 & 10 & & 2 & 0 & 0 & 1 & 1 & 4 & 4 \\
& 3 & 0 & 1 & 3 & 9 & 10 & 10 & & 3 & 0 & 0 & 0 & 2 & 5 & 3 \\
& 4 & 0 & 3 & 3 & 7 & 10 & 10 & & 4 & 0 & 0 & 0 & 0 & 0 & 0 \\
& 5 & 0 & 4 & 6 & 8 & 10 & 10 & & 5 & 0 & 0 & 0 & 0 & 2 & 2 \\
& 6 & 0 & 6 & 7 & 9 & 10 & 10 & 485 & 6 & 0 & 0 & 0 & 0 & 2 & 2 \\
& 7 & 0 & 6 & 7 & 8 & 10 & 10 & & 7 & 0 & 0 & 0 & 0 & 0 & 1 \\
& 8 & 0 & 6 & 8 & 9 & 10 & 10 & & 8 & 0 & 0 & 0 & 0 & 2 & 2 \\
& 9 & 0 & 4 & 6 & 7 & 10 & 10 & & 9 & 0 & 0 & 1 & 2 & 3 & 4 \\
& 10 & 0 & 4 & 5 & 9 & 10 & 10 & & 10 & 0 & 0 & 0 & 1 & 2 & 2 \\
& 11 & 0 & 3 & 6 & 6 & 7 & 10 & & 11 & 0 & 0 & 0 & 0 & 0 & 0 \\
& 12 & 0 & 3 & 5 & 7 & 7 & 10 & & 12 & 0 & 0 & 0 & 0 & 1 & 3 \\
\hline
\end{tabular}




\section{MÉTODOS}

Utilizando-se as proporções de mortalidade acumulada obtidas dos ensaios descritos na Seção 3, os diferentes isolados serão considerados como fatores $(i=1,2, \ldots, I ; I=142$ ou 80$)$ e o tempo (dias) como variável explicativa $(j=1,2, \ldots, D ; D=8$ ou 6$)$.

Seja $y_{i k, j}$ o número observado de insetos mortos no tempo $j$ dos $n_{i k}$ insetos que foram expostos ao isolado $i$, na repetição $k$ (Tabela 3). Seja $r_{i k, j}$ a proporção acumulada observada de insetos mortos até o tempo $j$ e que foram expostos ao isolado $i$, na repetição $k$ (Tabela 4 ).

Tabela 3: Números $\left(y_{i k, j}\right)$ observados de insetos mortos no tempo $j$, para o isolado $i$ nas diferentes repetições.

\begin{tabular}{c|c|cccccc|c}
\hline Isolado & Repetição & \multicolumn{5}{|c|}{ Tempo } & Totais \\
& & 1 & $\ldots$ & $j$ & $\ldots$ & $D$ & \\
\hline \multirow{6}{*}{$i$} & 1 & $y_{i 1,1}$ & $\ldots$ & $y_{i 1, j}$ & $\ldots$ & $y_{i 1, D}$ & $n_{i 1}$ \\
& $\ldots$ & $\ldots$ & $\ldots$ & $\ldots$ & $\ldots$ & $\ldots$ & $\ldots$ \\
& $k$ & $y_{i k, 1}$ & $\ldots$ & $y_{i k, j}$ & $\ldots$ & $y_{i k, D}$ & $n_{i k}$ \\
& $\ldots$ & $\ldots$ & $\ldots$ & $\ldots$ & $\ldots$ & $\ldots$ & $\ldots$ \\
& $\mathrm{K}$ & $y_{i K, 1}$ & $\ldots$ & $y_{i K, j}$ & $\ldots$ & $y_{i K, D}$ & $n_{i K}$ \\
\hline
\end{tabular}


Tabela 4: Proporções $\left(r_{i k, j}\right)$ acumuladas observadas de insetos mortos até o tempo $j$, para o isolado $i$ nas diferentes repetições.

\begin{tabular}{c|c|ccccc}
\hline Isolado & Repetição & \multicolumn{5}{|c}{ Tempo } \\
& & 1 & $\ldots$ & $j$ & $\ldots$ & $D$ \\
\hline \multirow{1}{*}{$i$} & 1 & $r_{i 1,1}$ & $\ldots$ & $r_{i 1, j}$ & $\ldots$ & $r_{i 1, m}$ \\
& $\ldots$ & $\ldots$ & $\ldots$ & $\ldots$ & $\ldots$ & $\ldots$ \\
& $\ldots$ & $r_{i k, 1}$ & $\ldots$ & $r_{i k, j}$ & $\ldots$ & $r_{i k, m}$ \\
& $\ldots$ & $\ldots$ & $\ldots$ & $\ldots$ & $\ldots$ \\
\hline
\end{tabular}

Seguindo-se a metodologia usada por O'Hara Hines \& Lawless (1993), os modelos que serão utilizados neste trabalho, para a modelagem dos dados descritos na Seção 3, são relatados a seguir.

\subsection{Modelo multinomial}

Considere que na Tabela 3 a $i$-ésima linha corresponde ao vetor de observações de um vetor aleatório multinomial, $\boldsymbol{Y}_{i k}=\left(Y_{i k, 1}, \ldots, Y_{i k, D}\right)^{T}$, em que $\sum_{j=1}^{D} y_{i k, j}=n_{i k}$ e $\pi_{i k}=\left(\pi_{i k, 1}, \ldots, \pi_{i k, D}\right)^{T}$, sendo $\pi_{i k, j}$ a probabilidade de um inseto exposto ao $i$-ésimo isolado, na repetição $k$, morrer no dia $j$. Então, a distribuição de probabilidade do vetor aleatório $\boldsymbol{Y}_{i k}$ é dada por:

$$
P\left(\boldsymbol{Y}_{i k}=\boldsymbol{y}_{i k} ; n_{i k}, \pi_{i k}\right)=\frac{n_{i k} !}{\prod_{j=1}^{D} y_{i k, j} !} \pi_{i k, 1}^{y_{i k, 1}} \ldots \pi_{i k, D}^{y_{i k, D}}
$$

$\operatorname{com} 0 \leq y_{i k, j} \leq n_{i k}, i=1, \ldots, I, j=1, \ldots, D, k=1, \ldots, K$ e $\sum_{j=1}^{D} \pi_{i k, j}=1$. Tem-se que (2) define a distribuição multinomial com vetor de médias

$$
E\left(Y_{i k}\right)=n_{i k} \pi_{i k}=n_{i k}\left(\pi_{i k, 1}, \ldots, \pi_{i k, D}\right)^{T}
$$

e matriz de variâncias e covariâncias, simétrica, de dimensões $D \times D$, dada por: 


$$
\operatorname{Var}\left[\boldsymbol{Y}_{i k}\right]=\boldsymbol{\Sigma}_{i k}=n_{i k}\left[\operatorname{diag}\left\{\boldsymbol{\pi}_{i k}\right\}-\boldsymbol{\pi}_{i k} \boldsymbol{\pi}_{i k}^{T}\right] .
$$

Uma matriz inversa generalizada para (3) pode ser dada, por exemplo, por

$$
\Sigma_{i k}^{-}=\frac{1}{n_{i k}} \operatorname{diag}\left\{1 / \pi_{i k, 1}, 1 / \pi_{i k, 2}, \ldots, 1 / \pi_{i k, D}\right\} .
$$

Conjuntamente, as $I K$ amostras aleatórias de resposta multinomial, $\boldsymbol{Y}=\left(\boldsymbol{Y}_{11}, \ldots, \boldsymbol{Y}_{I K}\right)^{T}$, têm distribuição de probabilidade definida por

$$
P(\boldsymbol{Y}=\boldsymbol{y} ; \boldsymbol{n}, \boldsymbol{\pi})=\prod_{i=1}^{I} \prod_{k=1}^{K} P\left(\boldsymbol{Y}_{i k}=\boldsymbol{y}_{i k} ; n_{i k}, \boldsymbol{\pi}_{i k}\right)=\prod_{i=1}^{I} \prod_{k=1}^{K} n_{i k} ! \prod_{j=1}^{D} \frac{\pi_{i k, j}^{y_{i k, j}}}{y_{i k, j} !},
$$

em que $\boldsymbol{n}=\left(n_{11}, \ldots, n_{1 K}\right)^{T}$ e $\pi=\left(\pi_{11}, \ldots, \pi_{1 K}\right)^{T}$, com vetor de médias e matriz de variâncias e covariâncias dadas, respectivamente, por

$$
E(\boldsymbol{Y})=\left(E\left(\boldsymbol{Y}_{11}\right), \ldots, E\left(\boldsymbol{Y}_{I K}\right)\right)^{T}=\left(n_{11} \boldsymbol{\pi}_{11}, \ldots, n_{I K} \boldsymbol{\pi}_{I K}\right)^{T}
$$

e

$$
\boldsymbol{\Sigma}=\operatorname{diag}\left\{\Sigma_{11}, \ldots, \boldsymbol{\Sigma}_{I K}\right\}=\left[\begin{array}{ccccc}
\Sigma_{11} & 0 & \ldots & \ldots & 0 \\
0 & \ldots & \ldots & \ldots & 0 \\
\ldots & \ldots & \Sigma_{i k} & \ldots & \ldots \\
\ldots & \ldots & \ldots & \ldots & 0 \\
0 & \ldots & \ldots & 0 & \Sigma_{I K}
\end{array}\right]
$$

uma matriz bloco diagonal, de dimensões $I K D \times I K D$.

$\mathrm{O}$ logaritmo neperiano da função de verossimilhança para as $I K$ observações multinomiais é dado por

$$
l(\boldsymbol{\pi}, \boldsymbol{y})=\ln P(\boldsymbol{Y}=\boldsymbol{y} ; \boldsymbol{n}, \boldsymbol{\pi})=\sum_{i=1}^{I} \sum_{k=1}^{K} \sum_{j=1}^{D} y_{i k, j} \ln \left(\pi_{i k, j}\right)+\sum_{i=1}^{I} \sum_{k=1}^{K} \sum_{j=1}^{D} \ln \left(n_{i k} ! / y_{i k, j} !\right),
$$

sujeito à restrição de que $\sum_{j=1}^{D} \pi_{i k, j}=1$. A derivada parcial de (5) em relação a cada $\pi_{i k, j}$ fica

$$
\frac{\partial l(\boldsymbol{\pi}, \boldsymbol{y})}{\partial \pi_{i k, j}}=\frac{y_{i k, j}-n_{i k} \pi_{i k, j}}{\pi_{i k, j}}=\pi_{i k, j}^{-1}\left(y_{i k, j}-n_{i k} \pi_{i k, j}\right),
$$

de modo que, para a $i k$-ésima observação multinomial e usando-se (4), o sistema de equações de verossimilhança é descrito por 


$$
\frac{\partial l(\boldsymbol{\pi}, \boldsymbol{y})}{\partial \boldsymbol{\pi}_{i k}}=n_{i k} \boldsymbol{\Sigma}_{i k}^{-}\left(\boldsymbol{y}_{i k}-n_{i k} \boldsymbol{\pi}_{i k}\right) .
$$

O estimador de máxima verossimilhança para $\pi_{i k}$ é dado pela solução do sistema (6), obtendo-se

$$
n_{i k} \boldsymbol{\Sigma}_{i k}^{-}\left(\boldsymbol{y}_{i k}-n_{i k} \hat{\pi}_{i k}\right)=n_{i k} \boldsymbol{\Sigma}_{i k}^{-} \boldsymbol{y}_{i k}-n_{i k}^{2} \boldsymbol{\Sigma}_{i k}^{-} \hat{\boldsymbol{\pi}}_{i k}=0
$$

isto é,

$$
\hat{\boldsymbol{\pi}}_{i k}=\frac{1}{n_{i k}} \boldsymbol{y}_{i k} .
$$

Para $I K$ amostras de resposta multinomial com $D$ observações no tempo, o modelo linear generalizado para a $i k$-ésima observação é dado por

$$
\boldsymbol{\eta}_{i k}=g\left(\boldsymbol{\pi}_{i k}\right)=\boldsymbol{X}_{i k} \boldsymbol{\beta}_{i},
$$

em que $\boldsymbol{\eta}_{i k}$ representa o preditor linear, $g(\cdot)$ é uma função de ligação, $\boldsymbol{X}_{i k}$ é uma matriz, de dimensões $D \times m$, de covariáveis e/ou variáveis dummy para os fatores e $\boldsymbol{\beta}_{i}$ é um vetor $m \times 1$ dos parâmetros da regressão. Como funções de ligação podem ser citadas as funções de ligação logit, probit e complemento log-log.

De acordo com McCullagh \& Nelder (1989), se as categorias da resposta multinomial estão ordenadas, o modelo logit acumulado é o mais indicado por levar em consideração essa hierarquia natural entre as categorias. Desse modo, assumindose a existência de uma ordenação natural no tempo, no presente trabalho considera-se a função de ligação logit acumulado, cujo modelo é dado por

$$
g\left(\gamma_{i k, j}\right)=\log i t\left(\gamma_{i k, j}\right)=\log \left(\frac{\gamma_{i k, j}}{1-\gamma_{i k, j}}\right)=\log \left(\frac{\sum_{s=1}^{j} \pi_{i k, s}}{\sum_{s=j+1}^{D} \pi_{i k, s}}\right)=\eta_{i k, j},
$$

e para a parte sistemática tem-se um modelo de regressão com o tempo como variável explanatória e o preditor linear dado por

$$
\eta_{i k, j}=\beta_{1 i}+\beta_{2 i} t_{j}
$$

em que $\beta_{1 i}$ é o efeito baseline associado ao isolado $i$ e $\beta_{2 i}$ é o coeficiente angular da regressão linear em relação ao tempo. Em bioensaios de dose-resposta as informações 
sobre tempo letal são de grande interesse. Para o MLG com ligação logit (8) e assumindo-se o preditor linear dado em (9), o tempo letal 50 e o tempo letal 90 são dados, respectivamente, por

$$
L \hat{T}_{50}=-\hat{\beta}_{1 i} / b \hat{e t a} a_{2 i} \quad \text { e } \quad L \hat{T}_{90}=\left(\log _{e}(9)-\hat{\beta}_{1 i}\right) / \hat{\beta}_{2 i} .
$$

As estimativas de máxima verossimilhança para os parâmetros do modelo, $\hat{\boldsymbol{\beta}}_{\boldsymbol{i}}$, são obtidas usando-se o método escore de Fisher, implementado através do algoritmo de mínimos quadrados reponderados iterativamente (McCullagh \& Nelder, 1989). Para grandes amostras,

$$
\left(\hat{\boldsymbol{\beta}}_{i}-\boldsymbol{\beta}_{i}\right)^{T} \Im_{i}\left(\hat{\boldsymbol{\beta}}_{i}-\boldsymbol{\beta}_{i}\right) \stackrel{a s s}{\rightarrow} \chi_{m}^{2}
$$

sendo $\Im_{i}$ a matriz de informação de Fisher. Assim, de forma equivalente tem-se que $\hat{\boldsymbol{\beta}}_{i} \stackrel{\text { ass. }}{\rightarrow} N_{m}\left(\boldsymbol{\beta}_{i}, \Im_{i}^{-1}\right)$, sendo $\Im_{i}^{-1}$ o inverso da matriz de informação de Fisher.

\subsection{Modelo Dirichlet}

No modelo multinomial descrito em (2), as probabilidades de ocorrência das categorias na $i k$-ésima observação multinomial são consideradas como constantes. Suponha que agora, essas probabilidades sejam variáveis aleatórias positivas, $\boldsymbol{p}_{i k}=\left(p_{i k, 1}, p_{i k, 2}, \ldots, p_{i k, D}\right)^{T}$, seguindo distribuição Dirichlet (Mosimann, 1962), com função densidade de probabilidade conjunta dada por

$$
f\left(\boldsymbol{p}_{i k} ; \boldsymbol{\alpha}_{i}\right)=\frac{\Gamma\left(\sum_{j=1}^{D} \alpha_{i j}\right)}{\prod_{j=1}^{D} \Gamma\left(\alpha_{i j}\right)} p_{i k, 1}^{\alpha_{i 1}-1} p_{i k, 2}^{\alpha_{i 2}-1} \ldots p_{i k, D}^{\alpha_{i D}-1}
$$

em que $\alpha_{i}=\left(\alpha_{i 1}, \alpha_{i 2}, \ldots, \alpha_{i D}\right)^{T}, 0<p_{i k, j}<1, \alpha_{i j}>0, j=1,2, \ldots, D$ e $\sum_{j=1}^{D} p_{i k, j}=$ 1. $\mathrm{O}$ vetor de médias e a matriz de variâncias e covariâncias de $\boldsymbol{p}_{i k}$ são dados respectivamente, por

$$
E\left[\boldsymbol{p}_{i k}\right]=\frac{1}{\sum_{j=1}^{D} \alpha_{i j}} \boldsymbol{\alpha}_{i}=\boldsymbol{\pi}_{i k}
$$


e

$$
\operatorname{Var}\left[\boldsymbol{p}_{i k}\right]=\left[\operatorname{diag}\left\{\boldsymbol{\pi}_{i k}\right\}-\boldsymbol{\pi}_{i k} \boldsymbol{\pi}_{i k}^{T}\right] \rho_{i},
$$

em que $\rho_{i}=1 /\left(1+\sum_{j=1}^{D} \alpha_{i j}\right)$.

Quando $D=2$, (10) reduz-se à distribuição Beta $\left(\alpha_{i 1}, \alpha_{i 2}\right)$, em que $p_{i k, 2}=1-p_{i k, 1}, \operatorname{com} E\left(p_{i k, 1}\right)=\alpha_{i 1} /\left(\alpha_{i 1}+\alpha_{i 2}\right)$ e $\operatorname{Var}\left(p_{i k, 1}\right)=\alpha_{i 1} \alpha_{i 2} /\left[\left(\alpha_{i 1}+\alpha_{i 2}\right)^{2}(1+\right.$ $\left.\left.\alpha_{i 1}+\alpha_{i 2}\right)\right]$.

\subsection{Modelo Dirichlet - multinomial}

Nos ensaios de dose-resposta agrupados no tempo, uma alternativa para incorporar uma fonte de variação extra-multinomial ao modelo é assumir um modelo em dois estágios, semelhante ao descrito em Hinde \& Demétrio (1998a, b) para dados binomiais e de Poisson, em que ao parâmetro de média da variável resposta está associada uma distribuição. Desse modo, incondicionalmente, a variável resposta segue uma distribuição composta.

Considere que, em um primeiro estágio, $\boldsymbol{Y}_{i k} \mid \boldsymbol{p}_{i k} \sim$ multinomial $\left(n_{i k} ; \boldsymbol{p}_{i k}\right)$. com $E\left[\boldsymbol{Y}_{i k} \mid \boldsymbol{p}_{i k}\right]=n_{i k} \boldsymbol{p}_{i k}$ e matriz de variâncias e covariâncias

$$
\operatorname{Var}\left[\boldsymbol{Y}_{i k} \mid \boldsymbol{p}_{i k}\right]=n_{i k}\left[\operatorname{diag}\left\{\boldsymbol{p}_{i k}\right\}-\boldsymbol{p}_{i k} \boldsymbol{p}_{i k}^{T}\right]
$$

e, em um segundo estágio, o vetor de proporções esperadas, $\boldsymbol{p}_{\boldsymbol{i} \text {, }}$, segue uma distribuição Dirichlet como em (10). Assim, incondicionalmente, $\boldsymbol{Y}_{i k}=$ $\left(Y_{i k, 1}, Y_{i k, 2}, \ldots, Y_{i k, D}\right)^{T}$ segue uma distribuição Dirichlet-multinomial com distribuição de probabilidade dada por

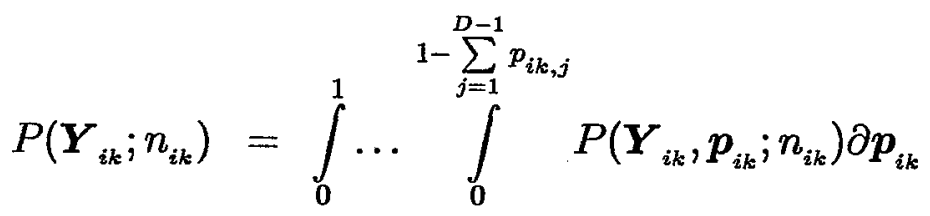

$$
\begin{aligned}
& =\int_{0}^{1} \cdots \int_{0}^{1-\sum_{j=1}^{D-1} p_{i k, j}} P\left(\boldsymbol{Y}_{i k} \mid \boldsymbol{p}_{i k} ; n_{i k}\right) f\left(\boldsymbol{p}_{i k}\right) \partial \boldsymbol{p}_{i k}
\end{aligned}
$$




$$
\begin{aligned}
& =\int_{0}^{1} \ldots \int_{0}^{1-\sum_{j=1}^{D-1} p_{i k, j}}\left(\begin{array}{c}
n_{i k} \\
y_{i k, 1} \ldots y_{i k, D}
\end{array}\right) \prod_{j=1}^{D} p_{i k, j}^{y_{i k, j}} \frac{\Gamma\left(\sum_{j=1}^{D} \alpha_{i j}\right)}{\prod_{j=1}^{D} \Gamma\left(\alpha_{i j}\right)} \prod_{j=1}^{D} p_{i k, j}^{\left(\alpha_{i j}-1\right)} \partial p_{i k} \\
& =\left(\begin{array}{c}
n_{i k} \\
y_{i k, 1} \ldots y_{i k, D}
\end{array}\right) \frac{\Gamma\left(\sum_{j=1}^{D} \alpha_{i j}\right)}{\prod_{j=1}^{D} \Gamma\left(\alpha_{i j}\right)} \int_{0}^{1} \ldots \int_{0}^{1-\sum_{j=1}^{D-1} p_{i k, j}} \prod_{j=1}^{D} p_{i k, j}^{\left(y_{i k, j}+\alpha_{i j}-1\right)} \partial p_{i k} \\
& =\left(\begin{array}{c}
n_{i k} \\
y_{i k, 1} \ldots y_{i k, D}
\end{array}\right) \frac{\Gamma\left(\sum_{j=1}^{D} \alpha_{i j}\right)}{\prod_{j=1}^{D} \Gamma\left(\alpha_{i j}\right)} \frac{\prod_{j=1}^{D} \Gamma\left(y_{i k, j}+\alpha_{i j}\right)}{\Gamma\left(n_{i k}+\sum_{j=1}^{D} \alpha_{i j}\right)}
\end{aligned}
$$

em que $0 \leq y_{i k, j} \leq n_{i k}, \alpha_{i j}>0, j=1,2, \ldots, D, \boldsymbol{Y}_{i k}=\left(Y_{i k, 1}, \ldots, Y_{i k, D}\right)^{T}$ e $\pi_{i k}=$ $\left(\pi_{i k, 1}, \ldots, \pi_{i k, D}\right)^{T}$.

Quando $D=2$, (13) fica reduzida ao modelo beta-binomial, similar ao descrito por Williams (1982) e Crowder (1978) para dados binomiais com superdispersão.

Paul et al. (1989) usaram uma forma reparametrizada de (13), fazendo $\theta_{i}=1 / \sum_{j=1}^{D} \alpha_{i j}$, na formulação de um teste de hipótese para $\theta_{i}$ com as hipóteses, $H_{o}: \theta_{i}=0($ multinomial $) \quad$ versus $\quad H_{a}: \theta_{i}>0$ (Dirichlet-multinomial), baseado na razão de verossimilhança, avaliando assim a presença da superdispersão. Utilizando-se propriedades de esperança condicional e as expressões (11) e (12), o vetor de médias e a matriz de variâncias e covariâncias de $\boldsymbol{Y}_{i k}$ são dados por

$$
E\left[\boldsymbol{Y}_{i k}\right]=E\left[E\left[\boldsymbol{Y}_{i k} \mid \boldsymbol{p}_{i k}\right]\right]=E\left[n_{i k} \boldsymbol{p}_{i k}\right]=n_{i k} \boldsymbol{\pi}_{i k}
$$

e

$$
\begin{aligned}
\operatorname{Var}\left[\boldsymbol{Y}_{i k}\right] & =E\left[\operatorname{Var}\left(\boldsymbol{Y}_{i k} \mid \boldsymbol{p}_{i k}\right)\right]+\operatorname{Var}\left[E\left(\boldsymbol{Y}_{i k} \mid \boldsymbol{p}_{i k}\right)\right] \\
& =n_{i k} E\left[\operatorname{diag}\left\{\boldsymbol{p}_{i k}\right\}-\boldsymbol{p}_{i k} \boldsymbol{p}_{i k}^{\boldsymbol{T}}\right]+n_{i k}^{2} \operatorname{Var}\left[\boldsymbol{p}_{i k}\right]
\end{aligned}
$$




$$
\begin{aligned}
& =n_{i k} E\left[\operatorname{diag}\left\{\boldsymbol{p}_{i k}\right\}\right]-n_{i k} E\left[\boldsymbol{p}_{i k} \boldsymbol{p}_{i k}^{T}\right]+n_{i k}^{2} \operatorname{Var}\left[\boldsymbol{p}_{i k}\right] \\
& =n_{i k} \operatorname{diag}\left\{\boldsymbol{\pi}_{i k}\right\}+n_{i k}\left(n_{i k}-1\right) \operatorname{Var}\left[\boldsymbol{p}_{i k}\right]-n_{i k} \boldsymbol{\pi}_{i k} \boldsymbol{\pi}_{i k}^{T} \\
& =n_{i k}\left[\operatorname{diag}\left\{\boldsymbol{\pi}_{i k}\right\}-\boldsymbol{\pi}_{i k} \boldsymbol{\pi}_{i k}^{T}\right]+n_{i k}\left(n_{i k}-1\right) \rho_{i}\left[\operatorname{diag}\left\{\boldsymbol{\pi}_{i k}\right\}-\boldsymbol{\pi}_{i k} \boldsymbol{\pi}_{i k}^{T}\right] \\
& =n_{i k}\left[\operatorname{diag}\left\{\boldsymbol{\pi}_{i k}\right\}-\boldsymbol{\pi}_{i k} \boldsymbol{\pi}_{i k}^{T}\right]\left[1+\left(n_{i k}-1\right) \rho_{i}\right]
\end{aligned}
$$

em que $\pi_{i k, j}=\alpha_{i j} /\left(\sum_{j=1}^{D} \alpha_{i j}\right)$. Aqui, $\left[1+\left(n_{i k}-1\right) \rho_{i}\right]$ é um fator de inflação da matriz de função de variâncias da distribuição multinomial, que é semelhante ao fator de heterogeneidade de Finney (1971), no qual $\rho_{i}$ é um parâmetro de superdispersão $\left(-1 /\left(n_{i k}-1\right) \leq \rho_{i} \leq 1\right)$.

\subsection{Modelo multinomial acumulado}

Em algumas situações, como para os dados apresentados na Seção 3, é mais conveniente trabalhar com o vetor de observações acumuladas.

Assumindo que $\boldsymbol{Y}_{i k} \sim$ multinomial $\left(n_{i k} ; \boldsymbol{\pi}_{i k}\right)$ e que, sendo $\boldsymbol{L}$ uma matriz, de dimensões $D \times D$, triangular inferior com valores unitários, então o vetor aleatório, de dimensões $D \times 1$, de proporções de mortalidade acumuladas, associado ao $i$-ésimo isolado na $k$-ésima repetição fica escrito como

$$
\boldsymbol{R}_{i k}=\left(R_{i k, 1}, R_{i k, 2}, \ldots, R_{i k, D}\right)^{T}=\frac{1}{n_{i k}}\left(\boldsymbol{L} \boldsymbol{Y}_{i k}\right),
$$

sendo $0 \leq R_{i k, 1} \leq R_{i k, 2} \leq \ldots \leq R_{i k, D} \leq 1$. Logo,

$$
E\left[\boldsymbol{R}_{i k}\right]=\frac{1}{n_{i k}} E\left[\boldsymbol{L} \boldsymbol{Y}_{i k}\right]=\frac{1}{n_{i k}} \boldsymbol{L} E\left[\boldsymbol{Y}_{i k}\right]=\boldsymbol{L} \pi_{i k}=\boldsymbol{\gamma}_{i k},
$$

em que $\boldsymbol{\gamma}_{i k}=\left(\gamma_{i k, 1}, \gamma_{i k, 2}, \ldots, \gamma_{i k, D}\right)^{T}$ e $\gamma_{i k, j}$ é a probabilidade acumulada esperada de um inseto morrer até o dia $j$, exposto ao $i$-ésimo isolado, na $k$-ésima repetição e utilizando-se (3),

$$
\begin{aligned}
\operatorname{Var}\left[\boldsymbol{R}_{i k}\right] & =\operatorname{Var}\left[\frac{1}{n_{i k}} \boldsymbol{L} \boldsymbol{Y}_{i k}\right]=\frac{1}{n_{i k}^{2}} \boldsymbol{L} \operatorname{Var}\left[\boldsymbol{Y}_{i k}\right] \boldsymbol{L}^{T} \\
& =\frac{1}{n_{i k}} \boldsymbol{L}\left[\operatorname{diag}\left\{\boldsymbol{\pi}_{i k}\right\}-\boldsymbol{\pi}_{i k} \boldsymbol{\pi}_{i k}^{T}\right] \boldsymbol{L}^{T}=\boldsymbol{V}\left(\boldsymbol{\gamma}_{i k}\right),
\end{aligned}
$$


sendo $\boldsymbol{V}\left(\boldsymbol{\gamma}_{\boldsymbol{i} k}\right)$ uma matriz simétrica de dimensões $D \times D$, com elementos $\boldsymbol{v}_{\boldsymbol{j} \boldsymbol{j}^{\prime}}=\boldsymbol{v}_{\boldsymbol{j}^{\prime} \boldsymbol{j}}=$ $\gamma_{i k, j}\left(1-\gamma_{i k, j^{\prime}}\right) / n_{i k},\left(1 \leq j \leq j^{\prime} \leq D\right)$.

\subsection{Modelo Dirichlet - multinomial acumulado}

\section{Supondo-se}

que, em um primeiro estágio, $\boldsymbol{Y}_{i k} \mid \boldsymbol{p}_{i k} \sim \operatorname{multinomial}\left(n_{i k} ; \boldsymbol{p}_{i k}\right)$ e que, em um segundo estágio, $\boldsymbol{p}_{\boldsymbol{i} k}$ siga uma distribuição Dirichlet, com função densidade de probabilidade dada por (10), incondicionalmente, $\boldsymbol{Y}_{\boldsymbol{i} \boldsymbol{k}}$ segue uma distribuição Dirichlet-multinomial (13). Assim, para o vetor de proporções acumuladas, $\boldsymbol{R}_{i k}$, como sendo o vetor de variável resposta e usando-se (14) e (15) tem-se que

$$
E\left[\boldsymbol{R}_{i k}\right]=E\left[\frac{1}{n_{i k}} \boldsymbol{L} \boldsymbol{Y}_{i k}\right]=\frac{1}{n_{i k}} \boldsymbol{L} E\left[E\left[\boldsymbol{Y}_{i k} \mid \boldsymbol{p}_{i k}\right]=\boldsymbol{\gamma}_{i k}\right.
$$

e

$$
\begin{aligned}
\operatorname{Var}\left[\boldsymbol{R}_{i k}\right] & =\operatorname{Var}\left[\frac{1}{n_{i k}} \boldsymbol{L} \boldsymbol{Y}_{i k}\right]=\frac{1}{n_{i k}^{2}} \boldsymbol{L} \operatorname{Var}\left[\boldsymbol{Y}_{i k}\right] \boldsymbol{L}^{T} \\
& =\frac{1}{n_{i k}} \boldsymbol{L}\left[\operatorname{diag}\left\{\boldsymbol{\pi}_{i k}\right\}-\boldsymbol{\pi}_{i k} \boldsymbol{\pi}_{i k}^{T}\right] \boldsymbol{L}^{T}\left[1+\rho_{\boldsymbol{i}}\left(\boldsymbol{n}_{i k}-1\right)\right] \\
& =\left[1+\rho_{i}\left(\boldsymbol{n}_{i k}-1\right)\right] \boldsymbol{V}\left(\boldsymbol{\gamma}_{i k}\right),
\end{aligned}
$$

em que $\boldsymbol{V}\left(\boldsymbol{\gamma}_{i k}\right)$ está definida em $(18)$, e $-1 /\left(n_{i k}-1\right) \leq \rho_{i} \leq 1$ é o parâmetro que reflete a superdispersão, semelhante ao modelo Beta-binomial de Williams (1982). Na ausência de superdispersão $\left(\rho_{i}=0\right.$, para todo $\left.i=1,2, \ldots, I\right)$ a matriz de variâncias e covariâncias definida em (20) fica reduzida à (18).

\subsection{Modelos de efeito aleatório}

O modelo Dirichlet-multinomial definido na Seção 4.5 associa uma distribuição de probabilidade ao parâmetro de média, $\boldsymbol{p}_{\boldsymbol{i} k}$, do vetor de resposta multinomial, $\boldsymbol{Y}_{i k} \mid \boldsymbol{p}_{i k}$. Uma outra forma de incorporar a superdispersão aos modelos é adicionando-se um efeito aleatório ao preditor linear, $\boldsymbol{\eta}_{i k}$, criando-se desse modo uma mudança no efeito de locação da distribuição associada à variável latente do tempo. 


\subsubsection{Modelo de efeito aleatório no intercepto - REMI}

Assumindo-se o preditor linear definido em (9) e adicionando-se um efeito aleatório ao efeito de cada isolado $i$, para cada repetição $k$, tem-se

$$
g\left(q_{i k, j}\right)=\eta_{i k, j}+\xi_{i k}=\beta_{1 i}+\beta_{2 i} t_{j}+\xi_{i k},
$$

em que $q_{i k, j}=\sum_{s=1}^{j} p_{i k, s}, E\left[\xi_{i k}\right]=0$ e $\operatorname{Var}\left[\xi_{i k}\right]=\sigma_{i}^{2}$, independentes. Um caso particular ocorre quando $\xi_{i k} \sim N\left(0, \sigma_{i}^{2}\right)$. Escrevendo-se

$$
q_{i k, j}=g^{-1}\left(\eta_{i k, j}+\xi_{i k}\right)=h\left(\eta_{i k, j}+\xi_{i k}\right)
$$

e usando-se uma aproximação em série de Taylor de primeira ordem para $h\left(\eta_{i k, j}+\xi_{i k}\right)$ em torno do preditor linear $\eta_{i k, j}$, obtém-se

$$
q_{i k, j}=h\left(\eta_{i k, j}+\xi_{i k}\right) \approx h\left(\eta_{i k, j}\right)+h^{\prime}\left(\eta_{i k, j}\right)\left(\eta_{i k, j}+\xi_{i k}-\eta_{i k, j}\right)=h\left(\eta_{i k, j}\right)+h^{\prime}\left(\eta_{i k, j}\right) \xi_{i k} .
$$

Assim

$$
\begin{aligned}
& E\left[q_{i k, j}\right] \approx E\left[h\left(\eta_{i k, j}\right)\right]+h^{\prime}\left(\eta_{i k, j}\right) E\left[\xi_{i k}\right]=h\left(\eta_{i k, j}\right)=\gamma_{i k, j} \\
& \operatorname{Var}\left[q_{i k, j}\right] \approx \operatorname{Var}\left[h\left(\eta_{i k, j}\right)\right]+\operatorname{Var}\left[h^{\prime}\left(\eta_{i k, j}\right) \xi_{i k}\right] \\
&=h^{\prime}\left(\eta_{i k, j}\right) \operatorname{Var}\left[\xi_{i k}\right] h^{\prime}\left(\eta_{i k, j}\right) \\
&=h^{\prime}\left(\eta_{i k, j}\right) h^{\prime}\left(\eta_{i k, j}\right) \sigma_{i}^{2}
\end{aligned}
$$

e

$$
\begin{aligned}
\operatorname{Cov}\left(q_{i k, j}, q_{i k, j^{\prime}}\right)= & \operatorname{Cov}\left[h\left(\eta_{i k, j}+\xi_{i k}\right), h\left(\eta_{i k, j^{\prime}}+\xi_{i k}\right)\right] \\
\approx & \operatorname{Cov}\left[h\left(\eta_{i k, j}\right)+h^{\prime}\left(\eta_{i k, j}\right) \xi_{i k}, h\left(\eta_{i k, j^{\prime}}\right)+h^{\prime}\left(\eta_{i k, j^{\prime}}\right) \xi_{i k}\right] \\
= & E\left[h\left(\eta_{i k, j}\right) h\left(\eta_{i k, j^{\prime}}\right)+h\left(\eta_{i k, j}\right) h^{\prime}\left(\eta_{i k, j^{\prime}}\right) \xi_{i k}+\right. \\
& \left.h^{\prime}\left(\eta_{i k, j}\right) h\left(\eta_{i k, j^{\prime}}\right) \xi_{i k}+h^{\prime}\left(\eta_{i k, j}\right) h^{\prime}\left(\eta_{i k, j^{\prime}}\right) \xi_{i k}^{2}\right]- \\
& E\left[h\left(\eta_{i k, j}\right)+h^{\prime}\left(\eta_{i k, j}\right) \xi_{i k}\right] E\left[h\left(\eta_{i k, j^{\prime}}\right)+h^{\prime}\left(\eta_{i k, j^{\prime}}\right) \xi_{i k}\right] \\
= & E\left[h\left(\eta_{i k, j}\right) h\left(\eta_{i k, j^{\prime}}\right)\right]+h^{\prime}\left(\eta_{i k, j}\right) h^{\prime}\left(\eta_{i k, j^{\prime}}\right) E\left[\xi_{i k}^{2}\right]- \\
& E\left[h\left(\eta_{i k, j}\right)\right] E\left[h\left(\eta_{i k, j^{\prime}}\right)\right] \\
= & \sigma_{i}^{2} h^{\prime}\left(\eta_{i k, j}\right) h^{\prime}\left(\eta_{i k, j^{\prime}}\right),
\end{aligned}
$$


no qual $h^{\prime}\left(\eta_{i k, j}\right)=\partial h\left(\eta_{i k, j}\right) / \partial \eta_{i k, j}$. Então, na notação matricial, pode-se escrever

$$
\operatorname{Var}\left[\boldsymbol{q}_{i k}\right] \approx \sigma_{i}^{2} h^{\prime}\left(\boldsymbol{\eta}_{i k}\right) h^{\prime}\left(\boldsymbol{\eta}_{i k}\right)^{T},
$$

no qual $h^{\prime}\left(\boldsymbol{\eta}_{i k}\right)=\left(\eta_{i k, 1}, \eta_{i k, 2}, \ldots, \eta_{i k, D}\right)^{T}$. Logo,

$$
E\left[\boldsymbol{R}_{i k}\right]=E\left[E\left(\boldsymbol{R}_{i k} \mid \boldsymbol{q}_{i k}\right)\right]=E\left[\boldsymbol{q}_{i k}\right] \approx \gamma_{i k},
$$

e

$$
\begin{aligned}
\operatorname{Var}\left[\boldsymbol{R}_{i k}\right] & =\operatorname{Var}\left[E\left(\boldsymbol{R}_{i k} \mid \boldsymbol{q}_{i k}\right)\right]+E\left[\operatorname{Var}\left(\boldsymbol{R}_{i k} \mid \boldsymbol{q}_{i k}\right)\right] \\
& =\operatorname{Var}\left[\boldsymbol{q}_{i k}\right]+E\left[\boldsymbol{V}\left(\boldsymbol{q}_{i k}\right)\right] \\
& \approx \sigma_{i}^{2} h^{\prime}\left(\boldsymbol{\eta}_{i k}\right) h^{\prime}\left(\boldsymbol{\eta}_{i k}\right)^{\boldsymbol{T}}+E\left[\boldsymbol{V}\left(\boldsymbol{q}_{i k}\right)\right]
\end{aligned}
$$

sendo

$$
E\left[\boldsymbol{V}\left(\boldsymbol{q}_{i k}\right)\right]=\frac{1}{n_{i k}} E\left(\begin{array}{cccc}
q_{i k, 1}-q_{i k, 1}^{2} & q_{i k, 1}-q_{i k, 1} q_{i k, 2} & \cdots & q_{i k, 1}-q_{i k, 1} q_{i k, D} \\
q_{i k, 1}-q_{i k, 1} q_{i k, 2} & q_{i k, 2}-q_{i k, 2}^{2} & \cdots & q_{i k, 2}-q_{i k, 2} q_{i k, D} \\
\cdots & \cdots & \cdots & \cdots \\
q_{i k, 1}-q_{i k, 1} q_{i k, D} & \cdots & \cdots & q_{i k, D}-q_{i k, D}^{2}
\end{array}\right)
$$

Usando-se (22) e (23) segue que

$$
\begin{aligned}
\frac{1}{n_{i k}} E\left[q_{i k, j}\right]-\frac{1}{n_{i k}} E\left[q_{i k, j} q_{i k, j^{\prime}}\right] & =\frac{1}{n_{i k}} E\left[q_{i k, j}\right]-\frac{1}{n_{i k}} \operatorname{Cov}\left(q_{i k, j}, q_{i k, j^{\prime}}\right)-\frac{1}{n_{i k}} E\left[q_{i k, j}\right] E\left[q_{i k, j^{\prime}}\right] \\
& \approx \frac{1}{n_{i k}} h\left(\eta_{i k, j}\right)-\frac{1}{n_{i k}} \sigma_{i}^{2} h^{\prime}\left(\eta_{i k, j}\right) h^{\prime}\left(\eta_{i k, j^{\prime}}\right)-\frac{1}{n_{i k}} h\left(\eta_{i k, j}\right) h\left(\eta_{i k, j^{\prime}}\right) \\
& =\frac{1}{n_{i k}} h\left(\eta_{i k, j}\right)\left(1-h\left(\eta_{i k, j^{\prime}}\right)\right)-\frac{1}{n_{i k}} \sigma_{i}^{2} h^{\prime}\left(\eta_{i k, j}\right) h^{\prime}\left(\eta_{i k, j^{\prime}}\right) .
\end{aligned}
$$

Consequentemente, (26) pode ser escrita como

$$
E\left[\boldsymbol{V}\left(\boldsymbol{q}_{i k}\right)\right] \approx \boldsymbol{V}\left(\boldsymbol{\gamma}_{i k}\right)-\frac{1}{n_{i k}} \sigma_{i}^{2} h^{\prime}\left(\boldsymbol{\eta}_{i k}\right) h^{\prime}\left(\boldsymbol{\eta}_{i k}\right)^{T},
$$

e (25) como

$$
\begin{aligned}
\operatorname{Var}\left[\boldsymbol{R}_{i k}\right] & \approx \sigma_{i}^{2} h^{\prime}\left(\boldsymbol{\eta}_{i k}\right) h^{\prime}\left(\boldsymbol{\eta}_{i k}\right)^{\boldsymbol{T}}+\boldsymbol{V}\left(\boldsymbol{\gamma}_{i k}\right)-\frac{1}{n_{i k}} \sigma_{i}^{2} h^{\prime}\left(\boldsymbol{\eta}_{i k}\right) h^{\prime}\left(\boldsymbol{\eta}_{i k}\right)^{T} \\
& =\boldsymbol{V}\left(\boldsymbol{\gamma}_{i k}\right)+\left(1-\frac{1}{n_{i k}}\right) \sigma_{i}^{2} h^{\prime}\left(\boldsymbol{\eta}_{i k}\right) h^{\prime}\left(\boldsymbol{\eta}_{i k}\right)^{T}
\end{aligned}
$$


Na ausência da superdispersão, isto é, $\sigma_{i}^{2}=0$, a matriz de variâncias e covariâncias (27) fica reduzida à mesma expressão obtida para o modelo multinomial acumulado dada em (18).

De modo análogo ao modelo Dirichlet-multinomial, é utilizado um modelo de regressão com preditor linear definido em (9) com uma função de ligação logística. Novamente são considerados os modelos com um parâmetro de superdispersão constante $\left(\sigma_{i}^{2}=\sigma^{2}\right.$ para todo $\left.i\right)$ e aqueles nos quais o parâmetro de superdispersão varia com os isolados $\left(\sigma_{i}^{2}\right)$.

\subsubsection{Modelo de efeitos aleatórios no intercepto e coeficiente angular - REMII}

O modelo definido na Seção 4.6.1 incorpora um efeito aleatório apenas ao intercepto da reta de regressão. Uma outra possibilidade seria, também, considerar os coeficientes angulares das retas como aleatórios. Desse modo, incorporando ao modelo (21) um efeito aleatório aditivo ao coeficiente angular da reta em cada isolado $i$, para cada repetição $k$, o preditor linear fica escrito como

$$
g\left(q_{i k, j}\right)=\beta_{1 i}+\xi_{i k}+\left(\beta_{2 i}+\zeta_{i k}\right) t_{j}=\eta_{i k, j}+\xi_{i k}+\zeta_{i k} t_{j},
$$

sendo que o vetor $\left(\xi_{i k}, \zeta_{i k}\right)^{T}$ tem uma distribuição com $E\left[\xi_{i k}\right]=E\left[\zeta_{i k}\right]=0$ e matriz de variâncias e covariâncias dada por

$$
\Sigma_{\nu_{i} \tau_{i} \lambda_{i}}=\left[\begin{array}{cc}
\nu_{i}^{2} & \lambda_{i} \nu_{i} \tau_{i} \\
\lambda_{i} \nu_{i} \tau_{i} & \tau_{i}^{2}
\end{array}\right],
$$

em que $-1 \leq \lambda_{i k} \leq 1$ é a correlação entre $\xi_{i k}$ e $\zeta_{i k}$. Um caso particular seria supor uma distribuição Normal bivariada para o vetor $\left(\xi_{i k}, \zeta_{i k}\right)^{T}$. Assim, escrevendo-se

$$
\begin{aligned}
q_{i k, j} & =g^{-1}\left(\eta_{i k, j}+\xi_{i k}+\zeta_{i k} t_{j}\right) \\
& =h\left(\eta_{i k, j}+\xi_{i k}+\zeta_{i k} t_{j}\right)
\end{aligned}
$$

e usando-se uma expansão em série de Taylor de primeira ordem para a probabilidade multinomial acumulada $q_{i k, j}=h\left(\eta_{i k, j}+\xi_{i k}+\zeta_{i k} t_{j}\right)$, em torno de $\eta_{i k, j}$, tem-se

$$
q_{i k, j}=h\left(\eta_{i k, j}+\xi_{i k}+\zeta_{i k} t_{j}\right)
$$




$$
\begin{aligned}
& \approx h\left(\eta_{i k, j}\right)+h^{\prime}\left(\eta_{i k, j}\right)\left(\eta_{i k, j}+\xi_{i k}+\zeta_{i k} t_{j}-\eta_{i k, j}\right) \\
& =h\left(\eta_{i k, j}\right)+h^{\prime}\left(\eta_{i k, j}\right)\left(\xi_{i k}+\zeta_{i k} t_{j}\right) .
\end{aligned}
$$

Logo,

$$
\begin{aligned}
E\left[q_{i k, j}\right] & \approx h\left(\eta_{i k, j}\right)+h^{\prime}\left(\eta_{i k, j}\right) E\left[\xi_{i k}+\zeta_{i k} t_{j}\right]=h\left(\eta_{i k, j}\right)=\gamma_{i k, j} \\
\operatorname{Var}\left[q_{i k, j}\right] & \approx \operatorname{Var}\left[h\left(\eta_{i k, j}\right)\right]+\operatorname{Var}\left[h^{\prime}\left(\eta_{i k, j}\right)\left(\xi_{i k}+\zeta_{i k} t_{j}\right)\right] \\
& =h^{\prime}\left(\eta_{i k, j}\right) \operatorname{Var}\left[\xi_{i k}+\zeta_{i k} t_{j}\right] h^{\prime}\left(\eta_{i k, j}\right) \\
& =h^{\prime}\left(\eta_{i k, j}\right) h^{\prime}\left(\eta_{i k, j}\right)\left[\operatorname{Var}\left(\xi_{i k}\right)+t_{j}^{2} \operatorname{Var}\left(\zeta_{i k}\right)+2 \operatorname{Cov}\left(\xi_{i k}, \zeta_{i k} t_{j}\right)\right] \\
& =h^{\prime}\left(\eta_{i k, j}\right) h^{\prime}\left(\eta_{i k, j}\right)\left[\nu_{i}^{2}+t_{j}^{2} \tau_{i}^{2}+2 \lambda_{i} \nu_{i} \tau_{i} t_{j}\right]
\end{aligned}
$$

e

$$
\begin{aligned}
\operatorname{Cov}\left(q_{i k, j}, q_{i k, j^{\prime}}\right)= & \operatorname{Cov}\left[h\left(\eta_{i k, j}+\xi_{i k}+\zeta_{i k} t_{j}\right), h\left(\eta_{i k, j^{\prime}}+\zeta_{i k}+\zeta_{i k} t_{j^{\prime}}\right)\right] \\
\approx & \operatorname{Cov}\left[h\left(\eta_{i k, j}\right)+h^{\prime}\left(\eta_{i k, j}\right)\left(\xi_{i k}+\zeta_{i k} t_{j}\right), h\left(\eta_{i k, j^{\prime}}\right)+h^{\prime}\left(\eta_{i k, j^{\prime}}\right)\left(\zeta_{i k}+\zeta_{i k} t_{j^{\prime}}\right)\right] \\
= & E\left[h\left(\eta_{i k, j}\right) h\left(\eta_{i k, j^{\prime}}\right)+h\left(\eta_{i k, j}\right) h^{\prime}\left(\eta_{i k, j^{\prime}}\right)\left(\xi_{i k}+\zeta_{i k} t_{j}\right)+\right. \\
& \left.h^{\prime}\left(\eta_{i k, j}\right) h\left(\eta_{i k, j^{\prime}}\right)\left(\xi_{i k}+\zeta_{i k} t_{j}\right)+h^{\prime}\left(\eta_{i k, j}\right) h^{\prime}\left(\eta_{i k, j^{\prime}}\right)\left(\xi_{i k}+\zeta_{i k} t_{j}\right)\left(\xi_{i k}+\zeta_{i k} t_{j^{\prime}}\right)\right]- \\
& E\left[h\left(\eta_{i k, j}\right)+h^{\prime}\left(\eta_{i k, j}\right)\left(\xi_{i k}+\zeta_{i k} t_{j}\right)\right] E\left[h\left(\eta_{i k, j^{\prime}}\right)+h^{\prime}\left(\eta_{i k, j^{\prime}}\right)\left(\xi_{i k}+\zeta_{i k} t_{j^{\prime}}\right)\right] \\
= & h\left(\eta_{i k, j}\right) h\left(\eta_{i k, j^{\prime}}\right)+h^{\prime}\left(\eta_{i k, j}\right) h^{\prime}\left(\eta_{i k, j^{\prime}}\right) E\left[\left(\xi_{i k}+\zeta_{i k} t_{j}\right)\left(\xi_{i k}+\zeta_{i k} t_{j^{\prime}}\right)\right]- \\
& E\left[h\left(\eta_{i k, j}\right)\right] E\left[h\left(\eta_{i k, j^{\prime}}\right)\right] \\
= & h\left(\eta_{i k, j}\right) h\left(\eta_{i k, j^{\prime}}\right)+h^{\prime}\left(\eta_{i k, j}\right) h^{\prime}\left(\eta_{i k, j^{\prime}}\right) E\left[\xi_{i k}^{2}+\zeta_{i k}^{2} t_{j} t_{j^{\prime}}+\xi_{i k} \zeta_{i k} t_{j}+\xi_{i k} \zeta_{i k} t_{j^{\prime}}\right]- \\
& h\left(\eta_{i k, j}\right) h\left(\eta_{i k, j^{\prime}}\right) \\
= & h^{\prime}\left(\eta_{i k, j}\right) h^{\prime}\left(\eta_{i k, j^{\prime}}\right)\left[\nu_{i}^{2}+\tau_{i}^{2} t_{j} t_{j^{\prime}}+\lambda_{i} \nu_{i} \tau_{i}\left(t_{j}+t_{j^{\prime}}\right)\right],
\end{aligned}
$$

sendo $h^{\prime}\left(\eta_{i k, j}\right)=\partial h\left(\eta_{i k, j}\right) / \partial \eta_{i k, j}$. Assim, em notação matricial, pode-se escrever

$$
\begin{aligned}
\operatorname{Var}\left[\boldsymbol{q}_{i k}\right] \approx & \nu_{i}^{2} h^{\prime}\left(\boldsymbol{\eta}_{i k}\right) h^{\prime}\left(\boldsymbol{\eta}_{i k}\right)^{\boldsymbol{T}}+\tau_{i}^{2}\left[h^{\prime}\left(\boldsymbol{\eta}_{i k}\right) * \boldsymbol{t}_{i k}\right]\left[h^{\prime}\left(\boldsymbol{\eta}_{i k}\right) * \boldsymbol{t}_{i k}\right]^{\boldsymbol{T}}+ \\
& \lambda_{i} \nu_{i k} \boldsymbol{\tau}_{i k} h^{\prime}\left(\boldsymbol{\eta}_{i k}\right) h^{\prime}\left(\boldsymbol{\eta}_{i k}\right)^{\boldsymbol{T}} *\left[\mathbf{1} \boldsymbol{t}_{i k}^{\boldsymbol{T}}+\boldsymbol{t}_{i k} \mathbf{1}^{\boldsymbol{T}}\right],
\end{aligned}
$$

em que 1 é um vetor coluna unitário de dimensões $\mathrm{D} \times 1, \boldsymbol{t}_{i k}^{T}=\left(t_{i k, 1}, t_{i k, 2}, \ldots, t_{i k, D}\right)$ e * indica a operação produto Hadamard (Rao, 1973). Portanto,

$$
E\left[\boldsymbol{R}_{i k}\right]=E\left(E\left[\boldsymbol{R}_{i k} \mid \boldsymbol{q}_{i k}\right)\right]=E\left[\boldsymbol{q}_{i k}\right] \approx \boldsymbol{\gamma}_{i k},
$$


e

$$
\begin{aligned}
\operatorname{Var}\left[\boldsymbol{R}_{i k}\right]= & \operatorname{Var}\left[E\left[\boldsymbol{R}_{i k} \mid \boldsymbol{q}_{i k}\right]\right]+E\left[\operatorname{Var}\left[\boldsymbol{R}_{i k} \mid \boldsymbol{q}_{i k}\right]\right] \\
= & \operatorname{Var}\left[\boldsymbol{q}_{i k}\right]+E\left[\boldsymbol{V}\left(\boldsymbol{q}_{i k}\right)\right] \\
\approx & \nu_{i}^{2} h^{\prime}\left(\boldsymbol{\eta}_{i k}\right) h^{\prime}\left(\boldsymbol{\eta}_{i k}\right)^{T}+\tau_{i}^{2}\left[h^{\prime}\left(\boldsymbol{\eta}_{i k}\right) * t_{i k}\right]\left[h^{\prime}\left(\boldsymbol{\eta}_{i k}\right) * \boldsymbol{t}_{i k}\right]^{T}+ \\
& \lambda_{i} \nu_{i} \tau_{i} h^{\prime}\left(\boldsymbol{\eta}_{i k}\right) h^{\prime}\left(\boldsymbol{\eta}_{i k}\right)^{T} *\left[\mathbf{1}_{i k}^{T}+\boldsymbol{t}_{i k} \mathbf{1}^{T}\right]+E\left[\boldsymbol{V}\left(\boldsymbol{q}_{i k}\right)\right]
\end{aligned}
$$

sendo que

$$
E\left[\boldsymbol{V}\left(\boldsymbol{q}_{i k}\right)\right]=\frac{1}{n_{i k}} E\left(\begin{array}{cccc}
q_{i k, 1}-q_{i k, 1}^{2} & q_{i k, 1}-q_{i k, 1} q_{i k, 2} & \cdots & q_{i k, 1}-q_{i k, 1} q_{i k, D} \\
q_{i k, 1}-q_{i k, 1} q_{i k, 2} & q_{i k, 2}-q_{i k, 2}^{2} & \cdots & q_{i k, 2}-q_{i k, 2} q_{i k, D} \\
\ldots & \cdots & \cdots & \cdots \\
q_{i k, 1}-q_{i k, 1} q_{i k, D} & \cdots & \cdots & q_{i k, D}-q_{i k, D}^{2}
\end{array}\right)
$$

Usando-se (29) e (30), cada elemento de (34) fica escrito como

$$
\begin{aligned}
\frac{1}{n_{i k}} E\left[q_{i k, j}\right]-\frac{1}{n_{i k}} E\left[q_{i k, j} q_{i k, j^{\prime}}\right]= & \frac{1}{n_{i k}} E\left[q_{i k, j}\right]-\frac{1}{n_{i k}} \operatorname{Cov}\left(q_{i k, j}, q_{i k, j^{\prime}}\right)-\frac{1}{n_{i k}} E\left[q_{i k, j}\right] E\left[q_{i k, j^{\prime}}\right] \\
\approx & \frac{1}{n_{i k}} h\left(\eta_{i k, j}\right)-\frac{1}{n_{i k}} h^{\prime}\left(\eta_{i k, j}\right) h^{\prime}\left(\eta_{i k, j^{\prime}}\right)\left[\nu_{i}^{2}+\tau_{i}^{2} t_{j^{\prime}} t_{j^{\prime}}+\right. \\
& \left.\lambda_{i} \nu_{i} \tau_{i}\left(t_{j}+t_{j^{\prime}}\right)\right]-\frac{1}{n_{i k}} h\left(\eta_{i k, j}\right) h\left(\eta_{i k, j^{\prime}}\right) \\
= & \frac{1}{n_{i k}} h\left(\eta_{i k, j}\right)\left[1-h\left(\eta_{i k, j^{\prime}}\right)\right]- \\
& \frac{1}{n_{i k}} h^{\prime}\left(\eta_{i k, j}\right) h^{\prime}\left(\eta_{i k, j^{\prime}}\right)\left[\nu_{i}^{2}+\tau_{i}^{2} t_{j^{\prime}} t_{j^{\prime}}+\lambda_{i} \nu_{i} \tau_{i}\left(t_{j}+t_{j^{\prime}}\right)\right]
\end{aligned}
$$

e assim

$$
\begin{gathered}
E\left[\boldsymbol{V}\left(\boldsymbol{q}_{i k}\right)\right] \approx V\left(\boldsymbol{\gamma}_{i k}\right)-\frac{1}{n_{i k}} \nu_{i}^{2} h^{\prime}\left(\boldsymbol{\eta}_{i k}\right) h^{\prime}\left(\boldsymbol{\eta}_{i k}\right)^{T}-\frac{1}{n_{i k}} \tau_{i}^{2}\left[h^{\prime}\left(\eta_{i k}\right) * \boldsymbol{t}_{i k}\right]\left[h^{\prime}\left(\boldsymbol{\eta}_{i k}\right) * \boldsymbol{t}_{i k}\right]^{T}- \\
\frac{1}{n_{i k}} \lambda_{i} \nu_{i} \tau_{i}\left[h^{\prime}\left(\boldsymbol{\eta}_{i k}\right) h^{\prime}\left(\boldsymbol{\eta}_{i k}\right)^{T}\right] *\left[\mathbf{1} t_{i k}^{T}+\boldsymbol{t}_{i k} \mathbf{1}^{T}\right]
\end{gathered}
$$

e

$$
\begin{aligned}
\operatorname{Var}\left[\boldsymbol{R}_{i k}\right] \approx & \nu_{i}^{2} h^{\prime}\left(\boldsymbol{\eta}_{i k}\right) h^{\prime}\left(\boldsymbol{\eta}_{i k}\right)^{T}+\tau_{i}^{2}\left[h^{\prime}\left(\boldsymbol{\eta}_{i k}\right) * \boldsymbol{t}_{i k}\right]\left[h^{\prime}\left(\boldsymbol{\eta}_{i k}\right) * \boldsymbol{t}_{i k}\right]^{T}+ \\
& \lambda_{i} \nu_{i} \tau_{i}\left[h^{\prime}\left(\boldsymbol{\eta}_{i k}\right) h^{\prime}\left(\boldsymbol{\eta}_{i k}\right)^{T}\right] *\left[1 t_{i k}^{T}+\boldsymbol{t}_{i k} \mathbf{1}^{T}\right]+\boldsymbol{V}\left(\boldsymbol{\gamma}_{i k}\right)-
\end{aligned}
$$




$$
\begin{aligned}
& \frac{1}{n_{i k}} \nu_{i}^{2} h^{\prime}\left(\boldsymbol{\eta}_{i k}\right) h^{\prime}\left(\boldsymbol{\eta}_{i k}\right)^{T}-\frac{1}{n_{i k}} \tau_{i}^{2}\left[h^{\prime}\left(\boldsymbol{\eta}_{i k}\right) * \boldsymbol{t}_{i k}\right]\left[h^{\prime}\left(\boldsymbol{\eta}_{i k}\right) * \boldsymbol{t}_{i k}\right]^{T}- \\
& \frac{\lambda_{i k} \nu_{i} \tau_{i}}{n_{i k}}\left[h^{\prime}\left(\boldsymbol{\eta}_{i k}\right) h^{\prime}\left(\boldsymbol{\eta}_{i k}\right)^{T}\right] *\left[1 t_{i k}^{T}+\boldsymbol{t}_{i k} \mathbf{1}^{T}\right] \\
= & \boldsymbol{V}\left(\boldsymbol{\gamma}_{i k}\right)+\left(1-\frac{1}{n_{i k}}\right) \nu_{i}^{2} h^{\prime}\left(\boldsymbol{\eta}_{i k}\right) h^{\prime}\left(\boldsymbol{\eta}_{i k}\right)^{T}+ \\
& \left(1-\frac{1}{n_{i k}}\right) \tau_{i}^{2}\left[h^{\prime}\left(\boldsymbol{\eta}_{i k}\right) * \boldsymbol{t}_{i k}\right]\left[h^{\prime}\left(\boldsymbol{\eta}_{i k}\right) * \boldsymbol{t}_{i k}\right]^{T}+ \\
& \left(1-\frac{1}{n_{i k}}\right) \lambda_{i} \nu_{i k} \tau_{i k}\left[h^{\prime}\left(\boldsymbol{\eta}_{i k}\right) h^{\prime}\left(\boldsymbol{\eta}_{i k}\right)^{T}\right] *\left[1 t_{i k}^{T}+\boldsymbol{t}_{i k} \mathbf{1}^{T}\right] .
\end{aligned}
$$

Na ausência de superdispersão, isto é, $\nu_{i}^{2}=0$ e $\tau_{i}^{2}=0$, a matriz de variâncias e covariâncias (35) é reduzida à mesma estrutura da matriz de variâncias e covariâncias do modelo multinomial acumulado dada por (18).

Como nos modelos anteriores, aqui também é utilizado um modelo de regressão com preditor linear dado por (9) e função de ligação logit. Novamente, utilizam-se os modelos com superdispersão constante $\left(\nu_{i}^{2}=\nu^{2}\right.$ e $\tau_{i}^{2}=\tau^{2}$ para todo $\left.i\right)$ e aqueles nos quais o parâmetro de superdispersão varia com os isolados $\left(\nu_{i}^{2}\right.$ e $\left.\tau_{i}^{2}\right)$.

\subsection{Equações de estimações generalizadas}

Esta Seção trata das equações de estimação generalizadas (EEG) sumarizando os principais resultados de Liang \& Zeger (1986) e Zeger \& Liang (1986). $\mathrm{O}$ método está descrito de forma resumida e a notação utilizada está baseada na mesma notação utilizada por Zeger \& Liang (1986).

$\mathrm{O}$ método das EEG está baseado na generalização do método de quase verossimilhança de Wedderburn (1974). É uma metodologia para modelos de regressão onde se tem dependência entre as observações. Possui propriedades relevantes do ponto de vista assintótico e leva a estimativas consistentes para os dos parâmetros. Muitos autores têm feito uso do método na análise de dados de natureza discreta e contínua.

Como uma extensão do método de quase verossimilhança não se faz necessária a definição completa do modelo probabilístico a ser utilizado, mas apenas 
os momentos de primeira e segunda ordem. Dessa forma fica especificada a relação entre a média e a variância do modelo adotado. A importância do método se deve ao fato de que, com exceção da suposição de distribuição Gaussiana para os dados, existem poucas alternativas para distribuições multivariadas que possam ser utilizadas para a análise de dados provenientes de bioensaios multivariados de dose-resposta.

$$
\text { Suponha que } \boldsymbol{Y}_{i}=\left(Y_{i 1}, Y_{i 2}, \ldots, Y_{i t_{i}}\right)^{T}, i=1,2, \ldots, K \text {, é o vetor de }
$$

respostas associado ao $i$-ésimo indivíduo, no qual $Y_{i t_{i}}$ associa a observação da $i$ ésima unidade amostral no tempo $t_{i}$, e $\boldsymbol{X}_{i}$ uma matriz, de dimensões $t_{i} \times m$, de covariáveis associada a $\boldsymbol{Y}_{i}$. Considere que o valor esperado para a vetor $\boldsymbol{Y}_{i}$ é dado pelo vetor, de dimensão $t_{i} \times 1$,

$$
\boldsymbol{\mu}_{i}=h\left(\boldsymbol{X}_{i} \boldsymbol{\beta}\right)
$$

no qual $\boldsymbol{\beta}$ é um vetor, de dimensão $m \times 1$, de parâmetros desconhecidos da regressão, sendo a matriz de variâncias e covariâncias associada a $\boldsymbol{Y}_{\boldsymbol{i}}$ dada por

$$
\operatorname{Var}\left(\boldsymbol{Y}_{i}\right)=\boldsymbol{V}_{i}
$$

em que $\boldsymbol{V}_{i}$ é uma matriz simétrica de dimensões $t_{i} \times t_{i}$. Essa matriz associa as covariâncias entre os tempos para a $i$-ésima unidade observacional. Para os bioensaios multivariados de dose-resposta com dados agrupados, o grupo (cluster) é considerado como a unidade observacional. Como exemplos são citados os dados dos bioensaios descritos na Seção 3.

Considerando a estrutura dos MLG descritos em (7), tem-se que a função de ligação $g$ é expressa como

$$
g\left(\boldsymbol{\eta}_{i}\right)=h^{-1}\left(\boldsymbol{\eta}_{i}\right)=h^{-1}\left(\boldsymbol{X}_{i} \boldsymbol{\beta}\right)
$$

e

$$
\partial \mu_{i} / \partial \beta^{T}=\left[\partial \mu_{i} / \partial \eta_{i}\right]\left[\partial \eta_{i} / \partial \beta^{T}\right]
$$

A função escore para o vetor de parâmetros da regressão, $\boldsymbol{\beta}$, é dada pelo sistema de equações 


$$
\begin{aligned}
U(\boldsymbol{\beta}) & =\sum_{k=1}^{K}\left[\frac{\partial \boldsymbol{\mu}_{i}}{\partial \boldsymbol{\beta}^{T}}\right]\left[\operatorname{Var}\left(\boldsymbol{Y}_{i}\right)\right]^{-1}\left(\boldsymbol{Y}_{i}-\boldsymbol{\mu}_{i}\right) \\
& =\sum_{k=1}^{K}\left[\frac{\partial \boldsymbol{\mu}_{i}}{\partial \boldsymbol{\beta}_{i}}\right] \boldsymbol{V}_{i}^{-1}\left(\boldsymbol{Y}_{i}-\boldsymbol{\mu}_{i}\right)= \\
& =\sum_{k=1}^{K} \boldsymbol{D}_{i}^{T} \boldsymbol{V}_{i} \boldsymbol{S}_{i}=0
\end{aligned}
$$

sendo $\boldsymbol{S}_{\boldsymbol{i}}=\left(\boldsymbol{Y}_{\boldsymbol{i}}-\boldsymbol{\mu}_{\boldsymbol{i}}\right)$, sendo $\boldsymbol{\mu}_{\boldsymbol{i}}$ dado por (36), $\boldsymbol{V}_{\boldsymbol{i}}$ definida em (37) e $\boldsymbol{D}_{\boldsymbol{i}}=$ $\operatorname{diag}\left\{\partial \boldsymbol{\mu}_{i} / \partial \boldsymbol{\beta}^{T}\right\}$ uma matriz de dimensões $t_{i} \times t_{i}$. Para $t_{i}=1$, para todo $i$, isto é, apenas uma medida repetida no tempo, o sistema de equações (38) fica reduzido ao sistema de equações quase- escore de Wedderburn (1974).

Para o caso de dados longitudinais, Liang \& Zeger (1986) definiram uma estrutura especial para $V_{i}$, denominando-a de matriz de trabalho, sendo dada por

$$
\boldsymbol{V}_{i}=\boldsymbol{A}_{i}^{1 / 2} \boldsymbol{R}_{i}(\boldsymbol{\alpha}) \boldsymbol{A}_{i}^{1 / 2} / \phi
$$

na qual $\boldsymbol{A}_{i}=\operatorname{diag}\left\{g\left(\mu_{i 1}\right), g\left(\mu_{i 2}\right), \ldots, g\left(\mu_{i t_{i}}\right)\right\}$ é uma matriz de dimensões $t_{i} \times t_{i}$, $\phi$ um parâmetro de escala como definido em (1) e $\boldsymbol{R}_{i}(\boldsymbol{\alpha})$ é dita ser uma matriz de correlação de trabaho,de dimensões $t_{\times} t_{i}$ que não precisa ser, necessariamente, corretamente especificada, associada a um vetor de parâmetros $\boldsymbol{\alpha}$, de dimensões $s \times 1$, considerado o mesmo para todos os indivíduos, que pode ser conhecido ou desconhecido (ver Artes, 1997 para várias maneiras de estimação de $\alpha$ ). Quando $\boldsymbol{R}_{i}(\boldsymbol{\alpha})$ é bem definida, tem-se que $\operatorname{Var}\left(\boldsymbol{Y}_{i}\right)=\boldsymbol{V}_{i}$. De forma genérica, o sistema (38) fica reescrito em função de $\boldsymbol{\beta}$ e $\boldsymbol{\alpha}$ como

$$
U(\boldsymbol{\beta}, \boldsymbol{\alpha})=\sum_{k=1}^{K} \boldsymbol{D}_{i}^{T} \boldsymbol{V}_{i} \boldsymbol{S}_{i}=0
$$

que é semelhante á função quase-escore de verossimilhança de Wedderburn (1974), com a diferença de que nas EEG $t_{i} \neq 1$ e $\boldsymbol{V}_{i}$ é função de $\boldsymbol{\beta}$ e $\boldsymbol{\alpha}$. Assim, $\hat{\boldsymbol{\beta}}$ é solução das equações em (40).

As estimativas de $\hat{\boldsymbol{\beta}}$ são obtidas de modo iterativo, à cada iteração $s$ 
através das equações

$$
\hat{\boldsymbol{\beta}}^{(s+1)}=\left(\sum_{k=1}^{K} D_{i}^{T} \boldsymbol{V}_{i}^{-1} D_{i}^{T}\right)^{-1} \sum_{k=1}^{K} D_{i}^{T} \boldsymbol{V}_{i}^{-1} \boldsymbol{z}_{i}
$$

sendo $\boldsymbol{z}_{i}=\boldsymbol{D}_{i} \hat{\boldsymbol{\beta}}^{(s)}+\left(\boldsymbol{y}_{\boldsymbol{i}}-\boldsymbol{\mu}_{\boldsymbol{i}}\right)$. O processo é repetido até a convergência com base em um critério de parada.

Sob condições gerais de regularidade, Liang \& Zeger (1986) também mostraram que a solução $\hat{\boldsymbol{\beta}}$ para a equação (38), tem distribuição assintótica normal multivariada, ou seja, para grandes valores de $k(k \rightarrow \infty), k^{1 / 2}(\hat{\boldsymbol{\beta}}-\boldsymbol{\beta}) \longrightarrow N\left(\mathbf{0}, \boldsymbol{V}_{\hat{\beta}}\right)$, com matriz de variâncias e covariâncias dada por

$$
\boldsymbol{V}_{\hat{\beta}}=\left(\sum_{k=1}^{K} \boldsymbol{D}_{i}^{T} \boldsymbol{V}_{i}^{-1} \boldsymbol{D}_{i}^{T}\right)^{-1}\left(\sum_{k=1}^{K} \boldsymbol{D}_{i}^{T} \boldsymbol{V}_{i}^{-1} \operatorname{Var}\left(\boldsymbol{Y}_{i}\right) \boldsymbol{V}_{i}^{-1} \boldsymbol{D}_{i}^{T}\right)\left(\sum_{k=1}^{K} \boldsymbol{D}_{i}^{T} \boldsymbol{V}_{i}^{-1} \boldsymbol{D}_{i}^{T}\right)^{-1}
$$

sendo $\left(\sum_{k=1}^{K} D_{i}^{T} V_{i}^{-1} D_{i}^{T}\right)^{-1}$ chamada de matriz de variâncias e covariâncias assintótica.

A matriz de variâncias e covariâncias $V_{\hat{\beta}}$ é conhecida na literatura como estimador sanduíche ou matriz de variâncias e covariâncias robusta. Autores como Liang \& Zeger (1986), Vonesh \& Chinchilli (1997) e Artes (1997) discutem, de forma exaustiva, as especificações das estruturas de correlação da matriz $\boldsymbol{R}_{i}(\boldsymbol{\alpha})$.

\subsection{Estimação dos parâmetros dos modelos para os ensaios com os cupins e os ácaros}

As técnicas de equações de estimação generalizadas (EEG) descritas acima, foram utilizadas para estimar os parâmetros do modelo de regressão, nas diferentes parametrizações para superdispersão através dos modelos multinomial, Dirichlet-multinomial, REMI e REMII.

Considerando-se os dados descritos na Seção 3 e utilizando-se a notação definida nas Seções 4.1 a 4.6, as EEG para o vetor dos parâmetros do modelo de regressão definido em (9) são dadas por 


$$
\begin{aligned}
U\left(\boldsymbol{\beta}_{i}\right) & =\sum_{k=1}^{K}\left[\frac{\partial \boldsymbol{\gamma}_{i k}}{\partial \boldsymbol{\beta}_{i}^{T}}\right]\left[\operatorname{Var}\left(\boldsymbol{R}_{i k}\right)\right]^{-1}\left(\boldsymbol{r}_{i k}-\boldsymbol{\gamma}_{i k}\right) \\
& =\sum_{k=1}^{K}\left[\frac{\partial \boldsymbol{\gamma}_{i k}}{\partial \boldsymbol{\eta}_{i k}}\right]\left[\frac{\partial \boldsymbol{\eta}_{i k}}{\partial \boldsymbol{\beta}_{i k}^{T}}\right]\left[\operatorname{Var}\left(\boldsymbol{R}_{i k}\right)\right]^{-1}\left(\boldsymbol{r}_{i k}-\boldsymbol{\gamma}_{i k}\right) \\
& =\sum_{k=1}^{K} \boldsymbol{X}_{i k}^{T}\left[\frac{\partial \boldsymbol{\eta}_{i k}}{\partial \boldsymbol{\gamma}_{i k}}\right]^{-1}\left[\boldsymbol{V}_{i k}^{\mathrm{OD}}\right]^{-1}\left(\boldsymbol{r}_{i k}-\boldsymbol{\gamma}_{i k}\right)=0,
\end{aligned}
$$

sendo $\boldsymbol{X}_{t k}$ uma matriz de covariáveis que incluem as funções no tempo, de dimensões $D \times m, \partial \eta_{i k} / \partial \boldsymbol{\gamma}_{i k}=\operatorname{diag}\left\{\partial \boldsymbol{\eta}_{i k} / \partial \boldsymbol{\gamma}_{i k}\right\}$ uma matriz diagonal de dimensões $D \times D$, $\boldsymbol{V}_{i k}^{\text {OD }}$ uma matriz simétrica de dimensões $D \times D$ dada por (20), (27) ou (35) e $\boldsymbol{r}_{i k}$ um vetor de proporções acumuladas dos insetos mortos até o dia $j$, de dimensão $D \times 1$. Mesmo quando $\boldsymbol{V}_{i k}^{\text {oD }}$ não for bem especificada, $E\left[U\left(\boldsymbol{\beta}_{i}\right)\right]=0$ e então a solução de (42) é consistente.

Fixando-se $\rho_{i}, \sigma_{i}^{2}$ ou $\left(\nu_{i}^{2}, \tau_{i}^{2}, \lambda_{i}\right)$, a estimativa $\hat{\boldsymbol{\beta}}_{i}$ é encontrada através do procedimento de mínimos quadrados reponderados iterativamente, no qual as equações iterativas para $\boldsymbol{\beta}_{\boldsymbol{i}}$ são dadas por

$$
\hat{\beta}_{i}^{(s+1)}=\left(\sum_{k=1}^{K} X_{i k}^{T} W_{i k}^{\mathrm{OD}} \boldsymbol{X}_{i k}\right)^{-1} \sum_{k=1}^{K} \boldsymbol{X}_{i k}^{T} W_{i k}^{\mathrm{OD}} z_{i k}
$$

com

$$
W_{i k}^{\mathrm{OD}}=\left[\frac{\partial \boldsymbol{\eta}_{i k}}{\partial \boldsymbol{\gamma}_{i k}}\right]^{-1}\left[\boldsymbol{V}_{i k}^{\mathrm{OD}}\right]^{-1}\left[\frac{\partial \boldsymbol{\eta}_{i k}}{\partial \boldsymbol{\gamma}_{i k}}\right]^{-1},
$$

sendo $\boldsymbol{z}_{i k}=\boldsymbol{X}_{i k} \hat{\boldsymbol{\beta}}_{i}^{(s)}+\left[\partial \boldsymbol{\eta}_{i k} / \partial \boldsymbol{\gamma}_{i k}\right]\left(\boldsymbol{r}_{i k}-\boldsymbol{\gamma}_{i k}\right)$, com matriz de covariância assintótica dada por

$$
\operatorname{Var}\left(\hat{\boldsymbol{\beta}}_{i}\right)=\left(\sum_{k=1}^{K} \boldsymbol{X}_{i k}^{T}\left[\frac{\partial \boldsymbol{\eta}_{i k}}{\partial \boldsymbol{\gamma}_{i k}}\right]^{-1}\left[\boldsymbol{V}_{i k}^{\mathrm{OD}}\right]^{-1}\left[\frac{\partial \boldsymbol{\eta}_{i k}}{\partial \boldsymbol{\gamma}_{i k}}\right]^{-1} \boldsymbol{X}_{i k}\right)^{-1}
$$

Liang and Zeger (1986) mostraram que sob condições de regularidade, $\hat{\boldsymbol{\beta}}_{i}$, a solução de (42), é consistente com distribuição assintótica normal com matriz de covariâncias dada por

$$
\operatorname{Var}\left(\hat{\boldsymbol{\beta}}_{i}\right)=M_{0}^{-1} M_{1} M_{0}^{-1}
$$


onde $M_{0}^{-1}$ é dada por (45) e

$$
M_{1}=\left(\sum_{k=1}^{K} \boldsymbol{X}_{i k}^{T}\left[\frac{\partial \boldsymbol{\eta}_{i k}}{\partial \boldsymbol{\gamma}_{i k}}\right]^{-1}\left[\boldsymbol{V}_{i k}^{\mathrm{OD}}\right\}^{-1}\left(\boldsymbol{r}_{i k}-\boldsymbol{\gamma}_{i k}\right)\left(\boldsymbol{r}_{i k}-\boldsymbol{\gamma}_{i k}\right)^{T}\left[\boldsymbol{V}_{i k}^{\mathrm{OD}}\right]^{-1}\left[\frac{\partial \boldsymbol{\eta}_{i k}}{\partial \boldsymbol{\gamma}_{i k}}\right]^{-1} \boldsymbol{X}_{i k}\right)
$$

Como já foi mencionado anteriormente, essa matriz (16varrobusta) é chamada de estimador sanduíche ou estimador robusto da $\operatorname{Var}\left(\boldsymbol{\beta}_{\boldsymbol{i}}\right)$. O termo robusto refere-se ao fato da robustez com relação à escolha de $\boldsymbol{V}_{i k}^{\mathrm{OD}}$.

Para o modelo Dirichlet-multinomial, foi usado o método dos momentos (Williams, 1982; Moore, 1987) para estimar o parâmetro de superdispersão em (20). Igualando-se a estatística Qui-quadrado de Pearson $\chi^{2}$ ao seu valor esperado, isto é, resolvendo-se a equação $X^{2}=E\left(X^{2}\right)$, tem-se a equação de estimação de $\rho_{i}$, dada por

$$
\sum_{k=1}^{K}\left(\boldsymbol{r}_{i k}-\boldsymbol{\gamma}_{i k}\right)^{T}\left[\boldsymbol{V}_{i k}^{-1}\right]^{\mathrm{OD}}\left(\boldsymbol{r}_{i k}-\boldsymbol{\gamma}_{i k}\right)=K D-m
$$

ou, similarmente

$$
\sum_{k=1}^{K} \frac{\left(\boldsymbol{r}_{i k}-\boldsymbol{\gamma}_{i k}\right)^{T} \boldsymbol{V}^{-1}\left(\boldsymbol{\gamma}_{i k}\right)\left(\boldsymbol{r}_{i k}-\boldsymbol{\gamma}_{i k}\right)}{1+\left(n_{i k}-1\right) \hat{\rho}_{i}}=K D-m
$$

Considerando-se que, para os dados utilizados neste trabalho, $n_{i k}=n$, para todo $i$, o estimador de $\rho_{i}$ é dado por

$$
\hat{\rho}_{i}=\frac{1}{n-1}\left(\sum_{k=1}^{K} \frac{\left(\boldsymbol{r}_{i k}-\boldsymbol{\gamma}_{i k}\right)^{T} \boldsymbol{V}^{-1}\left(\boldsymbol{\gamma}_{i k}\right)\left(\boldsymbol{r}_{i k}-\boldsymbol{\gamma}_{i k}\right)}{K D-m}-1\right) .
$$

Atualizando-se o valor de $\hat{\rho}_{i}$ em (43) e (44), a estimativa $\hat{\beta}_{i}$ é encontrada utilizandose (43) e depois ela é usada para estimar $\hat{\rho}_{i}$ novamente, de modo que o procedimento é repetido iterativamente até a ocorrência da convergência do processo com base em um critério de parada.

Usando-se o método dos momentos para estimar $\sigma_{i}^{2}$ no modelo REMI, é necessário utilizar a equação iterativa (O'Hara Hines \& Lawless, 1993)

$$
\sigma_{i}^{2}(s+1)=\frac{1}{K-1} \sum_{k=1}^{K} \frac{\sigma_{i}^{2}(s)\left[\boldsymbol{h}^{\prime}\left(\boldsymbol{\eta}_{i k}\right)^{\boldsymbol{T}}\left(\boldsymbol{r}_{i k}-\boldsymbol{\gamma}_{i k}\right)\right]^{2}}{\boldsymbol{h}^{\prime}\left(\boldsymbol{\eta}_{i k}\right)^{T} \boldsymbol{V}_{i k}^{\mathrm{OD}}(s) \boldsymbol{h}^{\prime}\left(\boldsymbol{\eta}_{i k}\right)}
$$


sendo $\boldsymbol{V}_{i k}^{\mathrm{OD}}(s)$, dada por (27), evaluated at the current estimate of $\sigma_{i}^{2}$, i.e., $\sigma_{i}^{2}(s)$. De maneira similar, as equações iterativas para a estimação de $\nu_{i}^{2}$ e $\tau_{i}^{2}$ no modelo REMII são dadas por

$$
\begin{gathered}
\nu_{i}^{2}(s+1)=\frac{1}{K-1} \sum_{k=1}^{K} \frac{\nu_{i}^{2}(s)\left[\boldsymbol{h}^{\prime}\left(\boldsymbol{\eta}_{i k}\right)^{T}\left(\boldsymbol{r}_{i k}-\boldsymbol{\gamma}_{i k}\right)\right]^{2}}{\boldsymbol{h}^{\prime}\left(\boldsymbol{\eta}_{i k}\right)^{T} V_{i k}^{\mathrm{OD}}(s) \boldsymbol{h}^{\prime}\left(\boldsymbol{\eta}_{i k}\right)}, \\
\tau_{i}^{2}(s+1)=\frac{1}{K-1} \sum_{k=1}^{K} \frac{\tau_{i}^{2}(s)\left[\left(\boldsymbol{h}^{\prime}\left(\boldsymbol{\eta}_{i k}\right)^{T} \times \boldsymbol{t}_{i k}\right)\left(\boldsymbol{r}_{i k}-\boldsymbol{\gamma}_{i k}\right)\right]^{2}}{\left[\boldsymbol{h}^{\prime}\left(\boldsymbol{\eta}_{i k}\right) \times \boldsymbol{t}_{i k}\right]^{T} \boldsymbol{V}_{i k}^{\mathrm{OD}}(s)\left[\boldsymbol{h}^{\prime}\left(\boldsymbol{\eta}_{i k}\right) \times \boldsymbol{t}_{i k}\right]},
\end{gathered}
$$

sendo $\boldsymbol{V}_{i k}^{\mathrm{OD}}(s)$ dada por (35), atualizada em $\nu_{i}^{2}(s)$ e $\tau_{i}^{2}(s)$. As estimativas para $\lambda_{i}$ foram obtidas do ajuste de um modelo para cada réplica $k$, fornecendo assim valores de $\hat{\beta}_{1 i k}$ e $\hat{\beta}_{2 i k}$, e posteriormente calculando-se $\hat{\lambda}_{i}$ como sendo a correlação amostral entre $\hat{\beta}_{1 i k}$ e $\hat{\beta}_{2 i k}$. Esses valores de $\lambda_{i}$ foram considerados como fixos nos procedimentos iterativos subsequentes. Novamente, foi feita a iteração entre a estimação de $\sigma_{i}^{2}$ ou $\left(\nu_{i}^{2}, \tau_{i}^{2}\right)$ e a atualização de $\boldsymbol{\beta}_{i}$ usando-se (43) e $\boldsymbol{z}_{i k}$ em (44) até a convergência do processo com base em algum critério de parada.

Para os modelos nos quais considerou-se um parâmetro de superdispersão que não variasse com os isolados, $i$, ou seja, $\rho_{i}=\rho$ (modelo DM), $\sigma_{i}^{2}=\sigma^{2}$ (modelo REMI) e $\nu_{i}^{2}=\nu^{2}$ e $\tau_{i}^{2}=\tau^{2}$ (modelo REMII), as equações de estimação foram dadas por

$$
\begin{gathered}
\hat{\rho}=\frac{1}{n-1}\left(\sum_{i=1}^{I} \sum_{k=1}^{K} \frac{\left(\boldsymbol{r}_{i k}-\boldsymbol{\gamma}_{i k}\right)^{T} \boldsymbol{V}^{-1}\left(\boldsymbol{\gamma}_{i k}\right)\left(\boldsymbol{r}_{i k}-\boldsymbol{\gamma}_{i k}\right)}{I K D-q}-1\right) \\
\sigma^{2}(s+1)=\frac{1}{I K-1} \sum_{i=1}^{I} \sum_{k=1}^{K} \frac{\sigma^{2}(s)\left[\boldsymbol{h}^{\prime}\left(\boldsymbol{\eta}_{i k}\right)^{T}\left(\boldsymbol{r}_{i k}-\boldsymbol{\gamma}_{i k}\right)\right]^{2}}{\boldsymbol{h}^{\prime}\left(\boldsymbol{\eta}_{i k}\right)^{T} \boldsymbol{V}_{i k}^{\mathrm{OD}}(s) \boldsymbol{h}^{\prime}\left(\boldsymbol{\eta}_{i k}\right)} \\
\nu^{2}(s+1)=\frac{1}{I K-1} \sum_{i=1}^{I} \sum_{k=1}^{K} \frac{\nu^{2}(s)\left[\boldsymbol{h}^{\prime}\left(\boldsymbol{\eta}_{i k}\right)^{T}\left(\boldsymbol{r}_{i k}-\boldsymbol{\gamma}_{i k}\right)\right]^{2}}{\boldsymbol{h}^{\prime}\left(\boldsymbol{\eta}_{i k}\right)^{T} V_{i k}^{\mathrm{OD}}(s) \boldsymbol{h}^{\prime}\left(\boldsymbol{\eta}_{i k}\right)} \\
\tau^{2}(s+1)=\frac{1}{I K-1} \sum_{i=1}^{I} \sum_{k=1}^{K} \frac{\tau^{2}(s)\left[\left(\boldsymbol{h}^{\prime}\left(\boldsymbol{\eta}_{i k}\right)^{T} \times \boldsymbol{t}_{i k}\right)\left(\boldsymbol{r}_{i k}-\boldsymbol{\gamma}_{i k}\right)\right]^{2}}{\left[\boldsymbol{h}^{\prime}\left(\boldsymbol{\eta}_{i k}\right) \times \boldsymbol{t}_{i k}\right]^{T} V_{i k}^{\mathrm{OD}}(s)\left[\boldsymbol{h}^{\prime}\left(\boldsymbol{\eta}_{i k}\right) \times \boldsymbol{t}_{i k}\right]}
\end{gathered}
$$




\section{RESULTADOS E DISCUSSÃO}

\subsection{Resultados para o ensaio com o cupim $H$. tenuis:}

Os resultados apresentados nesta Secção foram obtidos utilizando-se os dados do ensaio com o cupim $H$. tenuis, conforme descrito na Seção 3 com o uso do preditor linear descrito em (9).

Observa-se que pelas expressões (17), (19), (24) e (32) os valores esperados para o vetor de resposta multinomial acumulada é o mesmo entre todos os modelos: multinomial, DM, REMI e REMII, não importando assim qual seja a suposição feita para estrutura da matriz de variâncias e covariâncias, por exemplo (18), (20), (27) ou (35).

Como esperado, as estimativas dos parâmetros do modelo de regressão, $\beta_{1 i}$ e $\beta_{2 i}$, são as mesmas para todos os modelos mas os desvios padrões das estimativas mudam de acordo com os modelos. As Figuras 1 a 8 mostram os valores observados e a curva estimada para os 142 isolados utilizados no ensaio dos cupins.

Pode-se observar pelas Figuras 1 a 8 que existe uma variabilidade considerável no intercepto e no coeficiente angular entre as réplicas para alguns isolados. Este fato foi observado para muitos dos 142 isolados, mostrando assim que é razoável utilizar modelos que permitam efeitos aleatórios tanto para o intercepto $\left(\beta_{1 i}\right)$ como também o coeficiente angular $\left(\beta_{2 i}\right)$.

Pelo diagrama de dispersão dos desvios padrões dos $\hat{\boldsymbol{\beta}}^{\prime} \mathrm{s}$ do modelo

multinomial versus os desvios padrões dos $\hat{\boldsymbol{\beta}}_{\text {s }}^{\prime}$ dos modelos DM, REMI e REMII, 
visto na Figura $9(a, b)$, nota-se que ignorar a presença da variação extra-multinomial pode conduzir a uma subestimação nas estimativas dos desvios padrões dos $\hat{\beta}^{\prime}$ s. Esse fato tende a subestimar o erro padrão verdadeiro das estimativas dos efeitos dos isolados. Também observa-se que o modelo Dirichlet-multinomial parece superestimar o d.p. $(\hat{\boldsymbol{\beta}})$ quando comparado com o modelo multinomial e os dois modelos de efeitos aleatórios, REMI e REMII.

A Figura $9(c, d)$ mostra a igualdade dos resultados da matriz de variâncias e covariâncias robusta entre todos os modelos. Isto não foi um resultado esperado, mas parece ser causado pela estrutura especial do predictor linear (9), que possui apenas o dia como variável regressora. O mesmo padrão de comportamento foi encontrado com os dados apresentados por O'Hara Hines (1993), quando excluídas todas as covariáveis do modelo e ajustando somente a regressão no tempo. Para explorar este comportamento, foi feito um pequeno estudo de simulação para a variável dia, em cada réplica/isolado, através da adição de pequenas peturbações aleatórias com distribuição normal (média zero e variância 0.5). Fazendo com que a matriz de delineamento $X$ não ficasse constante entre as réplicas/isolado. Usando-se, então, esta variável modificada como variável explanatória foi possível verificar que a matriz de covariância robusta apresentou diferentes resultados para os quatro diferentes modelos considerados. Consequentemente, está claro que este comportamento está relacionado com a inclusão da tendência estritamente linear no tempo, isto é, em cima da resposta multinomial ordenada.

A Figura $10(a-g)$ mostra como as distribuições das estimativas dos parâmetros de suprdispersão apresentam um comportamento assimétrico. Isto também pode ser visto na Tabela 5. Se considerarmos os modelos com superdispersão constante tem-se que a estimativa do parâmetro de superdispersão para $\rho$, é 0.1621 (modelo DM), para $\sigma^{2}, 0.7645$ (modelo REMI), para $\nu^{2}, 0.2520$ e para $\tau^{2}$ é 0.0439 (modelo REMII). Também é possível observar, pela Figura 10 ( $a$ ) e pelas estatísticas apresentadas na Tabela 5 , que não existe evidência de superdispersão $(\hat{\rho}<0)$ em quase metade dos isolados analisados. Isso parece indicar que o modelo 
DM não modela a superdispersão de maneira satisfatória.

Tabela 5: Sumário das estatísticas descritivas para os parâmetros de superdispersão dos modelos DM, REMI e REMII, nos quais a superdispersão varia entre os isolados, considerando-se os dados das Tabelas 2 a 9 .

\begin{tabular}{ccccccc}
\hline \multirow{2}{*}{ Modelo } & \multirow{2}{*}{$\begin{array}{c}\text { Parâmetro de } \\
\text { superdispersão }\end{array}$} & média (d.p.) & Mediana & Min. & Max. & CV \% \\
\cline { 3 - 7 } & $\rho_{i}$ & $0,0053(0,0852)$ & $-0,0069$ & $-0,1252$ & 0,3436 & 1607,55 \\
\hline DM & $\sigma_{i}^{2}$ & $0,9514(0,6496)$ & 0,7924 & $9,23 \mathrm{e}-08$ & 3,0357 & 68,29 \\
\hline \multirow{2}{*}{ REMII } & $\nu_{i}^{2}$ & $0,4265(0,7875)$ & $1,8 \mathrm{e}-07$ & $1,06 \mathrm{e}-10$ & 4,4623 & 184,64 \\
& $\tau_{i}^{2}$ & $0,0413(0,1382)$ & 0,0235 & $6,41 \mathrm{e}-06$ & 1,6499 & 334,22 \\
\hline
\end{tabular}

Na Figura $10(h, i)$ nota-se que, para muitos dos 142 isolados utilizados no ensaio dos cupins, o tempo letal médio $\left(L T_{50}\right)$ é superior a 8 dias, o último dia avaliado no ensaio. Esse fato é ainda mais agravante quando se observa o tempo letal $90, L T_{90}$, indicando a necessidade de continuação do experimento durante mais dias, para assim obter-se uma estimativa melhor do modelo. Segundo os entomologistas, isto é contraposto ao fato de que após alguns dias, os cupins tendem a criar uma resistência aos isolados, criando assim uma resistência natural ao patógeno, mostrando que o prolongamento do ensaio por mais dias pode vir a prejudicar o ajuste dos modelos. O pesquisador está interessado em agrupar os isolados em três grupos: alta virulência, média virulência e virulência fraca para poder identificar os patógenos mais virulentos que possam ser usados nos experimentos de campo. Assim, um método estatístico adequado para agrupar os isolados seria um que fosse robusto à falta de informação para a estimação de alguns modelos. A análise feita neste trabalho não está completa há necessidade de se continuá-la.

A Figure $10(j)$ mostra que a correlação entre o intercepto e o coeficiente angular da reta é negativa e isto é confirmado pelo histograma (g). Na Figura 10 (k) vê-se que existe um isolado discrepante no conjunto e, quando eliminado, a Figura $10(l)$ mostra que há um grupo grande de isolados com variabilidade muito 
pequena para o intercepto, também confirmado pelas Figuras 1 a 8 . Isso mostra a necessidade de diferentes modelos para grupos diferentes de isolados, isto é, modelos com e sem efeitos aleatórios para o intercepto.

Apesar de não dispormos de um teste estatístico que discrimine entre os modelos considerados neste trabalho, recomendamos o uso do modelo com efeitos aleatórios no intercepto e no coeficiente angular da reta (REMII), por ter apresentado resultados mais consistentes e coerentes. 
Tabela 6: Estimativas dos parâmetros da regressão e dos parâmetros de superdispersão para o ensaio com os cupins.

\begin{tabular}{|c|c|c|c|c|c|c|c|c|c|c|}
\hline Isolados & $i$ & $\hat{\beta}_{1}$ & $\hat{\beta}_{2}$ & $\hat{\rho}$ & $\hat{\sigma}^{2}$ & $\hat{\nu}^{2}$ & $\hat{\tau}^{2}$ & $\hat{\lambda}$ & $\hat{L T}_{50}$ & $\hat{L T}_{90}$ \\
\hline 848 & 1 & $-2,3502$ & 0,4389 & 0,0014 & 0,1002 & 0,0000 & 0,0041 & $-0,4815$ & 5,3546 & 10,3605 \\
\hline 787 & 2 & $-3,3515$ & 0,5874 & 0,0079 & 0,9051 & 0,6597 & 0,0351 & $-0,9825$ & 5,7059 & 9,4466 \\
\hline 1003 & 3 & $-6,1158$ & 0,7182 & $-0,0902$ & 0,0874 & 0,0000 & 0,0024 & $-0,9924$ & 8,5160 & 11,5755 \\
\hline 1006 & 4 & $-4,1212$ & 0,6059 & 0,0183 & 1,4414 & 0,8178 & 0,0460 & $-0,5558$ & 6,8020 & 10,4285 \\
\hline 1024 & 5 & $-5,0843$ & 0,4381 & $-0,1225$ & 0,4737 & 0,5325 & 0,0073 & $-0,7275$ & 11,6063 & 16,6221 \\
\hline 852 & 6 & $-2,8732$ & 0,3911 & 0,1607 & 1,8984 & 1,6447 & 0,0576 & $-0,1761$ & 7,3464 & 12,9645 \\
\hline 879 & 7 & $-6,5716$ & 0,7280 & $-0,1150$ & 0,3481 & 0,0001 & 0,0066 & $-0,8439$ & 9,0263 & 12,0442 \\
\hline 883 & 8 & $-6,2150$ & 0,6853 & $-0,0840$ & 1,2016 & 1,1562 & 0,0216 & $-0,6596$ & 9,0696 & 12,2759 \\
\hline 885 & 9 & $-5,3840$ & 0,5547 & $-0,0570$ & 2,7049 & 1,5116 & 0,0657 & $-0,7337$ & 9,7059 & 13,6669 \\
\hline 957 & 10 & $-4,7804$ & 0,6651 & $-0,0480$ & 1,6963 & 0,6073 & 0,0510 & $-0,8098$ & 7,1870 & 10,4904 \\
\hline 1028 & 11 & $-4,0902$ & 0,9799 & 0,1053 & 0,3239 & 0,0000 & 0,0238 & $-0,9821$ & 4,1743 & 6,4167 \\
\hline 732 & 12 & $-3,8235$ & 0,7219 & 0,0007 & 1,4281 & 0,9050 & 0,0634 & $-0,9242$ & 5,2965 & 8,3401 \\
\hline 743 & 13 & $-5,0598$ & 0,9515 & 0,0036 & 0,8161 & 0,0000 & 0,0342 & $-0,9754$ & 5,3177 & 7,6270 \\
\hline 745 & 14 & $-4,6689$ & 0,4925 & 0,0160 & 1,9725 & 2,5029 & 0,0198 & 0,0913 & 9,4795 & 13,9407 \\
\hline 767 & 15 & $-3,8781$ & 0,5825 & 0,0055 & 1,8233 & 1,5113 & 0,0531 & $-0,8407$ & 6,6573 & 10,4292 \\
\hline 823 & 16 & $-3,8332$ & 0,5840 & $-0,0467$ & 0,5593 & 0,1882 & 0,0199 & $-0,9588$ & 6,5634 & 10,3255 \\
\hline 855 & 17 & $-4,2173$ & 0,5219 & 0,0453 & 1,6943 & 1,3180 & 0,0430 & $-0,5534$ & 8,0805 & 12,2906 \\
\hline 857 & 18 & $-4,5906$ & 0,5800 & $-0,0748$ & 0,5975 & 0,4336 & 0,0152 & $-0,6867$ & 7,9150 & 11,7034 \\
\hline 876 & 19 & $-6,2790$ & 0,9859 & $-0,1020$ & 0,4638 & 0,5663 & 0,0071 & $-0,9507$ & 6,3688 & 8,5974 \\
\hline 878 & 20 & $-4,2551$ & 0,4939 & $-0,0848$ & 0,1628 & 0,0000 & 0,0040 & $-0,6373$ & 8,6150 & 13,0636 \\
\hline 602 & 21 & $-2,2006$ & 0,3995 & $-0,0138$ & 0,5908 & 0,7235 & 0,0139 & $-0,6134$ & 5,5087 & 11,0089 \\
\hline 616 & 22 & $-2,1575$ & 0,1833 & 0,0032 & 3,0358 & 3,7650 & 0,0096 & $-0,1607$ & 11,7718 & 23,7605 \\
\hline 741 & 23 & $-2,5113$ & 0,2642 & $-0,0175$ & 2,0760 & 2,3479 & 0,0012 & $-0,8526$ & 9,5039 & 17,8190 \\
\hline 784 & 24 & $-2,9728$ & 0,3666 & $-0,0835$ & 0,6391 & 0,7041 & 0,0002 & $-0,9339$ & 8,1081 & 14,1008 \\
\hline 822 & 25 & $-2,3576$ & 0,3563 & $-0,0751$ & 0,3932 & 0,4344 & 0,0105 & $-0,6903$ & 6,6162 & 12,7823 \\
\hline 841 & 26 & $-4,2310$ & 0,7434 & $-0,0427$ & 0,6427 & 0,0037 & 0,0223 & $-0,9873$ & 5,6910 & 8,6465 \\
\hline 843 & 27 & $-3,8631$ & 0,6311 & $-0,0062$ & 1,2171 & 1,4333 & 0,0248 & $-0,9399$ & 6,1211 & 9,6027 \\
\hline 845 & 28 & $-3,8843$ & 0,7628 & $-0,0122$ & 0,1673 & 0,1984 & 0,0044 & $-0,6946$ & 5,0919 & 7,9721 \\
\hline 738 & 29 & $-4,3116$ & 0,8282 & $-0,0252$ & 0,3832 & 0,0000 & 0,0186 & $-0,9932$ & 5,2059 & 7,8589 \\
\hline 742 & 30 & $-3,8756$ & 0,5860 & 0,0514 & 1,1690 & 0,0000 & 0,0341 & $-0,9141$ & 6,6135 & 10,3630 \\
\hline 744 & 31 & $-4,4579$ & 0,8073 & $-0,0355$ & 0,7038 & 0,0000 & 0,0283 & $-0,9867$ & 5,5222 & 8,2440 \\
\hline 747 & 32 & $-5,3426$ & 1,2548 & $-0,0232$ & 0,7860 & 0,6539 & 0,0473 & $-0,9434$ & 4,2577 & 6,0087 \\
\hline 748 & 33 & $-3,7840$ & 0,9552 & 0,2970 & 0,6319 & 0,0000 & 0,0574 & $-0,9773$ & 3,9613 & 6,2615 \\
\hline 750 & 34 & $-5,3655$ & 1,0172 & 0,0006 & 0,6339 & 0,0000 & 0,0262 & $-0,9745$ & 5,2748 & 7,4349 \\
\hline 752 & 35 & $-5,8800$ & 1,1949 & $-0,0019$ & 0,4308 & 0,0000 & 0,0194 & $-0,9797$ & 4,9211 & 6,7600 \\
\hline 755 & 36 & $-3,9278$ & 0,8044 & 0,0555 & 1,7022 & 1,5698 & 0,0726 & $-0,9010$ & 4,8830 & 7,6146 \\
\hline 756 & 37 & $-5,8459$ & 1,2670 & 0,2479 & 0,7470 & 0,0000 & 0,0411 & $-0,9838$ & 4,6140 & 6,3482 \\
\hline 764 & 38 & $-4,1486$ & 0,9309 & $\mathbf{0 , 0 8 3 1}$ & 0,2304 & 0,0000 & 0,0159 & $-0,9982$ & 4,4564 & 6,8166 \\
\hline 573 & 39 & $-5,0225$ & 0,6901 & $-0,0953$ & 0,0017 & 0,0000 & 0,0002 & $-0,9820$ & 7,2780 & 10,4619 \\
\hline 591 & 40 & $-4,3897$ & 0,5702 & $-0,0529$ & 0,7039 & 0,6556 & 0,0160 & $-0,6772$ & 7,6982 & 11,5515 \\
\hline
\end{tabular}


Continuação da Tabela (6). Estimativas dos parâmetros da regressão e dos parâmetros de superdispersão para o ensaio com os cupins.

\begin{tabular}{|c|c|c|c|c|c|c|c|c|c|c|}
\hline Isolados & $i$ & $\hat{\beta}_{1}$ & $\hat{\beta}_{2}$ & $\hat{\boldsymbol{p}}$ & $\hat{\sigma}^{2}$ & $\hat{\nu}^{2}$ & $\hat{\tau}^{2}$ & $\hat{\boldsymbol{\lambda}}$ & $\hat{L T} T_{50}$ & $\hat{L T}_{90}$ \\
\hline 598 & 41 & $-4,3692$ & 0,4996 & $-0,0794$ & 0,1653 & 0,0000 & 0,0044 & $-0,9462$ & 8,7448 & 13,1425 \\
\hline 600 & 42 & $-4,2268$ & 0,7096 & 0,0139 & 1,3458 & 0,0000 & 0,0390 & $-0,7079$ & 5,9565 & 9,0529 \\
\hline 604 & 43 & $-4,3965$ & 0,6278 & $-0,0005$ & 1,6793 & 0,0000 & 0,0402 & $-0,7041$ & 7,0030 & 10,5029 \\
\hline 666 & 44 & $-5,7857$ & 0,8408 & $-0,0845$ & 0,5895 & 3,0252 & 0,0244 & $-0,4718$ & 6,8810 & 9,4941 \\
\hline 587 & 45 & $-5,8294$ & 1,4015 & 0,3289 & 0,1981 & 0,0000 & 0,0149 & $-0,9984$ & 4,1593 & 5,7271 \\
\hline 619 & 46 & $-3,9422$ & 0,4957 & $-0,0423$ & 0,5202 & 0,0000 & 0,0110 & $-0,4149$ & 7,9521 & 12,3842 \\
\hline 781 & 47 & $-4,1491$ & 0,6979 & $-0,0809$ & 0,0000 & 0,0000 & 0,0001 & $-0,9662$ & 5,9454 & 9,0938 \\
\hline 783 & 48 & $-4,0658$ & 0,5808 & $-0,0220$ & 0,8341 & 0,0000 & 0,0213 & $-0,9696$ & 7,0003 & 10,7833 \\
\hline 786 & 49 & $-3,1883$ & 0,4537 & 0,0223 & 0,8330 & 0,0000 & 0,0192 & $-0,1944$ & 7,0280 & 11,8714 \\
\hline 665 & 50 & $-3,2201$ & 0,5915 & $-0,0012$ & 0,3950 & 0,0000 & 0,0162 & $-0,9360$ & 5,4444 & 9,1593 \\
\hline 705 & 51 & $-3,6529$ & 0,4789 & $-0,0468$ & 0,5033 & 0,0000 & 0,0123 & 0,4915 & 7,6269 & 12,2145 \\
\hline 753 & 52 & $-5,0935$ & 0,9430 & 0,1104 & 1,6044 & 0,0000 & 0,0638 & $-0,9854$ & 5,4014 & 7,7314 \\
\hline 778 & 53 & $-4,7418$ & 0,9267 & 0,1210 & 1,9713 & 0,0000 & 0,0805 & $-0,9936$ & 5,1169 & 7,4880 \\
\hline 607 & 54 & $-4,7828$ & 0,5790 & $-0,1129$ & 0,2390 & 0,5030 & 0,0010 & $-0,9798$ & 8,2611 & 12,0563 \\
\hline 609 & 55 & $-4,4787$ & 0,8892 & 0,0322 & 1,2701 & 0,0000 & 0,0573 & $-0,9349$ & 5,0366 & 7,5076 \\
\hline 615 & 56 & $-5,6953$ & 1,1227 & $-0,0079$ & 0,9791 & 0,0000 & 0,0404 & $-0,8852$ & 5,0731 & 7,0302 \\
\hline 617 & 57 & $-3,2452$ & 0,5579 & $-0,0587$ & 0,9525 & 0,0000 & 0,0321 & $-0,7708$ & 5,8169 & 9,7554 \\
\hline 634 & 58 & $-3,2542$ & 0,7532 & $-0,0066$ & 1,0306 & 0,0000 & 0,0493 & $-0,8727$ & 4,3204 & 7,2376 \\
\hline 635 & 59 & $-3,1523$ & 0,6871 & $-0,0271$ & 0,5126 & 0,0000 & 0,0257 & $-0,8804$ & 4,5877 & 7,7855 \\
\hline 643 & 60 & $-3,8204$ & 0,6165 & 0,0263 & 1,9437 & 0,0000 & 0,0613 & $-0,8992$ & 6,1969 & 9,7610 \\
\hline 651 & 61 & $-3,0309$ & 0,5867 & $-0,0445$ & 0,4912 & 0,0000 & 0,0231 & $-0,9296$ & 5,1663 & 8,9116 \\
\hline 268 & 62 & $-2,3811$ & 0,5119 & 0,1292 & 0,5515 & 0,0000 & 0,0307 & $-0,9778$ & 4,6518 & 8,9443 \\
\hline 565 & 63 & $-2,9215$ & 0,2881 & $-0,0334$ & 0,2915 & 0,0000 & 0,0101 & $-0,8899$ & 10,1424 & 17,7703 \\
\hline 571 & 64 & $-2,3333$ & 0,3197 & 0,1298 & 0,4114 & 0,0000 & 0,0130 & 0,0244 & 7,2994 & 14,1731 \\
\hline 509 & 65 & $-3,8060$ & 0,3884 & $-0,0170$ & 0,5622 & 0,0000 & 0,0123 & $-0,7604$ & 9,7993 & 15,4566 \\
\hline 515 & 66 & $-4,6571$ & 0,5123 & $-0,0170$ & 1,2518 & 0,0000 & 0,0250 & 0,1978 & 9,0913 & 13,3805 \\
\hline 520 & 67 & $-3,1633$ & 0,4508 & $-0,0084$ & 1,3905 & 0,0000 & 0,0341 & $-0,8928$ & 7,0164 & 11,8900 \\
\hline 531 & 68 & $-4,8528$ & 0,6514 & $-0,1026$ & 0,1632 & 0,0000 & 0,0045 & $-0,9408$ & 7,4500 & 10,8232 \\
\hline 535 & 69 & $-4,1853$ & 0,4860 & 0,0163 & 1,7645 & 0,0000 & 0,0364 & $-0,8048$ & 8,6123 & 13,1337 \\
\hline 545 & 70 & $-4,1045$ & 0,6388 & $-0,0623$ & 0,3604 & 2,1861 & 0,0245 & $-0,9562$ & 6,4250 & 9,8644 \\
\hline 553 & 71 & $-3,7249$ & 0,4602 & $-0,0826$ & 0,4776 & 0,0000 & 0,0118 & $-0,8485$ & 8,0937 & 12,8681 \\
\hline 556 & 72 & $-3,1012$ & 0,4271 & 0,0466 & 1,4056 & 0,0000 & 0,0387 & $-0,8674$ & 7,2605 & 12,4046 \\
\hline 560 & 73 & $-5,1146$ & 0,5901 & $-0,0868$ & 0,6874 & 0,0000 & 0,0140 & $-0,7841$ & 8,6673 & 12,3907 \\
\hline 585 & 74 & $-4,2238$ & 0,5619 & $-0,0722$ & 0,3626 & 0,0000 & 0,0098 & $-0,9169$ & 7,5170 & 11,4274 \\
\hline 532 & 75 & $-3,0731$ & 0,5120 & 0,0567 & 2,6213 & 0,0000 & 0,0744 & $-0,8275$ & 6,0025 & 10,2942 \\
\hline 554 & 76 & $-4,0899$ & 0,5852 & $-0,0703$ & 1,1885 & 0,0000 & 0,0299 & $-0,4663$ & 6,9890 & 10,7438 \\
\hline 555 & 77 & $-3,9582$ & 0,5361 & $-0,0038$ & 1,1378 & 0,0000 & 0,0255 & $-0,8363$ & 7,3829 & 11,4812 \\
\hline 558 & 78 & $-6,0455$ & 0,8175 & $-0,0970$ & 0,3025 & 0,0000 & 0,0066 & $-0,9283$ & 7,3951 & 10,0828 \\
\hline 562 & 79 & $-4,2295$ & 0,7661 & $-0,0028$ & 0,7399 & 0,0000 & 0,0255 & $-0,9552$ & 5,5207 & 8,3886 \\
\hline 566 & 80 & $-3,7211$ & 0,5385 & $-0,0156$ & 0,5537 & 0,0000 & 0,0132 & $-0,8225$ & 6,9097 & 10,9897 \\
\hline
\end{tabular}


Continuação da Tabela (6). Estimativas dos parâmetros da regressão e dos parâmetros de superdispersão para o ensaio com os cupins.

\begin{tabular}{|c|c|c|c|c|c|c|c|c|c|c|}
\hline Isolados & $i$ & $\hat{\beta}_{1}$ & $\hat{\beta}_{2}$ & $\hat{\rho}$ & $\hat{\sigma}^{2}$ & $\hat{\nu}^{2}$ & $\hat{\tau}^{2}$ & $\hat{\boldsymbol{\lambda}}$ & $\hat{L T} T_{50}$ & $\hat{L T} T_{90}$ \\
\hline 569 & 81 & $-3,5183$ & 0,4703 & $-0,0338$ & 0,5836 & 0,0000 & 0,0151 & 0,3208 & 7,4816 & 12,1541 \\
\hline 572 & 82 & $-3,8818$ & 0,5640 & $-0,0955$ & 0,5317 & 0,0000 & 0,0125 & $-0,7208$ & 6,8821 & 10,7777 \\
\hline 574 & 83 & $-4,3804$ & 0,5412 & $-0,0374$ & 0,3470 & 0,0000 & 0,0073 & $-0,8605$ & 8,0937 & 12,1536 \\
\hline 448 & 84 & $-4,5038$ & 0,8672 & 0,1759 & 2,1276 & 0,0000 & 0,0882 & $-0,9794$ & 5,1935 & 7,7273 \\
\hline 534 & 85 & $-4,2581$ & 0,7560 & 0,1177 & 2,2957 & 0,0000 & 0,0789 & $-0,9772$ & 5,6320 & 8,5382 \\
\hline 536 & 86 & $-4,3102$ & 0,6129 & 0,0642 & 2,6585 & 0,0000 & 0,0613 & $-0,9947$ & 7,0319 & 10,6166 \\
\hline 538 & 87 & $-4,2929$ & 0,7138 & $-0,0549$ & 0,7590 & 0,0000 & 0,0226 & $-0,0448$ & 6,0137 & 9,0917 \\
\hline 542 & 88 & $-3,5931$ & 0,6461 & 0,0383 & 2,0382 & 0,0000 & 0,0663 & $-0,9152$ & 5,5608 & 8,9613 \\
\hline 544 & 89 & $-3,6968$ & 0,5168 & $-0,0530$ & 1,4000 & 0,0000 & 0,0308 & $-0,7848$ & 7,1525 & 11,4037 \\
\hline 548 & 90 & $-3,2747$ & 0,5901 & 0,0803 & 2,5824 & 0,0000 & 0,0920 & $-0,7487$ & 5,5493 & 9,2727 \\
\hline 549 & 91 & $-3,9146$ & 0,5949 & 0,0030 & 1,4122 & 0,0000 & 0,0344 & $-0,5507$ & 6,5806 & 10,2742 \\
\hline 550 & 92 & $-3,6647$ & 0,6624 & $-0,0155$ & 0,7420 & 0,0000 & 0,0223 & $-0,8769$ & 5,5326 & 8,8498 \\
\hline 391 & 93 & $-3,4796$ & 0,5760 & 0,0212 & 0,9933 & 0,0000 & 0,0345 & $-0,8216$ & 6,0408 & 9,8553 \\
\hline 484 & 94 & $-4,6409$ & 0,8097 & 0,0014 & 1,7443 & 0,0000 & 0,0525 & $-0,9608$ & 5,7315 & 8,4451 \\
\hline 485 & 95 & $-4,6295$ & 0,4504 & $-0,0616$ & 0,4763 & 0,0000 & 0,0100 & $-0,5361$ & 10,2791 & 15,1577 \\
\hline 489 & 96 & $-2,4679$ & 0,5456 & 0,0536 & 1,6253 & 0,0000 & 0,0713 & $-0,5858$ & 4,5233 & 8,5505 \\
\hline 498 & 97 & $-3,9887$ & 1,0540 & 0,0097 & 1,3594 & 0,0000 & 0,0860 & $-0,9464$ & 3,7844 & 5,8691 \\
\hline 512 & 98 & $-4,4088$ & 0,9725 & $-0,0025$ & 0,5766 & 0,6567 & 0,0001 & $-0,9776$ & 4,5335 & 6,7929 \\
\hline 524 & 99 & $-3,6058$ & 0,7858 & 0,1924 & 1,6887 & 0,0000 & 0,0940 & $-0,9679$ & 4,5889 & 7,3852 \\
\hline 529 & 100 & $-2,8159$ & 0,5072 & 0,0908 & 1,4675 & 0,0000 & 0,0537 & $-0,2347$ & 5,5524 & 9,8848 \\
\hline 476 & 101 & $-1,7891$ & 0,5076 & $-0,0570$ & 1,1592 & 1,9218 & 0,0058 & $-0,5506$ & 3,5244 & 7,8528 \\
\hline 672 & 102 & $-2,0205$ & 0,4310 & $-0,0731$ & 0,3218 & 0,0000 & 0,0137 & $-0,7775$ & 4,6880 & 9,7860 \\
\hline 760 & 103 & $-2,6047$ & 0,7993 & 0,0602 & 1,0080 & 0,0000 & 0,0966 & $-0,8812$ & 3,2588 & 6,0078 \\
\hline 969 & 104 & $-2,3132$ & 0,4771 & $-0,0537$ & 0,5927 & 0,0000 & 0,0233 & $-0,8726$ & 4,8488 & 9,4546 \\
\hline 986 & 105 & $-2,8615$ & 0,7347 & 0,0434 & 1,3235 & 0,0000 & 0,0709 & $-0,0625$ & 3,8945 & 6,8850 \\
\hline 987 & 106 & $-1,8251$ & 0,5912 & 0,0039 & 2,0533 & 4,4623 & 1,6500 & $-0,3515$ & 3,0871 & 6,8036 \\
\hline 988 & 107 & $-2,6929$ & 0,5990 & $-0,0292$ & 0,3539 & 0,0000 & 0,0120 & $-0,8598$ & 4,4959 & 8,1643 \\
\hline 1010 & 108 & $-3,8334$ & 0,5214 & $-0,0696$ & 0,4857 & 0,0000 & 0,0121 & $-0,4915$ & 7,3524 & 11,5665 \\
\hline 800 & 109 & $-2,5244$ & 0,5219 & $-0,0362$ & 0,7023 & 0,0000 & 0,0224 & $-0,5827$ & 4,8372 & 9,0475 \\
\hline 868 & 110 & $-2,7763$ & 0,5962 & $-0,0141$ & 0,7987 & 0,0000 & 0,0366 & $-0,6925$ & 4,6565 & 8,3417 \\
\hline 888 & 111 & $-3,5894$ & 0,6794 & 0,0027 & 0,7140 & 0,0000 & 0,0253 & $-0,9496$ & 5,2833 & 8,5174 \\
\hline 900 & 112 & $-2,0387$ & 0,7275 & 0,0358 & 1,8125 & 1,8373 & 0,0000 & $-0,9801$ & 2,8023 & 5,8225 \\
\hline 940 & 113 & $-3,4532$ & 0,2117 & $-0,1252$ & 0,0731 & 2,0074 & 0,0420 & $-0,9761$ & 16,3148 & 26,6957 \\
\hline 620 & 114 & $-4,0565$ & 0,7377 & 0,1389 & 1,5818 & 0,0000 & 0,0590 & $-0,9735$ & 5,4991 & 8,4778 \\
\hline 641 & 115 & $-4,7853$ & 0,7482 & $-0,0682$ & 0,4611 & 0,0000 & 0,0117 & $-0,8284$ & 6,3960 & 9,3327 \\
\hline 649 & 116 & $-4,3485$ & 0,6979 & $-0,0224$ & 0,9195 & 0,0000 & 0,0274 & $-0,7729$ & 6,2305 & 9,3787 \\
\hline 663 & 117 & $-3,6254$ & 0,5376 & $-0,0340$ & 0,3553 & 0,0000 & 0,0108 & $-0,9442$ & 6,7430 & 10,8297 \\
\hline 669 & 118 & $-3,6984$ & 0,6328 & 0,0117 & 1,2572 & 0,0000 & 0,0389 & $-0,8681$ & 5,8441 & 9,3161 \\
\hline 694 & 119 & $-3,5761$ & 0,4994 & $-0,0091$ & 1,5425 & 0,0000 & 0,0409 & $-0,6709$ & 7,1604 & 11,5600 \\
\hline 704 & 120 & $-3,5529$ & 0,5952 & $-0,0163$ & 0,8933 & 0,0000 & 0,0255 & $-0,8803$ & 5,9696 & 9,6614 \\
\hline
\end{tabular}


Continuação da Tabela (6). Estimativas dos parâmetros da regressão e dos parâmetros de superdispersão para o ensaio com os cupins.

\begin{tabular}{c|c|cc|ccccc|cc}
\hline Isolados & $i$ & $\hat{\beta}_{1}$ & $\hat{\beta}_{2}$ & $\hat{\rho}$ & $\hat{\sigma}^{2}$ & $\hat{\nu}^{2}$ & $\hat{\tau}^{2}$ & $\hat{\boldsymbol{\lambda}}$ & $\hat{L T}_{50}$ & $\hat{L}_{90}$ \\
\hline 292 & 121 & $-2,9594$ & 0,4808 & $-0,0071$ & $\mathbf{0 , 8 2 6 5}$ & 1,1923 & $\mathbf{0 , 0 1 3 7}$ & $-\mathbf{0 , 8 1 1 6}$ & $\mathbf{6 , 1 5 5 2}$ & 10,7251 \\
501 & 122 & $-2,1803$ & 0,2696 & 0,0418 & 1,0166 & 1,4288 & 0,0227 & $-0,7779$ & 8,0876 & 16,2382 \\
579 & 123 & $-2,3181$ & 0,4011 & 0,0542 & 1,0348 & 1,4483 & 0,0269 & $-0,6621$ & 5,7797 & 11,2581 \\
612 & 124 & $-2,7749$ & 0,5394 & 0,0055 & 1,0179 & 1,4696 & 0,0156 & $-0,3976$ & 5,1441 & 9,2173 \\
618 & 125 & $-3,1855$ & 0,8986 & 0,0983 & 1,4914 & 1,7243 & 0,1277 & $-0,9225$ & 3,5449 & 5,9901 \\
473 & 126 & $-1,8566$ & 0,5900 & 0,0059 & 0,2236 & 0,0000 & 0,0218 & $-0,9526$ & 3,1469 & 6,8712 \\
494 & 127 & $-3,8007$ & 0,8840 & $-0,0150$ & 0,3186 & 0,4241 & 0,0147 & $-0,6090$ & 4,2995 & 6,7852 \\
499 & 128 & $-2,5485$ & 0,6068 & 0,0286 & 0,5603 & 0,7960 & 0,0197 & $-0,9642$ & 4,1999 & 7,8208 \\
500 & 129 & $-2,4750$ & 0,6195 & $-0,0582$ & 0,2503 & 0,2923 & 0,0003 & $-0,6756$ & 3,9949 & 7,5414 \\
469 & 130 & $-1,9323$ & 0,5420 & $-0,0118$ & 1,0413 & 1,0599 & 0,0000 & $-0,9143$ & 3,5648 & 7,6183 \\
pl-61 & 131 & $-5,5007$ & 0,7249 & $-0,0840$ & 1,2754 & 0,0000 & 0,0278 & $-0,7780$ & 7,5879 & 10,6188 \\
pl-63 & 132 & $-5,0970$ & 1,0794 & 0,0501 & 0,3208 & 0,0000 & 0,0170 & $-0,9959$ & 4,7220 & 6,7576 \\
197 & 133 & $-4,5877$ & 0,6358 & $-0,0449$ & 0,7353 & 0,8404 & 0,0191 & $-0,7714$ & 7,2158 & 10,6717 \\
210 & 134 & $-3,6965$ & 0,7401 & 0,1693 & 0,8697 & 0,0000 & 0,0380 & $-0,9933$ & 4,9946 & 7,9634 \\
309 & 135 & $-3,4189$ & 0,4980 & 0,1240 & 1,0899 & 1,1177 & 0,0365 & $-0,4958$ & 6,8650 & 11,2769 \\
341 & 136 & $-3,1020$ & 0,5859 & 0,0668 & 1,7929 & 1,9977 & 0,0772 & $-0,4042$ & 5,2946 & 9,0448 \\
353 & 137 & $-4,4634$ & 0,5275 & $-0,1059$ & 0,5116 & 0,6027 & 0,0118 & $-0,8717$ & 8,4616 & 12,6271 \\
282 & 138 & $-3,0411$ & 0,5468 & 0,0039 & 0,1210 & 0,0000 & 0,0047 & $-0,8942$ & 5,5621 & 9,5807 \\
428 & 139 & $-2,0373$ & 0,3382 & 0,1612 & 1,0772 & 1,1325 & 0,0001 & 0,2930 & 6,0244 & 12,5218 \\
457 & 140 & $-2,1047$ & 0,4570 & 0,3436 & 0,8131 & 0,6683 & 0,0525 & $-0,8861$ & 4,6051 & 9,4126 \\
458 & 141 & $-3,1927$ & 0,4877 & 0,0730 & 1,0095 & 0,9263 & 0,0384 & $-0,5903$ & 6,5469 & 11,0526 \\
462 & 142 & $-1,6533$ & 0,2499 & 0,1278 & 0,0185 & 0,0000 & 0,0026 & $-0,9218$ & 6,6172 & 15,4113 \\
\hline
\end{tabular}




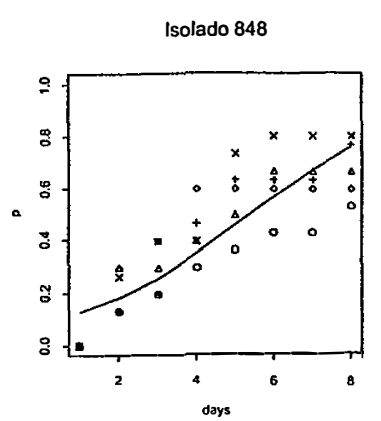

Isolado 1024

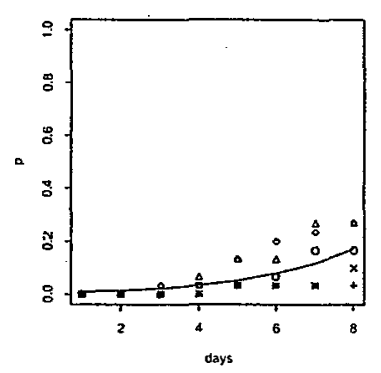

Isolado 885

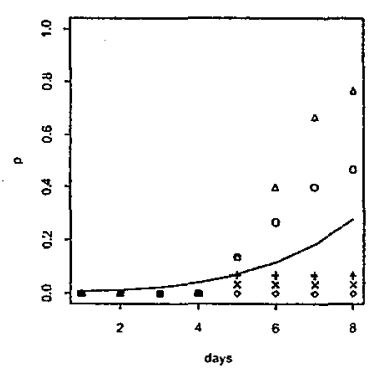

isolado 743

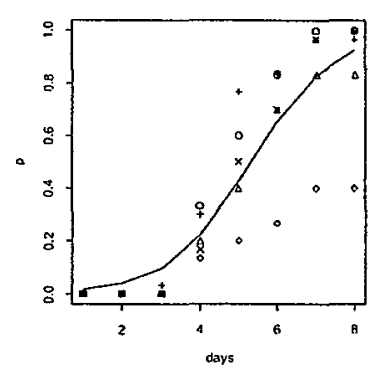

Isolado 855

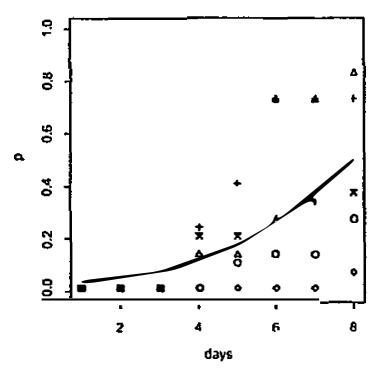

isolado 787

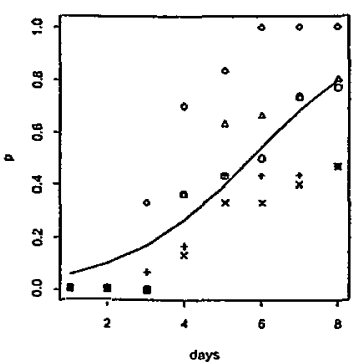

Isolado 852

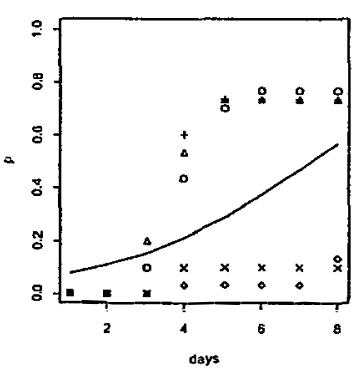

Isolado 957

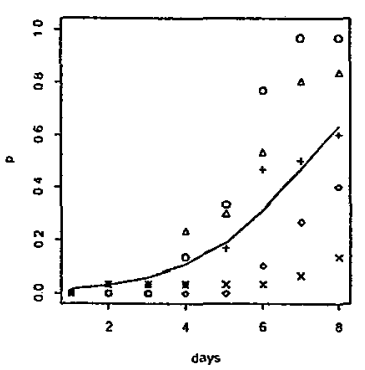

Isolado 745

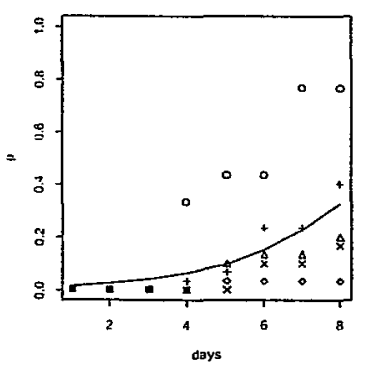

Isolado 857

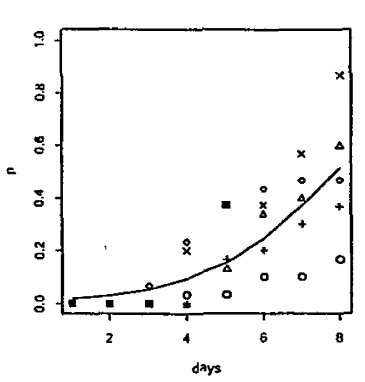

Isolado 1003

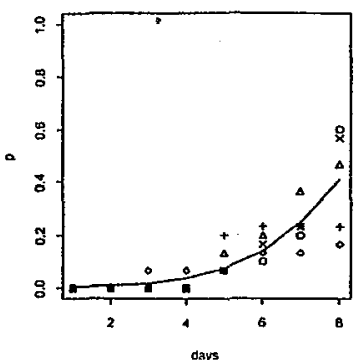

Isolado 879

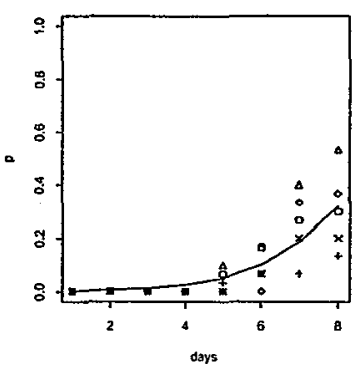

Isolado 1028

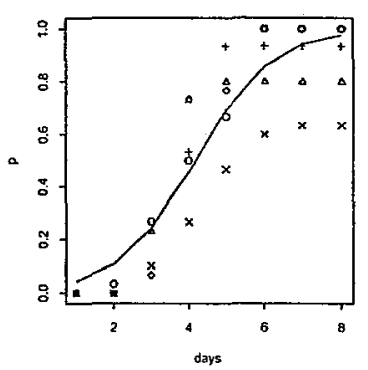

Isolado 767

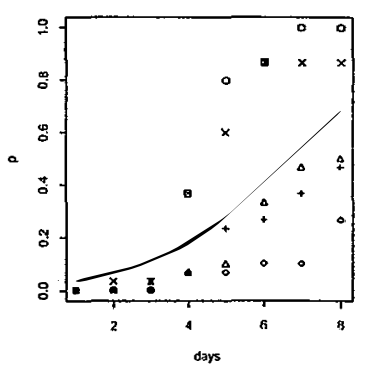

Isolado 876

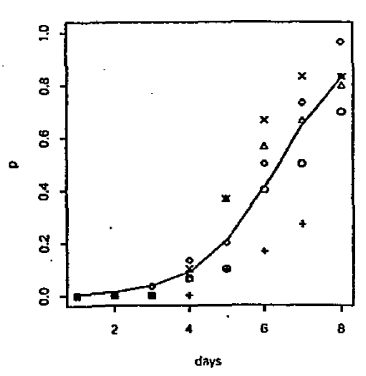

Isolado 1006

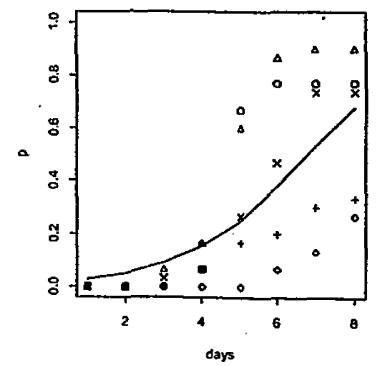

Isolado 883

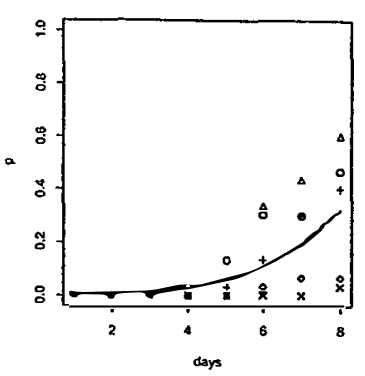

Isolado 732

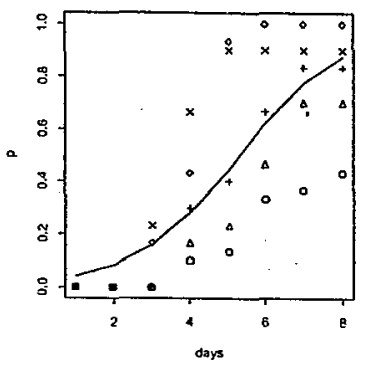

Isolado 823

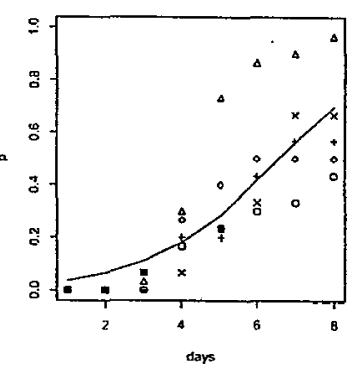

Isolado 878

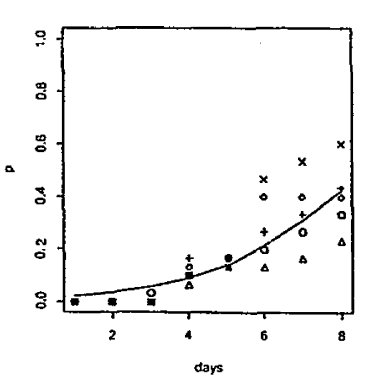

Figura 1: Proporção da mortalidade acumulada durante os 8 dias, para vinte isolados do experimento com o cupim $H$. tenuis. 


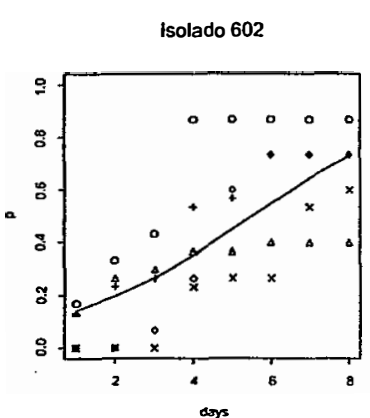

Isolado 822

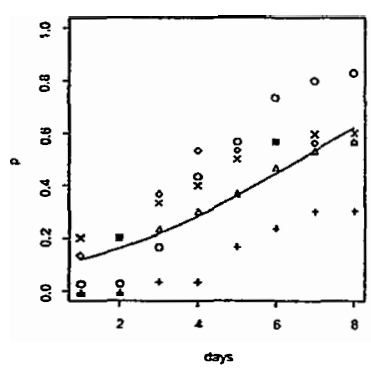

Isolado 738

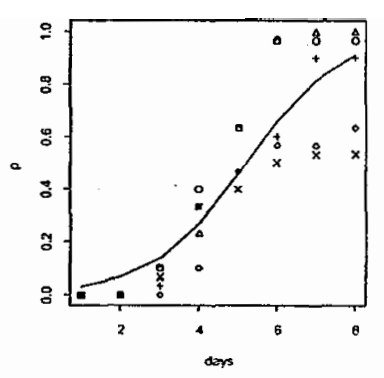

Isolado 748

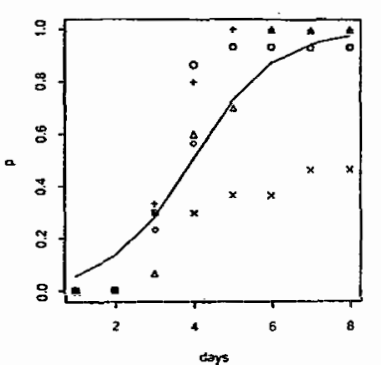

Isolado 756

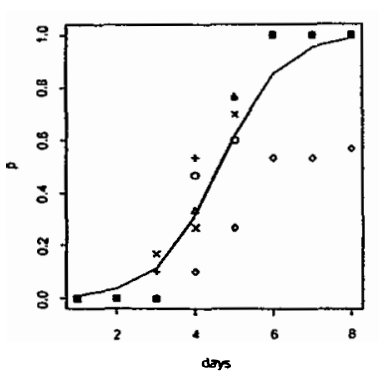

Isolado 616

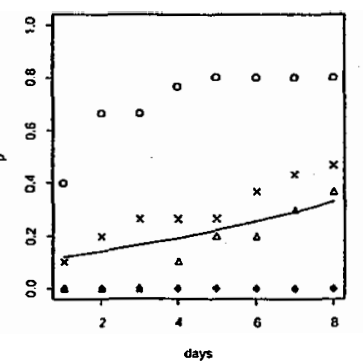

Isolado 841

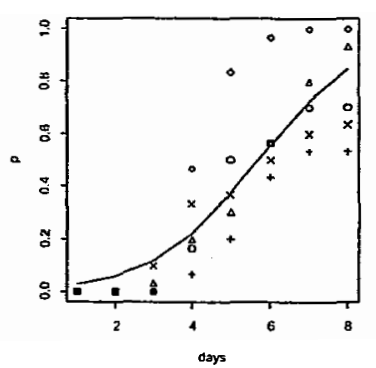

Isolado 742

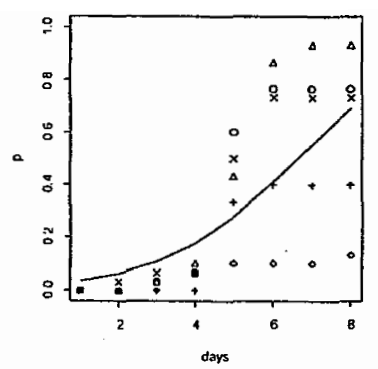

Isolado 750

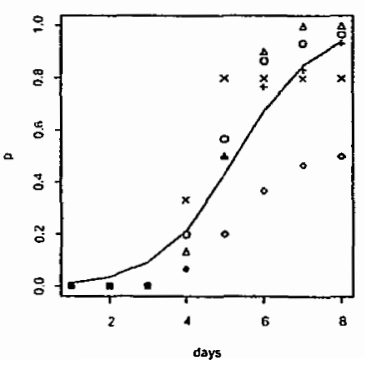

Isolado 764

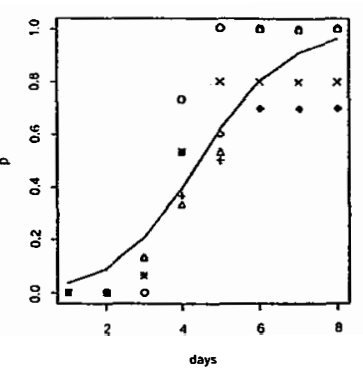

Isolado 741

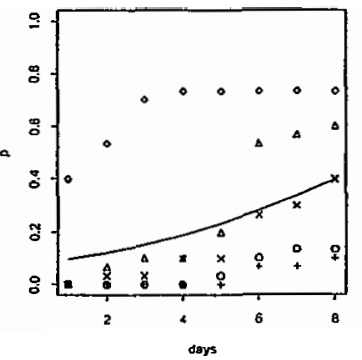

Isolado 843

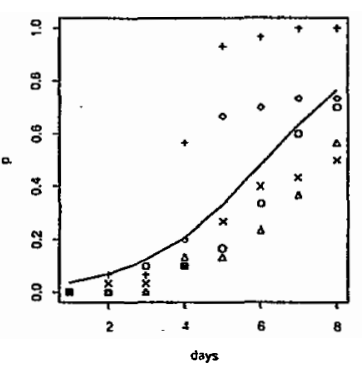

Isolado 744

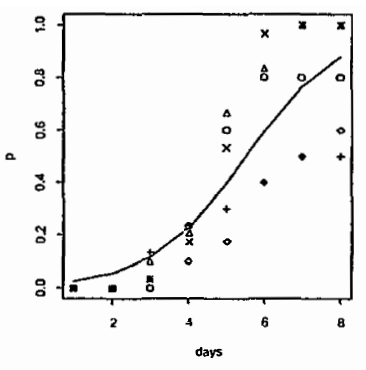

Isolado 752

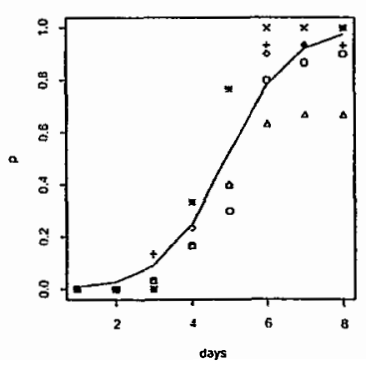

Isolado 573

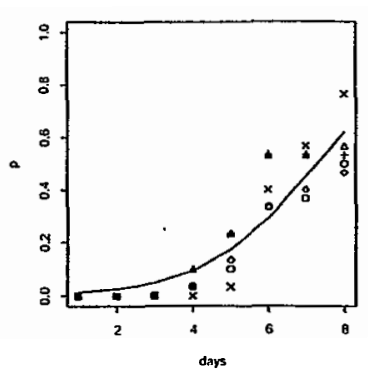

Isolado 784

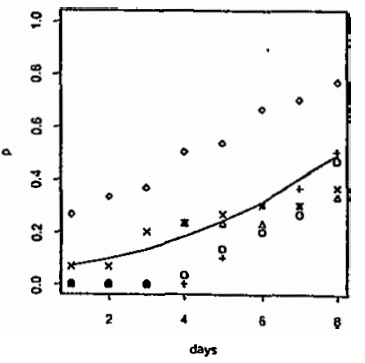

Isolado 845

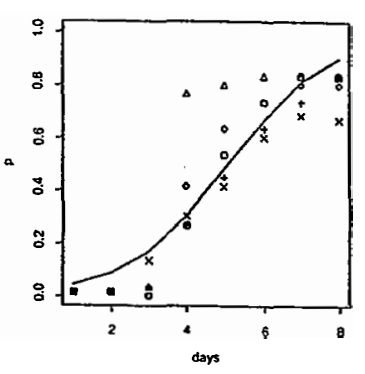

Isolado 747

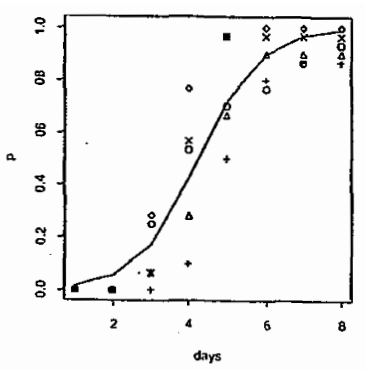

Isolado 755

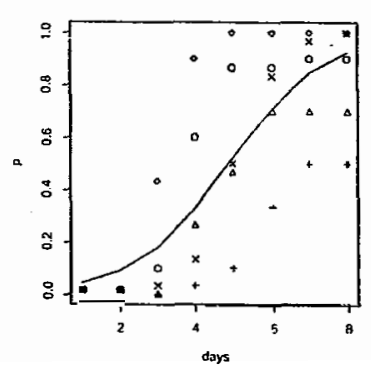

Isolado 591

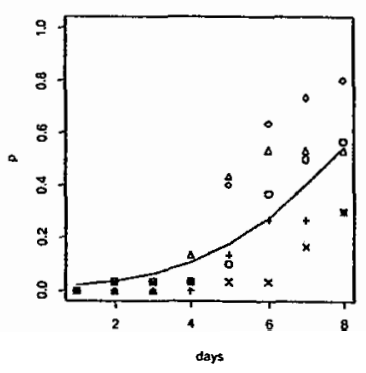

Figura 2: Proporção da mortalidade acumulada durante os 8 dias, para vinte isolados do experimento com o cupim $H$. tenuis. 

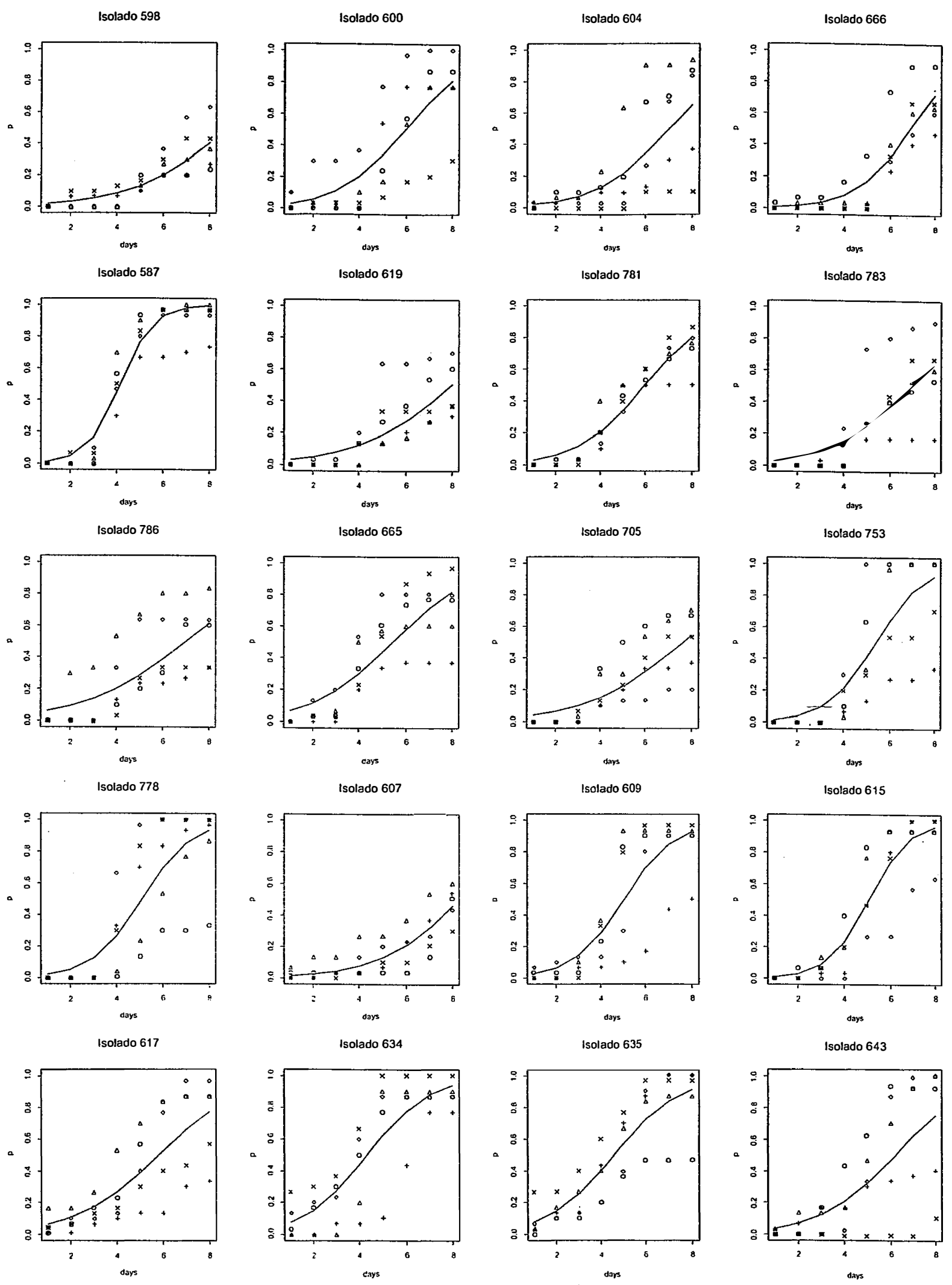

Figura 3: Proporção da mortalidade acumulada durante os 8 dias, para vinte isolados do experimento com o cupim $H$. tenuis. 
Isolado 651

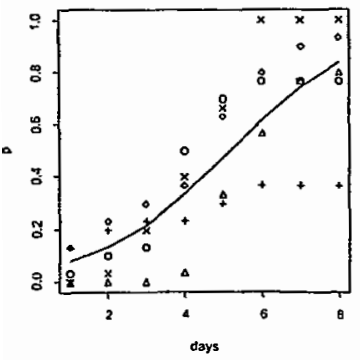

Isolado 509

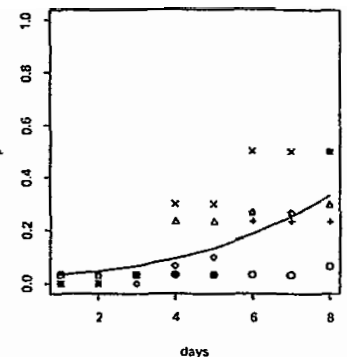

Isolado 535

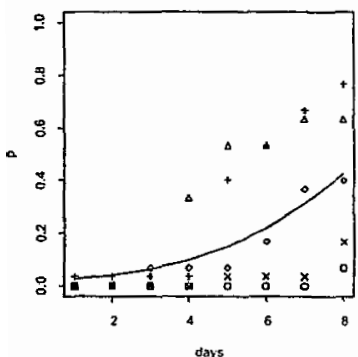

Isolado 560

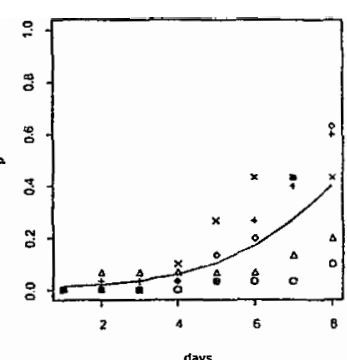

Isolado 555

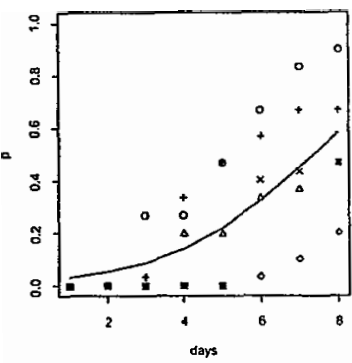

Isolado 286

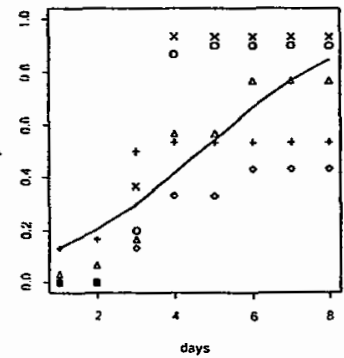

Isolado 515

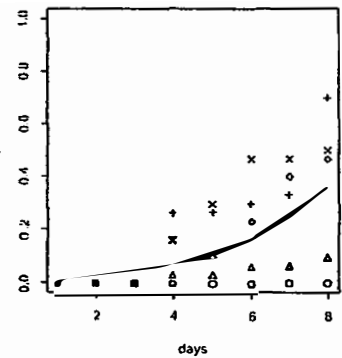

Isolado 545

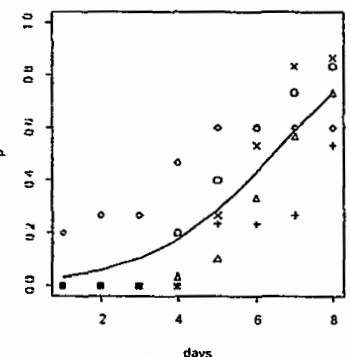

Isolado 585

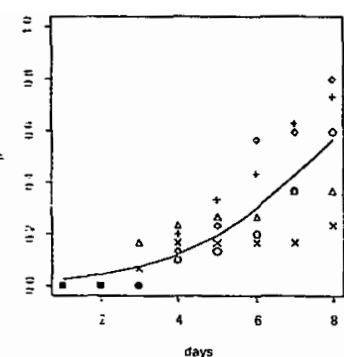

Isolado 558

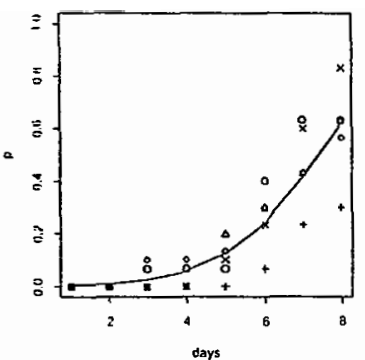

Isolado 565

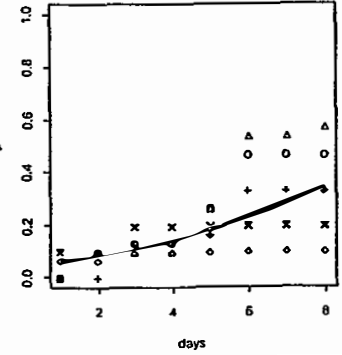

Isolado 520

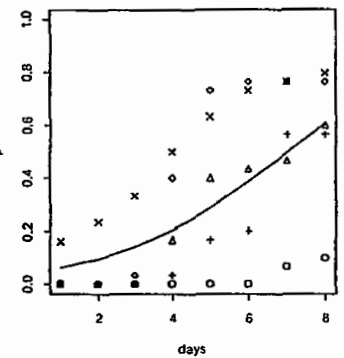

Isolado 553

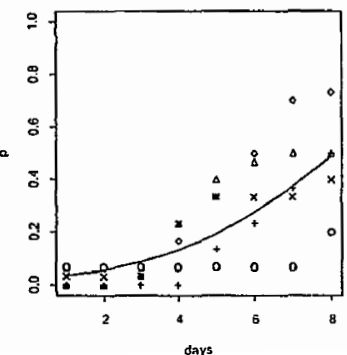

Isolado 532

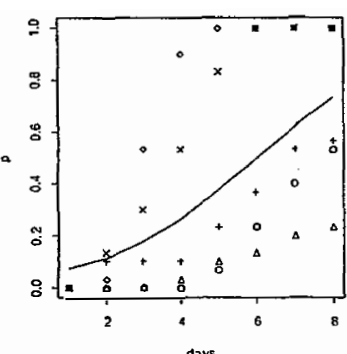

solado 562

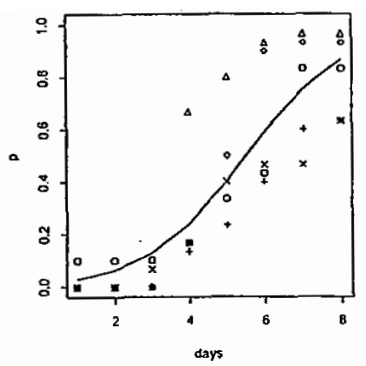

Isolado 571

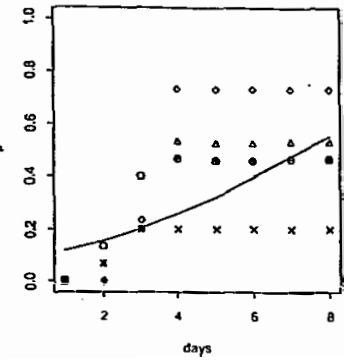

Isolado 531

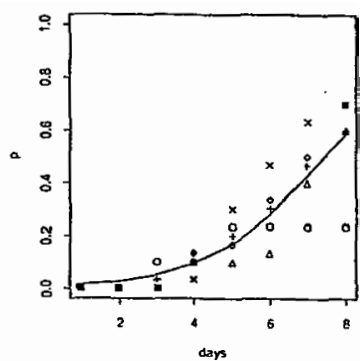

Isolado 556

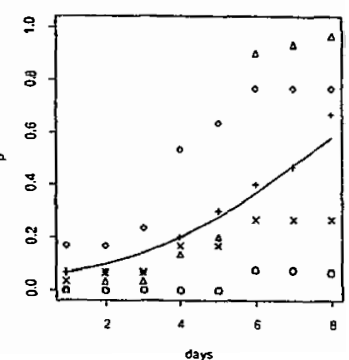

solado 554

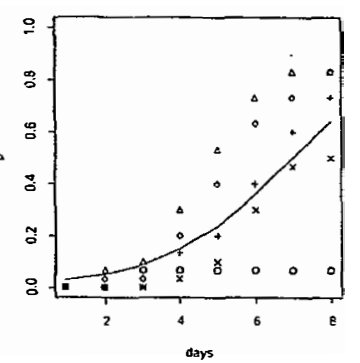

Isolado 566

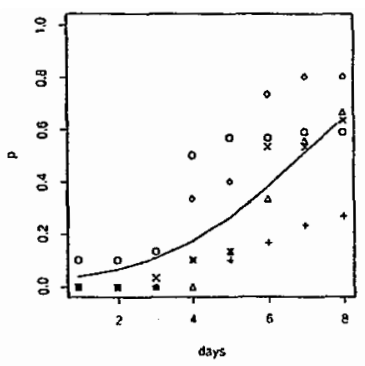

Figura 4: Proporção da mortalidade acumulada durante os 8 dias, para vinte isolados do experimento com o cupim $H$. tenuis. 
Isolado 569

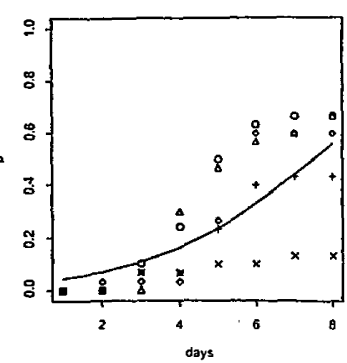

Isolado 534

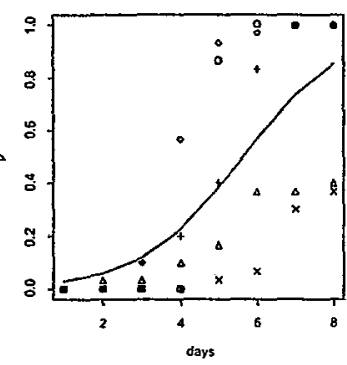

Isolado 544

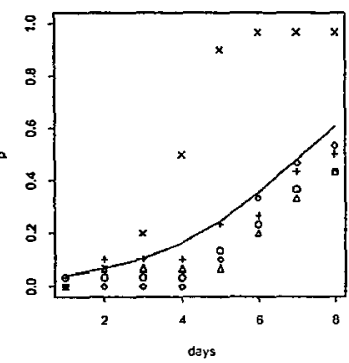

Isolado 391

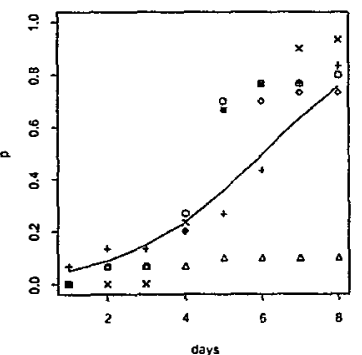

Isolado 498

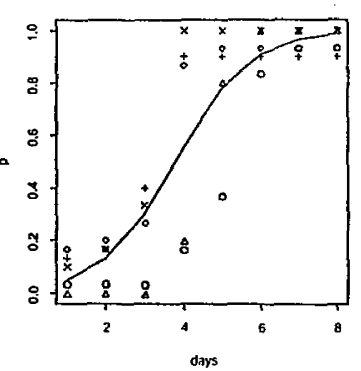

Isolado 572

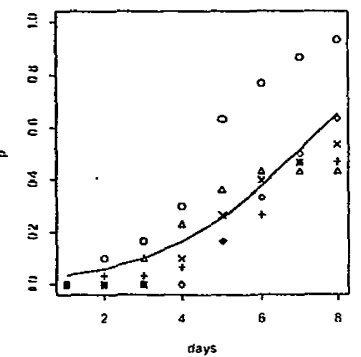

Isolado 536

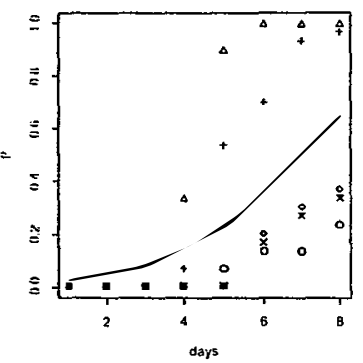

Isolado 548

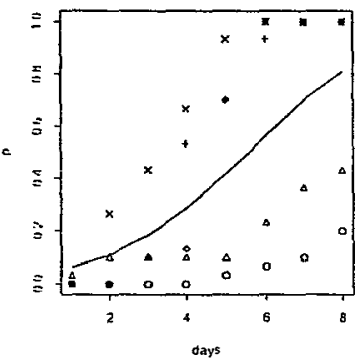

Isolado 484

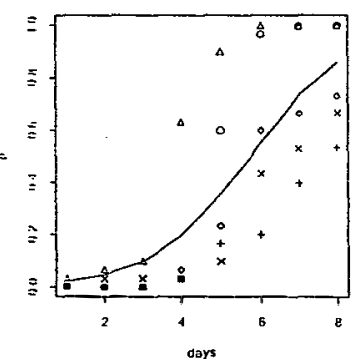

Isolado 512

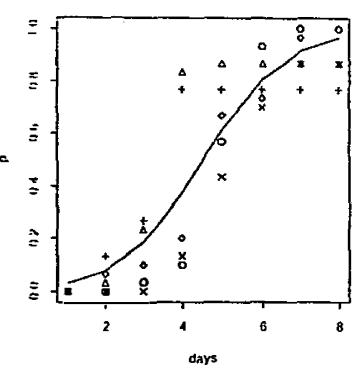

Isolado 574

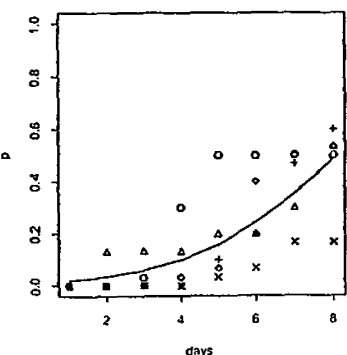

Isolado 538

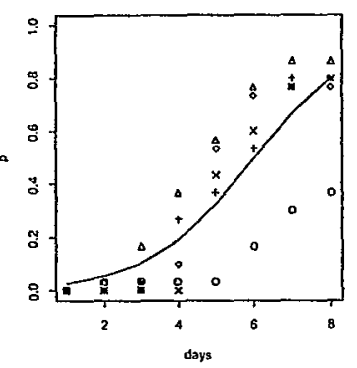

Isolado 549

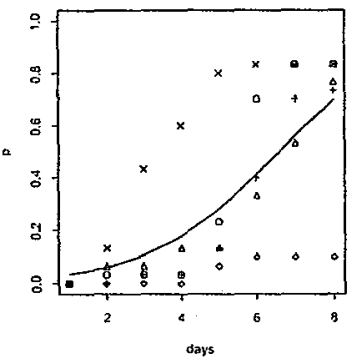

Isolado 485

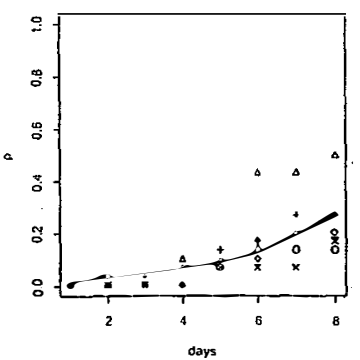

Isclado 524

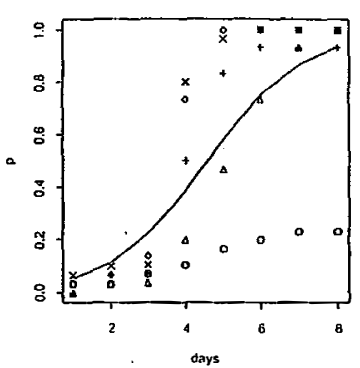

Isolado 448

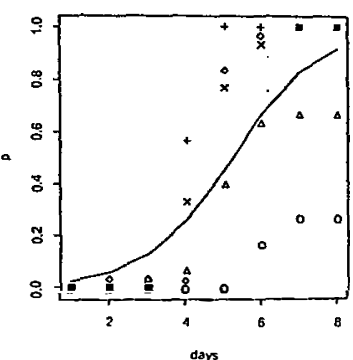

olado 542

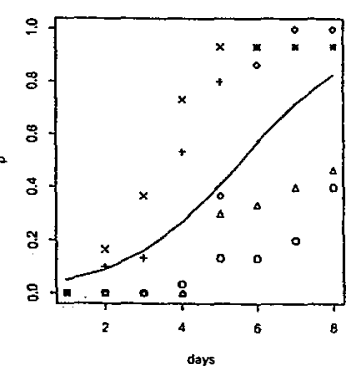

Isolado 550

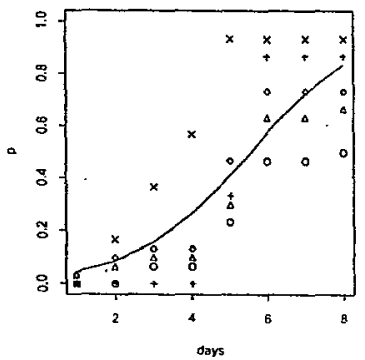

Isolado 489

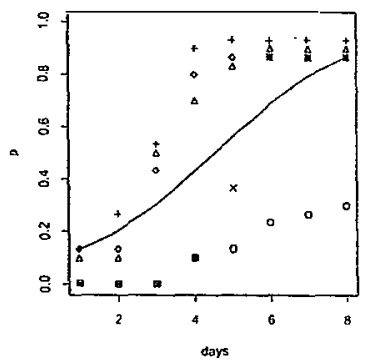

Isolado 529

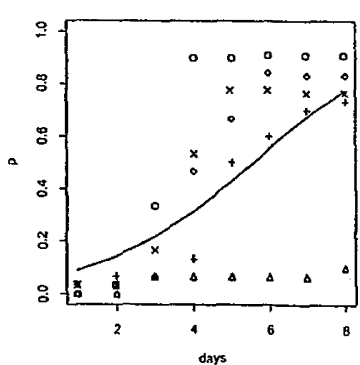

Figura 5: Proporção da mortalidade acumulada durante os 8 dias, para vinte isolados do experimento com o cupim H. tenuis. 

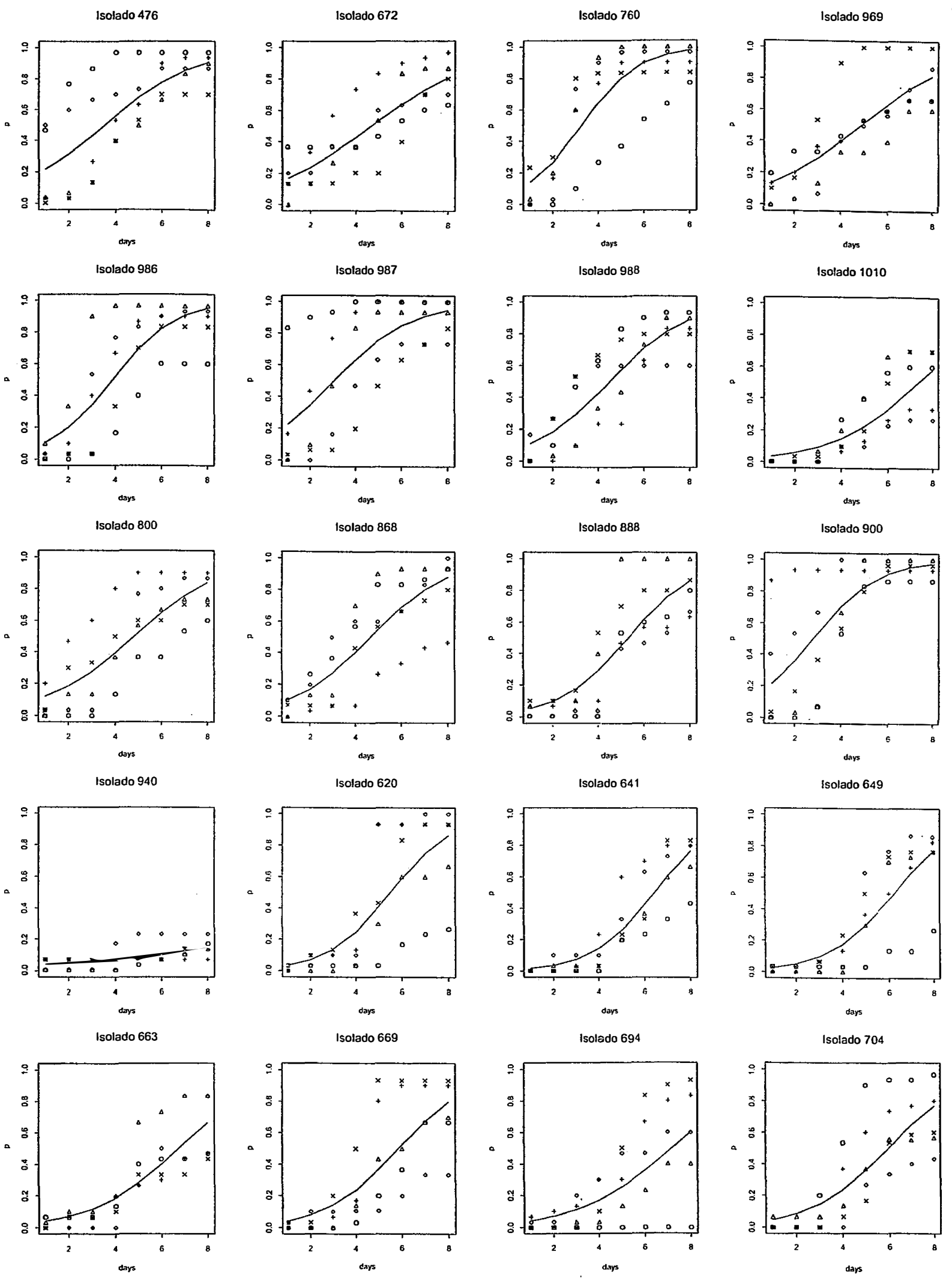

Figura 6: Proporção da mortalidade acumulada durante os 8 dias, para vinte isolados do experimento com o cupim H. tenuis. 

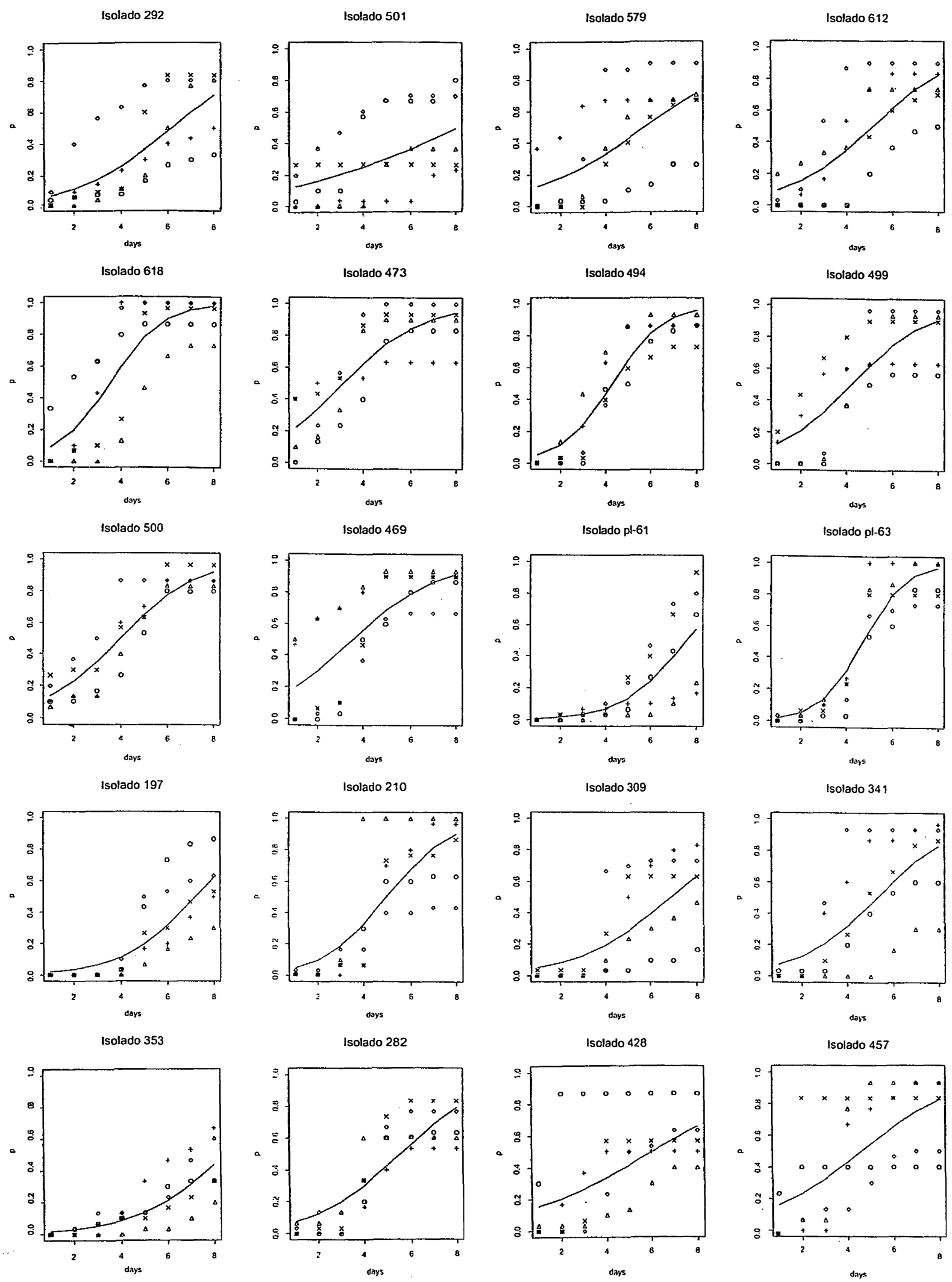

Figura 7: Proporção da mortalidade acumulada durante os 8 dias, para vinte isolados do experimento com o cupim H. tenuis. 

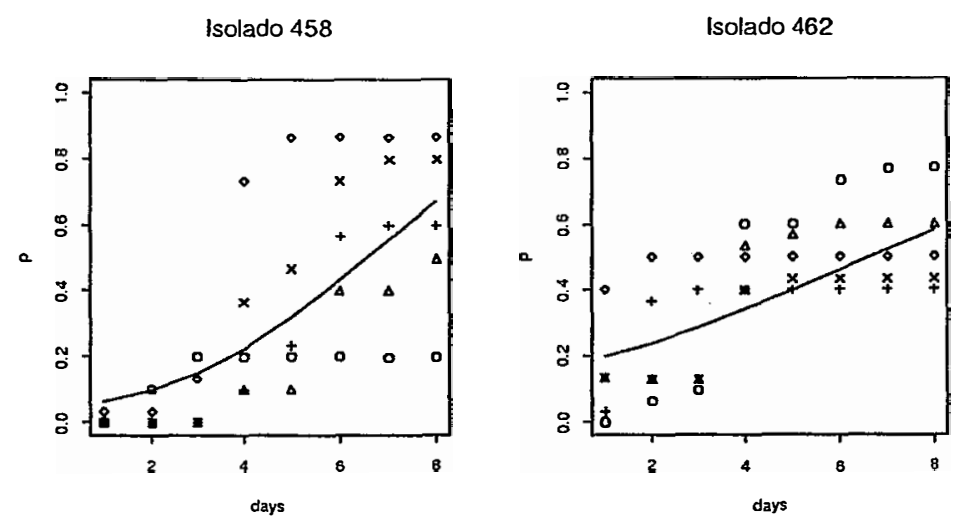

Figura 8: Proporção da mortalidade acumulada durante os 8 dias, para dois isolados do experimento com o cupim $H$. tenuis. 
(a) Assintótica
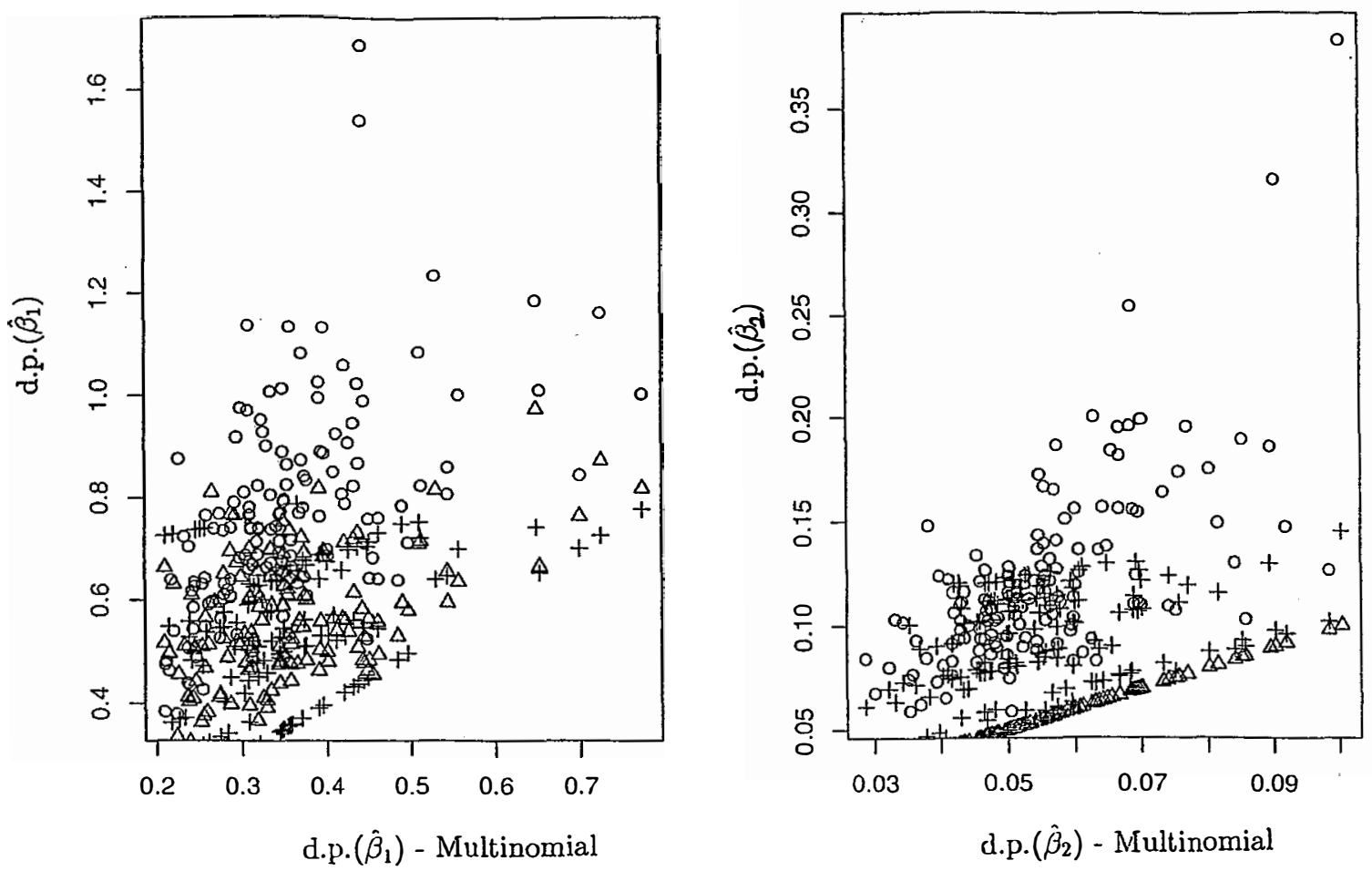

(b) Robusta
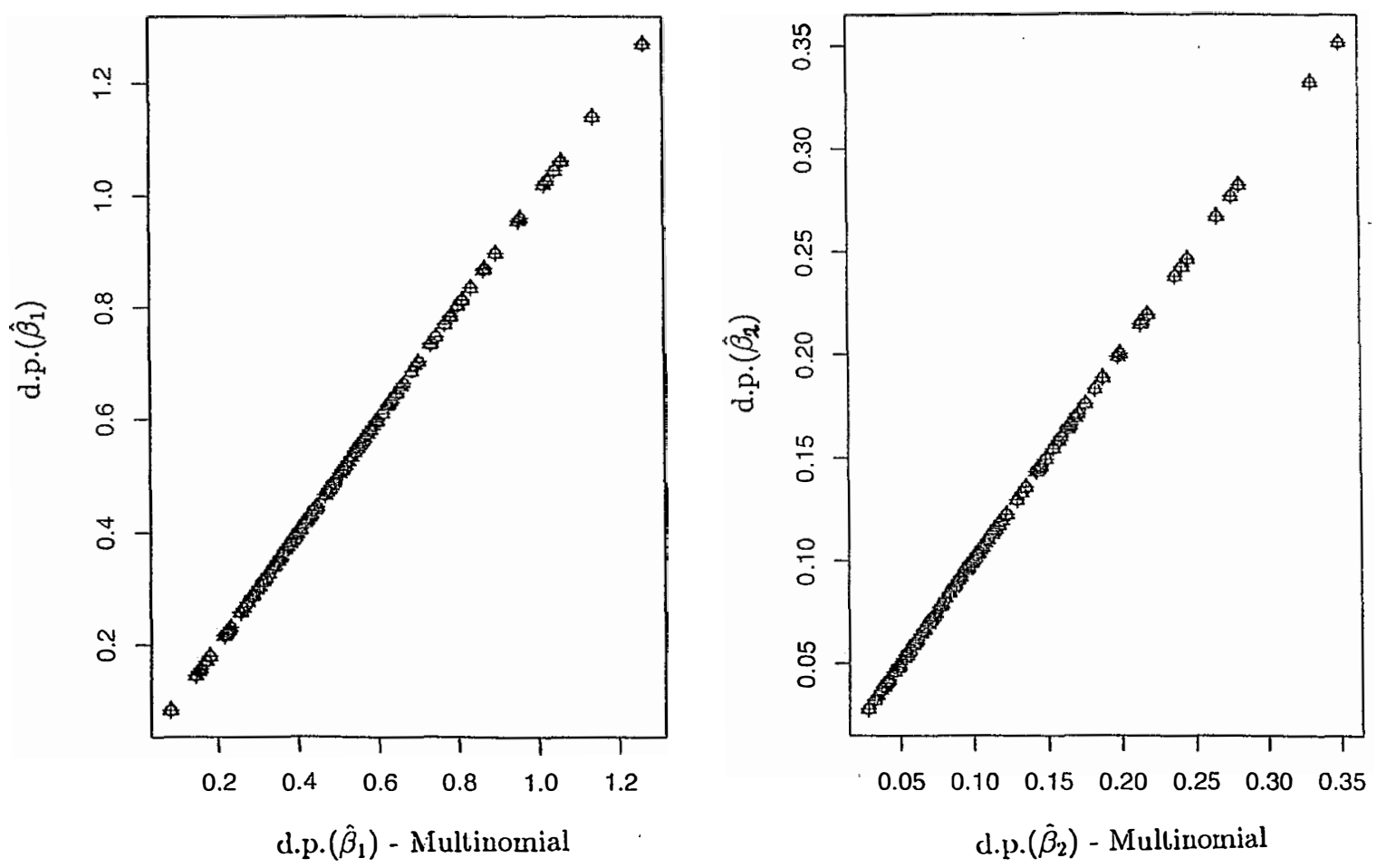

Figura 9: Diagramas de dispersão do d.p. $\left(\hat{\beta}_{1}\right)$ e d.p. $\left(\hat{\beta_{2}}\right)$ para o modelo multinomial $\times$ DM (o), $\operatorname{REMI}(\Delta)$ e REMII $(+)$, usando a matriz de variâncias e covariância assintótica (a) e robusta (b). 
(a)

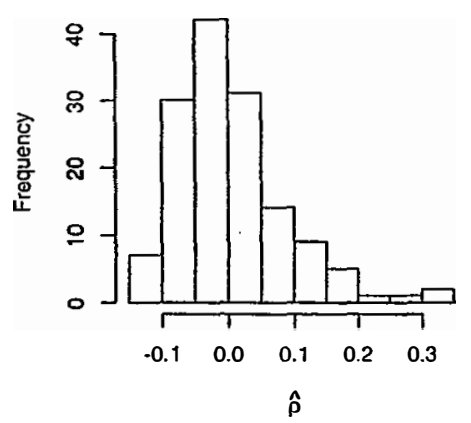

(d)

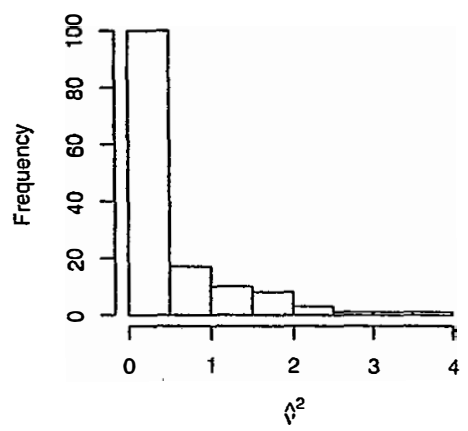

(g)

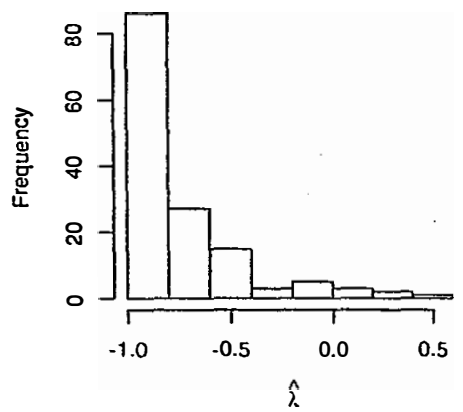

(j)

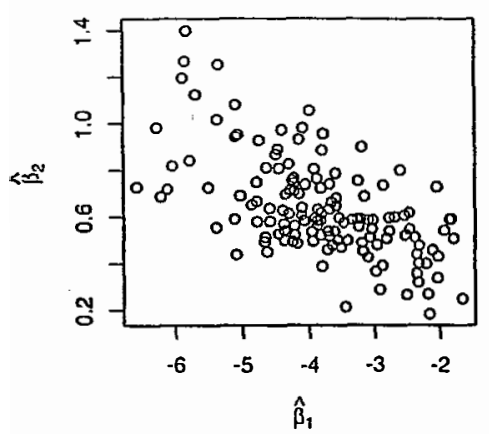

(b)

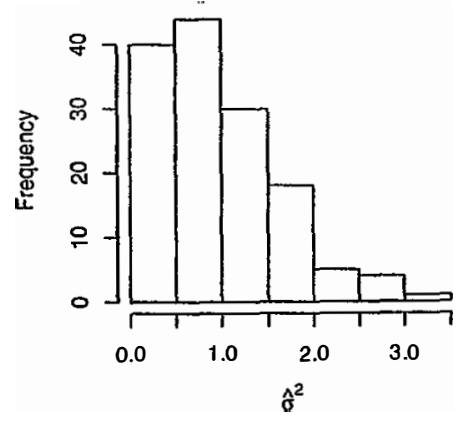

(e)

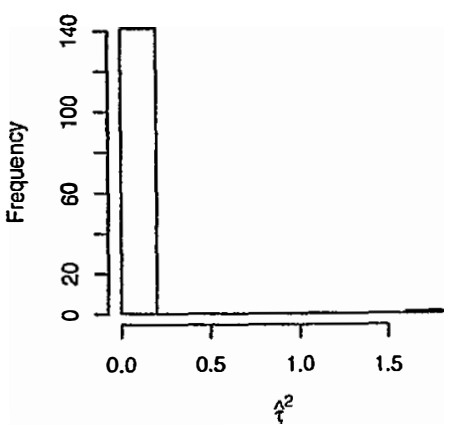

(h)

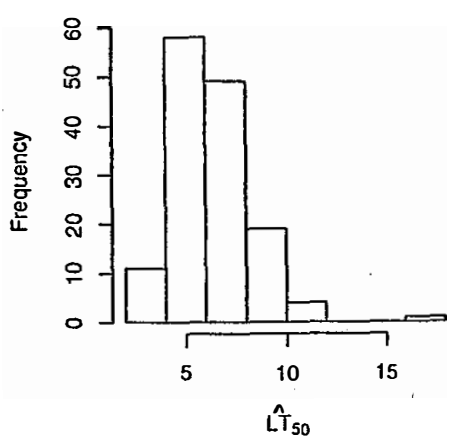

(k)

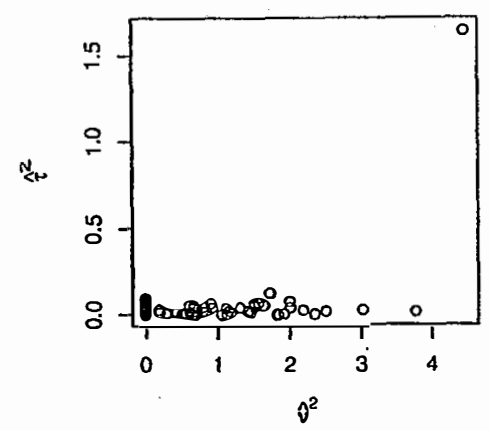

(c)

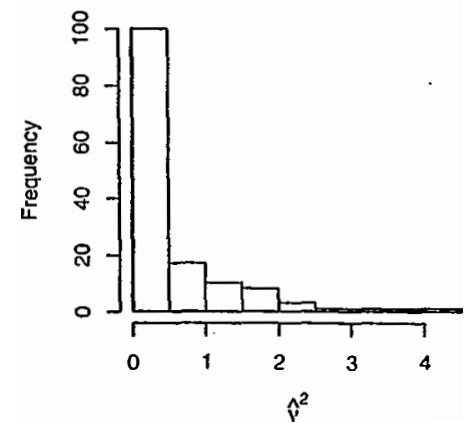

(f)

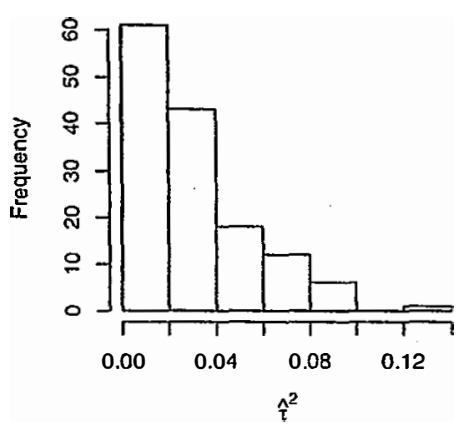

(i)

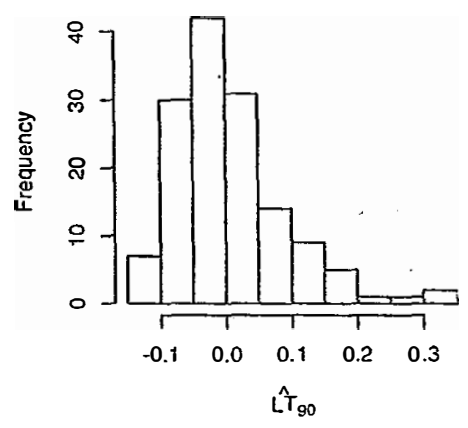

(1)

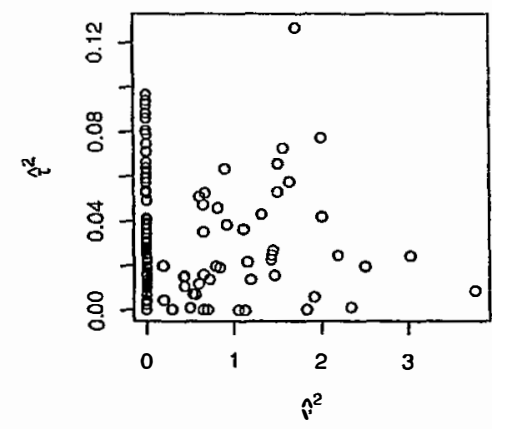

Figura 10: Histogramas de $\hat{\rho}, \hat{\sigma}^{2}, \hat{\nu}^{2}, \hat{\tau}^{2}\left(\operatorname{com}(c),(e)\right.$ e sem $(d),(f)$ o Isolado 987), $\hat{\lambda}, \hat{L T} T_{50}$ e $\hat{T}_{90}$ e os diagramas de dispersão do $\hat{\beta}_{1} \times \hat{\beta}_{2}$ e $\hat{\nu}^{2} \times \hat{\tau}^{2}$ (com $(k)$ e sem (l) o Isolado 987). 
Tabela 7: Estimativas dos desvios padrões (d.p.) das estimativas dos parâmetros do modelo de regressão calculadas através da matriz de variâncias e covariâncias assintótica, para o ensaio com os cupins.

\begin{tabular}{|c|c|c|c|c|c|c|c|c|c|}
\hline \multirow[t]{2}{*}{ Isolado } & \multirow[t]{2}{*}{$i$} & \multicolumn{2}{|c|}{ multinomial } & \multicolumn{2}{|c|}{ Dirichlet-multinomial } & \multicolumn{2}{|c|}{ REMI } & \multicolumn{2}{|c|}{ REMII } \\
\hline & & d.p. $\left(\hat{\beta}_{1}\right)$ & d.p. $\left(\hat{\beta}_{2}\right)$ & d.p. $\left(\hat{\beta}_{1}\right)$ & d.p. $\left(\hat{\beta}_{2}\right)$ & d.p. $\left(\hat{\beta}_{1}\right)$ & d.p. $\left(\hat{\beta}_{2}\right)$ & d.p. $\left(\hat{\beta}_{1}\right)$ & d.p. $\left(\hat{\beta}_{2}\right)$ \\
\hline 848 & 1 & 0,2424 & 0,0378 & 0,5449 & 0,085 & 0,2795 & 0,0378 & 0,2424 & 0,0471 \\
\hline 787 & 2 & 0,2982 & 0,0469 & 0,6829 & 0,1073 & 0,5137 & 0,0469 & 0,2982 & 0,0547 \\
\hline 1003 & 3 & 0,6524 & 0,0841 & 1,0087 & 0,1301 & 0,6652 & 0,0841 & 0,6524 & 0,0887 \\
\hline 1006 & 4 & 0,37 & 0,0526 & 0,8715 & 0,1239 & 0,6446 & 0,0526 & 0,3700 & 0,0597 \\
\hline 1024 & $\mathbf{5}$ & 0,6993 & 0,0857 & 0,8426 & 0,1032 & 0,762 & 0,0857 & 0,6993 & 0,0902 \\
\hline 852 & 6 & 0,2946 & 0,0397 & 0,917 & 0,1237 & 0,6737 & 0,0397 & 0,2946 & 0,0487 \\
\hline 879 & 7 & 0,7741 & 0,0984 & 1,0001 & 0,1271 & 0,8164 & 0,0984 & 0,7741 & 0,1023 \\
\hline 883 & 8 & 0,7243 & 0,092 & 1,1612 & 0,1476 & 0,87 & 0,092 & 0,7243 & 0,0962 \\
\hline 885 & 9 & 0,6484 & 0,0815 & 1,1878 & 0,1493 & 0,9713 & 0,0815 & 0,7404 & 0,1159 \\
\hline 957 & 10 & 0,4316 & 0,0597 & 0,821 & 0,1136 & 0,7171 & 0,0597 & 0,5604 & 0,1017 \\
\hline 1028 & 11 & 0,3262 & 0,0701 & 0,927 & 0,1993 & 0,4111 & 0,0701 & 0,4839 & 0,1082 \\
\hline 732 & 12 & 0,3183 & 0,054 & 0,7142 & 0,1212 & 0,6143 & 0,054 & 0,4786 & 0,0985 \\
\hline 743 & 13 & 0,3925 & 0,0688 & 0,8881 & 0,1557 & 0,5584 & 0,0688 & 0,5309 & 0,1073 \\
\hline 745 & 14 & 0,5286 & 0,0667 & 1,237 & 0,1562 & 0,8129 & 0,0667 & 0,6381 & 0,1060 \\
\hline 767 & 15 & 0,3496 & 0,0502 & 0,7952 & 0,1142 & 0,689 & 0,0502 & 0,5000 & 0,0965 \\
\hline 823 & 16 & 0,3443 & 0,0498 & 0,6584 & 0,0953 & 0,4761 & 0,0498 & 0,4963 & 0,0963 \\
\hline 855 & 17 & 0,4208 & 0,0554 & 10.589 & 0,1394 & 0,7104 & 0,0554 & 0,4208 & 0,0595 \\
\hline 857 & 18 & 0,4488 & 0,0595 & 0,7562 & 0,1003 & 0,563 & 0,0595 & 0,4488 & 0,0634 \\
\hline 876 & 19 & 0,4962 & 0,0751 & 0,7102 & 0,1075 & 0,5796 & 0,0751 & 0,4962 & 0,0782 \\
\hline 878 & 20 & 0,4438 & 0,0573 & 0,7085 & 0,0915 & 0,4779 & 0,0573 & 0,4438 & 0,0613 \\
\hline 602 & 21 & 0,2366 & 0,0358 & 0,5083 & 0,077 & 0,4126 & 0,0358 & 0,2366 & 0,0419 \\
\hline 616 & 22 . & 0,2645 & 0,0301 & 0,5977 & 0,068 & 0,8105 & 0,0301 & 0,2645 & 0,0371 \\
\hline 741 & 23 & 0,2867 & 0,0352 & 0,6085 & 0,0747 & 0,6954 & 0,0352 & 0,2867 & 0,0414 \\
\hline 784 & 24 & 0,3123 & 0,0407 & 0,5023 & 0,0655 & 0,4702 & 0,0407 & 0,3123 & 0,0462 \\
\hline 822 & 25 & 0,254 & 0,0353 & 0,4273 & 0,0593 & 0,3749 & 0,0353 & 0,4730 & 0,1006 \\
\hline 841 & 26 & 0,3483 & 0,0563 & 0,6764 & 0,1094 & 0,4955 & 0,0563 & 0,5296 & 0,1098 \\
\hline 843 & 27 & 0,3356 & 0,0509 & $\mathbf{0 , 7 3 7 8}$ & 0,1118 & 0,5898 & 0,0509 & 0,5214 & 0,1071 \\
\hline 845 & 28 & 0,3192 & 0,0562 & 0,689 & 0,1214 & 0,3664 & 0,0562 & 0,5110 & 0,1097 \\
\hline 738 & 29 & 0,3454 & 0,0605 & 0,7147 & 0,1253 & 0,4398 & 0,0605 & $\mathbf{0 , 5 2 7 8}$ & 0,1120 \\
\hline 742 & 30 & 0,3483 & 0,0502 & 0,8885 & 0,1281 & 0,5893 & 0,0502 & 0,5297 & 0,1068 \\
\hline 744 & 31 & 0,3587 & 0,0599 & 0,7156 & 0,1194 & 0,5145 & 0,0599 & 0,5365 & 0,1116 \\
\hline 747 & 32 & 0,4077 & 0,0895 & 0,8491 & 0,1864 & 0,564 & 0,0895 & 0,5705 & 0,1299 \\
\hline 748 & 33 & 0,3078 & 0,0686 & 11.372 & 0,2537 & 0,4657 & 0,0686 & 0,4440 & 0,0783 \\
\hline 750 & 34 & 0,4115 & 0,0732 & 0,9231 & 0,1641 & 0,5403 & 0,0732 & 0,5213 & 0,0823 \\
\hline 752 & 35 & 0,4436 & 0,0852 & 0,9877 & 0,1897 & 0,5292 & 0,0852 & 0,5470 & 0,0932 \\
\hline 755 & 36 & 0,3196 & 0,0586 & 0,823 & 0,151 & 0,6567 & 0,0586 & 0,4523 & 0,0697 \\
\hline
\end{tabular}


Continuação da Tabela (7). Estimativas dos desvios padrões (d.p.) das estimativas dos parâmetros do modelo de regressão calculadas através da matriz de variâncias e covariâncias assintótica, para o ensaio com os cupins.

\begin{tabular}{|c|c|c|c|c|c|c|c|c|c|}
\hline \multirow[t]{2}{*}{ Isolado } & \multirow[t]{2}{*}{$i$} & \multicolumn{2}{|c|}{ multinomial } & \multicolumn{2}{|c|}{ Dirichlet-multinomial } & \multicolumn{2}{|c|}{ REMI } & \multicolumn{2}{|c|}{ REMII } \\
\hline & & d.p. $\left(\hat{\beta}_{1}\right)$ & d.p. $\left(\hat{\beta}_{2}\right)$ & d.p. $\left(\hat{\beta}_{1}\right)$ & d.p. $\left(\hat{\beta}_{2}\right)$ & d.p. $\left(\hat{\beta}_{1}\right)$ & d.p. $\left(\hat{\beta}_{2}\right)$ & d.p. $\left(\hat{\beta}_{1}\right)$ & d.p. $\left(\hat{\beta}_{2}\right)$ \\
\hline 756 & 37 & 0,4414 & 0,0903 & 15.432 & 0,3159 & 0,5824 & 0,0903 & 0,5452 & 0,0979 \\
\hline 764 & 38 & 0,3302 & 0,0667 & 0,9001 & 0,1819 & 0,3919 & 0,0667 & 0,4599 & 0,0766 \\
\hline 573 & 39 & 0,4545 & 0,0625 & 0,6805 & 0,0936 & 0,4548 & 0,0625 & 0,5559 & 0,0730 \\
\hline 591 & 40 & 0,4219 & 0,0566 & 0,7865 & 0,1055 & 0,5604 & 0,0566 & 0,5296 & 0,0679 \\
\hline 598 & 41 & 0,461 & 0,0593 & 0,7582 & 0,0975 & 0,4945 & 0,0593 & 0,7284 & 0,1210 \\
\hline 600 & 42 & 0,3536 & 0,0551 & 0,8233 & 0,1283 & 0,6207 & 0,0551 & 0,6657 & 0,1191 \\
\hline 604 & 43 & 0,3959 & 0,0555 & 0,8853 & 0,1241 & 0,6938 & 0,0555 & 0,6891 & 0,1192 \\
\hline 666 & 44 & 0,4889 & 0,0696 & 0,7817 & 0,1113 & 0,5941 & 0,0696 & 0,7464 & 0,1264 \\
\hline 587 & 45 & 0,4424 & 0,1004 & 16.894 & 0,3835 & 0,4838 & 0,1004 & 0,7168 & 0,1457 \\
\hline 619 & 46 & 0,3916 & 0,0518 & 0,7617 & 0,1008 & 0,5039 & 0,0518 & 0,6866 & 0,1176 \\
\hline 781 & 47 & 0,3487 & 0,0543 & 0,5689 & 0,0886 & 0,3487 & 0,0543 & 0,6631 & 0,1187 \\
\hline 783 & 48 & 0,3722 & 0,0521 & 0,7784 & 0,1089 & 0,5475 & 0,0521 & 0,6757 & 0,1177 \\
\hline 786 & 49 & 0,3115 & 0,0431 & 0,7412 & 0,1025 & 0,508 & 0,0431 & 0,3115 & 0,0559 \\
\hline 665 & 50 & 0,2872 & 0,0465 & 0,6409 & 0,1038 & 0,3985 & 0,0465 & 0,2872 & 0,0586 \\
\hline 705 & 51 & 0,3584 & 0,0481 & 0,685 & 0,092 & 0,4751 & 0,0481 & 0,3584 & 0,0598 \\
\hline 753 & 52 & 0,3957 & 0,0684 & 11.349 & 0,1962 & 0,6832 & 0,0684 & 0,3957 & 0,0771 \\
\hline 778 & 53 & 0,3705 & 0,0668 & 10.823 & 0,1953 & 0,72 & 0,0668 & 0,3705 & 0,0757 \\
\hline 607 & 54 & 0,4839 & 0,0632 & 0,6369 & 0,0832 & 0,5295 & 0,0632 & 0,4839 & 0,0726 \\
\hline 609 & 55 & 0,3536 & 0,0643 & 0,8625 & 0,1568 & 0,6088 & 0,0643 & 0,3536 & 0,0735 \\
\hline 615 & 56 & 0,4316 & 0,0802 & 0,944 & 0,1754 & 0,6128 & 0,0802 & 0,4316 & 0,0877 \\
\hline 617 & 57 & 0,294 & 0,0454 & 0,5347 & 0,0826 & 0,5202 & 0,0454 & 0,5569 & 0,0790 \\
\hline 634 & 58 & 0,2786 & 0,0553 & 0,6117 & 0,1214 & 0,5262 & 0,0553 & 0,5489 & 0,0850 \\
\hline 635 & 59 & $0 ; 2747$ & 0,0512 & 0,5648 & 0,1053 & 0,4178 & 0,0512 & 0,5469 & 0,0825 \\
\hline 643 & 60 & 0,3347 & 0,0503 & 0,8046 & 0,1208 & 0,6984 & 0,0503 & 0,5794 & 0,0819 \\
\hline 651 & 61 & 0,2738 & 0,0458 & 0,5281 & 0,0883 & 0,4123 & 0,0458 & 0,5465 & 0,0792 \\
\hline 268 & 62 & 0,2381 & 0,0412 & 0,7051 & 0,122 & 0,4041 & 0,0412 & 0,5295 & 0,0766 \\
\hline 565 & 63 & 0,3302 & 0,0404 & 0,6639 & 0,0812 & 0,4067 & 0,0404 & 0,5768 & 0,0762 \\
\hline 571 & 64 & 0,2582 & 0,0343 & 0,7654 & 0,1018 & 0,3823 & 0,0343 & 0,5388 & 0,0732 \\
\hline 509 & 65 & 0,4259 & 0,0532 & 0,9054 & 0,1131 & 0,5386 & 0,0532 & 0,6960 & 0,1243 \\
\hline 515 & 66 & 0,5096 & 0,0649 & 10.836 & 0,138 & 0,7083 & 0,0649 & 0,7502 & 0,1297 \\
\hline 520 & 67 & 0,3095 & 0,0428 & 0,676 & 0,0935 & 0,6039 & 0,0428 & 0,6316 & 0,1202 \\
\hline 531 & 68 & 0,4496 & 0,0611 & 0,6408 & 0,0871 & 0,4834 & 0,0611 & 0,7108 & 0,1279 \\
\hline 535 & 69 & 0,4363 & 0,0563 & 10.221 & 0,132 & 0,729 & 0,0563 & 0,7024 & 0,1257 \\
\hline 545 & 70 & 0,358 & 0,0527 & 0,6408 & 0,0944 & 0,4448 & 0,0527 & 0,6567 & 0,1241 \\
\hline 553 & 71 & 0,3762 & 0,0494 & 0,6079 & 0,0799 & 0,4836 & 0,0494 & 0,6667 & 0,1227 \\
\hline 556 & 72 & 0,3094 & 0,0421 & 0,7808 & 0,1063 & 0,6062 & 0,0421 & 0,6315 & 0,1200 \\
\hline
\end{tabular}


Continuação da Tabela (7). Estimativas dos desvios padrões (d.p.) das estimativas dos parâmetros do modelo de regressão calculadas através da matriz de variâncias e covariâncias assintótica, para o ensaio com os cupins.

\begin{tabular}{|c|c|c|c|c|c|c|c|c|c|}
\hline \multirow[t]{2}{*}{ Isolado } & \multirow[t]{2}{*}{$i$} & \multicolumn{2}{|c|}{ multinomial } & \multicolumn{2}{|c|}{ Dirichlet-multinomial } & \multicolumn{2}{|c|}{ REMI } & \multicolumn{2}{|c|}{ REMII } \\
\hline & & d.p. $\left(\hat{\beta}_{1}\right)$ & d.p. $\left(\hat{\beta}_{2}\right)$ & d.p. $\left(\hat{\beta}_{1}\right)$ & d.p. $\left(\hat{\beta}_{2}\right)$ & d.p. $\left(\hat{\beta}_{1}\right)$ & d.p. $\left(\hat{\beta}_{2}\right)$ & d.p. $\left(\hat{\beta}_{1}\right)$ & d.p. $\left(\hat{\beta}_{2}\right)$ \\
\hline 560 & 73 & 0,5431 & 0,0699 & 0,857 & 0,1104 & 0,6541 & 0,0699 & 0,6446 & 0,1214 \\
\hline 585 & 74 & 0,4013 & 0,0543 & 0,685 & 0,0927 & 0,4807 & 0,0543 & $\mathbf{0 , 5 3 0 7}$ & 0,1132 \\
\hline 532 & 75 & 0,2873 & 0,0432 & 0,7417 & 0,1115 & 0,7677 & 0,0432 & 0,4507 & 0,1083 \\
\hline 554 & 76 & 0,3736 & 0,0523 & 0,6438 & 0,0902 & 0,6077 & 0,0523 & 0,5100 & 0,1122 \\
\hline 555 & 77 & 0,3758 & 0,0512 & 0,8323 & 0,1135 & 0,601 & 0,0512 & 0,5117 & 0,1117 \\
\hline 558 & 78 & 0,5429 & 0,074 & 0,8041 & 0,1096 & 0,5944 & 0,074 & 0,6445 & 0,1238 \\
\hline 562 & 79 & 0,3451 & 0,0573 & 0,7665 & 0,1272 & 0,512 & 0,0573 & 0,4896 & 0,1146 \\
\hline 566 & 80 & 0,3455 & 0,0485 & 0,7378 & 0,1037 & 0,4759 & 0,0485 & 0,4899 & 0,1105 \\
\hline 569 & 81 & 0,3444 & 0,0466 & 0,6915 & 0,0935 & 0,4811 & 0,0466 & 0,3444 & 0,0822 \\
\hline 572 & 82 & 0,3559 & 0,0502 & 0,5323 & 0,0751 & 0,479 & 0,0502 & 0,3559 & 0,0843 \\
\hline 574 & 83 & 0,4367 & 0,0575 & 0,8652 & 0,1139 & 0,5077 & 0,0575 & 0,4367 & 0,0889 \\
\hline 448 & 84 & 0,3567 & 0,063 & 11.355 & 0,2007 & 0,7339 & 0,063 & 0,3567 & 0,0926 \\
\hline 534 & 85 & 0,3487 & 0,0569 & 10.131 & 0,1654 & 0,752 & 0,0569 & 0,3487 & 0,0885 \\
\hline 536 & 86 & 0,3907 & 0,0546 & 10.251 & 0,1433 & 0,8165 & 0,0546 & 0,3907 & 0,0871 \\
\hline 538 & 87 & 0,3589 & 0,0556 & 0,6636 & 0,1028 & 0,525 & 0,0556 & 0,3589 & 0,0877 \\
\hline 542 & 88 & 0,3093 & 0,05 & 0,7658 & 0,1238 & 0,6998 & 0,05 & 0,3093 & 0,0842 \\
\hline 544 & 89 & 0,3499 & 0,0483 & 0,6522 & 0,09 & 0,627 & 0,0483 & 0,5460 & 0,1208 \\
\hline 548 & 90 & 0,2916 & 0,0467 & 0,7906 & 0,1265 & 0,7644 & 0,0467 & 0,5106 & 0,1201 \\
\hline 549 & 91 & 0,35 & 0,0506 & 0,7904 & 0,1144 & 0,6289 & 0,0506 & 0,5460 & 0,1217 \\
\hline 550 & 92 & 0,3129 & 0,0509 & 0,6684 & 0,1088 & 0,4913 & 0,0509 & $\mathbf{0 , 5 2 3 0}$ & 0,1218 \\
\hline 391 & 93 & 0,3112 & 0,0472 & 0,7385 & 0,112 & 0,5375 & 0,0472 & 0,5221 & 0,1203 \\
\hline 484 & 94 & 0,3736 & 0,0606 & 0,84 & 0,1363 & 0,6905 & 0,0606 & 0,5615 & 0,1262 \\
\hline 485 & 95 & 0,5564 & 0,0692 & 0,9988 & 0,1242 & 0,6338 & 0,0692 & 0,6966 & 0,1305 \\
\hline 489 & 96 & 0,2411 & 0,043 & 0,618 & 0,1103 & 0,6102 & 0,043 & 0,4835 & 0,1187 \\
\hline 498 & 97 & 0,3205 & 0,0756 & 0,7375 & 0,1739 & 0,6046 & 0,0756 & 0,3205 & 0,1111 \\
\hline 512 & 98 & 0,3464 & 0,0695 & 0,7699 & 0,1545 & 0,4811 & 0,0695 & 0,3464 & 0,1070 \\
\hline 524 & 99 & 0,2992 & 0,0573 & 0,9745 & 0,1867 & 0,645 & 0,0573 & 0,2992 & 0,0995 \\
\hline 529 & 100 & 0,2674 & 0,0419 & 0,7398 & 0,1159 & 0,596 & 0,0419 & 0,2674 & 0,0915 \\
\hline 476 & 101 & 0,2093 & 0,0418 & 0,3836 & 0,0765 & 0,5176 & 0,0418 & 0,2093 & 0,0915 \\
\hline 672 & 102 & 0,2231 & 0,0369 & 0,3791 & 0,0627 & 0,3346 & 0,0369 & 0,2231 & 0,0893 \\
\hline 760 & 103 & 0,2442 & 0,06 & 0,6352 & 0,156 & 0,5045 & 0,06 & 0,2442 & 0,1011 \\
\hline 969 & 104 & 0,2365 & 0,0394 & 0,4395 & 0,0732 & 0,413 & 0,0394 & 0,2365 & 0,0904 \\
\hline 986 & 105 & 0,2568 & 0,0545 & 0,6434 & 0,1366 & 0,5673 & 0,0545 & 0,7408 & 0,0828 \\
\hline 987 & 106 & 0,2104 & 0,0477 & 0,4765 & 0,108 & 0,6643 & 0,0477 & 0,7261 & 0,0785 \\
\hline 988 & 107 & 0,2513 & 0,046 & 0,5129 & 0,094 & 0,3627 & 0,046 & 0,7389 & 0,0775 \\
\hline 1010 & 108 & 0,3654 & 0,0499 & 0,6319 & 0,0862 & 0,4769 & 0,0499 & 0,7851 & 0,0798 \\
\hline 800 & 109 & 0,246 & 0,0418 & 0,4896 & 0,0832 & 0,443 & 0,0418 & 0,7372 & 0,0750 \\
\hline
\end{tabular}


Continuação da Tabela (7). Estimativas dos desvios padrões (d.p.) das estimativas dos parâmetros do modelo de regressão calculadas através da matriz de variâncias e covariâncias assintótica, para o ensaio com os cupins.

\begin{tabular}{|c|c|c|c|c|c|c|c|c|c|}
\hline \multirow[t]{2}{*}{ Isolado } & & \multicolumn{2}{|c|}{ multinomial } & \multicolumn{2}{|c|}{ Dirichlet-multinomial } & \multicolumn{2}{|c|}{ REMI } & \multicolumn{2}{|c|}{ REMII } \\
\hline & & d.p. $\left(\hat{\beta}_{1}\right)$ & d.p. $\left(\hat{\beta}_{2}\right)$ & d.p. $\left(\hat{\beta}_{1}\right)$ & d.p. $\left(\hat{\beta}_{2}\right)$ & d.p. $\left(\hat{\beta}_{1}\right)$ & d.p. $\left(\hat{\beta}_{2}\right)$ & d.p. $\left(\hat{\beta}_{1}\right)$ & d.p. $\left(\hat{\beta}_{2}\right)$ \\
\hline 868 & 110 & 0,2564 & 0,0459 & 0,5503 & 0,0985 & 0,4692 & 0,0459 & 0,7407 & 0,0774 \\
\hline 888 & 111 & 0,305 & 0,0514 & 0,6884 & 0,1161 & 0,4807 & 0,0514 & 0,7589 & 0,0808 \\
\hline 900 & 112 & 0,2199 & 0,0573 & 0,5411 & 0,1409 & 0,6315 & 0,0573 & 0,7289 & 0,0846 \\
\hline 940 & 113 & 0,4475 & 0,0504 & 0,5241 & 0,0591 & 0,463 & 0,0504 & 0,7024 & 0,1131 \\
\hline 620 & 114 & 0,3347 & 0,0554 & 10.069 & 0,1668 & 0,6464 & 0,0554 & 0,6364 & 0,1154 \\
\hline 641 & 115 & 0,4005 & 0,0597 & 0,6974 & 0,1039 & 0,4995 & 0,0597 & 0,6734 & 0,1175 \\
\hline 649 & 116 & 0,3679 & 0,0555 & 0,7685 & 0,116 & 0,5596 & 0,0555 & 0,6545 & 0,1155 \\
\hline 663 & 117 & 0,335 & 0,0476 & 0,6722 & 0,0956 & 0,4254 & 0,0476 & 0,6366 & 0,1119 \\
\hline 669 & 118 & 0,3202 & 0,05 & 0,7408 & 0,1157 & 0,5879 & 0,05 & 0,6289 & 0,1129 \\
\hline 694 & 119 & 0,3414 & 0,0471 & 0,7439 & 0,1025 & 0,644 & 0,0471 & 0,6400 & 0,1116 \\
\hline 704 & 120 & 0,3141 & 0,0481 & 0,6694 & 0,1025 & 0,521 & 0,0481 & 0,6259 & 0,1121 \\
\hline 292 & 121 & 0,2831 & 0,0418 & 0,6208 & 0,0916 & 0,4898 & 0,0418 & 0,3414 & 0,0747 \\
\hline 501 & 122 & 0,2533 & 0,0322 & 0,6321 & 0,0804 & 0,5106 & 0,0322 & 0,3171 & 0,0699 \\
\hline 579 & 123 & 0,2445 & 0,0363 & 0,6278 & 0,0932 & 0,5098 & 0,0363 & 0,3102 & 0,0718 \\
\hline 612 & 124 & 0,2607 & 0,0431 & 0,5932 & 0,098 & 0,5146 & 0,0431 & 0,3231 & 0,0755 \\
\hline 618 & 125 & 0,2739 & 0,0656 & 0,7687 & 0,184 & 0,6028 & 0,0656 & 0,3338 & 0,0902 \\
\hline 473 & 126 & 0,2116 & 0,0474 & 0,4818 & 0,108 & 0,2966 & 0,0474 & 0,2849 & 0,0780 \\
\hline 494 & 127 & 0,3091 & 0,0637 & 0,6613 & 0,1362 & 0,3964 & 0,0637 & 0,3632 & 0,0888 \\
\hline 499 & 128 & 0,2428 & 0,0466 & 0,5871 & 0,1126 & 0,409 & 0,0466 & 0,3088 & 0,0775 \\
\hline 500 & 129 & 0,2386 & 0,0475 & 0,4348 & 0,0865 & 0,3246 & 0,0475 & 0,5593 & 0,1028 \\
\hline 469 & 130 & 0,2147 & 0,0436 & 0,4641 & 0,0943 & 0,4974 & 0,0436 & 0,5496 & 0,1011 \\
\hline pl-61 & 131 & 0,5116 & 0,0689 & 0,8205 & 0,1105 & 0,713 & 0,0689 & 0,7195 & 0,1143 \\
\hline pl-63 & 132 & 0,3907 & 0,0769 & 0,994 & 0,1957 & 0,4634 & 0,0769 & 0,6392 & 0,1193 \\
\hline 197 & 133 & 0,4183 & 0,0578 & 0,8054 & 0,1112 & 0,5631 & 0,0578 & 0,6564 & 0,1079 \\
\hline 210 & 134 & 0,3076 & 0,0547 & 0,9696 & 0,1725 & 0,5126 & 0,0547 & 0,5920 & 0,1063 \\
\hline 309 & 135 & 0,3239 & 0,0455 & 0,9512 & 0,1336 & 0,5618 & 0,0455 & 0,6007 & 0,1019 \\
\hline 341 & 136 & $\mathbf{0 , 2 7 9}$ & 0,0459 & 0,7359 & 0,1211 & 0,6515 & 0,0459 & 0,5777 & 0,1021 \\
\hline 353 & 137 & 0,4599 & 0,0597 & 0,6394 & 0,083 & 0,5572 & 0,0597 & 0,5436 & 0,0806 \\
\hline 282 & 138 & 0,2792 & 0,0442 & 0,6324 & 0,1 & 0,3184 & 0,0442 & 0,4025 & 0,0699 \\
\hline 428 & 139 & 0,2326 & 0,0331 & 0,7245 & 0,1032 & 0,5122 & 0,0331 & 0,3716 & 0,0635 \\
\hline 457 & 140 & 0,226 & 0,0382 & 0,8754 & 0,1481 & 0,4564 & 0,0382 & 0,3675 & 0,0663 \\
\hline 458 & 141 & 0,3033 & 0,0435 & 0,8102 & 0,1161 & 0,5359 & 0,0435 & 0,4195 & 0,0695 \\
\hline 462 & 142 & 0,2167 & 0,0286 & 0,6404 & 0,0845 & 0,2249 & 0,0286 & 0,3619 & 0,0613 \\
\hline
\end{tabular}


Tabela 8: Estimativas dos desvios padrões (d.p.) das estimativas dos parâmetros do modelo de regressão calculadas através da matriz de variâncias e covariâncias robusta, para o ensaio com os cupins.

\begin{tabular}{|c|c|c|c|c|c|c|c|c|c|}
\hline \multirow[t]{2}{*}{ Isolado } & \multirow[t]{2}{*}{$i$} & \multicolumn{2}{|c|}{ multinomial } & \multicolumn{2}{|c|}{ Dirichlet-multinomial } & \multicolumn{2}{|c|}{ REMI } & \multicolumn{2}{|c|}{ REMII } \\
\hline & & e.p. $\left(\hat{\beta}_{1}\right)$ & e.p. $\left(\hat{\beta}_{2}\right)$ & e.p. $\left(\hat{\beta}_{1}\right)$ & e.p. $\left(\hat{\beta}_{2}\right)$ & e.p. $\left(\hat{\beta}_{1}\right)$ & e.p. $\left(\hat{\beta}_{2}\right)$ & e.p. $\left(\hat{\beta}_{1}\right)$ & e.p. $\left(\hat{\beta}_{2}\right)$ \\
\hline 848 & 1 & 0,1709 & 0,0405 & 0,1709 & 0,0405 & 0,1709 & 0,0405 & 0,1709 & 0,0405 \\
\hline 787 & 2 & 0,2183 & 0,0704 & 0,2183 & 0,0704 & 0,2183 & 0,0704 & 0,2183 & 0,0704 \\
\hline 1003 & 3 & 1,0435 & 0,1654 & 1,0435 & 0,1654 & 1,0435 & 0,1654 & 1,0435 & 0,1654 \\
\hline 1006 & 4 & 0,2653 & 0,0712 & 0,2653 & 0,0712 & 0,2653 & 0,0712 & 0,2653 & 0,0712 \\
\hline 1024 & 5 & 0,4035 & 0,0278 & 0,4035 & 0,0278 & 0,4035 & 0,0278 & 0,4035 & 0,0278 \\
\hline 852 & 6 & 0,2247 & 0,0551 & 0,2247 & 0,0551 & 0,2247 & 0,0551 & 0,2247 & 0,0551 \\
\hline 879 & 7 & 0,4194 & 0,0658 & 0,4194 & 0,0658 & 0,4194 & 0,0658 & 0,4194 & 0,0658 \\
\hline 883 & 8 & 0,3931 & 0,0608 & 0,3931 & 0,0608 & 0,3931 & 0,0608 & 0,3931 & 0,0608 \\
\hline 885 & 9 & 0,1575 & 0,0775 & 0,1575 & 0,0775 & 0,1575 & 0,0775 & 0,1575 & 0,0775 \\
\hline 957 & 10 & 0,2889 & 0,0842 & 0,2889 & 0,0842 & 0,2889 & 0,0842 & 0,2889 & 0,0842 \\
\hline 1028 & 11 & 0,6278 & 0,2000 & 0,6278 & 0,2000 & 0,6278 & 0,2000 & 0,6278 & 0,2000 \\
\hline 732 & 12 & 0,3511 & 0,1083 & 0,3511 & 0,1083 & 0,3511 & 0,1083 & 0,3511 & 0,1083 \\
\hline 743 & 13 & 0,8132 & 0,2189 & 0,8132 & 0,2189 & 0,8132 & 0,2189 & 0,8132 & 0,2189 \\
\hline 745 & 14 & 0,6483 & 0,0459 & 0,6483 & 0,0459 & 0,6483 & 0,0459 & 0,6483 & 0,0459 \\
\hline 767 & 15 & 0,2263 & 0,0612 & 0,2263 & 0,0612 & 0,2263 & 0,0612 & 0,2263 & 0,0612 \\
\hline 823 & 16 & 0,2853 & 0,0660 & 0,2853 & 0,0660 & 0,2853 & 0,0660 & 0,2853 & 0,0660 \\
\hline 855 & 17 & 0,3690 & 0,0643 & 0,3690 & 0,0643 & 0,3690 & 0,0643 & 0,3690 & 0,0643 \\
\hline 857 & 18 & 0,5554 & 0,0916 & 0,5554 & 0,0916 & 0,5554 & 0,0916 & 0,5554 & 0,0916 \\
\hline 876 & 19 & 0,5097 & 0,0596 & 0,5097 & 0,0596 & 0,5097 & 0,0596 & 0,5097 & 0,0596 \\
\hline 878 & 20 & 0,1455 & 0,0369 & 0,1455 & 0,0369 & 0,1455 & 0,0369 & 0,1455 & 0,0369 \\
\hline 602 & 21 & 0,4426 & 0,0699 & 0,4426 & 0,0699 & 0,4426 & 0,0699 & 0,4426 & 0,0699 \\
\hline 616 & 22 & 0,8123 & 0,0554 & 0,8123 & 0,0554 & 0,8123 & 0,0554 & 0,8123 & 0,0554 \\
\hline 741 & 23 & 1,0179 & 0,0981 & 1,0179 & 0,0981 & 1,0179 & 0,0981 & 1,0179 & 0,0981 \\
\hline 784 & 24 & 0,7697 & 0,0773 & 0,7697 & 0,0773 & 0,7697 & 0,0773 & 0,7697 & 0,0773 \\
\hline 822 & 25 & 0,3854 & 0,0597 & 0,3854 & 0,0597 & 0,3854 & 0,0597 & 0,3854 & 0,0597 \\
\hline 841 & 26 & 0,3751 & 0,0961 & 0,3751 & 0,0961 & 0,3751 & 0,0961 & 0,3751 & 0,0961 \\
\hline 843 & 27 & 0,3394 & 0,0421 & 0,3394 & 0,0421 & 0,3394 & 0,0421 & 0,3394 & 0,0421 \\
\hline 845 & 28 & 0,3044 & 0,0555 & 0,3044 & 0,0555 & 0,3044 & 0,0555 & 0,3044 & 0,0555 \\
\hline 738 & 29 & 0,5671 & 0,1577 & 0,5671 & 0,1577 & 0,5671 & 0,1577 & 0,5671 & 0,1577 \\
\hline 742 & 30 & 0,2219 & 0,1036 & 0,2219 & 0,1036 & 0,2219 & 0,1036 & 0,2219 & 0,1036 \\
\hline 744 & 31 & 0,5805 & 0,1637 & 0,5805 & 0,1637 & 0,5805 & 0,1637 & 0,5805 & 0,1637 \\
\hline 747 & 32 & 0,4753 & 0,1457 & 0,4753 & 0,1457 & 0,4753 & 0,1457 & 0,4753 & 0,1457 \\
\hline 748 & 33 & 1,0245 & 0,3322 & 1,0245 & 0,3322 & 1,0245 & 0,3322 & 1,0245 & 0,3322 \\
\hline 750 & 34 & 0,8970 & 0,2155 & 0,8970 & 0,2155 & 0,8970 & 0,2155 & 0,8970 & 0,2155 \\
\hline 752 & 35 & 0,8140 & 0,1997 & 0,8140 & 0,1997 & 0,8140 & 0,1997 & 0,8140 & 0,1997 \\
\hline 755 & 36 & 0,5477 & 0,1441 & 0,5477 & 0,1441 & 0,5477 & 0,1441 & 0,5477 & 0,1441 \\
\hline
\end{tabular}


Continuação da Tabela (8). Estimativas dos desvios padrões (d.p.) das estimativas dos parâmetros do modelo de regressão calculadas através da matriz de variâncias e covariâncias robusta, para o ensaio com os cupins.

\begin{tabular}{|c|c|c|c|c|c|c|c|c|c|}
\hline \multirow[t]{2}{*}{ Isolado } & \multirow[t]{2}{*}{$i$} & \multicolumn{2}{|c|}{ multinomial } & \multicolumn{2}{|c|}{ Dirichlet-multinomial } & \multicolumn{2}{|c|}{ REMI } & \multicolumn{2}{|c|}{ REMII } \\
\hline & & e.p. $\left(\hat{\beta}_{1}\right)$ & e.p. $\left(\hat{\beta}_{2}\right)$ & e.p. $\left(\hat{\beta}_{1}\right)$ & e.p. $\left(\hat{\beta}_{2}\right)$ & e.p. $\left(\hat{\beta}_{1}\right)$ & e.p. $\left(\hat{\beta}_{2}\right)$ & e.p. $\left(\hat{\beta}_{1}\right)$ & e.p. $\left(\hat{\beta}_{2}\right)$ \\
\hline 756 & 37 & 1,2687 & 0,3516 & 1,2687 & 0,3516 & 1,2687 & 0,3516 & 1,2687 & 0,3516 \\
\hline 764 & 38 & 0,6239 & 0,1757 & 0,6239 & 0,1757 & 0,6239 & 0,1757 & 0,6239 & 0,1757 \\
\hline 573 & 39 & 0,5099 & 0,0789 & 0,5099 & 0,0789 & 0,5099 & 0,0789 & 0,5099 & 0,0789 \\
\hline 591 & 40 & 0,3589 & 0,0538 & 0,3589 & 0,0538 & 0,3589 & 0,0538 & 0,3589 & 0,0538 \\
\hline 598 & 41 & 0,3682 & 0,0709 & 0,3682 & 0,0709 & 0,3682 & 0,0709 & 0,3682 & 0,0709 \\
\hline 600 & 42 & 0,7475 & 0,1292 & 0,7475 & 0,1292 & 0,7475 & 0,1292 & 0,7475 & 0,1292 \\
\hline 604 & 43 & 0,5623 & 0,1100 & 0,5623 & 0,1100 & 0,5623 & 0,1100 & 0,5623 & 0,1100 \\
\hline 666 & 44 & 0,5952 & 0,0565 & 0,5952 & 0,0565 & 0,5952 & 0,0565 & 0,5952 & 0,0565 \\
\hline 587 & 45 & 0,9550 & 0,2820 & 0,9550 & 0,2820 & 0,9550 & 0,2820 & 0,9550 & 0,2820 \\
\hline 619 & 46 & 0,3495 & 0,0325 & 0,3495 & 0,0325 & 0,3495 & 0,0325 & 0,3495 & 0,0325 \\
\hline 781 & 47 & 0,3557 & 0,0727 & 0,3557 & 0,0727 & 0,3557 & 0,0727 & 0,3557 & 0,0727 \\
\hline 783 & 48 & 0,3425 & 0,0816 & 0,3425 & 0,0816 & 0,3425 & 0,0816 & 0,3425 & 0,0816 \\
\hline 786 & 49 & 0,4439 & 0,0418 & 0,4439 & 0,0418 & 0,4439 & 0,0418 & 0,4439 & 0,0418 \\
\hline 665 & 50 & 0,3879 & 0,1030 & 0,3879 & 0,1030 & 0,3879 & 0,1030 & 0,3879 & 0,1030 \\
\hline 705 & 51 & 0,1526 & 0,0398 & 0,1526 & 0,0398 & 0,1526 & 0,0398 & 0,1526 & 0,0398 \\
\hline 753 & 52 & 1,0594 & 0,2765 & 1,0594 & 0,2765 & 1,0594 & 0,2765 & 1,0594 & 0,2765 \\
\hline 778 & 53 & 0,7848 & 0,2375 & 0,7848 & 0,2375 & 0,7848 & 0,2375 & 0,7848 & 0,2375 \\
\hline 607 & 54 & 0,8351 & 0,0951 & 0,8351 & 0,0951 & 0,8351 & 0,0951 & 0,8351 & 0,0951 \\
\hline 609 & 55 & 0,5126 & 0,1883 & 0,5126 & 0,1883 & 0,5126 & 0,1883 & 0,5126 & 0,1883 \\
\hline 615 & 56 & 0,8667 & 0,2143 & 0,8667 & 0,2143 & 0,8667 & 0,2143 & 0,8667 & 0,2143 \\
\hline 617 & 57 & 0,4314 & 0,0987 & 0,4314 & 0,0987 & 0,4314 & 0,0987 & 0,4314 & 0,0987 \\
\hline 634 & 58 & 0,4932 & 0,0861 & 0,4932 & 0,0861 & 0,4932 & 0,0861 & 0,4932 & 0,0861 \\
\hline 635 & 59 & 0,5199 & 0,1327 & 0,5199 & 0,1327 & 0,5199 & 0,1327 & 0,5199 & 0,1327 \\
\hline 643 & 60 & 0,5538 & 0,1636 & 0,5538 & 0,1636 & 0,5538 & 0,1636 & 0,5538 & 0,1636 \\
\hline 651 & 61 & 0,5816 & 0,1451 & 0,5816 & 0,1451 & 0,5816 & 0,1451 & 0,5816 & 0,1451 \\
\hline 268 & 62 & 0,3265 & 0,1166 & 0,3265 & 0,1166 & 0,3265 & 0,1166 & 0,3265 & 0,1166 \\
\hline 565 & 63 & 0,2582 & 0,0713 & 0,2582 & 0,0713 & 0,2582 & 0,0713 & 0,2582 & 0,0713 \\
\hline 571 & 64 & 0,0831 & 0,0389 & 0,0831 & 0,0389 & 0,0831 & 0,0389 & 0,0831 & 0,0389 \\
\hline 509 & 65 & 0,4719 & 0,0720 & 0,4719 & 0,0720 & 0,4719 & 0,0720 & 0,4719 & 0,0720 \\
\hline 515 & 66 & 0,4663 & 0,0712 & 0,4663 & 0,0712 & 0,4663 & 0,0712 & 0,4663 & 0,0712 \\
\hline 520 & 67 & 0,5949 & 0,0689 & 0,5949 & 0,0689 & 0,5949 & 0,0689 & 0,5949 & 0,0689 \\
\hline 531 & 68 & 0,5270 & 0,0986 & 0,5270 & 0,0986 & 0,5270 & 0,0986 & 0,5270 & 0,0986 \\
\hline 535 & 69 & 0,5500 & 0,0459 & 0,5500 & 0,0459 & 0,5500 & 0,0459 & 0,5500 & 0,0459 \\
\hline 545 & 70 & 1,1391 & 0,1692 & 1,1391 & 0,1692 & 1,1391 & 0,1692 & 1,1391 & 0,1692 \\
\hline 553 & 71 & 0,3864 & 0,0728 & 0,3864 & 0,0728 & 0,3864 & 0,0728 & 0,3864 & 0,0728 \\
\hline 556 & 72 & 0,5588 & 0,1050 & 0,5588 & 0,1050 & 0,5588 & 0,1050 & 0,5588 & 0,1050 \\
\hline 560 & 73 & 0,6589 & 0,1062 & 0,6589 & 0,1062 & 0,6589 & 0,1062 & 0,6589 & 0,1062 \\
\hline
\end{tabular}


Continuação da Tabela (8). Estimativas dos desvios padrões (d.p.) das estimativas dos parâmetros do modelo de regressão calculadas através da matriz de variâncias e covariâncias robusta, para o ensaio com os cupins.

\begin{tabular}{|c|c|c|c|c|c|c|c|c|c|}
\hline \multirow[t]{2}{*}{ Isolado } & \multirow[t]{2}{*}{$i$} & \multicolumn{2}{|c|}{ multinomial } & \multicolumn{2}{|c|}{ Dirichlet-multinomial } & \multicolumn{2}{|c|}{ REMI } & \multicolumn{2}{|c|}{ REMII } \\
\hline & & e.p. $\left(\hat{\beta}_{1}\right)$ & e.p. $\left(\hat{\beta}_{2}\right)$ & e.p. $\left(\hat{\beta}_{1}\right)$ & e.p. $\left(\hat{\beta}_{2}\right)$ & e.p. $\left(\hat{\beta}_{1}\right)$ & e.p. $\left(\hat{\beta}_{2}\right)$ & e.p. $\left(\hat{\beta}_{1}\right)$ & e.p. $\left(\hat{\beta}_{2}\right)$ \\
\hline 585 & 74 & 0,4888 & 0,1015 & 0,4888 & 0,1015 & 0,4888 & 0,1015 & 0,4888 & 0,1015 \\
\hline 532 & 75 & 0,4461 & 0,0643 & 0,4461 & 0,0643 & 0,4461 & 0,0643 & 0,4461 & 0,0643 \\
\hline 554 & 76 & 0,4169 & 0,0950 & 0,4169 & 0,0950 & 0,4169 & 0,0950 & 0,4169 & 0,0950 \\
\hline 555 & 77 & 0,3731 & 0,0344 & 0,3731 & 0,0344 & 0,3731 & 0,0344 & 0,3731 & 0,0344 \\
\hline 558 & 78 & 0,5886 & 0,0965 & 0,5886 & 0,0965 & 0,5886 & 0,0965 & 0,5886 & 0,0965 \\
\hline 562 & 79 & 0,3508 & 0,0991 & 0,3508 & 0,0991 & 0,3508 & 0,0991 & 0,3508 & 0,0991 \\
\hline 566 & 80 & 0,6645 & 0,0963 & 0,6645 & 0,0963 & 0,6645 & 0,0963 & 0,6645 & 0,0963 \\
\hline 569 & 81 & 0,1457 & 0,0494 & 0,1457 & 0,0494 & 0,1457 & 0,0494 & 0,1457 & 0,0494 \\
\hline 572 & 82 & 0,4280 & 0,0595 & 0,4280 & 0,0595 & 0,4280 & 0,0595 & 0,4280 & 0,0595 \\
\hline 574 & 83 & 0,6857 & 0,0905 & 0,6857 & 0,0905 & 0,6857 & 0,0905 & 0,6857 & 0,0905 \\
\hline 448 & 84 & 0,7334 & 0,2460 & 0,7334 & 0,2460 & 0,7334 & 0,2460 & 0,7334 & 0,2460 \\
\hline 534 & 85 & 0,5587 & 0,1826 & 0,5587 & 0,1826 & 0,5587 & 0,1826 & 0,5587 & 0,1826 \\
\hline 536 & 86 & 0,4916 & 0,0711 & 0,4916 & 0,0711 & 0,4916 & 0,0711 & 0,4916 & 0,0711 \\
\hline 538 & 87 & 0,3140 & 0,0843 & 0,3140 & 0,0843 & 0,3140 & 0,0843 & 0,3140 & 0,0843 \\
\hline 542 & 88 & 0,6482 & 0,1355 & 0,6482 & 0,1355 & 0,6482 & 0,1355 & 0,6482 & 0,1355 \\
\hline 544 & 89 & 0,5429 & 0,0320 & 0,5429 & 0,0320 & 0,5429 & 0,0320 & 0,5429 & 0,0320 \\
\hline 548 & 90 & 0,4402 & 0,1541 & 0,4402 & 0,1541 & 0,4402 & 0,1541 & 0,4402 & 0,1541 \\
\hline 549 & 91 & 0,8696 & 0,1292 & 0,8696 & 0,1292 & 0,8696 & 0,1292 & 0,8696 & 0,1292 \\
\hline 550 & 92 & 0,5054 & 0,0840 & 0,5054 & 0,0840 & 0,5054 & 0,0840 & 0,5054 & 0,0840 \\
\hline 391 & 93 & 0,3168 & 0,1222 & 0,3168 & 0,1222 & 0,3168 & 0,1222 & 0,3168 & 0,1222 \\
\hline 484 & 94 & 0,5985 & 0,1172 & 0,5985 & 0,1172 & 0,5985 & 0,1172 & 0,5985 & 0,1172 \\
\hline 485 & 95 & 0,2309 & 0,0384 & 0,2309 & 0,0384 & 0,2309 & 0,0384 & 0,2309 & 0,0384 \\
\hline 489 & 96 & 0,4459 & 0,1138 & 0,4459 & 0,1138 & 0,4459 & 0,1138 & 0,4459 & 0,1138 \\
\hline 498 & 97 & 0,7029 & 0,1356 & 0,7029 & 0,1356 & 0,7029 & 0,1356 & 0,7029 & 0,1356 \\
\hline 512 & 98 & 0,9594 & 0,1595 & 0,9594 & 0,1595 & 0,9594 & 0,1595 & 0,9594 & 0,1595 \\
\hline 524 & 99 & 0,8031 & 0,2667 & 0,8031 & 0,2667 & 0,8031 & 0,2667 & 0,8031 & 0,2667 \\
\hline 529 & 100 & 0,2587 & 0,1066 & 0,2587 & 0,1066 & 0,2587 & 0,1066 & 0,2587 & 0,1066 \\
\hline 476 & 101 & 0,7021 & 0,0875 & 0,7021 & 0,0875 & 0,7021 & 0,0875 & 0,7021 & 0,0875 \\
\hline 672 & 102 & 0,4063 & 0,0883 & 0,4063 & 0,0883 & 0,4063 & 0,0883 & 0,4063 & 0,0883 \\
\hline 760 & 103 & 0,4719 & 0,1984 & 0,4719 & 0,1984 & 0,4719 & 0,1984 & 0,4719 & 0,1984 \\
\hline 969 & 104 & 0,3807 & 0,0776 & 0,3807 & 0,0776 & 0,3807 & 0,0776 & 0,3807 & 0,0776 \\
\hline 986 & 105 & 0,3796 & 0,1003 & 0,3796 & 0,1003 & 0,3796 & 0,1003 & 0,3796 & 0,1003 \\
\hline 987 & 106 & 0,7822 & 0,1294 & 0,7822 & 0,1294 & 0,7822 & 0,1294 & 0,7822 & 0,1294 \\
\hline 988 & 107 & 0,6325 & 0,1193 & 0,6325 & 0,1193 & 0,6325 & 0,1193 & 0,6325 & 0,1193 \\
\hline 1010 & 108 & 0,2157 & 0,0487 & 0,2157 & 0,0487 & 0,2157 & 0,0487 & 0,2157 & 0,0487 \\
\hline 800 & 109 & 0,4886 & 0,0604 & 0,4886 & 0,0604 & 0,4886 & 0,0604 & 0,4886 & 0,0604 \\
\hline
\end{tabular}


Continuação da Tabela (8). Estimativas dos desvios padrões (d.p.) das estimativas dos parâmetros do modelo de regressão calculadas através da matriz de variâncias e covariâncias robusta, para o ensaio com os cupins.

\begin{tabular}{|c|c|c|c|c|c|c|c|c|c|}
\hline \multirow[t]{2}{*}{ Isolado } & \multirow[t]{2}{*}{$i$} & \multicolumn{2}{|c|}{ multinomial } & \multicolumn{2}{|c|}{ Dirichlet-multinomial } & \multicolumn{2}{|c|}{ REMI } & \multicolumn{2}{|c|}{ REMII } \\
\hline & & e.p. $\left(\hat{\beta}_{1}\right)$ & e.p. $\left(\hat{\beta}_{2}\right)$ & e.p. $\left(\hat{\beta}_{1}\right)$ & e.p. $\left(\hat{\beta}_{2}\right)$ & e.p. $\left(\hat{\beta}_{1}\right)$ & e.p. $\left(\hat{\beta}_{2}\right)$ & e.p. $\left(\hat{\beta}_{1}\right)$ & e.p. $\left(\hat{\beta}_{2}\right)$ \\
\hline 868 & 110 & 0,1798 & 0,0870 & 0,1798 & 0,0870 & 0,1798 & 0,0870 & 0,1798 & 0,0870 \\
\hline 888 & 111 & 0,3510 & 0,0728 & 0,3510 & 0,0728 & 0,3510 & 0,0728 & 0,3510 & 0,0728 \\
\hline 900 & 112 & 1,0609 & 0,1880 & 1,0609 & 0,1880 & 1,0609 & 0,1880 & 1,0609 & 0,1880 \\
\hline 940 & 113 & 0,5159 & 0,0664 & 0,5159 & 0,0664 & 0,5159 & 0,0664 & 0,5159 & 0,0664 \\
\hline 620 & 114 & 0,4225 & 0,1646 & 0,4225 & 0,1646 & 0,4225 & 0,1646 & 0,4225 & 0,1646 \\
\hline 641 & 115 & 0,4777 & 0,0786 & 0,4777 & 0,0786 & 0,4777 & 0,0786 & 0,4777 & 0,0786 \\
\hline 649 & 116 & 0,3912 & 0,1126 & 0,3912 & 0,1126 & 0,3912 & 0,1126 & 0,3912 & 0,1126 \\
\hline 663 & 117 & 0,4400 & 0,0879 & 0,4400 & 0,0879 & 0,4400 & 0,0879 & 0,4400 & 0,0879 \\
\hline 669 & 118 & 0,4331 & 0,1012 & 0,4331 & 0,1012 & 0,4331 & 0,1012 & 0,4331 & 0,1012 \\
\hline 694 & 119 & 0,3276 & 0,0918 & 0,3276 & 0,0918 & 0,3276 & 0,0918 & 0,3276 & 0,0918 \\
\hline 704 & 120 & 0,2573 & 0,0542 & 0,2573 & 0,0542 & 0,2573 & 0,0542 & 0,2573 & 0,0542 \\
\hline 292 & 121 & 0,6455 & 0,0969 & 0,6455 & 0,0969 & 0,6455 & 0,0969 & 0,6455 & 0,0969 \\
\hline 501 & 122 & 0,5351 & 0,0777 & 0,5351 & 0,0777 & 0,5351 & 0,0777 & 0,5351 & 0,0777 \\
\hline 579 & 123 & 0,6366 & 0,0993 & 0,6366 & 0,0993 & 0,6366 & 0,0993 & 0,6366 & 0,0993 \\
\hline 612 & 124 & 0,4667 & 0,0614 & 0,4667 & 0,0614 & 0,4667 & 0,0614 & 0,4667 & 0,0614 \\
\hline 618 & 125 & 0,8699 & 0,2423 & 0,8699 & 0,2423 & 0,8699 & 0,2423 & 0,8699 & 0,2423 \\
\hline 473 & 126 & 0,6103 & 0,1759 & 0,6103 & 0,1759 & 0,6103 & 0,1759 & 0,6103 & 0,1759 \\
\hline 494 & 127 & 0,2760 & 0,0667 & 0,2760 & 0,0667 & 0,2760 & 0,0667 & 0,2760 & 0,0667 \\
\hline 499 & 128 & 0,6962 & 0,1490 & 0,6962 & 0,1490 & 0,6962 & 0,1490 & 0,6962 & 0,1490 \\
\hline 500 & 129 & 0,3200 & 0,0320 & 0,3200 & 0,0320 & 0,3200 & 0,0320 & 0,3200 & 0,0320 \\
\hline 469 & 130 & 0,7355 & 0,0984 & 0,7355 & 0,0984 & 0,7355 & 0,0984 & 0,7355 & 0,0984 \\
\hline pl-61 & 131 & 0,4830 & 0,1150 & 0,4830 & 0,1150 & 0,4830 & 0,1150 & 0,4830 & 0,1150 \\
\hline pl-63 & 132 & 0,5975 & 0,1656 & 0,5975 & 0,1656 & 0,5975 & 0,1656 & 0,5975 & 0,1656 \\
\hline 197 & 133 & 0,2983 & 0,0477 & 0,2983 & 0,0477 & 0,2983 & 0,0477 & 0,2983 & 0,0477 \\
\hline 210 & 134 & 0,6473 & 0,1708 & 0,6473 & 0,1708 & 0,6473 & 0,1708 & 0,6473 & 0,1708 \\
\hline 309 & 135 & 0,3936 & 0,0701 & 0,3936 & 0,0701 & 0,3936 & 0,0701 & 0,3936 & 0,0701 \\
\hline 341 & 136 & 0,3132 & 0,0955 & 0,3132 & 0,0955 & 0,3132 & 0,0955 & 0,3132 & 0,0955 \\
\hline 353 & 137 & 0,3038 & 0,0414 & 0,3038 & 0,0414 & 0,3038 & 0,0414 & 0,3038 & 0,0414 \\
\hline 282 & 138 & 0,2885 & 0,0588 & 0,2885 & 0,0588 & 0,2885 & 0,0588 & 0,2885 & 0,0588 \\
\hline 428 & 139 & 0,6261 & 0,0525 & 0,6261 & 0,0525 & 0,6261 & 0,0525 & 0,6261 & 0,0525 \\
\hline 457 & 140 & 0,5745 & 0,1430 & 0,5745 & 0,1430 & 0,5745 & 0,1430 & 0,5745 & 0,1430 \\
\hline 458 & 141 & 0,4410 & 0,0839 & 0,4410 & 0,0839 & 0,4410 & 0,0839 & 0,4410 & 0,0839 \\
\hline 462 & 142 & 0,3980 & 0,0739 & 0,3980 & 0,0739 & 0,3980 & 0,0739 & 0,3980 & 0,0739 \\
\hline
\end{tabular}




\subsection{Resultados para o ensaio com o ácaro T. urticae:}

Os resultados apresentados nesta Secção foram obtidos utilizando-se os dados do ensaio com o ácaro T. urticae, conforme descrito na Seção 3 com o uso do preditor linear descrito em (9).

Observa-se que pelas expressões (17), (19), (24) e (32) os valores esperados para o vetor de resposta multinomial acumulada é o mesmo entre todos os modelos: multinomial, DM, REMI e REMII, não importando assim qual seja a suposição feita para a estrutura da matriz de variâncias e covariâncias, por exemplo (18), (20), (27) ou (35).

Como esperado, as estimativas dos parâmetros do modelo de regressão, $\beta_{1 i}$ e $\beta_{2 i}$, são as mesmas para todos os modelos mas os desvios padrões das estimativas mudam de acordo com os modelos. As Figuras 11 a 16 mostram os valores observados e a curva estimada para os 80 isolados utilizados no ensaio com os ácaros.

Pode-se observar pelas Figuras 11 a 16 que, entre as réplicas, existe uma variabilidade mais acentuada no coeficiente angular da reta do que no intercepto, para alguns isolados. Fato esse ressaltado pelos resultados de $\hat{\nu}_{i}^{2}$, apresentados na Tabela 10. Essas estimativas de $\nu_{i}^{2}$ foram bem próximas de zero. Isto também foi visto na Tabela 9. Este fato foi observado para todos os 80 isolados, mostrando assim que seria razoável utilizar modelos que permitam efeitos aleatórios apenas para o coeficiente angular $\left(\beta_{2 i}\right)$. O que se observa para os resultados do ensaio com o ácaro, diferente do que ocorre com os resultados do ensaio com o cupim, é uma consistência maior no comportamento da mortalidade acumulada, pois existe uma menor variabilidade entre as repetições dentro de cada isolado.

Pelo diagrama de dispersão dos desvios padrões dos $\hat{\beta}^{\prime}$ s do modelo multinomial versus os desvios padrões dos $\hat{\beta}^{\prime}$ s dos modelos DM, REMI e REMII, visto na Figura 17 (a,b), nota-se que ignorar a presença da variação extra-multinomial pode conduzir a uma subestimação nas estimativas dos desvios padrões dos $\hat{\beta}^{\prime}$ s. Esse fato tende a subestimar o erro padrão verdadeiro das estimativas dos efeitos dos isolados. Também observa-se que o modelo de Dirichlet-multinomial parece superestimar 
o d.p. $(\hat{\beta})$ quando comparado com o modelo multinomial e os dois modelos de efeitos aleatórios, REMI e REMII, mas não de uma maneira tão acentuada como aconteceu com os dados do bioensaio dos cupins.

A Figura 17 (b) mostra a igualdade dos resultados da matriz de variâncias e covariâncias robusta entre todos os modelos. Isto não foi um resultado esperado, mas parece ser causado pela estrutura especial do predictor linear (9), que possui apenas o dia como variável regressora. O mesmo padrão de comportamento foi encontrado com os dados apresentados por O'Hara Hines (1993), quando excluídas todas as covariáveis do modelo e ajustando somente a regressão no tempo.

As Figuras $18(a-d)$ mostram como as distribuições das estimativas dos parâmetros de superdispersão apresentam um comportamento assimétrico. Isto também pode ser visto na Tabela 9. Se considerarmos os modelos com superdispersão constante tem-se que a estimativa do parâmetro de superdispersão para $\rho$ é 0.0872 (modelo DM), para $\sigma^{2}, 0.5241$ (modelo REMI), para $\nu^{2}, 0.0006$ e para $\tau^{2}$ é 0.1663 (modelo REMII). Também é possível observar, pela Figura $10(a)$, pelas estatísticas apresentadas na Tabela 9 e pelos resultados apresentados na Tabela 10, que não existe evidência de superdispersão $(\hat{\rho}<0)$ em vinte e um dos isolados analisados, o que corresponde, aproximadamente, à quarta parte do número de isolados analisados. Isso parece indicar que o modelo DM, nesse conjunto de dados, modela a superdispersão de maneira satisfatória.

Na Figura $18(f, g)$ nota-se que, para muitos dos 80 isolados utilizados no ensaio dos ácaros, o tempo médio letal $\left(L T_{50}\right)$ é superior a 6 dias, o último dia avaliado no ensaio. Esse fato é ainda mais agravante quando se observa o tempo letal 90, $L T_{90}$, indicando a necessidade de continuação do experimento durante mais dias para se obter uma estimativa melhor do modelo. Segundo os entomologistas, isto é contraposto ao fato de que após alguns dias, os ácaros tendem a criar uma resistência aos isolados, criando assim uma resistência natural ao patógeno, mostrando que o prolongamento do ensaio por mais dias pode vir a prejudicar o ajuste dos modelos. $\mathrm{O}$ pesquisadorr está interessado em agrupar os isolados em três grupos: alta virulência, 
média virulência e virulência fraca para poder identificar os patógenos mais virulentos que possam ser usados nos experimentos de campo. Assim, um método estatístico adequado para agrupar os isolados seria um que fosse robusto à falta de informação para a estimação de alguns modelos. A análise feita neste trabalho não está completa há necessidade de se continuá-la.

Tabela 9: Sumário das estatísticas descritivas para os parâmetros de superdispersão dos modelos DM, REMI e REMII, nos quais a superdispersão varia entre os isolados, considerando-se o ensaio com os ácaros.

\begin{tabular}{ccccccc}
\hline \multirow{2}{*}{ Modelo } & Parâmetro de & \multicolumn{5}{c}{ Estimativas } \\
\cline { 3 - 7 } & superdispersão & média (d.p.) & Mediana & Min. & Max. & CV \% \\
\hline DM & $\rho_{i}$ & $0,0538(0,1077)$ & 0,0270 & $-0,0480$ & 0,6923 & 200,19 \\
\hline REMI & $\sigma_{i}^{2}$ & $0,3698(0,4193)$ & 0,1901 & 0,0001 & 2,3689 & 113,39 \\
\hline REMII & $\nu_{i}^{2}$ & $3,27 \mathrm{e}-07(1,12 \mathrm{e}-07)$ & $2,73 \mathrm{e}-12$ & $3,21 \mathrm{e}-19$ & $6,57 \mathrm{e}-07$ & 3,43 \\
& $\tau_{i}^{2}$ & $0,0100(0.0219)$ & 0,0001 & $5,80 \mathrm{e}-15$ & 0,1201 & 219,00 \\
\hline
\end{tabular}

A Figure $18(h)$ mostra que a correlação entre o intercepto e o coeficiente angular da reta é negativa e isto é confirmado pelo histograma (e).

Apesar de não dispormos de um teste estatśtico que discrimine entre os modelos considerados neste trabalho, recomendamos, para esse conjunto de dados de ácaros, o uso do modelo com efeitos aleatórios no intercepto e no coeficiente angular da reta (REMI). Pelo fato desse modelo ter apresentado resultados mais consistentes e coerentes. 
Tabela 10: Estimativas dos parâmetros da regressão e dos parâmetros de superdispersão para o ensaio com os ácaros.

\begin{tabular}{|c|c|c|c|c|c|c|c|c|c|c|}
\hline Isolados & $i$ & $\hat{\beta}_{1}$ & $\hat{\beta}_{2}$ & $\hat{\boldsymbol{\rho}}$ & $\hat{\sigma}^{2}$ & $\hat{\nu}^{2}$ & $\hat{\boldsymbol{\tau}}^{2}$ & $\hat{\lambda}$ & $L \hat{L T}_{50}$ & $\hat{L T}_{90}$ \\
\hline 756 & 1 & $-5,3049$ & 0,8264 & $-0,0325$ & 0,0000 & 0,0000 & 0,0000 & $-0,8947$ & 6,4193 & 9,0782 \\
\hline 548 & 2 & $-4,8913$ & 0,8382 & 0,0662 & 0,2333 & 0,0000 & 0,0000 & $-0,8920$ & 5,8352 & 8,4565 \\
\hline 549 & 3 & $-5,7298$ & 0,9948 & $-0,0019$ & 0,1010 & 0,0000 & 0,0044 & $-0,9272$ & 5,7596 & 7,9682 \\
\hline 760 & 4 & $-5,5864$ & 0,9344 & 0,0310 & 0,7045 & 0,0000 & 0,0001 & $-0,9507$ & 5,9784 & 8,3298 \\
\hline 551 & 5 & $-5,1459$ & 1,1762 & 0,2329 & 2,3689 & 0,0000 & 0,1201 & $-0,7572$ & 4,3750 & 6,2431 \\
\hline 900 & 6 & $-5,7651$ & 0,9454 & 0,0248 & 0,0001 & 0,0000 & 0,0000 & $-0,9755$ & 6,0979 & 8,4219 \\
\hline 600 & 7 & $-7,5051$ & 1,8972 & 0,1709 & 1,3563 & 0,0000 & 0,0882 & $-0,9480$ & 3,9558 & 5,1139 \\
\hline 757 & 8 & $-5,2034$ & 0,8460 & 0,1527 & 0,6172 & 0,0000 & 0,0000 & $-0,6724$ & 6,1505 & 8,7476 \\
\hline 1202 & 9 & $-9,6598$ & 1,4961 & $-0,0480$ & 0,0597 & 0,0000 & 0,0016 & $-0,9836$ & 6,4567 & 7,9254 \\
\hline 635 & 10 & $-4,6348$ & 0,8713 & 0,0261 & 0,0000 & 0,0000 & 0,0000 & $-0,9264$ & 5,3197 & 7,8416 \\
\hline 749 & 11 & $-5,2929$ & 0,9781 & 0,0346 & 0,0987 & 0,0000 & 0,0037 & $-0,8963$ & 5,4116 & 7,6582 \\
\hline 1197 & 12 & $-5,4249$ & 0,9626 & 0,0181 & 0,4117 & 0,0000 & 0,0001 & $-0,9367$ & 5,6355 & 7,9180 \\
\hline 196 & 13 & $-4,7852$ & 0,8525 & 0,0482 & 0,0000 & 0,0000 & 0,0000 & $-0,9235$ & 5,6130 & 8,1903 \\
\hline 252 & 14 & $-4,7763$ & 0,9352 & 0,1082 & 0,7355 & 0,0000 & 0,0002 & $-0,9299$ & 5,1074 & 7,4570 \\
\hline 476 & 15 & $-5,3129$ & 0,7382 & 0,0197 & 0,4522 & 0,0000 & 0,0134 & $-0,8724$ & 7,1971 & 10,1735 \\
\hline 494 & 16 & $-9,6860$ & 3,3609 & 0,5846 & 1,4563 & 0,0000 & 0,0003 & $-0,8860$ & 2,8820 & 3,5357 \\
\hline 1006 & 17 & $-5,7133$ & 1,2773 & 0,0682 & 1,0643 & 0,0000 & 0,0524 & $-0,9347$ & 4,4728 & 6,1929 \\
\hline 876 & 18 & $-4,9047$ & 0,7309 & 0,0419 & 0,1355 & 0,0000 & 0,0000 & $-0,8171$ & 6,7106 & 9,7169 \\
\hline 890 & 19 & $-4,6435$ & 0,7731 & 0,0531 & 0,0084 & 0,0000 & 0,0011 & $-0,8085$ & 6,0066 & 8,8488 \\
\hline 893 & 20 & $-5,4338$ & 0,8542 & 0,0185 & 0,1331 & 0,0000 & 0,0000 & $-0,8250$ & 6,3612 & 8,9334 \\
\hline 895 & 21 & $-5,0540$ & 0,8387 & 0,0410 & 0,3413 & 0,0000 & 0,0113 & $-0,7788$ & 6,0260 & 8,6458 \\
\hline 881 & 22 & $-4,6647$ & 0,7336 & 0,0878 & 0,1210 & 0,0000 & 0,0000 & $-0,7073$ & 6,3587 & 9,3539 \\
\hline 887 & 23 & $-6,2519$ & 1,2283 & 0,0162 & 0,1003 & 0,0000 & 0,0054 & $-0,9732$ & 5,0899 & 6,8787 \\
\hline 612 & 24 & $-7,0724$ & 1,5297 & 0,0102 & 0,0084 & 0,0000 & 0,0000 & $-0,9855$ & 4,6234 & 6,0598 \\
\hline 931 & 25 & $-6,1360$ & 1,2025 & $-0,0006$ & 0,0853 & 0,0000 & 0,0046 & $-0,9353$ & 5,1029 & 6,9302 \\
\hline 764 & 26 & $-5,4567$ & 1,0908 & 0,0133 & 0,1374 & 0,0000 & 0,0000 & $-0,9288$ & 5,0022 & 7,0164 \\
\hline 908 & 27 & $-10,0235$ & 3,1388 & 0,0166 & 0,2764 & 0,0000 & 0,0309 & $-0,7427$ & 3,1934 & 3,8934 \\
\hline 986 & 28 & $-5,7567$ & 1,1909 & $-0,0115$ & 0,0000 & 0,0000 & 0,0000 & $-0,9259$ & 4,8341 & 6,6792 \\
\hline 969 & 29 & $-11,2185$ & 4,0312 & 0,6923 & 0,7227 & 0,0000 & 0,0764 & $-0,9257$ & 2,7829 & 3,3280 \\
\hline 987 & 30 & $-6,4859$ & 1,3894 & 0,0092 & 0,5209 & 0,0000 & 0,0000 & $-0,8249$ & 4,6682 & 6,2496 \\
\hline 902 & 31 & $-4,3675$ & 1,0158 & 0,1071 & 0,7342 & 0,0000 & 0,0384 & $-0,8219$ & 4,2996 & 6,4626 \\
\hline 498 & 32 & $-6,6380$ & 1,4120 & 0,0430 & 0,2742 & 0,0000 & 0,0000 & $-0,9646$ & 4,7010 & 6,2570 \\
\hline 499 & 33 & $-6,2095$ & 1,2657 & $-0,0027$ & 0,0846 & 0,0000 & 0,0032 & $-0,9262$ & 4,9060 & 6,6420 \\
\hline 500 & 34 & $-5,4400$ & 1,1577 & 0,0606 & 0,7567 & 0,0000 & 0,0001 & $-0,6273$ & 4,6990 & 6,5969 \\
\hline 462 & 35 & $-4,4079$ & 1,2180 & 0,1048 & 0,6039 & 0,0000 & 0,0364 & $-0,8834$ & 3,6188 & 5,4227 \\
\hline 522 & 36 & $-5,4149$ & 0,9806 & $-0,0036$ & 0,0000 & 0,0000 & 0,0000 & $-0,9433$ & 5,5223 & 7,7631 \\
\hline 589 & 37 & $-4,6424$ & 0,8957 & 0,0267 & 0,0370 & 0,0000 & 0,0018 & $-0,6538$ & 5,1828 & 7,6358 \\
\hline 520 & 38 & $-5,5490$ & 1,0085 & $-0,0187$ & 0,0089 & 0,0000 & 0,0000 & $-0,8604$ & 5,5022 & 7,6809 \\
\hline 529 & 39 & $-5,2451$ & 0,8551 & 0,0373 & 0,1671 & 0,0000 & 0,0051 & $-0,9439$ & 6,1336 & 8,7030 \\
\hline 643 & 40 & $-5,6992$ & 1,3257 & 0,1302 & 1,0492 & 0,0000 & 0,0007 & $-0,9508$ & 4,2989 & 5,9562 \\
\hline
\end{tabular}


Continuação da Tabela (10). Estimativas dos parâmetros da regressão e dos parâmetros de superdispersão para o ensaio com os ácaros.

\begin{tabular}{|c|c|c|c|c|c|c|c|c|c|c|}
\hline Isolados & $i$ & $\hat{\beta}_{1}$ & $\hat{\beta}_{2}$ & $\hat{\boldsymbol{\rho}}$ & $\hat{\sigma}^{2}$ & $\hat{\nu}^{2}$ & $\hat{\tau}^{2}$ & $\hat{\lambda}$ & $\hat{L T}_{50}$ & $L \hat{T} T_{90}$ \\
\hline 868 & 41 & $-4,6086$ & 2,2384 & 0,1430 & 0,2095 & 0,0000 & 0,0497 & $-0,8725$ & 2,0589 & 3,0405 \\
\hline 1058 & 42 & $-4,7587$ & 1,0232 & 0,0824 & 0,4666 & 0,0000 & 0,0001 & $-0,9310$ & 4,6507 & 6,7981 \\
\hline 1078 & 43 & $-4,7127$ & 1,1385 & 0,0424 & 1,0101 & 0,0000 & 0,0537 & $-0,4566$ & 4,1395 & 6,0695 \\
\hline pl61 & 44 & $-7,6864$ & 1,1608 & $-0,0088$ & 0,9693 & 0,0000 & 0,0001 & $-0,9599$ & 6,6217 & 8,5146 \\
\hline 641 & 45 & $-5,1500$ & 0,8439 & 0,0217 & 0,1901 & 0,0000 & 0,0067 & $-0,7922$ & 6,1025 & 8,7061 \\
\hline 642 & 46 & $-5,0866$ & 0,9739 & 0,0729 & 0,1377 & 0,0000 & 0,0000 & $-0,8129$ & 5,2230 & 7,4791 \\
\hline 722 & 47 & $-5,3912$ & 1,0368 & 0,0958 & 1,1063 & 0,0000 & 0,0459 & $-0,9596$ & 5,1999 & 7,3192 \\
\hline 307 & 48 & $-3,6120$ & 1,3446 & 0,1208 & 0,0151 & 0,0000 & 0,0000 & $-0,8361$ & 2,6864 & 4,3205 \\
\hline 457 & 49 & $-6,2698$ & 1,9342 & 0,0007 & 0,2278 & 0,0000 & 0,0192 & $-0,9422$ & 3,2415 & 4,3775 \\
\hline 485 & 50 & $-5,8517$ & 0,7996 & $-0,0043$ & 0,6692 & 0,0000 & 0,0000 & $-0,9212$ & 7,3183 & 10,0663 \\
\hline 489 & 51 & $-4,9075$ & 1,4642 & 0,0651 & 0,5802 & 0,0000 & 0,0417 & $-0,9343$ & 3,3518 & 4,8525 \\
\hline 618 & 52 & $-4,9311$ & 1,2580 & 0,1078 & 0,0522 & 0,0000 & 0,0000 & $-0,9432$ & 3,9198 & 5,6664 \\
\hline 620 & 53 & $-4,1783$ & 0,6788 & 0,0418 & 0,1467 & 0,0000 & 0,0062 & $-0,7508$ & 6,1559 & 9,3930 \\
\hline 787 & 54 & $-6,1976$ & 1,2919 & 0,0270 & 0,4466 & 0,0000 & 0,0001 & $-0,9655$ & 4,7974 & 6,4982 \\
\hline 268 & 55 & $-6,2002$ & 0,9669 & $-0,0140$ & 0,0983 & 0,0000 & 0,0028 & $-0,9188$ & 6,4121 & 8,6845 \\
\hline 309 & 56 & $-6,0947$ & 1,0015 & $-0,0111$ & 0,0496 & 0,0000 & 0,0000 & $-0,8172$ & 6,0855 & 8,2794 \\
\hline 498 & 57 & $-4,9514$ & 0,9889 & 0,0465 & 0,6691 & 0,0000 & 0,0307 & $-0,8656$ & 5,0070 & 7,2289 \\
\hline 501 & 58 & $-5,7744$ & 0,8964 & 0,0061 & 0,4032 & 0,0000 & 0,0000 & $-0,9501$ & 6,4421 & 8,8934 \\
\hline 695 & 59 & $-6,6116$ & 1,1407 & $-0,0140$ & 0,1868 & 0,0000 & 0,0059 & $-0,9432$ & 5,7959 & 7,7221 \\
\hline pl63 & 60 & $-5,6887$ & 1,8542 & 0,0599 & 0,5092 & 0,0000 & 0,0000 & $-0,7956$ & 3,0680 & 4,2530 \\
\hline 759 & 61 & $-4,5822$ & 0,7971 & 0,0334 & 0,4410 & 0,0000 & 0,0148 & $-0,7450$ & 5,7487 & 8,5052 \\
\hline 532 & 62 & $-4,1336$ & 0,7061 & 0,0490 & 0,1779 & 0,0000 & 0,0000 & $-0,8002$ & 5,8543 & 8,9662 \\
\hline 210 & 63 & $-4,9623$ & 0,9148 & 0,0202 & 0,0852 & 0,0000 & 0,0048 & $-0,9204$ & 5,4242 & 7,8260 \\
\hline 468 & 64 & $-5,0674$ & 1,2262 & 0,0799 & 0,4644 & 0,0000 & 0,0001 & $-0,9546$ & 4,1326 & 5,9245 \\
\hline 759 & 65 & $-5,0066$ & 0,9980 & 0,0091 & 0,0000 & 0,0000 & 0,0000 & $-0,8106$ & 5,0165 & 7,2180 \\
\hline 561 & 66 & $-4,7143$ & 0,7981 & 0,0270 & 0,0517 & 0,0000 & 0,0000 & $-0,9480$ & 5,9069 & 8,6600 \\
\hline 755 & 67 & $-4,7632$ & 0,7036 & 0,0529 & 0,1413 & 0,0000 & 0,0049 & $-0,7705$ & 6,7694 & 9,8921 \\
\hline 1192 & 68 & $-4,2344$ & 0,7109 & 0,1836 & 1,0982 & 0,0000 & 0,0000 & $-0,7843$ & 5,9567 & 9,0476 \\
\hline 569 & 69 & $-5,9458$ & 0,9498 & $-0,0085$ & 0,0461 & 0,0000 & 0,0020 & $-0,9510$ & 6,2601 & 8,5735 \\
\hline 573 & 70 & $-5,9274$ & 1,0573 & $-0,0169$ & 0,0000 & 0,0000 & 0,0000 & $-0,9857$ & 5,6065 & 7,6847 \\
\hline 579 & 71 & $-5,5432$ & 1,0147 & $-0,0067$ & 0,0000 & 0,0000 & 0,0000 & $-0,9733$ & 5,4631 & 7,6285 \\
\hline 590 & 72 & $-5,3737$ & 0,8049 & 0,0785 & 0,5758 & 0,0000 & 0,0000 & $-0,7568$ & 6,6760 & 9,4057 \\
\hline 615 & 73 & $-4,7838$ & 0,7554 & $-0,0030$ & 0,0674 & & 0,0026 & $-0,9329$ & 6,3326 & 9,2412 \\
\hline 1103 & 74 & $-5,4401$ & 0,5999 & 0,0381 & 0,1546 & 0,0000 & 0,0000 & $-0,9899$ & 7,6011 & 9,9847 \\
\hline 619 & 75 & $-7,0068$ & 0,9218 & $-0,0287$ & 0,3427 & 0,0000 & 0,0052 & $-0,7944$ & 6,2262 & 8,4503 \\
\hline 1010 & 76 & $-6,1509$ & 0,9879 & 0,0043 & 0,6396 & 0,0000 & 0,0000 & $-0,8907$ & 5,4674 & 7,4756 \\
\hline 1108 & 77 & $-5,9819$ & 1,0941 & 0,0165 & 0,2914 & 0,0000 & 0,0000 & $-0,9779$ & 7,4308 & 9,9530 \\
\hline 644 & 78 & $-6,4734$ & 0,8712 & $-0,0206$ & 0,4156 & 0,0000 & 0,0036 & $-0,9737$ & 6,9654 & 9,5945 \\
\hline 647 & 79 & $-5,8213$ & 0,8357 & $-0,0060$ & 0,1588 & 0,0000 & 0,0000 & $-0,9750$ & 7,7284 & 10,6553 \\
\hline 739 & 80 & $-5,8016$ & 0,7507 & $-0,0277$ & 0,0000 & 0,0000 & 0,0000 & $-0,9775$ & 9,0689 & 12,7318 \\
\hline
\end{tabular}


Isolado 756

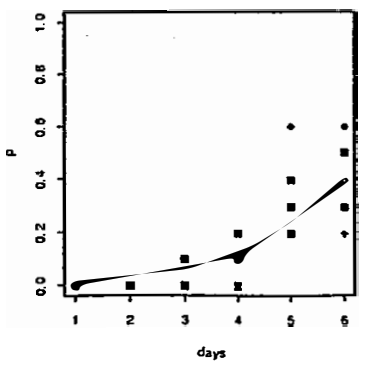

Isolado 760

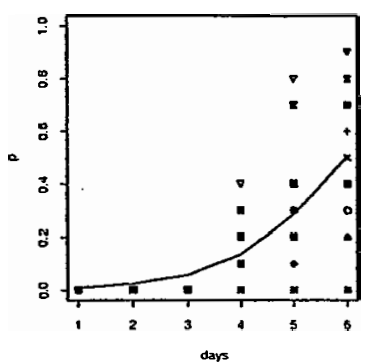

Isolado 600

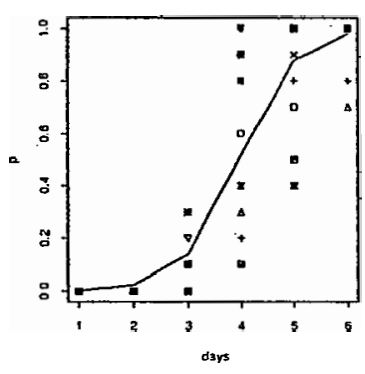

Isolado 635

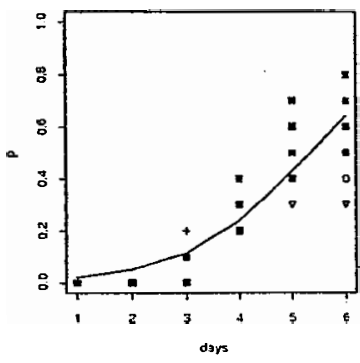

Isolado 196

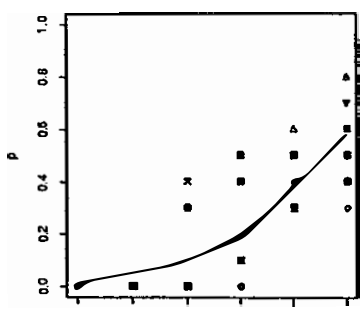

Isolado 548

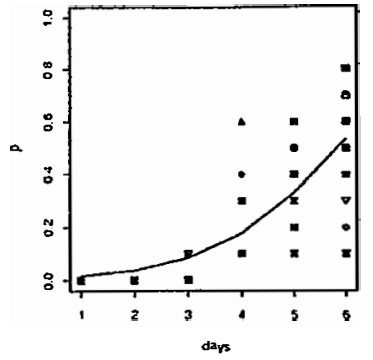

Isolado 551

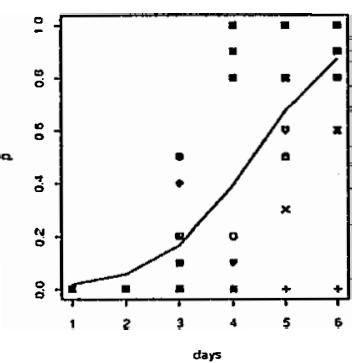

Isolado 757

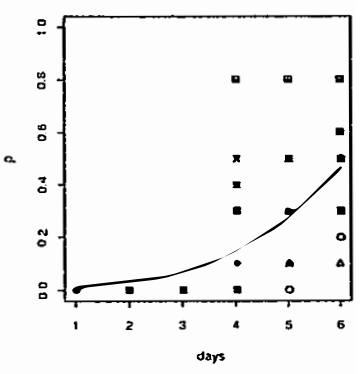

Isolado 749

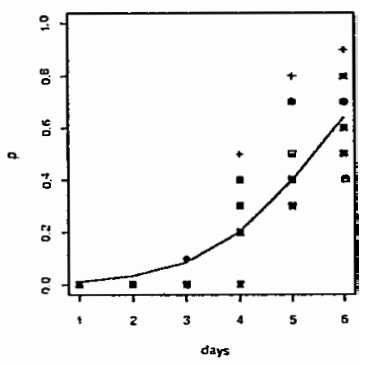

Isolado 252

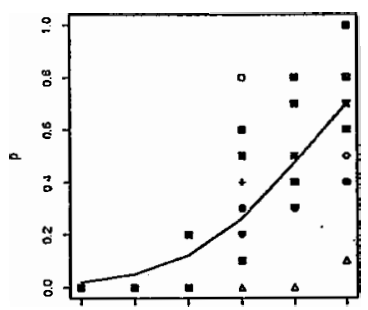

Isolado 549

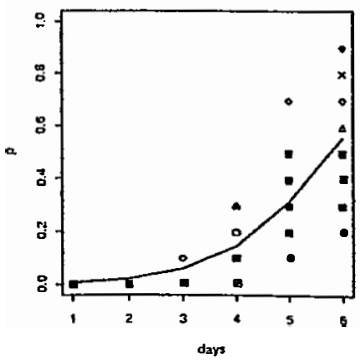

Isolado 900

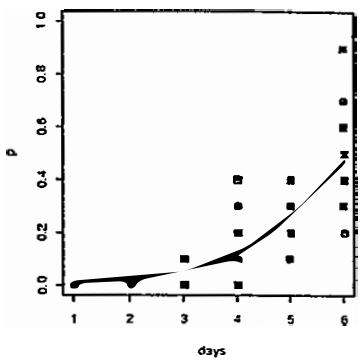

Isolado 1202

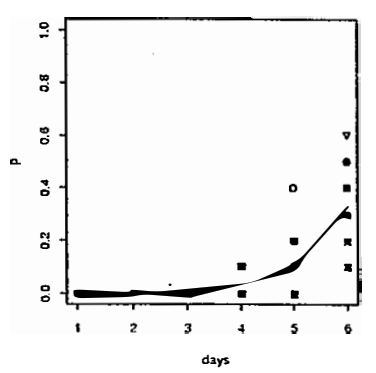

Isolado 1197

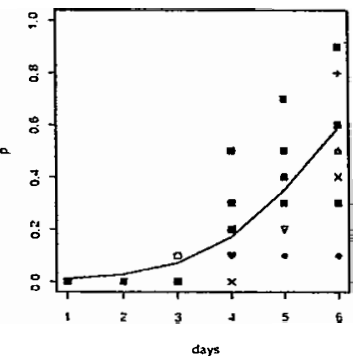

Isolado 476

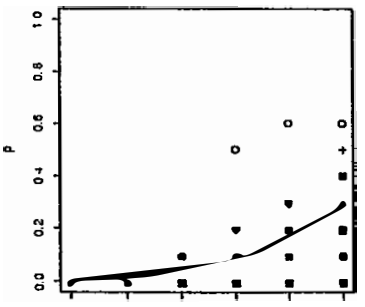

Figura 11: Proporção da mortalidade acumulada durante os 6 dias, para quinze isolados do experimento com o ácaro T. urticae. 


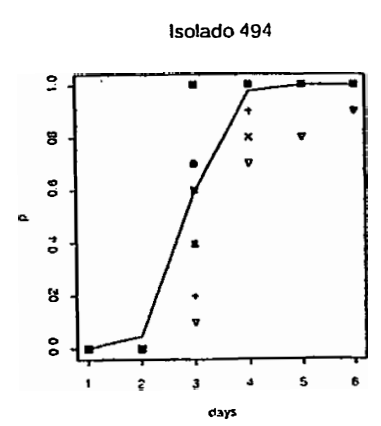

Isolado 890

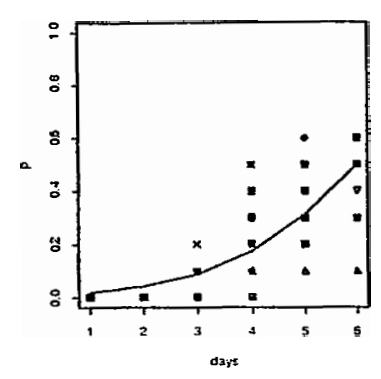

Iso'ado 881

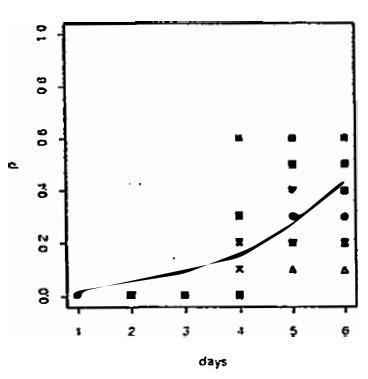

Isolado 931

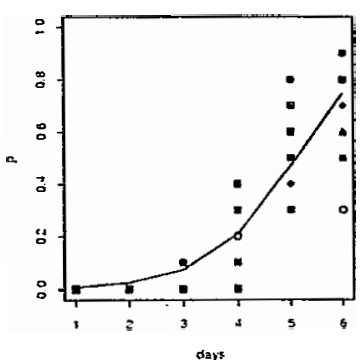

Isolado 986

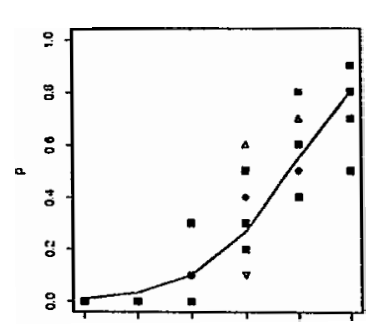

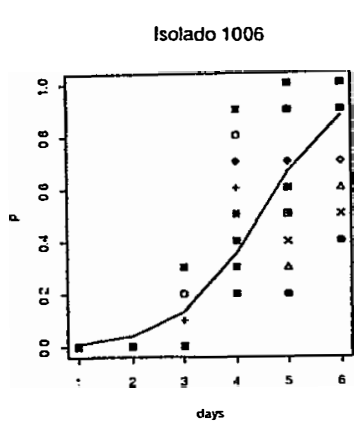

Isolado 893

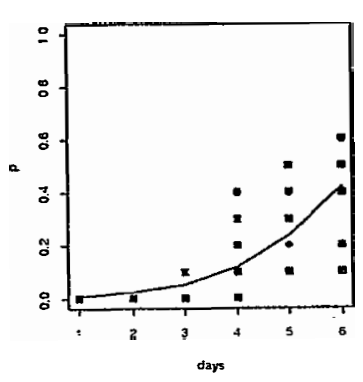

Isolado 887

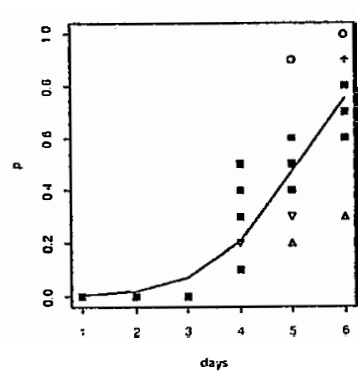

Isolado 764

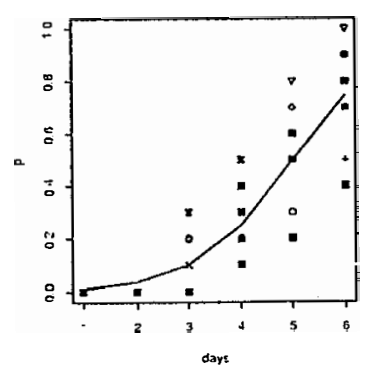

Isolado 969

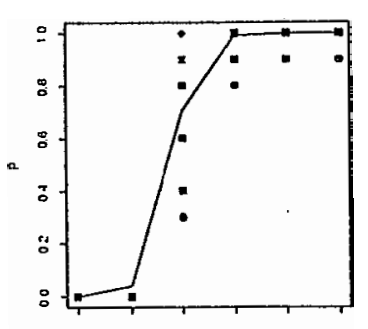

Isolado 876

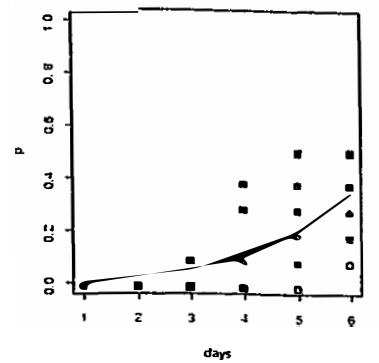

Isolado 895

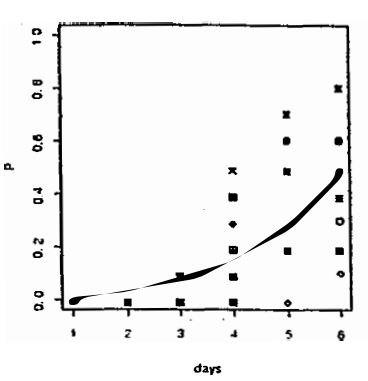

isolado 612

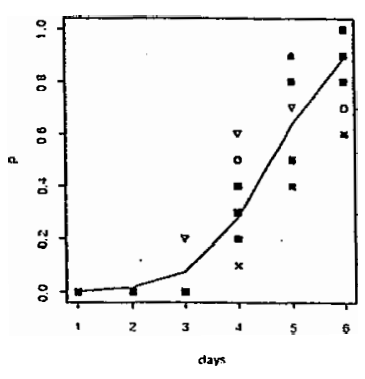

Isolado 908

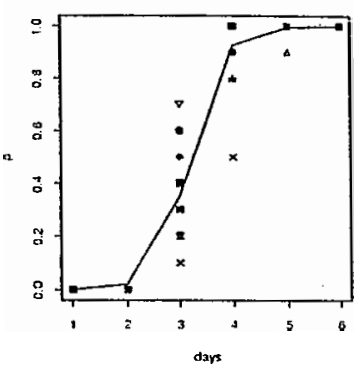

Isolado 987

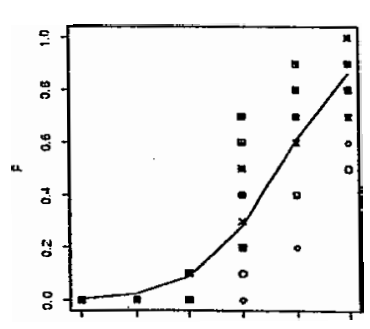

Figura 12: Proporção da mortalidade acumulada durante os 6 dias, para quinze isolados do experimento com o ácaro T. urticae. 
Isolado 902

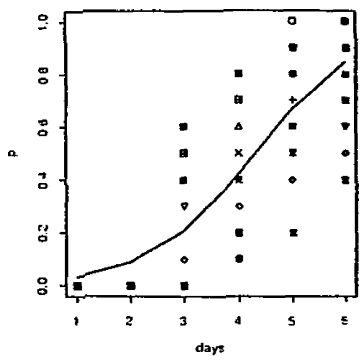

Isolado 500

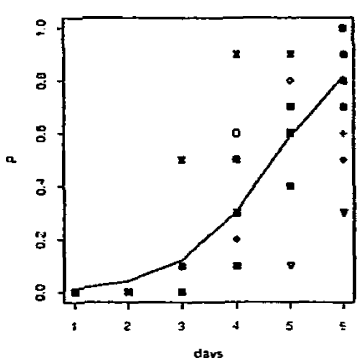

Isolado 589

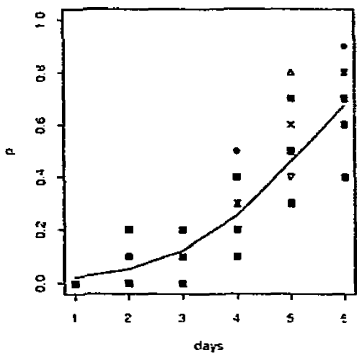

Isolado 643

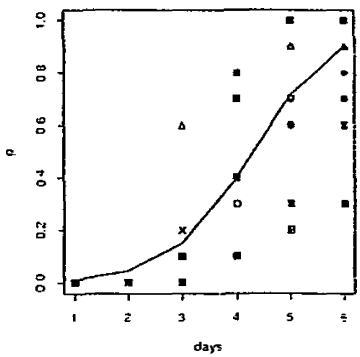

Isolado 1078

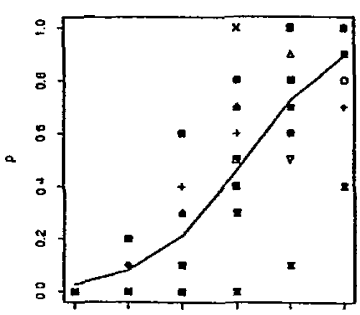

Isolado 498

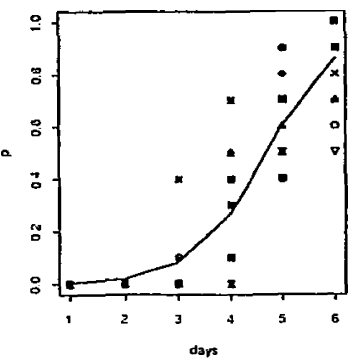

Isolado 462

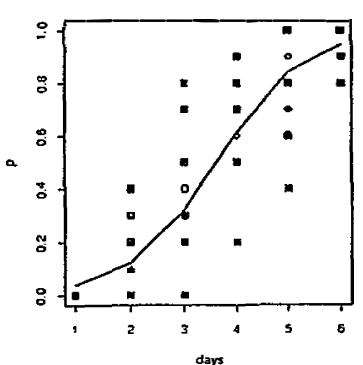

Isolado 520

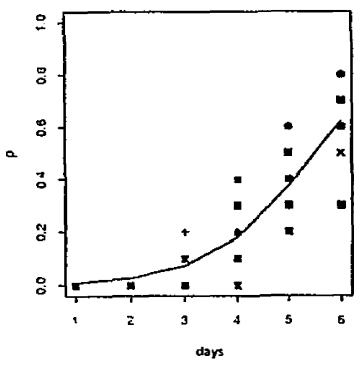

Isolado 868

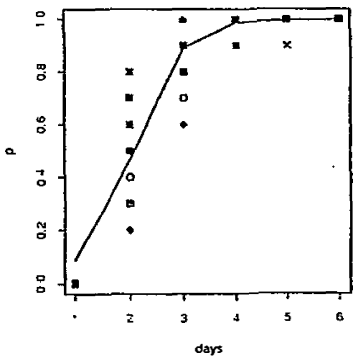

Isolado pl.61

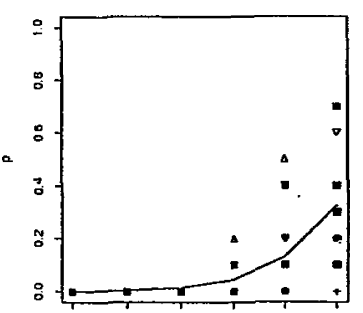

Isolado 499

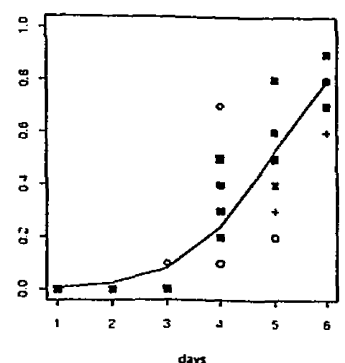

Isolado 522

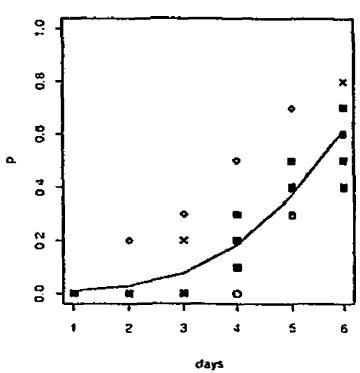

Isolado $\mathbf{5 2 9}$

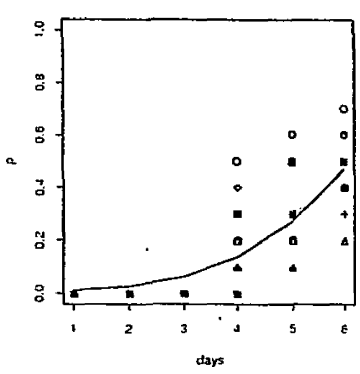

Isolado 1058

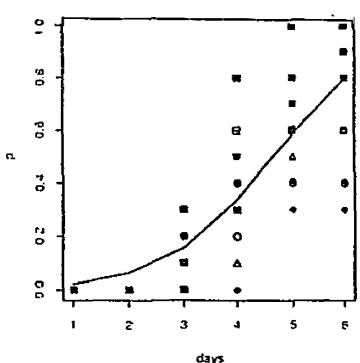

Isolado 641

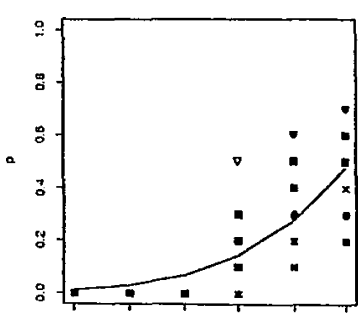

Figura 13: Proporção da mortalidade acumulada durante os 6 dias, para quinze isolados do experimento com o ácaro T. urticae. 


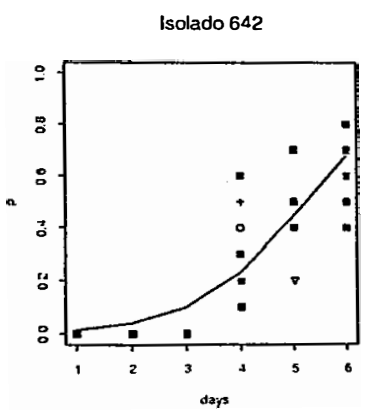

Isolado 457

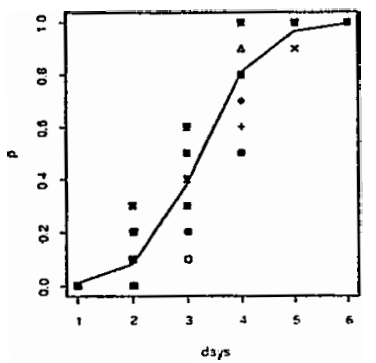

Isolado $6: 8$

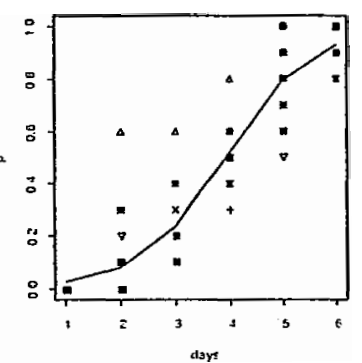

Isolado 268

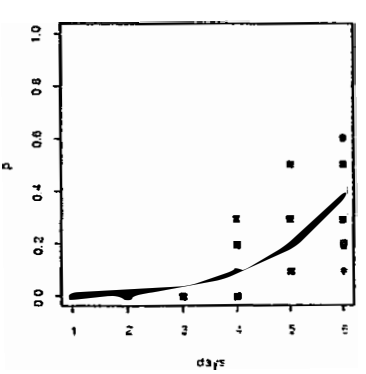

Isolado 501

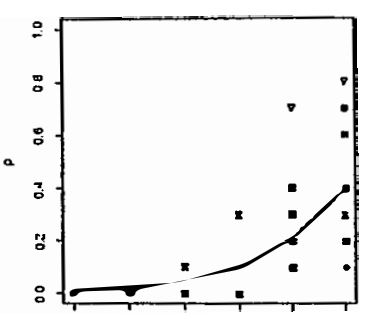

Isolado 722

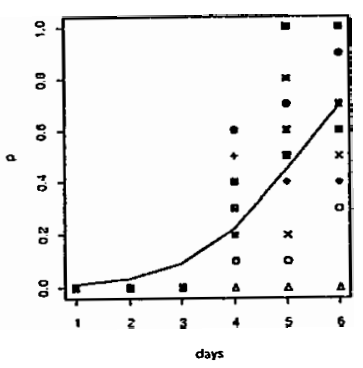

Isolado 485

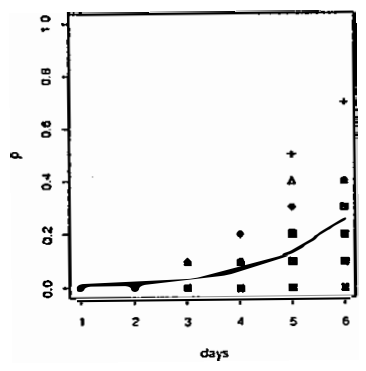

Isolado 620

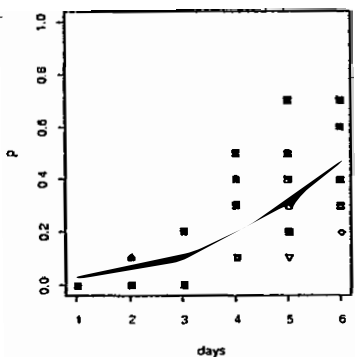

Isolado 309

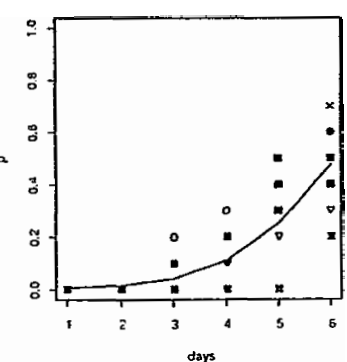

Isolado 695

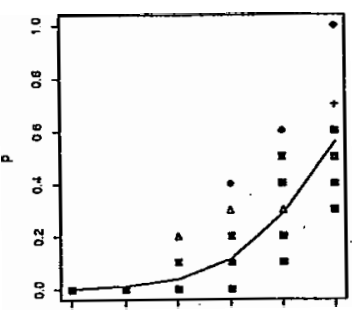

Isolado 307

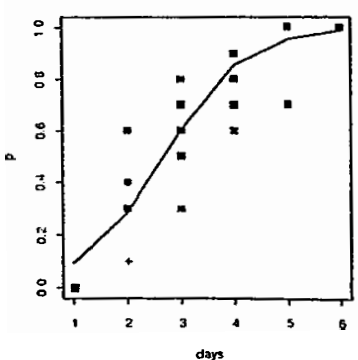

Isolado 489

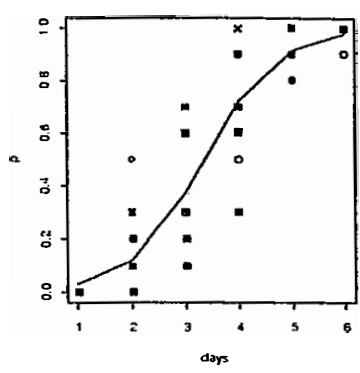

Isolado 787

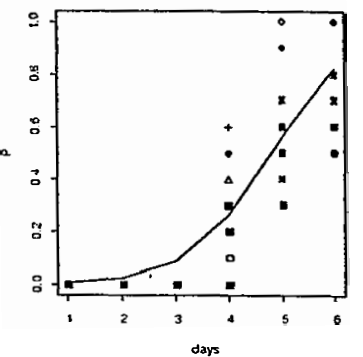

Isolado 498

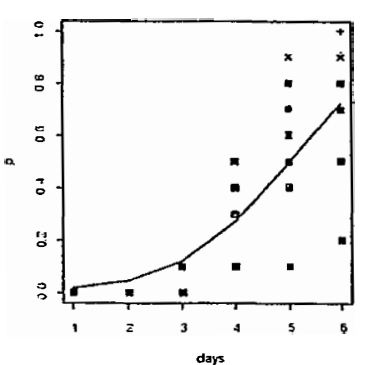

|solado pi-63

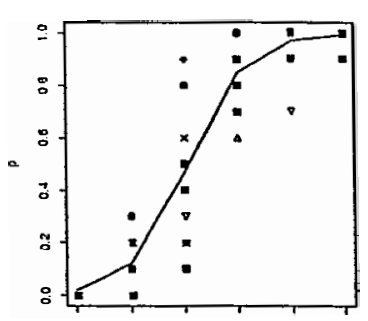

Figura 14: Proporção da mortalidade acumulada durante os 6 dias, para quinze isolados do experimento com o ácaro T. urticae. 

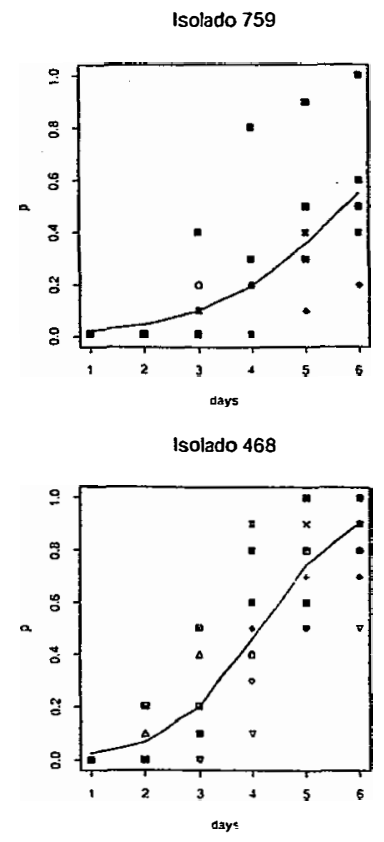

Isolado 755

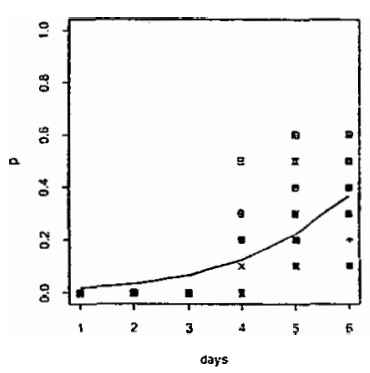

Isolado 573

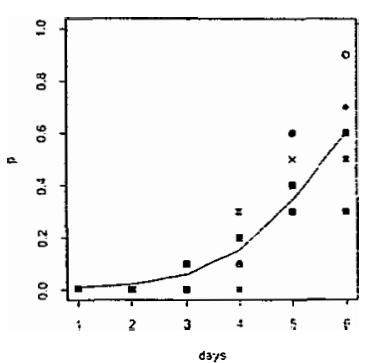

Isolado 615

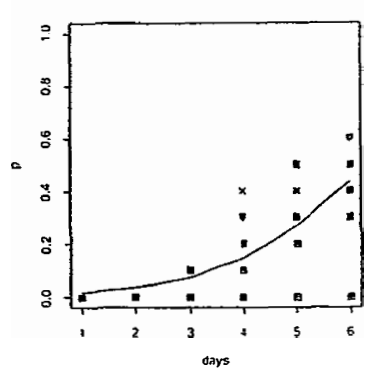

Isolado 532

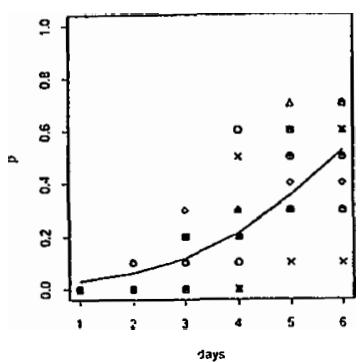

solado 759

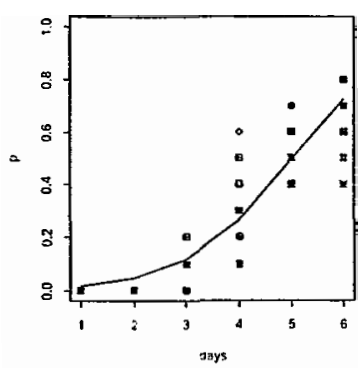

Isolado 1192

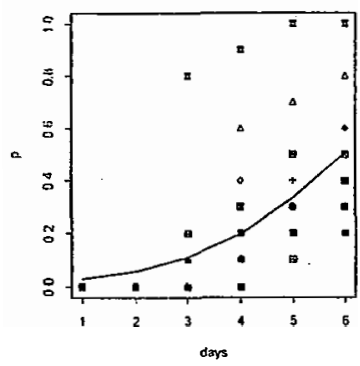

Isolado 579

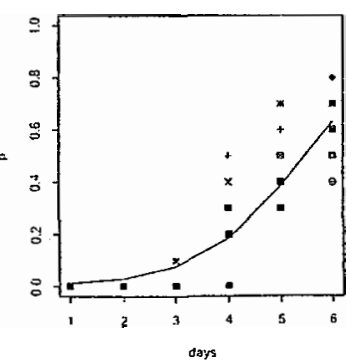

Isolado 1103

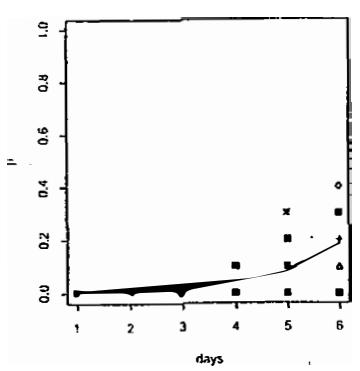

Isolado $\mathbf{2 1 0}$
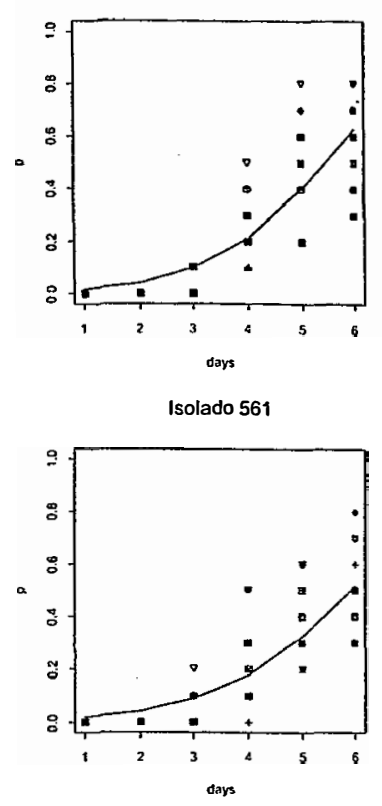

Isolado 569

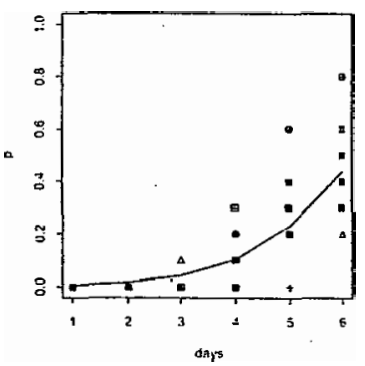

Isolado 590

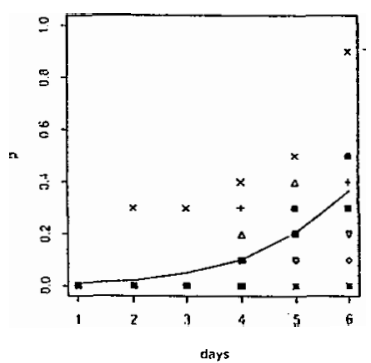

Isolado 619

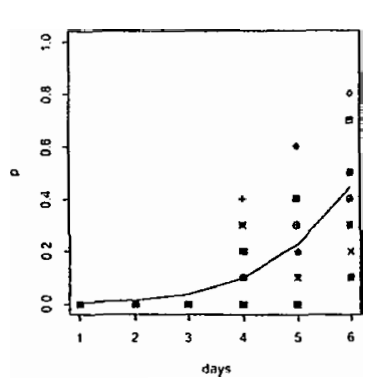

Figura 15: Proporção da mortalidade acumulada durante os 6 dias, para quinze isolados do experimento com o ácaro T. urticae. 


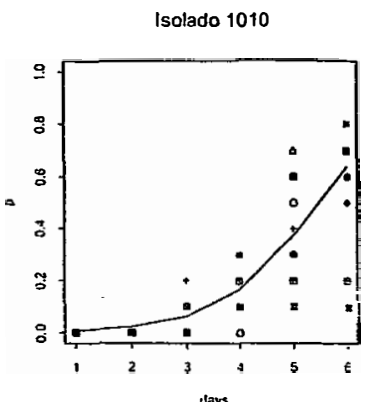

Isolado 647

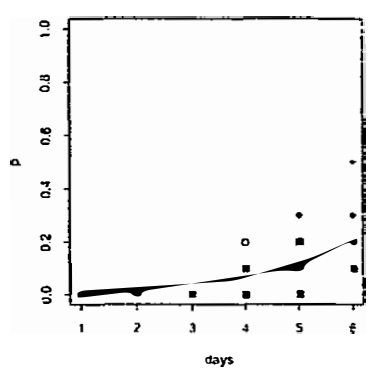

Isolado 1108

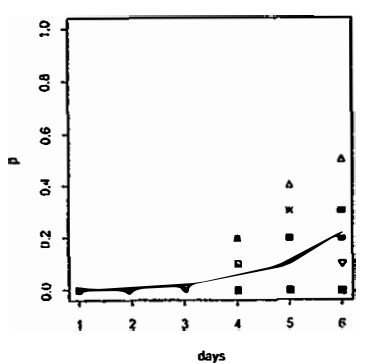

Isolado 739

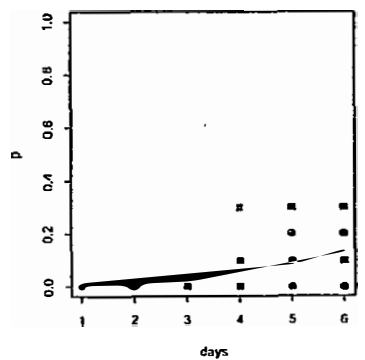

Isolado 644

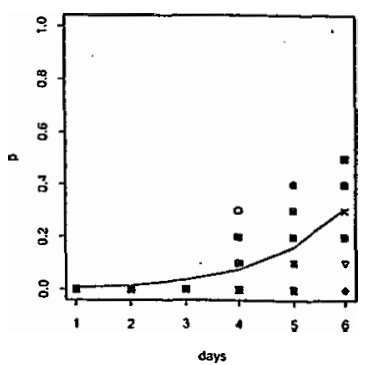

Figura 16: Proporção da mortalidade acumulada durante os 6 dias, para cinco isolados do experimento com o ácaro T. urticae. 
(a) Assintótica
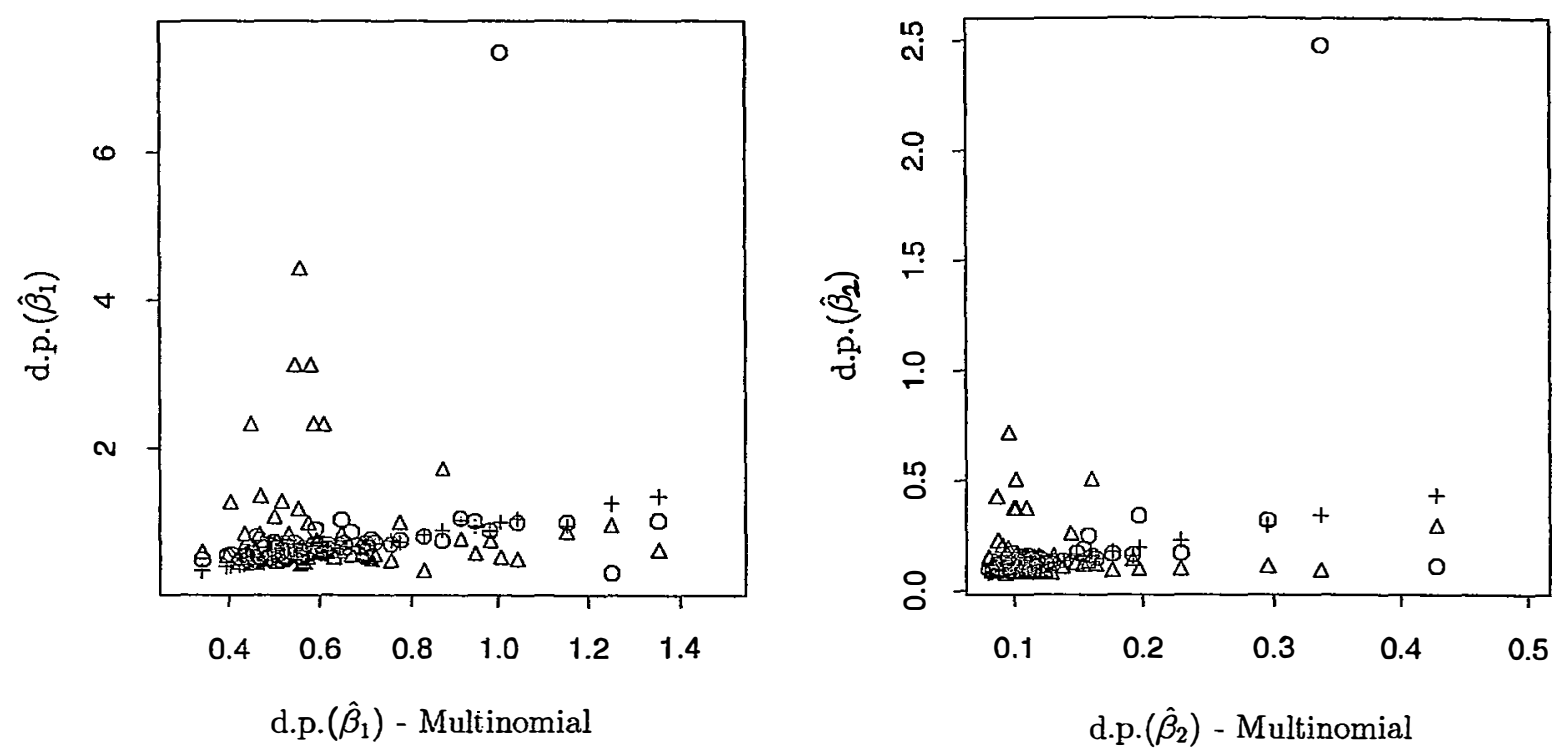

(b) Robusta
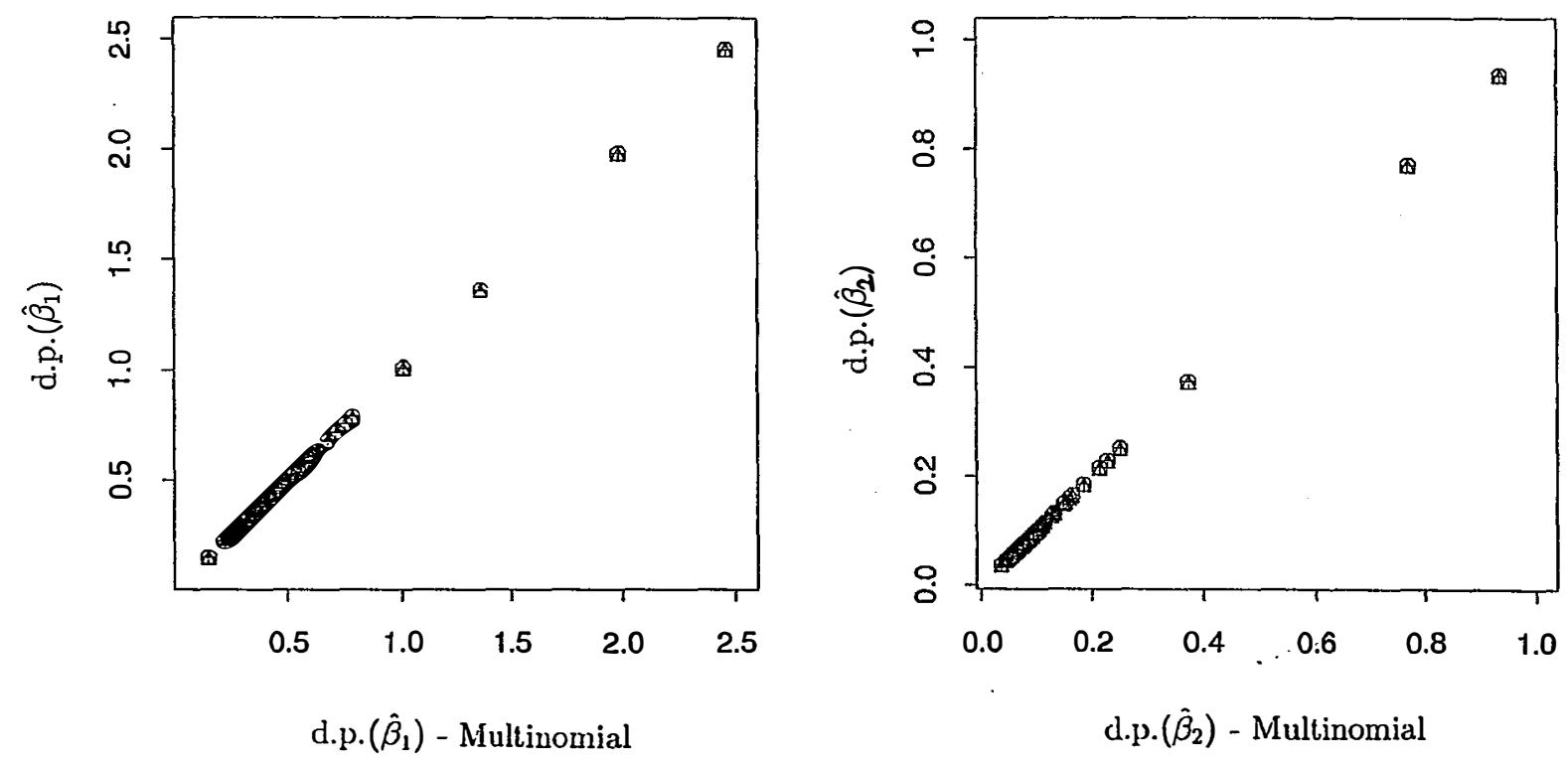

Figura 17: Diagramas de dispersão do d.p. $\left(\hat{\beta}_{1}\right)$ e d.p. $\left(\hat{\beta}_{2}\right)$ para o modelo multinomial $\times \mathrm{DM}(\mathrm{o})$, REMI $(\triangle)$ e REMII (+), usando a matriz de variâncias e covariância assintótica (a) e robusta (b). 
(a)

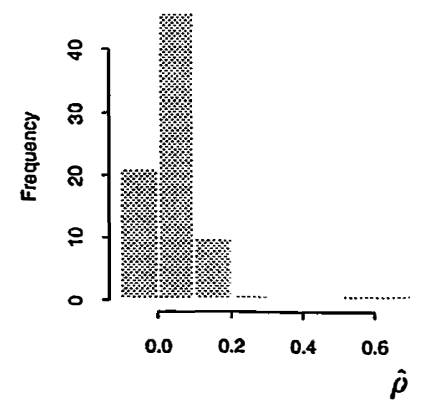

(d)

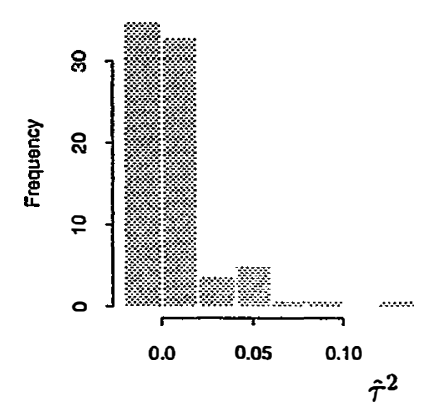

(f)

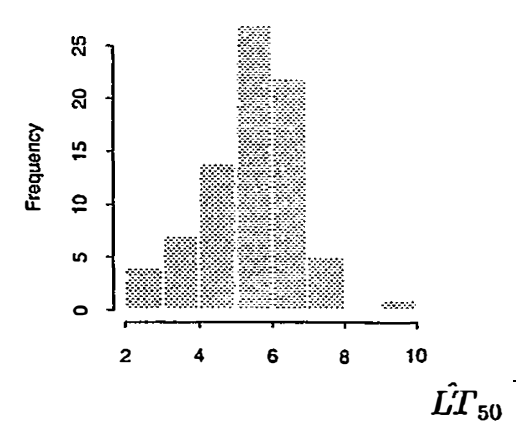

(h)

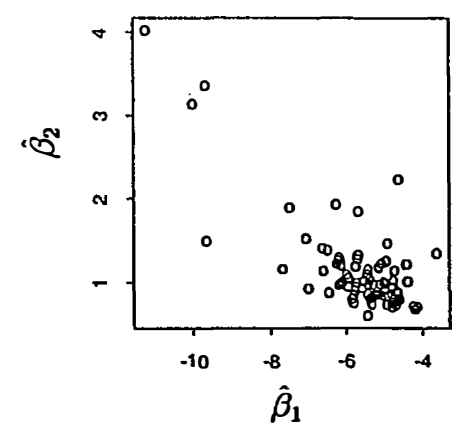

(b)

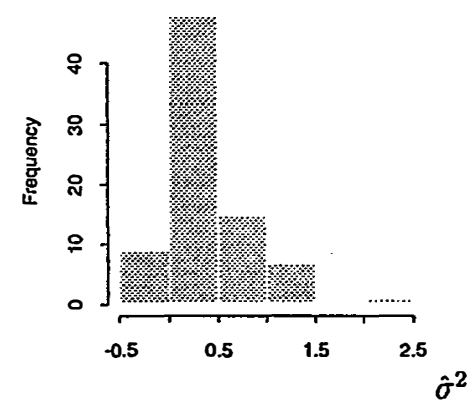

(e)

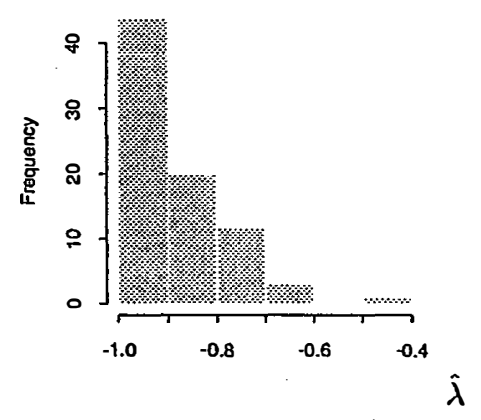

(g)

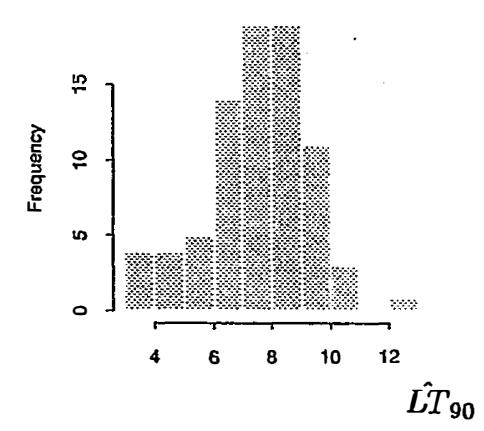

(h)

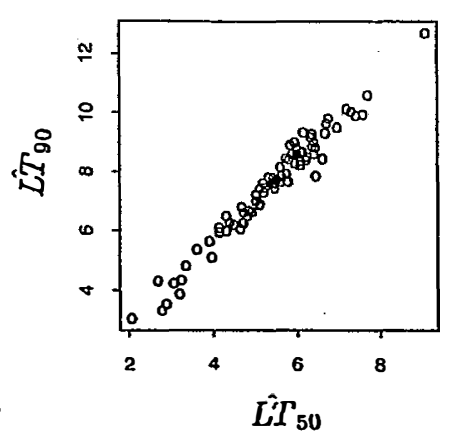

Figura 18: Histogramas de $\hat{\rho}, \hat{\sigma}^{2}, \hat{\tau}^{2}, \hat{\lambda}, \hat{L T}_{50}$ e $\hat{L T} \hat{T}_{00}$ e os diagramas de dispersão do $\hat{\beta}_{1} \times \hat{\beta}_{2}$ e $\hat{L T}_{50} \times \hat{L T}_{90}$. 
Tabela 11: Estimativas dos desvios padrões (d.p.) das estimativas dos parâmetros do modelo de regressão calculado através da matriz de variâncias e covariâncias assintótica, para o ensaio com os ácaros.

\begin{tabular}{|c|c|c|c|c|c|c|c|c|c|}
\hline \multirow[t]{2}{*}{ Isolado } & \multirow[t]{2}{*}{$i$} & \multicolumn{2}{|c|}{ multinomial } & \multicolumn{2}{|c|}{ Dirichlet-multinomial } & \multicolumn{2}{|c|}{ REMI } & \multicolumn{2}{|c|}{ REMI } \\
\hline & & d.p. $\left(\hat{\beta}_{1}\right)$ & d.p. $\left(\hat{\beta}_{2}\right)$ & d.p. $\left(\hat{\beta}_{1}\right)$ & d.p. $\left(\hat{\beta}_{2}\right)$ & d.p. $\left(\hat{\beta}_{1}\right)$ & d.p. $\left(\hat{\beta}_{2}\right)$ & d.p. $\left(\hat{\beta}_{1}\right)$ & d.p. $\left(\hat{\beta}_{2}\right)$ \\
\hline 756 & 1 & 0.6303 & 0.1084 & 0.5300 & 0.0912 & 0.5197 & 0.0925 & 0.6303 & 0.1084 \\
\hline 548 & 2 & 0.5296 & 0.0942 & 0.6691 & 0.1190 & 0.5459 & 0.0942 & 0.5197 & 0.0925 \\
\hline 549 & 3 & 0.6076 & 0.1084 & 0.6024 & 0.1074 & 0.6138 & 0.1084 & 0.6076 & 0.1084 \\
\hline 760 & 4 & 0.6171 & 0.1085 & 0.6978 & 0.1227 & 0.6585 & 0.1085 & 0.6171 & 0.1085 \\
\hline 551 & 5 & 0.4609 & 0.0991 & 0.8110 & 0.1744 & 0.6246 & 0.0991 & 0.4609 & 0.0991 \\
\hline 900 & 6 & 0.6518 & 0.1137 & 0.7210 & 0.1258 & 0.6519 & 0.1137 & 0.6518 & 0.1137 \\
\hline 600 & 7 & 0.6469 & 0.1580 & 1.0307 & 0.2516 & 0.8411 & 0.1367 & 0.6469 & 0.1580 \\
\hline 757 & 8 & 0.5910 & 0.1030 & 0.9107 & 0.1587 & 0.7213 & 0.1580 & 0.5910 & 0.1030 \\
\hline 1202 & 9 & 1.3547 & 0.2291 & 1.0209 & 0.1726 & 0.6290 & 0.1030 & 1.3547 & 0.2291 \\
\hline 635 & 10 & 0.4700 & 0.0873 & .0 .5223 & 0.0970 & 1.3563 & 0.2291 & 0.4700 & 0.0873 \\
\hline 749 & 11 & 0.5327 & 0.0981 & 0.6099 & 0.1123 & 0.8411 & 0.1367 & 0.5327 & 0.0981 \\
\hline 1197 & 12 & 0.5647 & 0.1018 & 0.6090 & 0.1098 & 0.4700 & 0.0873 & 0.5647 & 0.1018 \\
\hline 196 & 13 & 0.5027 & 0.0909 & 0.6019 & 0.1088 & 0.5914 & 0.1018 & 0.5027 & 0.0909 \\
\hline 252 & 14 & 0.4686 & 0.0891 & 0.6583 & 0.1252 & 0.8411 & 0.1367 & 0.4686 & 0.0891 \\
\hline 476 & 15 & 0.7105 & 0.1193 & 0.7709 & 0.1294 & 0.5027 & 0.0909 & 0.7105 & 0.1193 \\
\hline 494 & 16 & 1.0052 & 0.3379 & 7.3600 & 2.4744 & 0.5241 & 0.0891 & 1.0052 & 0.3379 \\
\hline 1006 & 17 & 0.5059 & 0.1075 & 0.6426 & 0.1366 & 0.7340 & 0.1193 & 0.5059 & 0.1075 \\
\hline 876 & 18 & 0.6018 & 0.1025 & 0.7062 & 0.1203 & 0.5794 & 0.1075 & 0.6018 & 0.1025 \\
\hline 890 . & 19 & 0.5164 & 0.0909 & 0.6279 & 0.1105 & 1.2829 & 0.2095 & 0.5164 & 0.0909 \\
\hline 893 & 20 & 0.6412 & 0.1105 & 0.6924 & 0.1194 & 0.6102 & 0.1025 & 0.6412 & 0.1105 \\
\hline 895 & 21 & 0.5626 & 0.0988 & 0.6583 & 0.1156 & 0.5170 & 0.0909 & 0.5626 & 0.0988 \\
\hline 881 & 22 & 0.5436 & 0.0939 & 0.7273 & 0.1257 & 0.6489 & 0.1105 & 0.5436 & 0.0939 \\
\hline 887 & 23 & 0.5837 & 0.1119 & 0.6248 & 0.1198 & 0.5849 & 0.0988 & 0.5837 & 0.1119 \\
\hline 612 & 24 & 0.6169 & 0.1289 & 0.6445 & 0.1347 & 0.5901 & 0.1119 & 0.6169 & 0.1289 \\
\hline 931 & 25 & 0.5756 & 0.1101 & 0.5740 & 0.1098 & 1.0017 & 0.1636 & 0.5756 & 0.1101 \\
\hline 764 & 26 & 0.5146 & 0.0996 & 0.5445 & 0.1054 & 0.6174 & 0.1289 & 0.5146 & 0.0996 \\
\hline 908 & 27 & 0.9470 & 0.2960 & 1.0152 & 0.3173 & 0.5812 & 0.1101 & 0.9470 & 0.2960 \\
\hline 986 & 28 & 0.5266 & 0.1048 & 0.4988 & 0.0993 & 0.5246 & 0.0996 & 0.5266 & 0.1048 \\
\hline 969 & 29 & 1.2517 & 0.4284 & 0.3127 & 0.1070 & 0.9579 & 0.2960 & 1.2517 & 0.4284 \\
\hline 987 & 30 & 0.5729 & 0.1181 & 0.5962 & 0.1229 & 0.5266 & 0.1048 & 0.5729 & 0.1181 \\
\hline 902 & 31 & 0.4040 & 0.0869 & 0.5661 & 0.1217 & 1.2732 & 0.4284 & 0.4040 & 0.0869 \\
\hline 498 & 32 & 0.5864 & 0.1203 & 0.6907 & 0.1417 & 0.6061 & 0.1181 & 0.5864 & 0.1203 \\
\hline 499 & 33 & 0.5660 & 0.1116 & 0.5590 & 0.1103 & 0.4672 & 0.0869 & 0.5660 & 0.1116 \\
\hline 500 & 34 & 0.4955 & 0.1006 & 0.6160 & 0.1251 & 0.6037 & 0.1203 & 0.4955 & 0.1006 \\
\hline 462 & 35 & 0.3951 & 0.0996 & 0.5508 & 0.1389 & 0.5498 & 0.1006 & 0.3951 & 0.0996 \\
\hline 522 & 36 & 0.5532 & 0.1007 & 0.5443 & 0.0991 & 1.1872 & 0.1650 & 0.5532 & 0.1007 \\
\hline
\end{tabular}


Continuação da Tabela (11). Estimativas dos desvios padrões (d.p.) das estimativas dos parâmetros do modelo de regressão calculado através da matriz de variâncias e covariâncias assintótica, para o ensaio com os ácaros.

\begin{tabular}{|c|c|c|c|c|c|c|c|c|c|}
\hline \multirow[t]{2}{*}{ Isolado } & \multirow[t]{2}{*}{$i$} & \multicolumn{2}{|c|}{ multinomial } & \multicolumn{2}{|c|}{ Dirichlet-multinomial } & \multicolumn{2}{|c|}{ REMI } & \multicolumn{2}{|c|}{ REMII } \\
\hline & & d.p. $\left(\hat{\beta}_{1}\right)$ & d.p. $\left(\hat{\beta}_{2}\right)$ & d.p. $\left(\hat{\beta}_{1}\right)$ & d.p. $\left(\hat{\beta}_{2}\right)$ & d.p. $\left(\hat{\beta}_{1}\right)$ & d.p. $\left(\hat{\beta}_{2}\right)$ & d.p. $\left(\hat{\beta}_{1}\right)$ & d.p. $\left(\hat{\beta}_{2}\right)$ \\
\hline 589 & 37 & 0.4624 & 0.0871 & 0.5149 & 0.0970 & 0.4488 & 0.0996 & 0.4624 & 0.0871 \\
\hline $\mathbf{5 2 0}$ & 38 & 0.5634 & 0.1028 & 0.5138 & 0.0937 & 0.4654 & 0.0871 & 0.5634 & 0.1028 \\
\hline 529 & 39 & 0.5943 & 0.1037 & 0.6868 & 0.1198 & 0.7756 & 0.1030 & 0.5943 & 0.1037 \\
\hline 643 & 40 & 0.4995 & 0.1101 & 0.7361 & 0.1622 & 0.5640 & 0.1028 & 0.4995 & 0.1101 \\
\hline 868 & 41 & 0.4404 & 0.1975 & 0.6661 & 0.3410 & 0.6047 & 0.1037 & 0.4404 & 0.1975 \\
\hline 1058 & 42 & 0.4437 & 0.0901 & 0.5855 & 0.1189 & 0.5728 & 0.1101 & 0.4437 & 0.0901 \\
\hline 1078 & 43 & 0.4237 & 0.0950 & 0.4981 & 0.1116 & 0.4579 & 0.1975 & 0.4237 & 0.0950 \\
\hline pl61 & 44 & 1.0437 & 0.1768 & 1.0014 & 0.1696 & 0.5053 & 0.0950 & 1.0437 & 0.1768 \\
\hline 641 & 45 & 0.5803 & 0.1014 & 0.6344 & 0.1109 & 3.1341 & 0.5096 & 0.5803 & 0.1014 \\
\hline 642 & 46 & 0.5008 & 0.0941 & 0.6445 & 0.1211 & 1.0780 & 0.1768 & 0.5008 & 0.0941 \\
\hline 722 & 47 & 0.5239 & 0.0987 & 0.7149 & 0.1348 & 0.5925 & 0.1014 & 0.5239 & 0.0987 \\
\hline 307 & 48 & 0.3422 & 0.1117 & 0.4944 & 0.1613 & 0.5978 & 0.0987 & 0.3422 & 0.1117 \\
\hline 457 & 49 & 0.5462 & 0.1606 & 0.5478 & 0.1611 & 3.1341 & 0.5096 & 0.5462 & 0.1606 \\
\hline 485 & 50 & 0.8272 & 0.1384 & 0.8109 & 0.1357 & 0.3439 & 0.1117 & 0.8272 & 0.1384 \\
\hline 489 & 51 & 0.4319 & 0.1192 & 0.5438 & 0.1501 & 0.5616 & 0.1606 & 0.4319 & 0.1192 \\
\hline 618 & 52 & 0.4355 & 0.1032 & 0.6113 & 0.1449 & 0.8570 & 0.1384 & 0.4355 & 0.1032 \\
\hline 620 & 53 & 0.4754 & 0.0830 & 0.5577 & 0.0974 & 0.4796 & 0.1192 & 0.4754 & 0.0830 \\
\hline 787 & 54 & 0.5579 & 0.1121 & 0.6220 & 0.1250 & 0.4400 & 0.1032 & 0.5579 & 0.1121 \\
\hline 268 & 55 & 0.7533 & 0.1292 & 0.7043 & 0.1208 & 0.4868 & 0.0830 & 0.7533 & 0.1292 \\
\hline 309 & 56 & 0.6897 & 0.1202 & 0.6542 & 0.1140 & 0.5872 & 0.1121 & 0.6897 & 0.1202 \\
\hline 498 & 57 & 0.4761 & 0.0918 & 0.5671 & 0.1094 & 0.7581 & 0.1292 & 0.4761 & 0.0918 \\
\hline 501 & 58 & 0.6971 & 0.1196 & 0.7158 & 0.1228 & 0.6924 & 0.1202 & 0.6971 & 0.1196 \\
\hline 695 & 59 & 0.7034 & 0.1247 & 0.6575 & 0.1166 & 0.5262 & 0.0918 & 0.7034 & 0.1247 \\
\hline pl63 & 60 & 0.5004 & 0.1539 & 0.6208 & 0.1909 & 0.7184 & 0.1196 & 0.5004 & 0.1539 \\
\hline 759 & 61 & 0.4927 & 0.0882 & 0.5620 & 0.1006 & 0.7133 & 0.1247 & 0.4927 & 0.0882 \\
\hline 532 & 62 & 0.4552 & 0.0809 & 0.5464 & 0.0971 & 0.5372 & 0.1539 & 0.4552 & 0.0809 \\
\hline 210 & 63 & 0.5049 & 0.0928 & 0.5489 & 0.1009 & 0.4696 & 0.0809 & 0.5049 & 0.0928 \\
\hline 468 & 64 & 0.4489 & 0.1015 & 0.5886 & 0.1331 & 2.3452 & 0.3789 & 0.4489 & 0.1015 \\
\hline 759 & 65 & 0.4809 & 0.0927 & 0.5002 & 0.0964 & 0.5112 & 0.0928 & 0.4809 & 0.0927 \\
\hline 561 & 66 & 0.5168 & 0.0915 & 0.5763 & 0.1020 & 0.4809 & 0.0927 & 0.5168 & 0.0915 \\
\hline 755 & 67. & 0.5862 & 0.0997 & 0.7122 & 0.1211 & 2.3452 & 0.3789 & 0.5862 & 0.0997 \\
\hline 1192 & 68 & 0.4707 & 0.0831 & 0.7666 & 0.1353 & 0.5206 & 0.0915 & 0.4707 & 0.0831 \\
\hline 569 & 69 & 0.6958 & 0.1203 & 0.6685 & 0.1156 & 0.5513 & 0.0831 & 0.6958 & 0.1203 \\
\hline 573 & 70 & 0.6094 & 0.1100 & 0.5610 & 0.1013 & 2.3452 & 0.3789 & 0.6094 & 0.1100 \\
\hline 579 & 71 & 0.5592 & 0.1024 & 0.5422 & 0.0993 & 0.6982 & 0.1203 & 0.5592 & 0.1024 \\
\hline 590 & 72 & 0.6667 & 0.1136 & 0.8708 & 0.1484 & 0.5592 & 0.1024 & 0.6667 & 0.1136 \\
\hline
\end{tabular}


Continuação da Tabela (11). Estimativas dos desvios padrões (d.p.) das estimativas dos parâmetros do modelo de regressão calculado através da matriz de variâncias e covariâncias assintótica, para o ensaio com os ácaros.

\begin{tabular}{c|c|cc|cc|cc|cc}
\hline Isolado & $i$ & \multicolumn{2}{|c|}{ multinomial } & \multicolumn{2}{c|}{ Dirichlet-multinomial } & \multicolumn{2}{c}{ REMI } & \multicolumn{2}{c}{ REMII } \\
& & d.p. $\left(\hat{\beta}_{1}\right)$ & d.p. $\left(\hat{\beta}_{2}\right)$ & d.p. $\left(\hat{\beta}_{1}\right)$ & d.p. $\left(\hat{\beta}_{2}\right)$ & d.p. $\left(\hat{\beta}_{1}\right)$ & d.p. $\left(\hat{\beta}_{2}\right)$ & d.p. $\left(\hat{\beta}_{1}\right)$ & d.p. $\left(\hat{\beta}_{2}\right)$ \\
\hline 615 & 73 & 0.5562 & 0.0962 & 0.5486 & 0.0949 & 4.4329 & 0.7203 & 0.5562 & 0.0962 \\
1103 & 74 & 1.1550 & 0.1922 & 0.9947 & 0.1656 & 0.8696 & 0.1445 & 0.9481 & 0.1581 \\
619 & 75 & 0.7175 & 0.1241 & 0.7313 & 0.1265 & 0.5607 & 0.0962 & 0.7010 & 0.1205 \\
1010 & 76 & 0.5987 & 0.1095 & 0.6417 & 0.1174 & 0.6612 & 0.1107 & 0.6268 & 0.1165 \\
1108 & 77 & 0.9817 & 0.1639 & 0.8859 & 0.1479 & 0.7502 & 0.1241 & 0.8806 & 0.1474 \\
644 & 78 & 0.7740 & 0.1306 & 0.7527 & 0.1270 & 0.9975 & 0.1639 & 0.7217 & 0.1222 \\
647 & 79 & 0.8696 & 0.1445 & 0.7536 & 0.1252 & 1.7360 & 0.2641 & 0.8904 & 0.1487 \\
739 & 80 & 0.9129 & 0.1488 & 1.0580 & 0.1725 & 0.7817 & 0.1306 & 1.0309 & 0.1687 \\
\hline
\end{tabular}


Tabela 12: Estimativas dos desvios padrões (d.p.) das estimativas dos parâmetros do modelo de regressão calculado através da matriz de variâncias e covariâncias robusta, para o ensaio com os ácaros.

\begin{tabular}{|c|c|c|c|c|c|c|c|c|c|}
\hline \multirow[t]{2}{*}{ Isolado } & \multirow[t]{2}{*}{$i$} & \multicolumn{2}{|c|}{ multinomial } & \multicolumn{2}{|c|}{ Dirichlet-multinomial } & \multicolumn{2}{|c|}{ REMI } & \multicolumn{2}{|c|}{ REMII } \\
\hline & & d.p. $\left(\hat{\beta}_{1}\right)$ & d.p. $\left(\hat{\beta}_{2}\right)$ & d.p. $\left(\hat{\beta}_{1}\right)$ & d.p. $\left(\hat{\beta}_{2}\right)$ & d.p. $\left(\hat{\beta}_{1}\right)$ & d.p. $\left(\hat{\beta}_{2}\right)$ & d.p. $\left(\hat{\beta}_{1}\right)$ & d.p. $\left(\hat{\beta}_{2}\right)$ \\
\hline 756 & 1 & 0,2778 & 0,0526 & 0,2778 & 0,0526 & 0,2778 & 0,0526 & 0,2778 & 0,0526 \\
\hline 548 & 2 & 0,2789 & 0,0690 & 0,2789 & 0,0690 & 0,2789 & 0,0690 & 0,2789 & 0,0690 \\
\hline 549 & 3 & 0,4459 & 0,0950 & 0,4459 & 0,0950 & 0,4459 & 0,0950 & 0,4459 & 0,0950 \\
\hline 760 & 4 & 0,2727 & 0,0810 & 0,2727 & 0,0810 & 0,2727 & 0,0810 & 0,2727 & 0,0810 \\
\hline 551 & 5 & 0,5276 & 0,1633 & 0,5276 & 0,1633 & 0,5276 & 0,1633 & 0,5276 & 0,1633 \\
\hline 900 & 6 & 0,5166 & 0,1102 & 0,5166 & 0,1102 & 0,5166 & 0,1102 & 0,5166 & 0,1102 \\
\hline 600 & 7 & 0,7515 & 0,2510 & 0,7515 & 0,2510 & 0,7515 & 0,2510 & 0,7515 & 0,2510 \\
\hline 757 & 8 & 0,5640 & 0,0814 & 0,5640 & 0,0814 & 0,5640 & 0,0814 & 0,5640 & 0,0814 \\
\hline 1202 & 9 & 1,3582 & 0,2282 & 1,3582 & 0,2282 & 1,3582 & 0,2282 & 1,3582 & 0,2282 \\
\hline 635 & 10 & 0,1477 & 0,0463 & 0,1477 & 0,0463 & 0,1477 & 0,0463 & 0,1477 & 0,0463 \\
\hline 749 & 11 & 0,2619 & 0,0520 & 0,2619 & 0,0520 & 0,2619 & 0,0520 & 0,2619 & 0,0520 \\
\hline 1197 & 12 & 0,2326 & 0,0732 & 0,2326 & 0,0732 & 0,2326 & 0,0732 & 0,2326 & 0,0732 \\
\hline 196 & 13 & 0,4322 & 0,0754 & 0,4322 & 0,0754 & 0,4322 & 0,0754 & 0,4322 & 0,0754 \\
\hline 252 & 14 & 0,3125 & 0,0889 & 0,3125 & 0,0889 & 0,3125 & 0,0889 & 0,3125 & 0,0889 \\
\hline 476 & 15 & 0,6170 & 0,0965 & 0,6170 & 0,0965 & 0,6170 & 0,0965 & 0,6170 & 0,0965 \\
\hline 494 & 16 & 1,9817 & 0,7686 & 1,9817 & 0,7686 & 1,9817 & 0,7686 & 1,9817 & 0,7686 \\
\hline 1006 & 17 & 0,4488 & 0,1293 & 0,4488 & 0,1293 & 0,4488 & 0,1293 & 0,4488 & 0,1293 \\
\hline 876 & 18 & 0,3769 & 0,0471 & 0,3769 & 0,0471 & 0,3769 & 0,0471 & 0,3769 & 0,0471 \\
\hline 890 & 19 & 0,2855 & 0,0597 & 0,2855 & 0,0597 & 0,2855 & 0,0597 & 0,2855 & 0,0597 \\
\hline 893 & 20 & 0,4372 & 0,0854 & 0,4372 & 0,0854 & 0,4372 & 0,0854 & 0,4372 & 0,0854 \\
\hline 895 & 21 & 0,3842 & 0,0648 & 0,3842 & 0,0648 & 0,3842 & 0,0648 & 0,3842 & 0,0648 \\
\hline 881 & 22 & 0,2864 & 0,0452 & 0,2864 & 0,0452 & 0,2864 & 0,0452 & 0,2864 & 0,0452 \\
\hline 887 & 23 & 0,3968 & 0,0994 & 0,3968 & 0,0994 & 0,3968 & 0,0994 & 0,3968 & 0,0994 \\
\hline 612 & 34 & 0,7141 & 0,1582 & 0,7141 & 0,1582 & 0,7141 & 0,1582 & 0,7141 & 0,1582 \\
\hline 931 & 25 & 0,4071 & 0,0968 & 0,4071 & 0,0968 & 0,4071 & 0,0968 & 0,4071 & 0,0968 \\
\hline 764 & 26 & 0,4045 & 0,0941 & 0,4045 & 0,0941 & 0,4045 & 0,0941 & 0,4045 & 0,0941 \\
\hline 908 & 27 & 1,0073 & 0,3740 & 1,0073 & 0,3740 & 1,0073 & 0,3740 & 1,0073 & 0,3740 \\
\hline 986 & 28 & $0, \mathbf{3 1 7 0}$ & 0,0615 & 0,3170 & 0,0615 & $0, \mathbf{3 1 7 0}$ & 0,0615 & 0,3170 & 0,0615 \\
\hline 969 & 29 & 2,4525 & 0,9337 & 2,4525 & 0,9337 & 2,4525 & 0,9337 & 2,4525 & 0,9337 \\
\hline 987 & 30 & 0,3830 & 0,0976 & $\mathbf{0 , 3 8 3 0}$ & 0,0976 & 0,3830 & 0,0976 & 0,3830 & 0,0976 \\
\hline 902 & 31 & 0,3516 & 0,0981 & 0,3516 & 0,0981 & 0,3516 & 0,0981 & 0,3516 & 0,0981 \\
\hline 498 & 32 & 0,6765 & 0,1473 & 0,6765 & 0,1473 & 0,6765 & 0,1473 & 0,6765 & 0,1473 \\
\hline 499 & 33 & 0,4138 & 0,0794 & 0,4138 & 0,0794 & 0,4138 & 0,0794 & 0,4138 & 0,0794 \\
\hline 500 & 34 & 0,4116 & 0,0998 & 0,4116 & 0,0998 & 0,4116 & 0,0998 & 0,4116 & 0,0998 \\
\hline 462 & 35 & 0,5198 & 0,1135 & 0,5198 & 0,1135 & 0,5198 & 0,1135 & 0,5198 & 0,1135 \\
\hline 522 & 36 & 0,5565 & 0,0973 & 0,5565 & 0,0973 & 0,5565 & 0,0973 & 0,5565 & 0,0973 \\
\hline
\end{tabular}


Continuação da Tabela(12). Estimativas dos desvios padrões (d.p.) das estimativas dos parâmetros do modelo de regressão calculado através da matriz de variâncias e covariâncias robusta, para o ensaio com os ácaros.

\begin{tabular}{|c|c|c|c|c|c|c|c|c|c|}
\hline \multirow[t]{2}{*}{ Isolado } & \multirow[t]{2}{*}{$i$} & \multicolumn{2}{|c|}{ multinomial } & \multicolumn{2}{|c|}{ Dirichlet-multinomial } & \multicolumn{2}{|c|}{ REMI } & \multicolumn{2}{|c|}{ REMII } \\
\hline & & d.p. $\left(\hat{\beta}_{1}\right)$ & d.p. $\left(\hat{\beta}_{2}\right)$ & d.p. $\left(\hat{\beta}_{1}\right)$ & d.p. $\left(\hat{\beta}_{2}\right)$ & d.p. $\left(\hat{\beta}_{1}\right)$ & d.p. $\left(\hat{\beta}_{2}\right)$ & d.p. $\left(\hat{\beta}_{1}\right)$ & d.p. $\left(\hat{\beta}_{2}\right)$ \\
\hline 589 & 37 & 0,2328 & 0,0439 & 0,2328 & 0,0439 & 0,2328 & 0,0439 & 0,2328 & 0,0439 \\
\hline 520 & 38 & 0,3737 & 0,0703 & 0,3737 & 0,0703 & 0,3737 & 0,0703 & 0,3737 & 0,0703 \\
\hline 529 & 39 & 0,3984 & 0,0625 & 0,3984 & 0,0625 & 0,3984 & 0,0625 & 0,3984 & 0,0625 \\
\hline 643 & 40 & 0,6307 & 0,1843 & 0,6307 & 0,1843 & 0,6307 & 0,1843 & 0,6307 & 0,1843 \\
\hline 868 & 41 & 0,3437 & 0,2147 & $\mathbf{0 , 3 4 3 7}$ & 0,2147 & 0,3437 & 0,2147 & 0,3437 & 0,2147 \\
\hline 1058 & 42 & 0,3683 & 0,1011 & 0,3683 & 0,1011 & 0,3683 & 0,1011 & 0,3683 & 0,1011 \\
\hline 1078 & 43 & 0,4707 & 0,1146 & 0,4707 & 0,1146 & 0,4707 & 0,1146 & 0,4707 & 0,1146 \\
\hline pl44 & 53 & 0,7848 & 0,1293 & 0,7848 & 0,1293 & 0,7848 & 0,1293 & 0,7848 & 0,1293 \\
\hline 641 & 45 & 0,2824 & 0,0553 & 0,2824 & 0,0553 & 0,2824 & 0,0553 & 0,2824 & 0,0553 \\
\hline 642 & 46 & 0,2745 & 0,0435 & 0,2745 & 0,0435 & 0,2745 & 0,0435 & 0,2745 & 0,0435 \\
\hline 722 & 47 & 0,2585 & 0,1060 & 0,2585 & 0,1060 & 0,2585 & 0,1060 & 0,2585 & 0,1060 \\
\hline 307 & 48 & 0,2391 & 0,0831 & 0,2391 & 0,0831 & 0,2391 & 0,0831 & 0,2391 & 0,0831 \\
\hline 457 & 49 & 0,4962 & 0,1315 & 0,4962 & 0,1315 & 0,4962 & 0,1315 & 0,4962 & 0,1315 \\
\hline 485 & 50 & 0,4589 & 0,0691 & 0,4589 & 0,0691 & 0,4589 & 0,0691 & 0,4589 & 0,0691 \\
\hline 489 & 51 & 0,4867 & 0,1147 & 0,4867 & 0,1147 & 0,4867 & 0,1147 & 0,4867 & 0,1147 \\
\hline 618 & 52 & 0,5827 & 0,1262 & 0,5827 & 0,1262 & 0,5827 & 0,1262 & 0,5827 & 0,1262 \\
\hline 620 & 53 & 0,2493 & 0,0508 & 0,2493 & 0,0508 & 0,2493 & 0,0508 & 0,2493 & 0,0508 \\
\hline 787 & 54 & 0,2670 & 0,0885 & 0,2670 & 0,0885 & 0,2670 & 0,0885 & 0,2670 & 0,0885 \\
\hline 268 & 55 & 0,3924 & 0,0583 & 0,3924 & 0,0583 & 0,3924 & 0,0583 & 0,3924 & 0,0583 \\
\hline 309 & 56 & 0,4911 & 0,0711 & 0,4911 & 0,0711 & 0,4911 & 0,0711 & 0,4911 & 0,0711 \\
\hline 498 & 57 & 0,2197 & 0,0908 & 0,2197 & 0,0908 & 0,2197 & 0,0908 & 0,2197 & 0,0908 \\
\hline 501 & 58 & 0,5437 & 0,1066 & 0,5437 & 0,1066 & 0,5437 & 0,1066 & 0,5437 & 0,1066 \\
\hline 695 & 59 & 0,6072 & 0,1074 & 0,6072 & 0,1074 & 0,6072 & 0,1074 & 0,6072 & 0,1074 \\
\hline pl60 & 70 & 0,4336 & 0,1516 & 0,4336 & 0,1516 & 0,4336 & 0,1516 & 0,4336 & 0,1516 \\
\hline 759 & 61 & 0,3545 & 0,0504 & 0,3545 & 0,0504 & 0,3545 & 0,0504 & 0,3545 & 0,0504 \\
\hline 532 & 62 & 0,3231 & 0,0638 & 0,3231 & 0,0638 & 0,3231 & 0,0638 & 0,3231 & 0,0638 \\
\hline 210 & 63 & 0,2625 & 0,0711 & 0,2625 & 0,0711 & 0,2625 & 0,0711 & 0,2625 & 0,0711 \\
\hline 468 & 64 & 0,2883 & 0,0952 & 0,2883 & 0,0952 & 0,2883 & 0,0952 & 0,2883 & 0,0952 \\
\hline 759 & 65 & 0,2365 & 0,0483 & 0,2365 & 0,0483 & 0,2365 & 0,0483 & 0,2365 & 0,0483 \\
\hline 561 & 66 & 0,3638 & 0,0696 & 0,3638 & 0,0696 & 0,3638 & 0,0696 & 0,3638 & 0,0696 \\
\hline 755 & 67 & 0,2407 & 0,0362 & 0,2407 & 0,0362 & 0,2407 & 0,0362 & 0,2407 & 0,0362 \\
\hline 1192 & 68 & 0,5236 & 0,0622 & 0,5236 & 0,0622 & 0,5236 & 0,0622 & 0,5236 & 0,0622 \\
\hline 569 & 69 & 0,4626 & 0,0896 & 0,4626 & 0,0896 & 0,4626 & 0,0896 & 0,4626 & 0,0896 \\
\hline 573 & 70 & 0,4221 & 0,0936 & 0,4221 & 0,0936 & 0,4221 & 0,0936 & 0,4221 & 0,0936 \\
\hline 579 & 71 & 0,4372 & 0,0790 & 0,4372 & 0,0790 & 0,4372 & 0,0790 & 0,4372 & 0,0790 \\
\hline 590 & 72 & 0,5495 & 0,0766 & 0,5495 & 0,0766 & 0,5495 & 0,0766 & 0,5495 & 0,0766 \\
\hline
\end{tabular}


Continuação da Tabela (12). Estimativas dos desvios padrões (d.p.) das estimativas dos parâmetros do modelo de regressão calculado através da matriz de variâncias e covariâncias robusta, para o ensaio com os ácaros.

\begin{tabular}{|c|c|c|c|c|c|c|c|c|c|}
\hline \multirow[t]{2}{*}{ Isolado } & \multirow[t]{2}{*}{$i$} & \multicolumn{2}{|c|}{ multinomial } & \multicolumn{2}{|c|}{ Dirichlet-multinomial } & \multicolumn{2}{|c|}{ REMI } & \multicolumn{2}{|c|}{ REMII } \\
\hline & & d.p. $\left(\hat{\beta}_{1}\right)$ & d.p. $\left(\hat{\beta}_{2}\right)$ & d.p. $\left(\hat{\beta}_{1}\right)$ & d.p. $\left(\hat{\beta}_{2}\right)$ & d.p. $\left(\hat{\beta}_{1}\right)$ & d.p. $\left(\hat{\beta}_{2}\right)$ & d.p. $\left(\hat{\beta}_{1}\right)$ & d.p. $\left(\hat{\beta}_{2}\right)$ \\
\hline 615 & 73 & 0,3177 & 0,0572 & 0,3177 & 0,0572 & 0,3177 & 0,0572 & $\mathbf{0 , 3 1 7 7}$ & 0,0572 \\
\hline 1103 & 74 & 0,5743 & 0,1072 & 0,5743 & 0,1072 & 0,5743 & 0,1072 & 0,5743 & 0,1072 \\
\hline 619 & 75 & 0,4507 & 0,0629 & 0,4507 & 0,0629 & 0,4507 & 0,0629 & 0,4507 & 0,0629 \\
\hline 1010 & 76 & 0,4208 & 0,1023 & 0,4208 & 0,1023 & 0,4208 & 0,1023 & 0,4208 & 0,1023 \\
\hline 1108 & 77 & 0,6253 & 0,0974 & 0,6253 & 0,0974 & 0,6253 & 0,0974 & 0,6253 & 0,0974 \\
\hline 644 & 78 & 0,5253 & 0,0881 & 0,5253 & 0,0881 & 0,5253 & 0,0881 & 0,5253 & 0,0881 \\
\hline 647 & 79 & 0,4788 & 0,0893 & 0,4788 & 0,0893 & 0,4788 & 0,0893 & 0,4788 & 0,0893 \\
\hline 739 & 80 & 0,4028 & 0,0580 & 0,4028 & 0,0580 & 0,4028 & 0,0580 & 0,4028 & 0,0580 \\
\hline
\end{tabular}




\section{CONCLUSÕES}

A metodologia proposta para a modelagem de dados provenientes de bioensaios de toxicidade com isolados de B. bassiana, conforme descrito na Seção 3 , como alternativa às abordagens usuais através da utilização do modelo multinomial acumulado e dos modelos de superdispersão Dirichlet-multinomial, REMI e REMII, apresentou resultados satisfatórios na estimação dos parâmetros do modelo da regressão ( $\hat{\boldsymbol{\beta}}^{\prime}$ 's) e na estimação do tempo letal $50\left(L T_{50}\right)$ e do tempo letal 90 $\left(L T_{90}\right)$.

Conforme demonstrado na Seção 4, as etimativas dos parâmetros da regressão não são afetadas pelas diferentes escolhas da função de variância (20), (27) ou (35) associada a cada modelo. Assim, como consequência, as estimativas para $L T_{50}$ e $L T_{90}$ vão ser sempre as mesmas, pois são funções apenas dos $\hat{\boldsymbol{\beta}}$ 's. De forma análoga ao que ocorre com o modelo logístico para dados de dose-resposta com distribuição binomial, o modelo logístico para dados com distribuição multinomial apresentado neste trabalho, mostrou resultados semelhantes, isto é, a estimação dos $\beta$ 's, $L T_{50}$ e $L T_{90}$ é robusta à escolha da estrutura da função de variância do modelo.

A metodologia proposta foi implementada no pacote computacional SPLUS (MathSoft, Inc. Seattle, Washington, USA) na versão 3.4, permitindo dessa forma, o uso das diferentes parametrizações na modelagem da superdispersão.

O uso da distribuição Dirichlet-multinomial parece inflacionar os erros padrões associados aos $\hat{\boldsymbol{\beta}}$ 's, quando comparado com os demais modelos. 
Os modelos de efeitos aleatórios no preditor linear (REMI e REMII) apresentaram-se como uma alternativa viável para a modelagem da superdispersão, visto que existe um comportamento de variabilidade natural entre as réplicas (grupos) dentro de cada isolado. Essa variabilidade é observada de forma um pouco mais acentuada nos resultados do bioensaio com os cupins.

Apesar de o número de replicas por isolado ter sido diferente em cada bioensaio ( $K=5$ no bioensaio com os cupins e $K=12$ no bioensaio com os ácaros), os pesquisadores afirmam que bioensaios com cupins sempre produzem resultados com uma maior variabilidade nos dados do que bioensaios que utilizam ácaros. Essa é uma característica de comportamento natural inerente aos cupins, segundo os entomologistas.

O uso do grupo (cluster) como unidade experimental, aliado ao fato do comportamento de variabilidade natural associada a cada artrópodo (cupim e ácaro), ressalta, ainda mais, a necessidade de pesquisa e uso de modelos estatísticos mais complexos que possam levar em consideração essa variabilidade inerente aos dados que, de forma genérica, não é levada em consideração na variância nominal dos modelos usualmente utilizados.

Apesar de não ter ocorrido problemas na estimação dos parâmetros do modelo da regressão ( $\hat{\boldsymbol{\beta}}$ 's), a estrutura particular do preditor linear, no qual foi considerado apenas o tempo como variável regressora, parece levar ao resultado de

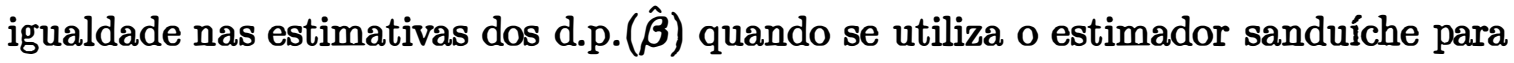
a $\operatorname{Var}(\hat{\boldsymbol{\beta}})$, dado por (46). Esses resultados sugerem que o uso desse estimador seja melhor estudado, para esse preditor linear em particular, ou então o mesmo não seja utilizado para as inferências do modelo, optando-se assim pela matriz de variâncias e covariâncias assintótica dada por (45).

No experimento com os cupins, foram consideradas cinco repetições $(K=5)$ para cada isolado, com trinta insetos por grupo $(n=30)$, sendo as observações feitas em oitos tempos $(D=8)$. Nesse caso, talvez fosse mais indicado, para futuros experimentos, usar um número maior de repetições por réplica $(K \geq D)$. 
Para o experimento com os ácaros, o número de repetições por isolado $(K=12) \mathrm{e}$ o número de ácaros por repetição $(n=10)$ está razoável, dado que as observações foram feitas em seis tempos $(D=6)$. A consideração a ser feita para esse bioensaio é que seja redurido o número de folhas de feijão utilizadas em cada placa de Petri, por exemplo de doze para seis folhas, com duas folhas de feijão em cada. Desse modo, as placa poderiam ser consideradas como as repetições $(K=6)$ e, para cada repetição, ter-se-iam vinte ácaros $(n=20)$. Isso poderia levar a uma melhoria na qualidade das estimativas dos $\beta$ 's, visto que para os modelos de efeito aleatório REMI e REMII a variabilidade entre os interceptos e entre os coeficientes angulares das retas de regressão está diretamente relacionada com os parâmetros de superdispersão considerados em cada modelo, $\sigma^{2}$ e $\left(\nu^{2}, \tau^{2}\right)$ respectivamente.

Considerando-se que, independente do modelo adotado, as estimativas dos parâmetros da regressão, $\hat{\boldsymbol{\beta}}$ 's, vão ser os mesmos e, como consequência, as estimativas de $L T_{50}$ e $L T_{90}$ também, os pesquisadores, em uma triagem inicial, já podem descartar os patógenos que apresentaram resultados nos quais $L T_{50}>8$ dias (para o bioensaio com os cupins) e $L T_{50}>6$ dias (para o bioensaio com os ácaros). Desse modo, os experimentos no campo já podem ser iniciados com os patógenos que apresentaram um grau de virulência considerado aceitável para o intervalo de tempo de realização dos experimentos. 


\section{REFERÊNCIAS BIBLIOGRAFICAS}

AGRESTI, A. Categorical data analysis. New York: John Wiley and Sons, 1990. 558p.

AGRESTI, A. An introduction to categorical data analysis. New York: John Wiley and Sons, 1996. 290p.

AITKIN, M.; ANDERSON, D.A.; FRANCIS, B.; HINDE, J.P. Statistical modelling in GLIM. Oxford: Oxford University Press, 1989.

ALBERT, P.S. Tutorial in Biostatistics Longitudinal Data Analysis (Repeated Measures) in Clinical Trials. Statistics in Medicine, 18, p.1707-1732, 1999.

ALMEIDA, J.E.M. Avaliação de fungos entomopatogénicos visando ao controle de cupim subterrâneo Heterotermes tenuis (Hagen, 1858) (Isoptera; Rhinotermitidae). Piracicaba, 1994. 105p. Dissertação (Mestrado) - Escola Superior de Agricultura "Luiz de Queiroz", Universidade de São Paulo.

ALMEIDA, J.E.M.; ALVES, S.B.; PEREIRA, R.M. Selection of Beauveria spp. isolates for control of the termite Heterotermes tenuis (Hagen, 1958). Jour. Appl. Ent. 121, p.539-543, 1997.

ALVES, S.B. Controle Microbiano de Insetos. 2.ed. Piracicaba: FEALQ, 1998. $1163 p$.

ARTES, R. Extensões da Teoria das Equações de Estimação Generalizadas a Dados Circulares e Modelos de Dispersão. São Paulo, 1997. 130p. Tese (Doutorado) 
- Instituto de Matemática e Estatística, Universidade de São Paulo.

BETENSKY, R.A.; WILLIAMS, P.L. A comparison of models for clustered binary outcomes: analysis of a designed immunology experiment. Applied Statistics, 50, p.43-61, 2001.

BICKEL, P.J.; DOKSUM, K.A. Mathematical Statistics. Oakland: Holden-day, Inc., 1977. 492p.

BOX, G.E.P.; COX, D.R. An Analysis of transformations. Journal of the Royal Statistical Society Serie B, 26, p.211-263, 1964.

BRESLOW, N.E. Extra-Poisson variation in log-linear models. Applied Statistics, 33, p.38-44, 1984.

BRESLOW, N.E.; CLAYTON, D.G. Approximate inference in generalized linear models. Journal of American Statistical Association, 88, p.9-25, 1993.

CARTER, E.M.; HUBERT, J.J. A growth-curve model approach to multivariate quantal bioassay. Biometrics, 40, p.699-706, 1984.

COCHRAN, W.G. Analysis of variance for percentages based on unequal numbers. Journal of the American Statistical Association, 38, p.287-301, 1943.

COLLET, D. Modelling binary data. London: Chapman \& Hall, 1991. 369p.

CORDEIRO, G.M. Modelos Lineares Generalizados. Campinas: VII SINAPE, 1986. 286p.

COX, D.R. Regression models and life tables. Journal of the Royal Statistical Society Serie B, 34, p.187-220, 1972.

DEMÉTRIO, C.G.B. Modelos Lineares Generalizados na Experimentaçāo Agronômica, $5^{\circ}$ SEAGRO e $38^{a}$ RBRAS. Porto Alegre, DE/UFRGS, 1993. 
$125 \mathrm{p}$.

DEMÉTRIO, C.G.B. A Superdispersão em Dados de Produção de Brotação da Cana-de-Açúcar e de Proporção de Perfilhos com Carvão. Piracicaba, 1994. 165p. Tese (Livre-Docência) - Escola Superior de Agricultura "Luiz de Queiroz", Universidade de São Paulo.

DEMÉTRIO, C.G.B.; HINDE, J.P. Half-Normal plots and overdispersion. GLIM Newsletter, 27, p.19-26, 1997.

DOBSON, A.J. An introduction to generalized linear models. 2.ed. London: Chapman \& Hall, 1990. 174p.

FERNANDES, P.M.; ALVES, S.B. Controle de Cornitermes cumulans (Kolar, 1832)(Isoptera; Termitidae) utilizando Beauveria bassiana (Bals.) Vuill. e Metarhizium anisopliae (Metsch.) Sorok. em condições de campo. Anais da Sociedade de Entomologia do Brasil, 20, p.45-49, 1991.

FINNEY, D.J. Probit Analysis. Cambridge: Cambridge Univ. Press, 1971.

FINNEY, D.J. The estimation from individual records of the relationship between dose and quantal response. Biometrika, 34, p.320-334, 1947.

FREEMAN, D.H. Applied Categorical Data Analysis. Statistics: textbooks and monographs, v.79. New York: Marcel Dekker, 1987. 318p.

GLONEK, G.F.V. A class of regression models for multivariate categorical responses. Biometrika, 83, p.15-28, 1996.

GRENN, P.J. Iteratively reweighted least squares for maximum likelihood estimation, and some robust and resistant alternatives (with Discussion). Journal of the Royal Statistical Society, Serie B, 46, p.149-192, 1984. 
GRIZZLE, J.E.; STARMER, C.F.; KOCH, G.G. Analysis of categorical data by linear models. Biometrics, 25, p.489-504, 1969.

HARTLEY, H.O.; SIELKEN, R.L.Jr. Estimation of "safe doses" in carcinogenic experiments. Biometrics, 33, p.1-30, 1977.

HALL, D.B. Estimating equations for clustered data based on extended quasilikelihood. In: INTERNATIONAL BIOMETRIC CONFERENCE, Cape Town, 1998. Proceedings. 1998. p.45-54.

HEWLETT, P.S. Time from dosage death in beetles Tribolium castaneum, treated with pyrethrins or DDT, and its bearing on dose-mortality relations. Journal of Product Research, 10, p. 27-41, 1974.

HINDE, J.P. Compound Poisson regression models. In GLIM 82. ed. R. Gilchrist, New York: Springer, p.109-121, 1982.

HINDE, J.P. Macros for fitting overdispersion models. GLIM Newsletter, 26, p.10-19, 1996.

HINDE, J.P.; DEMÉTRIO, C.G.B. Modelling with Overdispersion. In: $11^{\text {th }}$ INTERNATIONAL WORKSHOP ON STATISTICAL MODELLING, Orvieto, 1996. Proceedings. Orvieto: Statistical Modelling, 1996. p.200-201.

HINDE, J.P.; DEMÉTRIO, C.G.B. Overdispersion: models and estimation. Computational Statistics and Data Analysis, 27, p.151-170, 1998a.

HINDE, J.P.; DEMÉTRIO, C.G.B. Overdispersion: models and estimation. A Short Course for SINAPE 1998. Caxambu: XIII SINAPE, 1998b. 73p. HUSSEY; HUFFAKER. 1976.

LAWLESS, J.F. Statistical models and methods for lifetime data. New York: Wiley, 1982. 
LIANG, K.-Y.; ZEGER, S.L. Longitudinal data analysis using generalized linear models. Biometrika, 73, p.13-22, 1986.

LINDSEY, J.K. Models for repeated measurements. Clarendon Press-Oxford, 1993.

McCULLAGH, P. Regression models for ordinal data (with Discussion). Journal of the Royal Statistics Society Serie B, 42, p.109-151, 1980.

McCULLAGH, P.; NELDER, J.A. Generalized Linear Models. 2.ed. London: Chapman \& Hall/CRC, 1989. 511p.

MOORE, D.F. Modelling the Extraneous Variance in the Presence of Extrabinomial Variation. Applied Statistics, 36, p.8-14, 1987.

MORGAN, B.J.T. Analysis of Quantal Response Data. Londres: Chapman \& Hall, 1992. 511p.

MORGAN, B.J.T. Statistics in Toxicology. Clarendon Press-Oxford, 1996. 224p.

MOSIMANN, J.E. On the compound multinomial distribution, the multivariate $\beta$-distribution, and correlations among proportions. Biometrika, 49, p.65, 1962.

NELDER, J.A.; WEDDERBURN, R.W.M. Generalized linear models. Journal of Royal Statistical Society Serie A, 135, p.370-384, 1972.

NOVARETTI, W.R.T. Controle de cupins em cana-de-açúcar através do emprego de inseticidas de solo. Boletim Técnico Copersucar, 38, São Paulo, 1985.

OGLIARI, P.J. Piracicaba, 1999. 156p. Tese (Doutorado) - Escola Superior de Agricultura "Luiz de Queiroz", Universidade de São Paulo. 
O'HARA HINES, R.J.; LAWLESS, J.F. Modelling Overdispersion in Toxicological Mortality Data Grouped over Time. Biometrics, 49, p.107-121, 1993.

O'HARA HINES, R.J.; LAWLESS, J.F.; CARTER, E.M. Diagnostics for Cumulative Multinomial Generalized Linear Model, with applications to Grouped Toxicological Mortality Data. Australian Journal of Statistics, 87, n.420, p.1059-1069, 1992.

PAUL, S.R.; LIANG, K.Y.; SELF, S.G. On Testing Departure from the Binomial and Multinomial Assumptions. Biometrics, 45, p.231-236, 1989.

PETKAU, A.J.; SITTER, R.R. Models for Quantal Response Experiments over Time. Biometrics, 45, p.1299-1308, 1989.

PIERCE, D.A.; STEWART, W.H.; KOPECKY, K.J. Distribution-free regression analysis of grouped survival data. Biometrics, 35, p.785-793, 1979.

PRENTICE, R.L.; GLOECKLER, L.A. Regression analysis of grouped survival data with application to breast cancer data. Biometrics, 34, p.57-67, 1978.

PURI, P.S. A linear birth and death process under the influence of another process. Journal Applied Probability, 12, 1, p.1-17, 1975.

RAO, C. R. Linear Statistical Inference and Its Applications. New York: John Wiley and Sons, 1973. 625p.

RIDOUT, M.S., FENLON, J.S.; HUGHES, P.R. A generalised one hit-model for bioassays of insect viruses. Biometrics, 49, p.1136-1141, 1993.

SPYRIDES-CUNHA, M.H. Modelos para dados categorizados ordinais: aplicações em agropecuária. Piracicaba, 1998. 80p. Dissertação (Mestrado) - Escola Superior de Agricultura "Luiz de Queiroz", Universidade de São Paulo. 
STIRATELLI, R., LAIRD, N.M.; WARE, J.H. Random-effects models for serial observations with binary response. Biometrics, 40, p.961-971, 1984.

TAMAI, M.A. Avaliação de funfos entomopatogênicos para o controle de Tetranychus urticae Koch. Piracicaba, 1997. 85p. Dissertação (Mestrado) - Escola Superior de Agricultura "Luiz de Queiroz", Universidade de São Paulo.

TERRIL, P.K.; MORGAN, J.T.; FENLON, J.S. Statistical Models for Biological Control. In: The XIXth International Biometric Conference, Cape Town, South Africa, 1998. Resumos.

VIEIRA; A.M.C. Modelos para dados de proporções com superdispersão aplicados ao controle biológico. Piracicaba, 1998. 61p. Dissertação (Mestrado) - Escola Superior de Agricultura "Luiz de Queiroz", Universidade de São Paulo.

VONESH, E.F.; CHINCHILLI, V.M. Linear and Nonlinear Models for the Analysis of Repeated Measurements. New York: Marcel Dekker, Inc., 1997. 560p.

WEDDERBURN, R.W.M. Quasi-likelihood functions, generalized linear models, and the Gauss-Newton method. Biometrika, 61, 3, p.439-447, 1974.

WHITEHEAD, J. The analysis of colapse clinical trials, with application to comparison of two ulcer treatments. Statistics in Medicine, 8, p.1439-1454, 1989.

WILLIAMS, D.A. Extra-binomial variation in logistic-linear models. Applied Statistics, 31, p.144-148, 1982.

WOLFE, R. General purpose macros to fit models to an ordinal response. GLIM Newslwtter, 26, p.20-27, 1996.

ZEGER, S.L., LIANG, K.-Y. Longitudinal data analysis for discrete and continuous outcomes. Biometrics, 42, p.1049-1060, 1986. 
ZEGER, S.L., LIANG, K.-Y.; ALBERT, P.S. Models for longitudinal data: A generalized estimating equation approach. Biometrics, 44, p.1049-1060, 1988. 


\section{APENNDICE 1}

\section{Programas computacionais feitos no SPLUS}




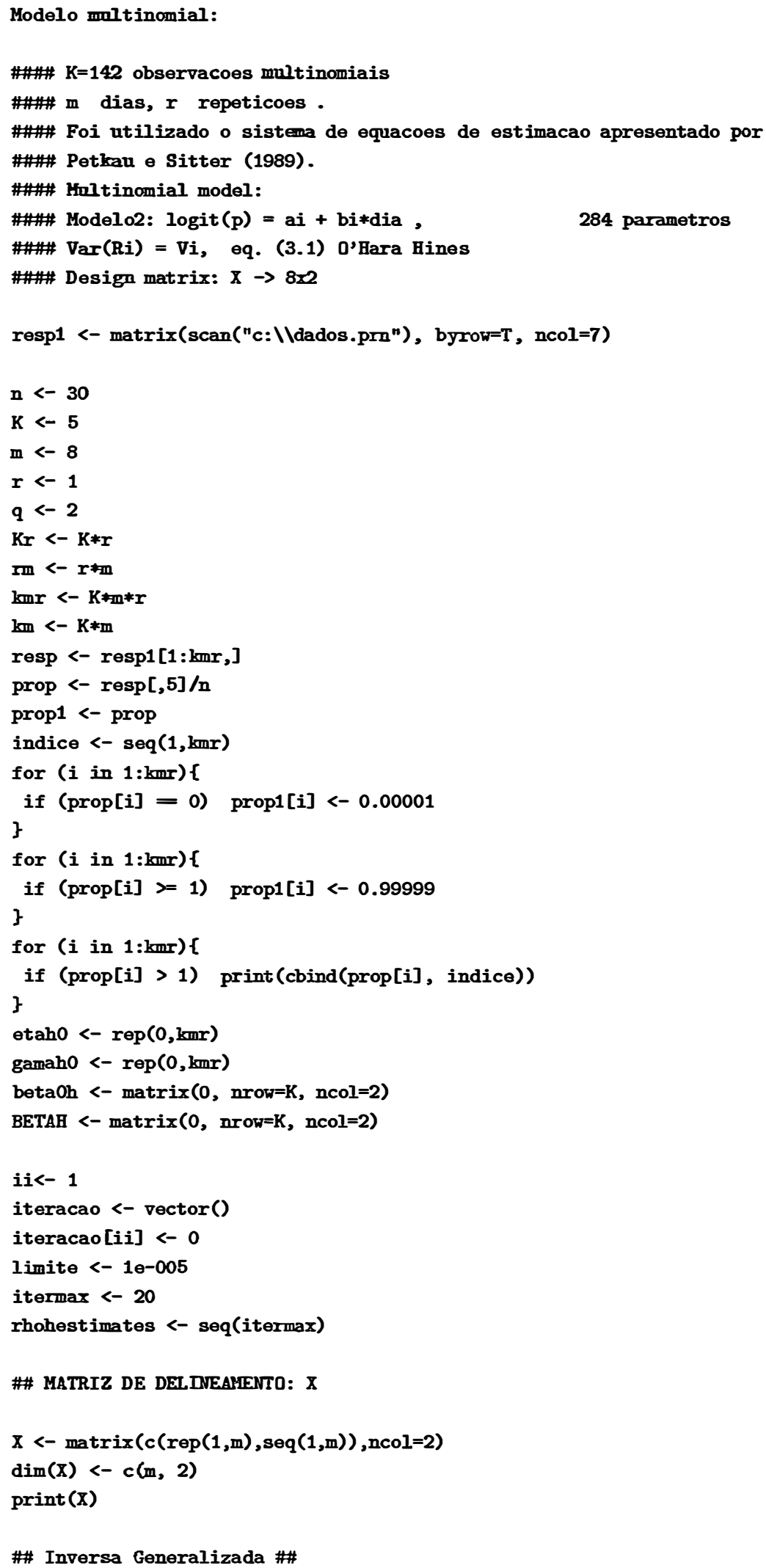




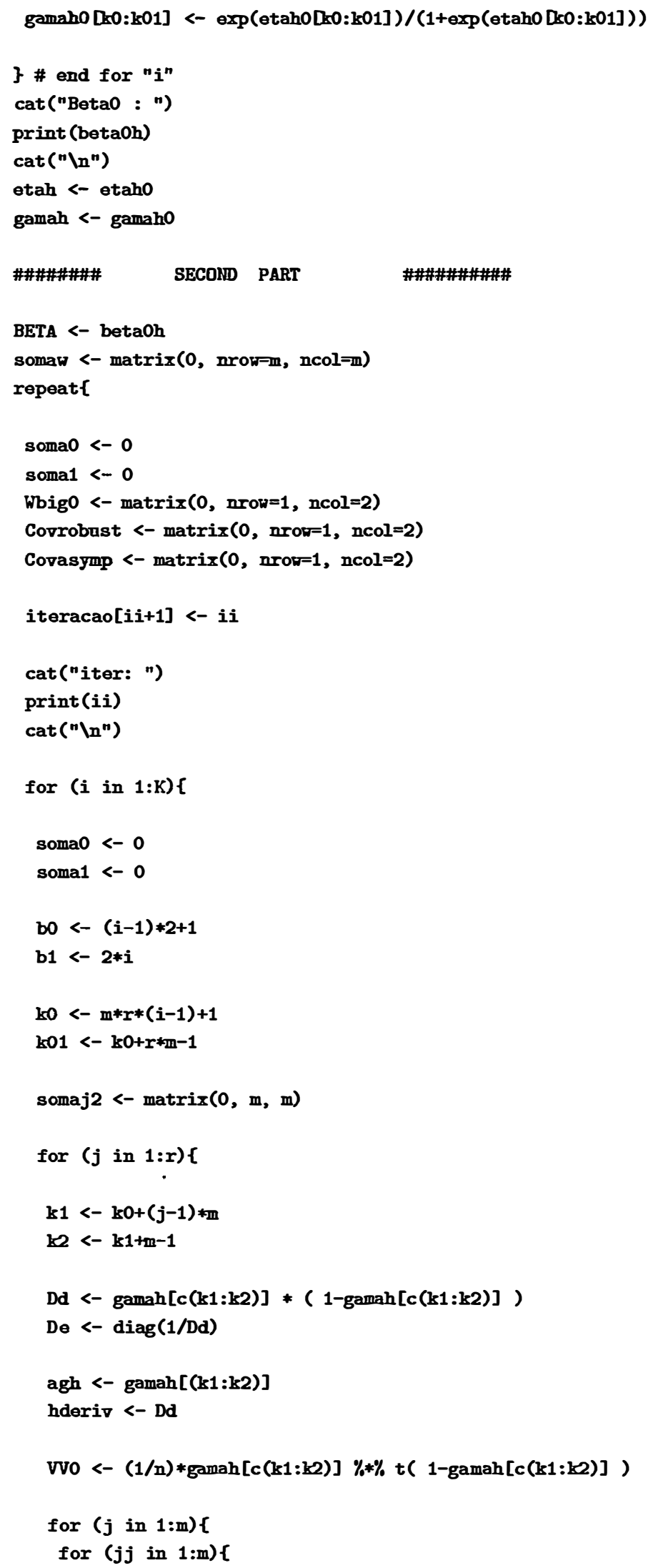




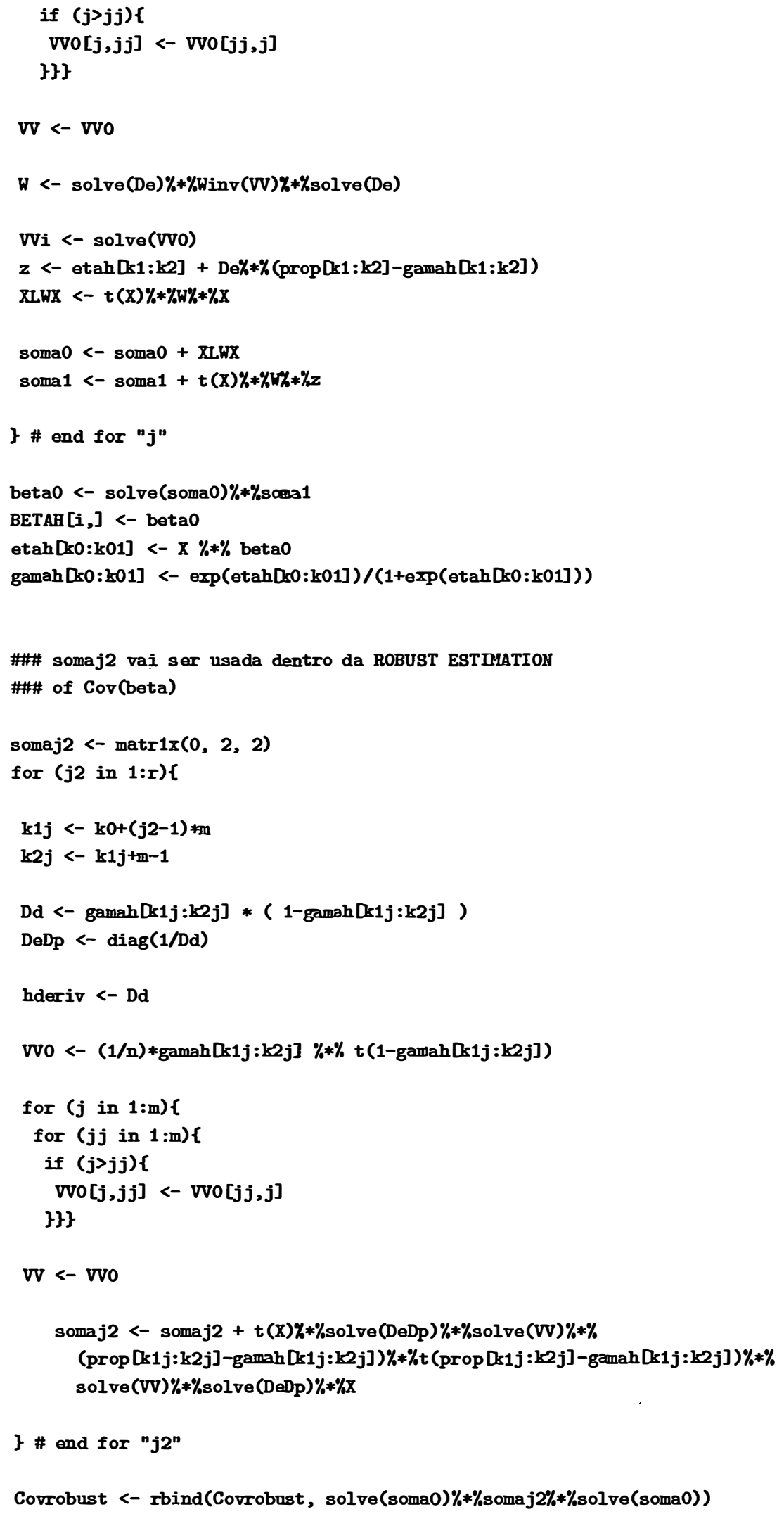




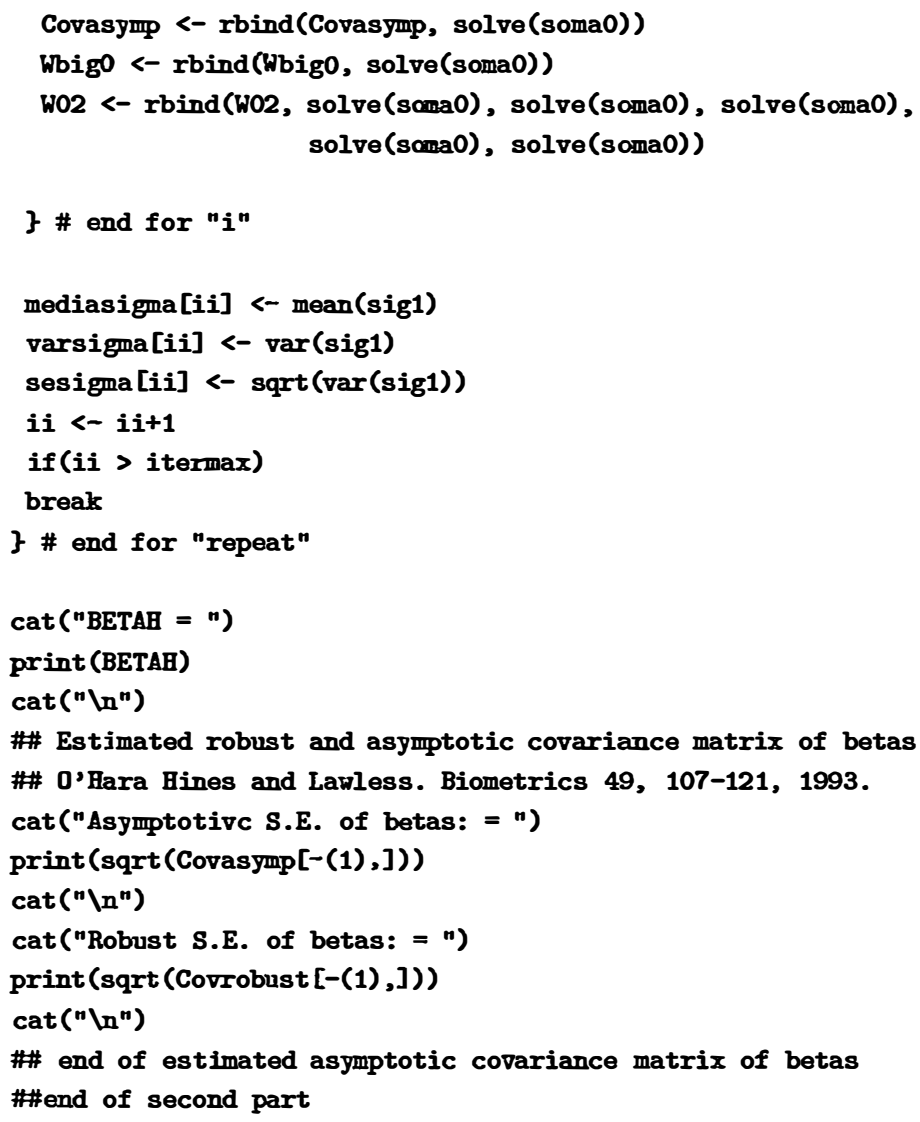

Modelo REMI:

\#\# TERMITE data set. 1 modelo E UM SIGMA2 para cada isolado. 


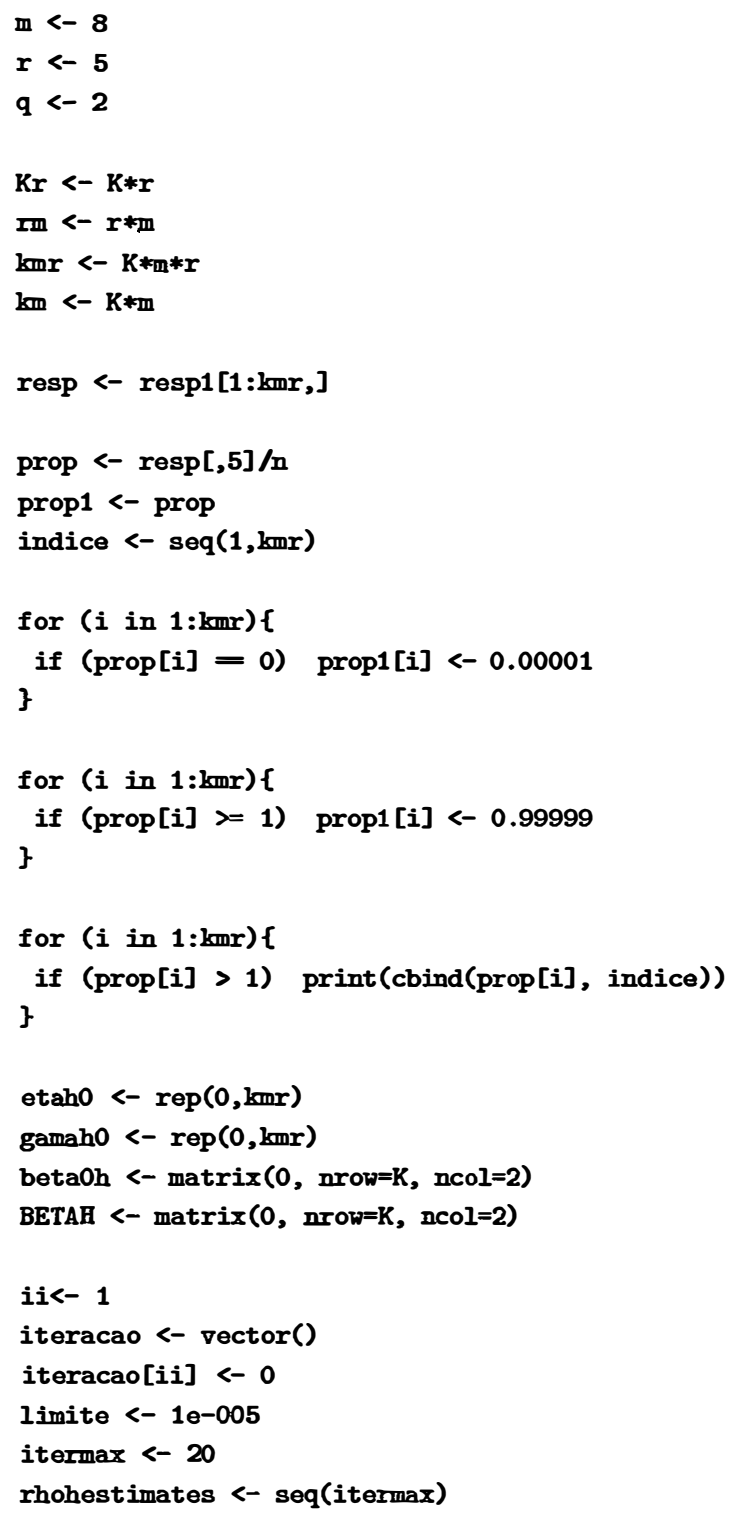


$\mathrm{nz}<-s \$ d>$ tol * s\$d[1]

if $(\operatorname{ang}(n z)) s \$ v[, n z] \% * \%(t(s \$ u[, n z]) / s \$ d[n z])$ else $A * 0$ \}

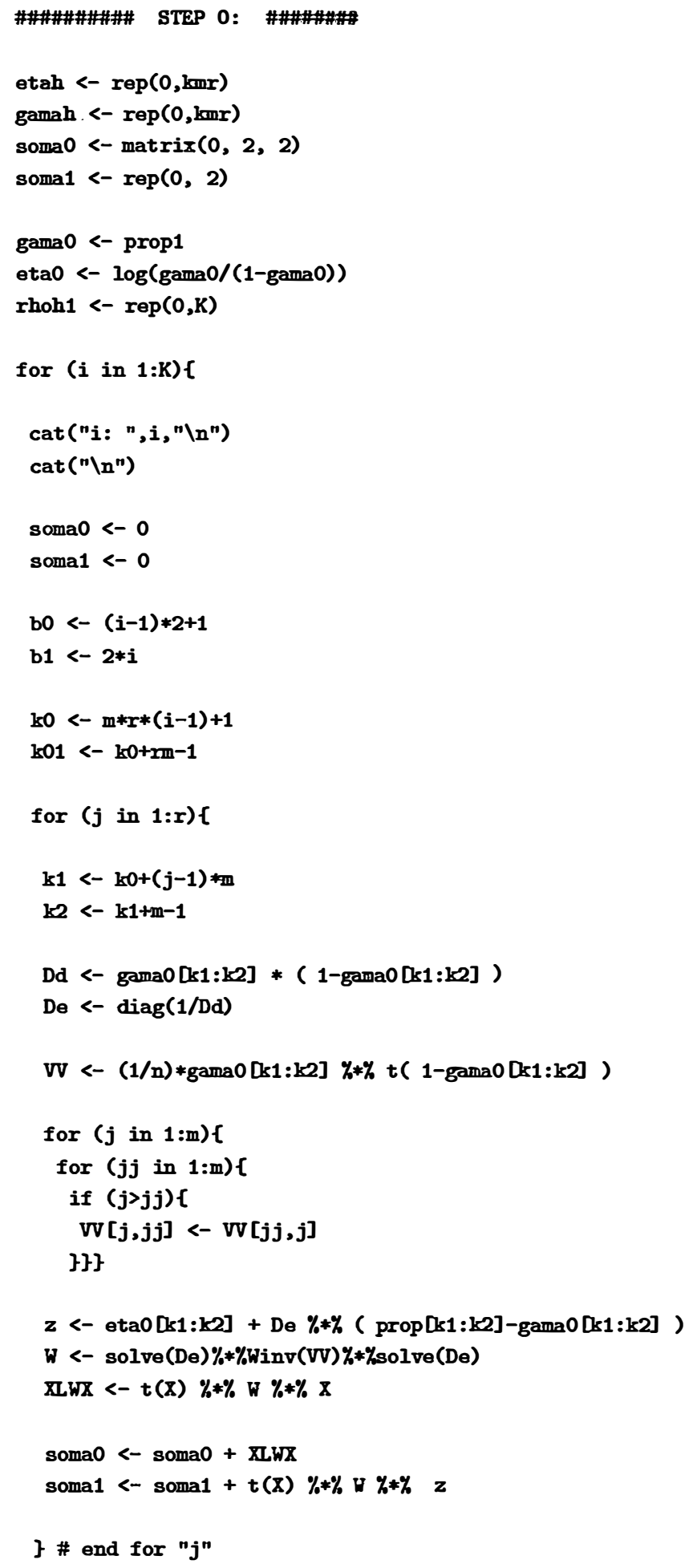




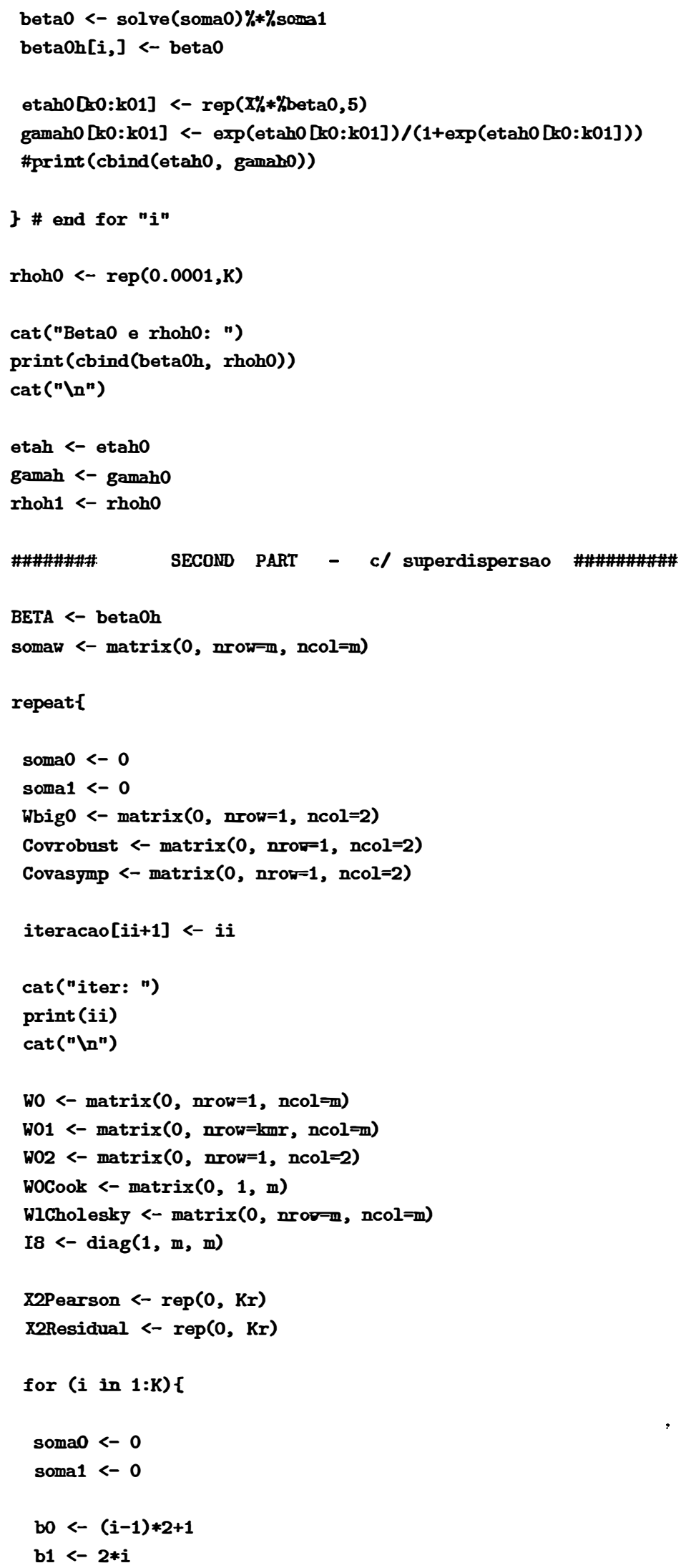




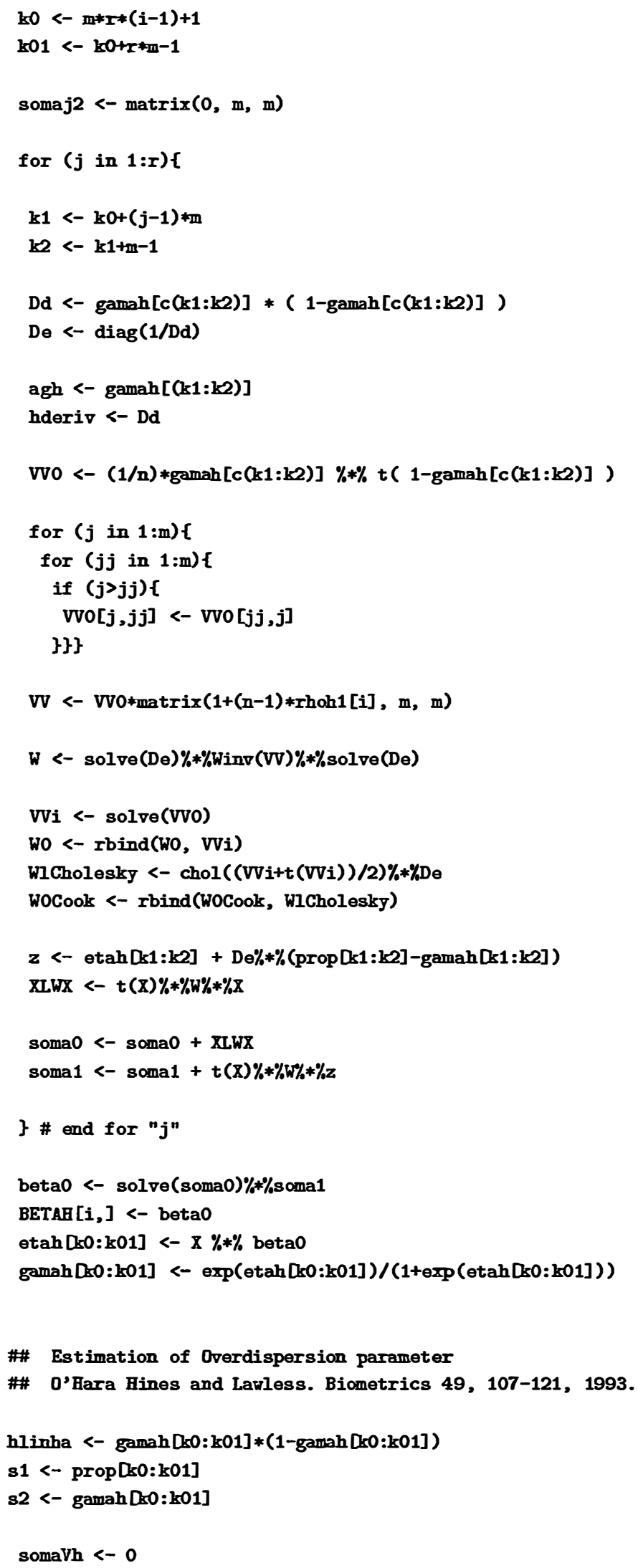




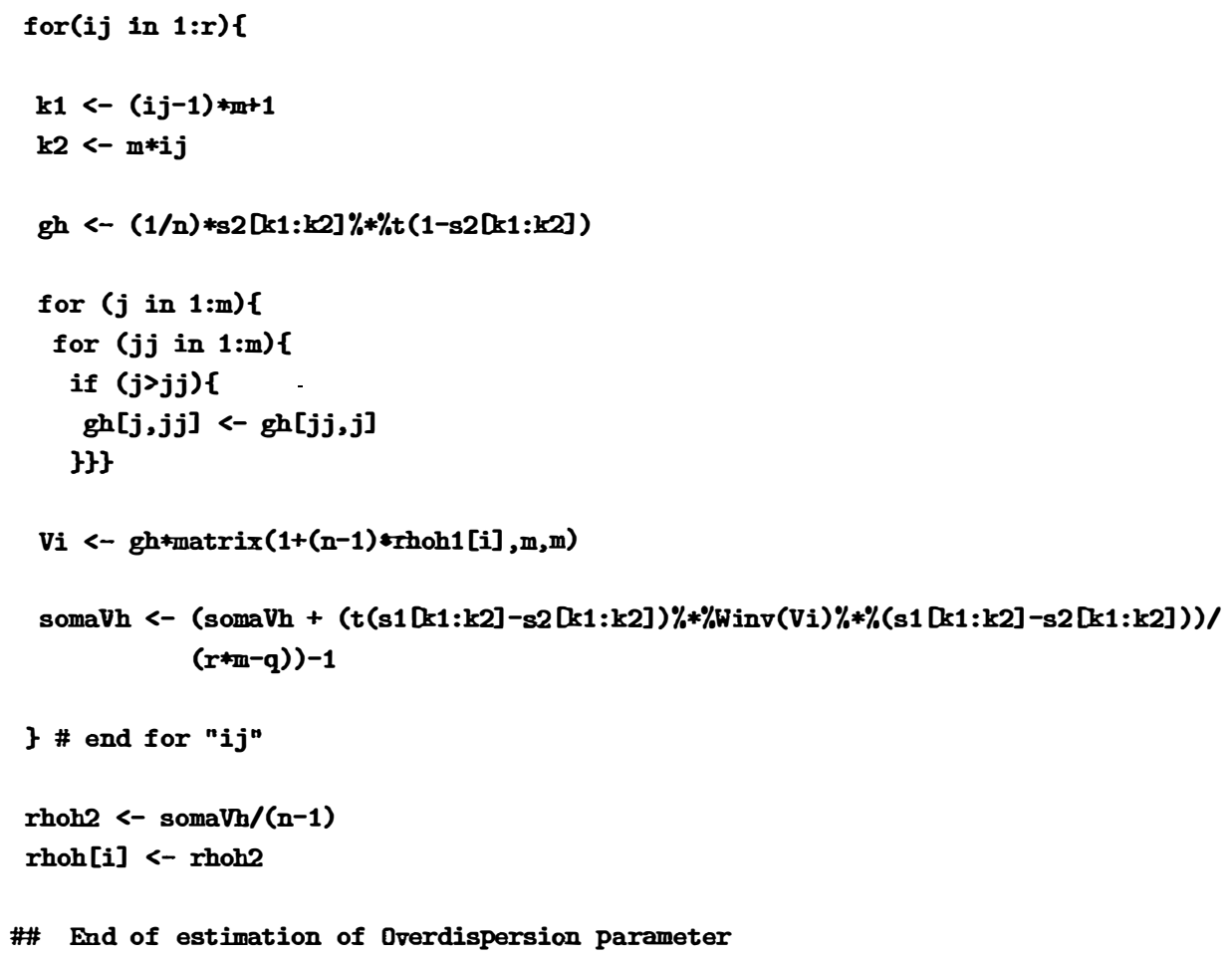




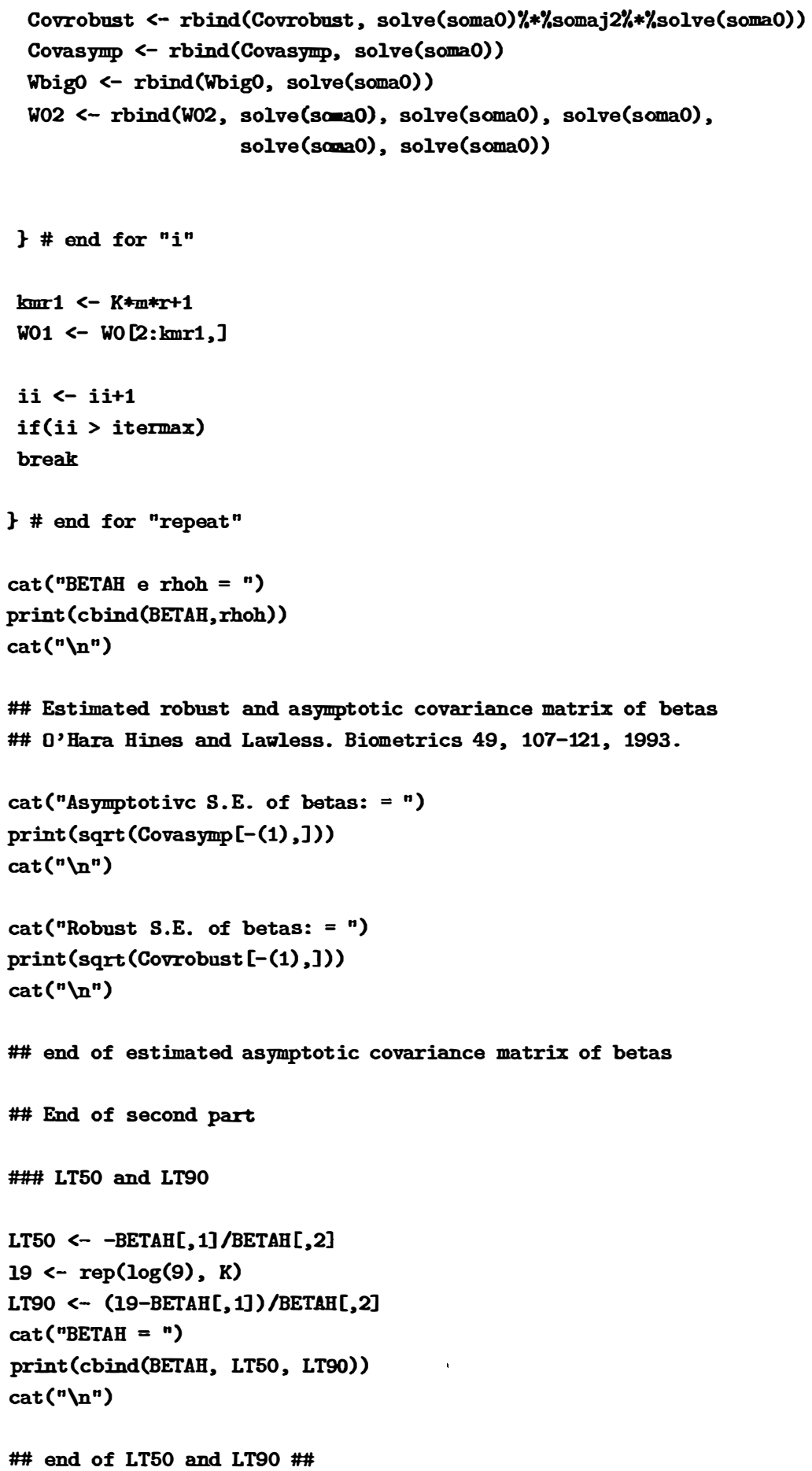


Modelo REHT :

K=142 observacoes multinomiais

\#作 dias, r=5 repeticoes.

\#\# Foi utilizado o sistema de equacoes de estimacao apresentado por \#\# Petkan e Sitter (1989).

\#\#andom effect model:

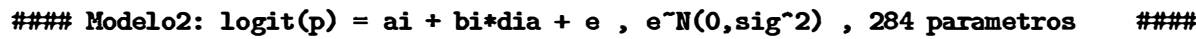

\#\#\#

\#ar $(\mathrm{Ri})=\mathrm{Vi}+(1-1 / \mathrm{n}) *\left(\mathrm{~s}^{\wedge} 2\right) * \mathrm{~h}^{\prime}($ etai)*t(h'(etai)),

\#\# eq. (3.4b) O'Hara Hines

\#\#

\#\#\#

\#\#\#

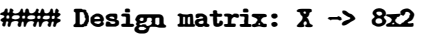

\#\#\#

\#\#\#

resp1 <- matrix(scan("c: |lsergiollcupiml|Sergio41.prn"), byrow=T, ncol=7)

n $<-30$

K $<-10$

m $<-8$

$r<-5$

q $<-2$

$\mathrm{Kr}<-\mathrm{K} * \mathrm{r}$

Im $<-r * m$

$\mathrm{kmr}<-\mathrm{K} * \mathrm{~m} * \mathrm{r}$

$\mathrm{km}<-\mathrm{K} * \mathrm{~m}$

resp <- resp1 $[1: \mathrm{kmr}$,

prop <- resp $[, 5] / n$

prop1 <- prop

indice <- seq $(1, \mathrm{kmr})$

for ( $i$ in $1: \mathrm{kmr}$ ) [

if (prop $[i]=0) \quad \operatorname{prop1}[i]<-0.00001\}$

for ( $i$ in $1: k m r)$ r

if (prop[i] > = 1) prop1[i] <- 0.99999\}

for ( $i$ in $1: \mathrm{kmr}$ ) ?

if (prop[i] > 1) print(cbind(prop[i], indice)) \}

etaho <- $\operatorname{rep}(0, \mathrm{kmr})$

gamaho <- rep $(0, \mathrm{kmr})$

beta0h <- matrix (0, nrow=K, ncol=2)

BETAH <- matrix (0, nrow=K, ncol=2)

ii<- 1

iteracao <- vector ()

iteracao[ii] <- 0

limite <- 1e-005

itermax $<-20$

rhohestimates <- seq(itermax) 
\#\# MATRIZ DE DEIINEAYENTO: $X$

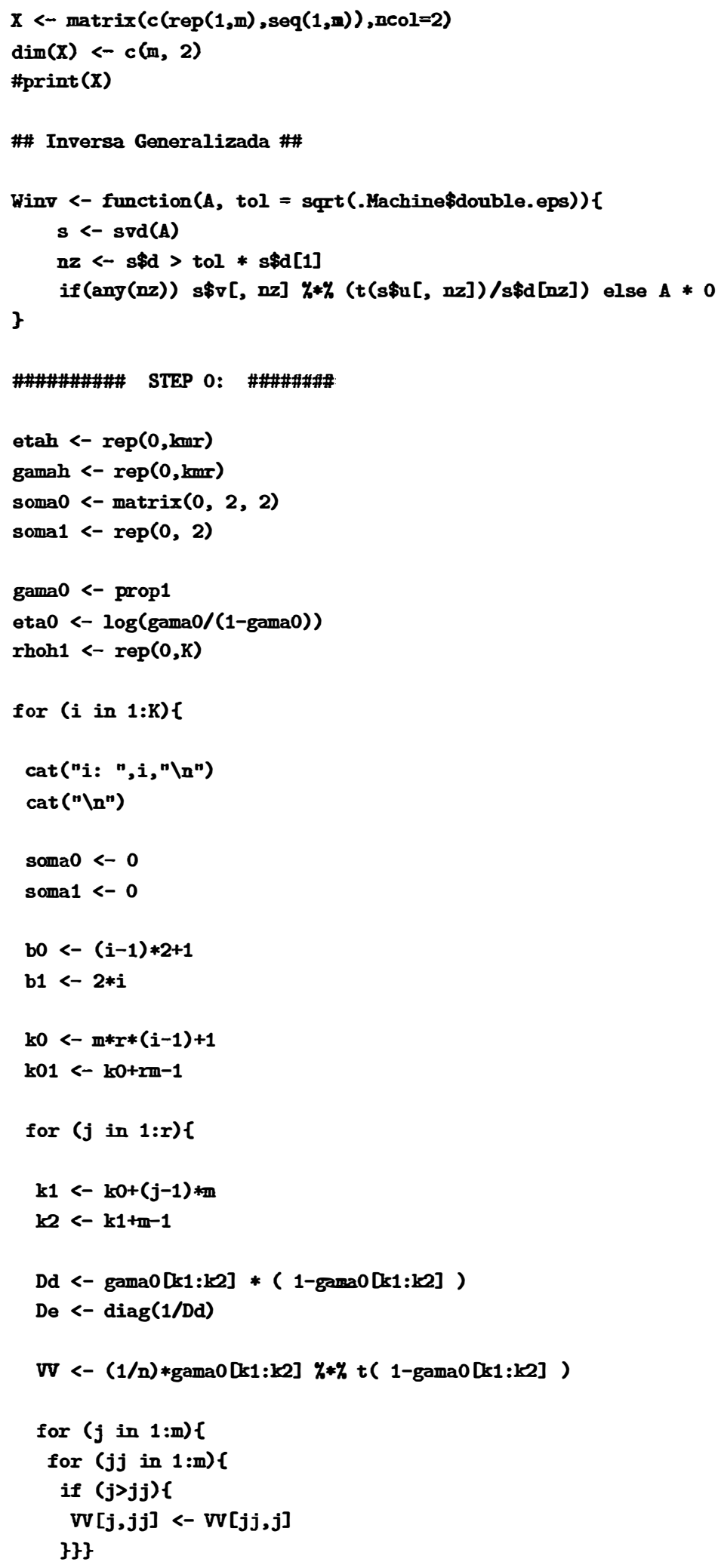




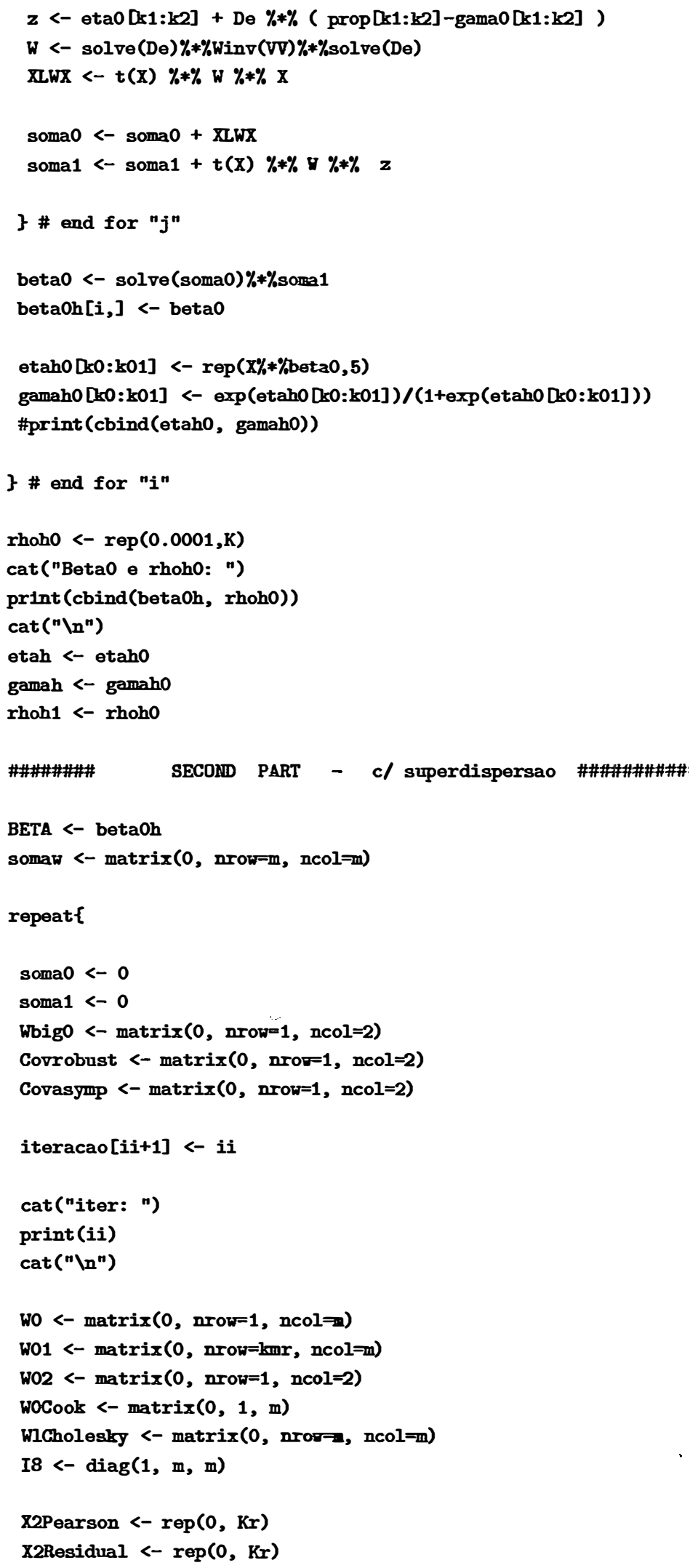




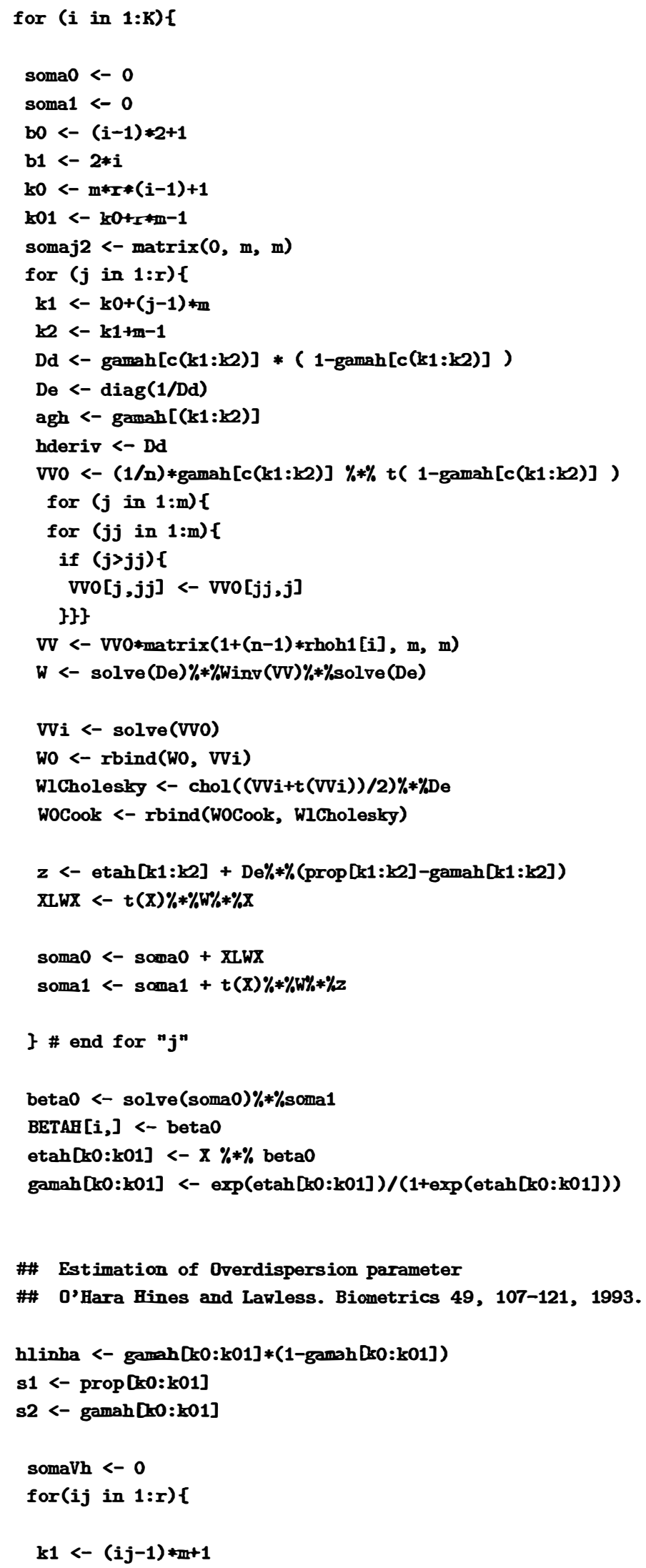




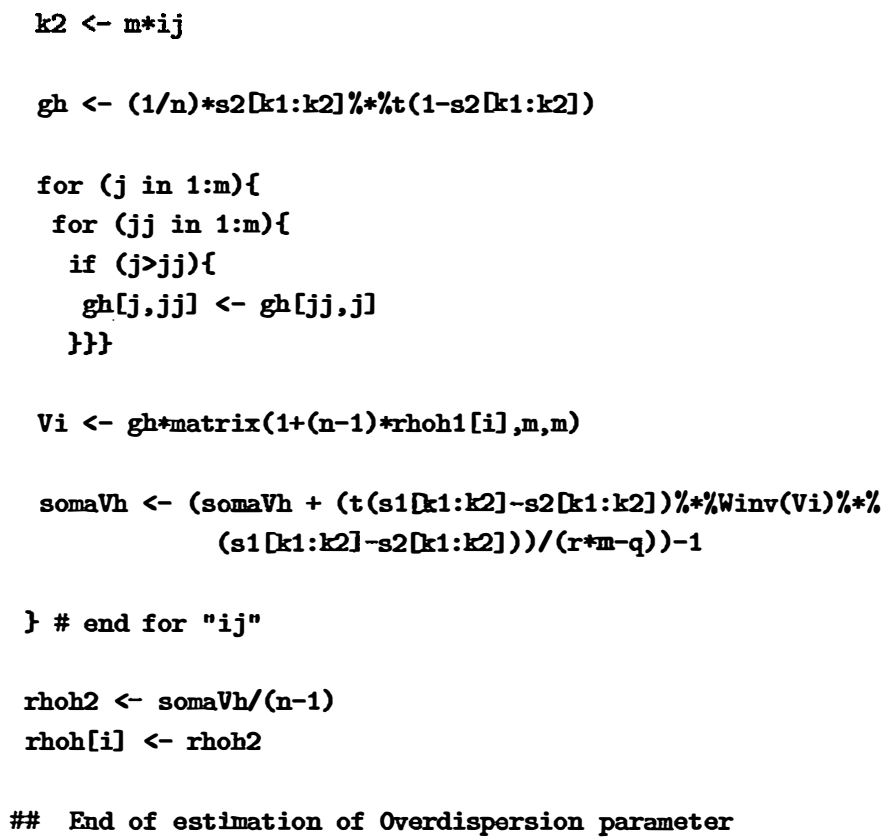




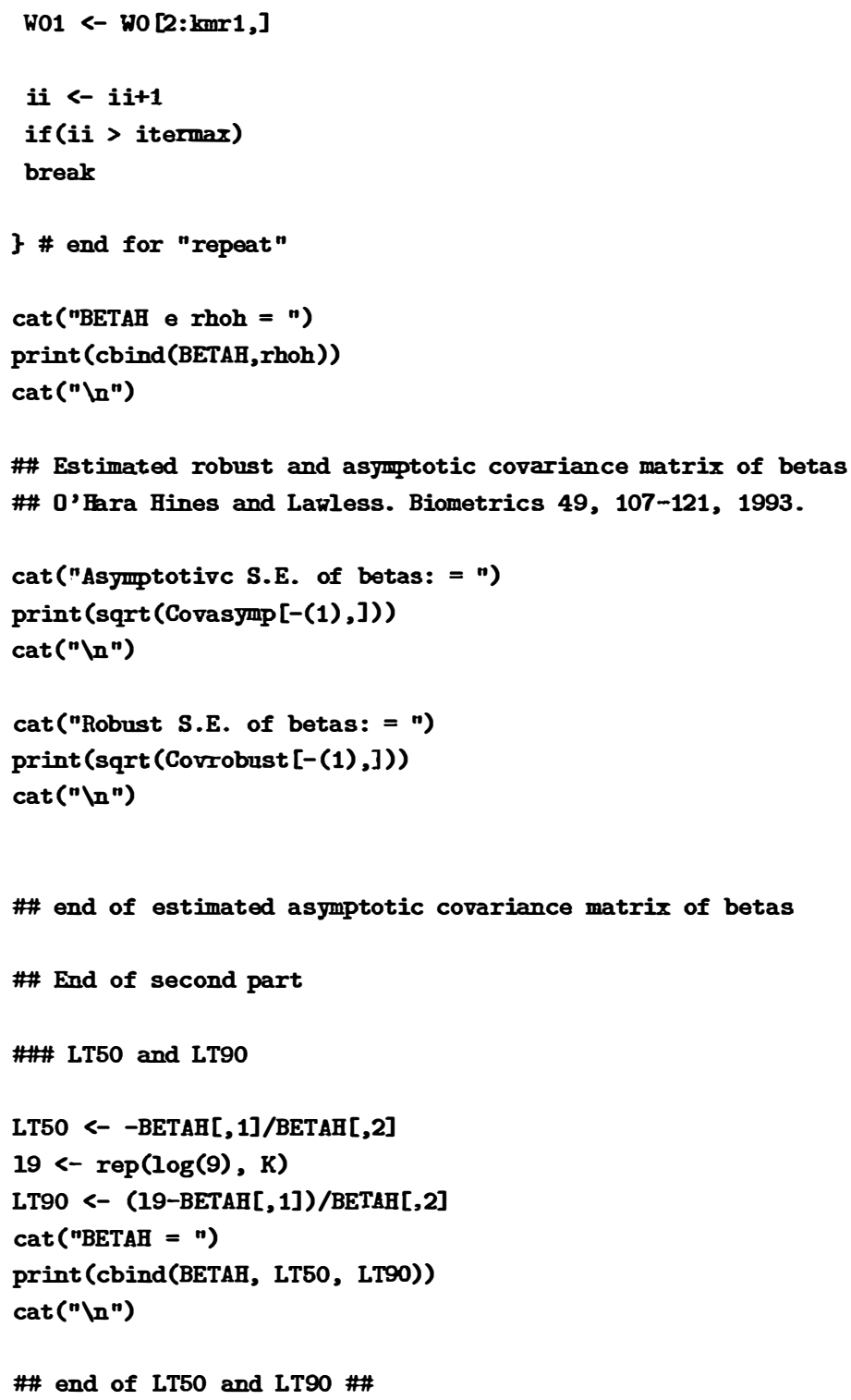

Modelo REAIII :

\#\# TERMITE data set. 1 modelo E UM SIGMA2 para cada isolado. 


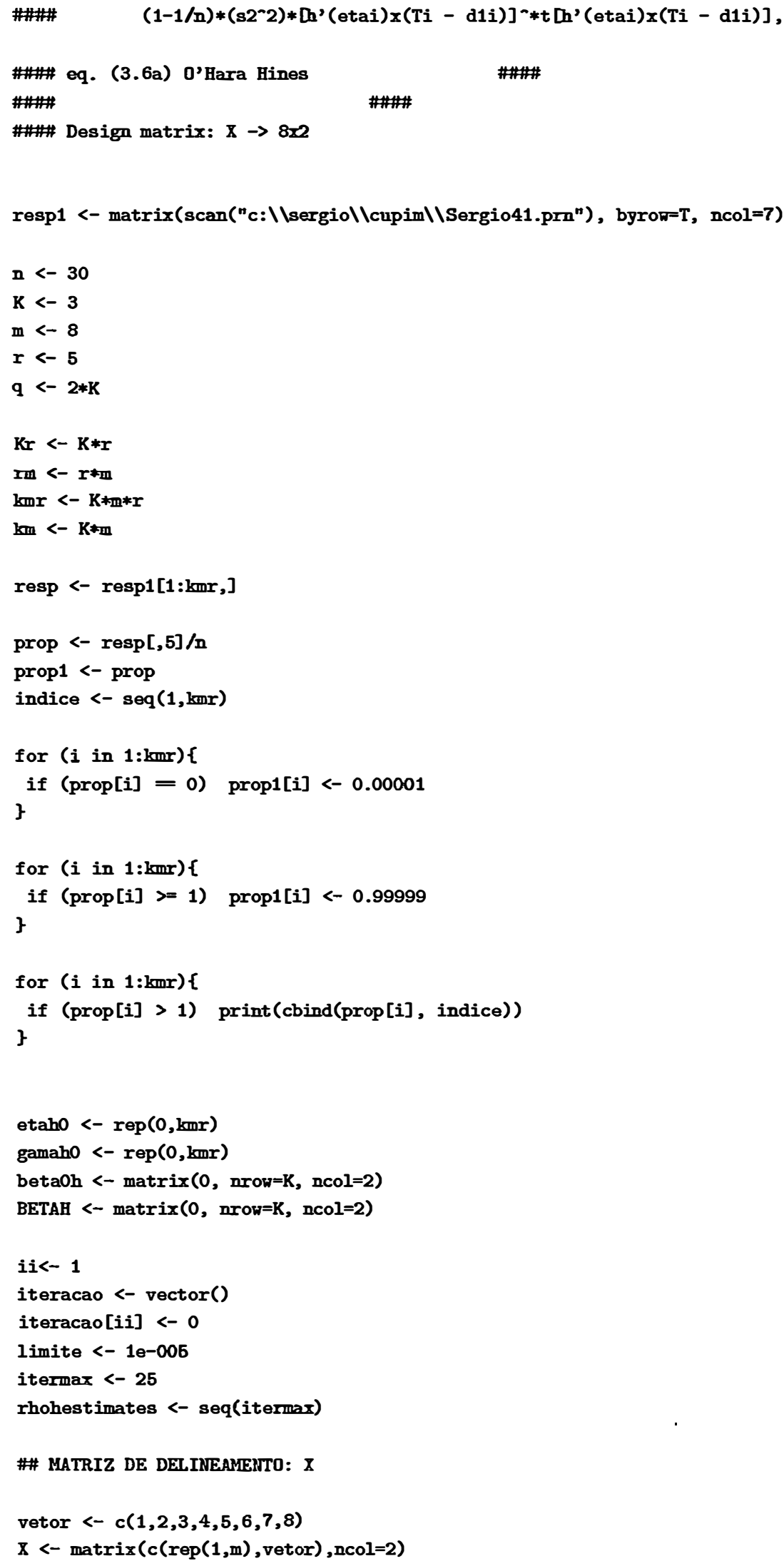




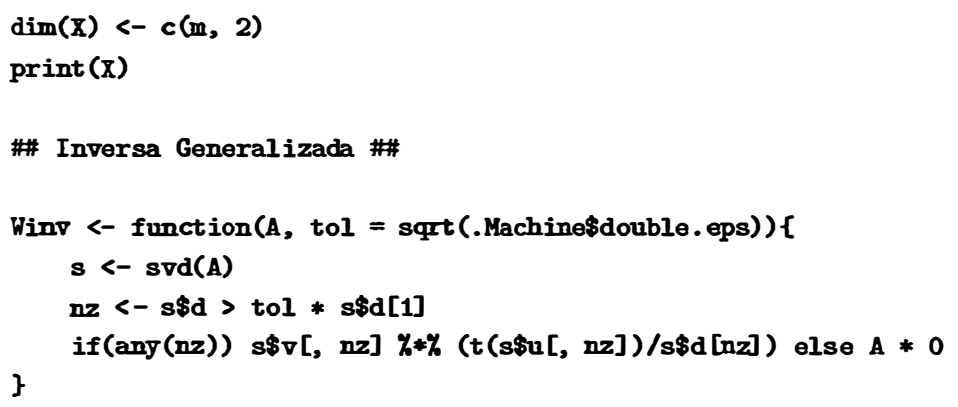




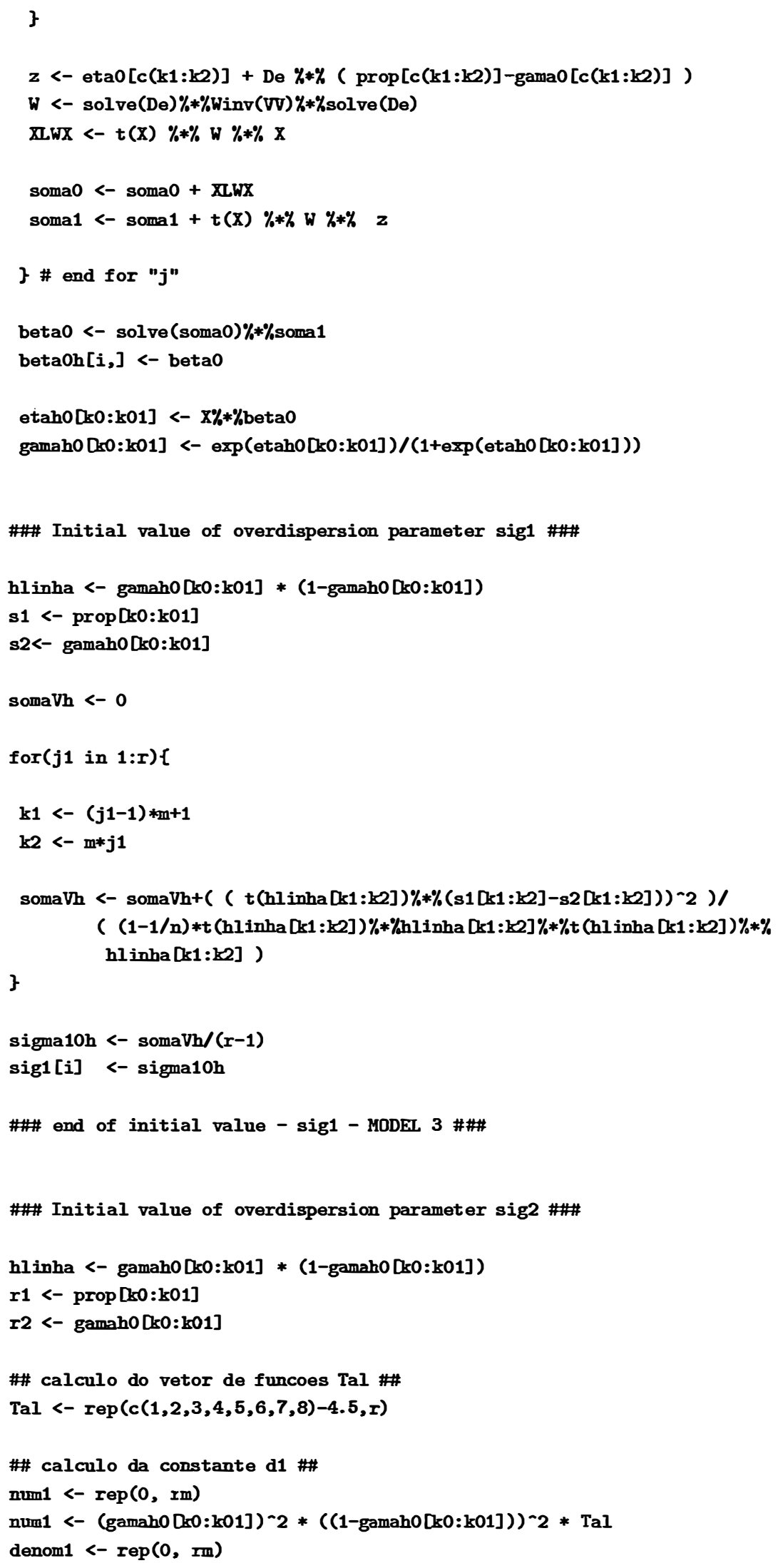




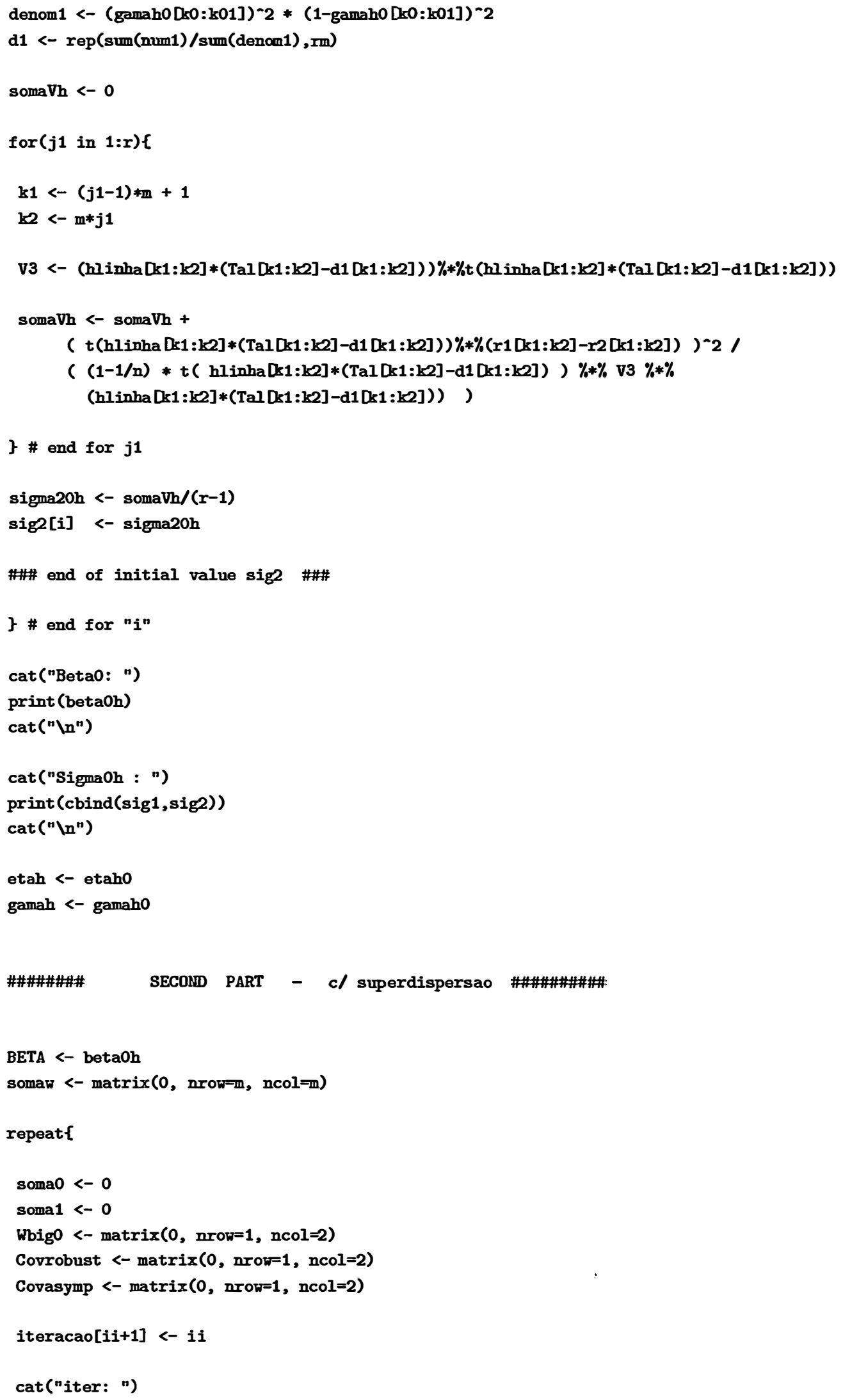




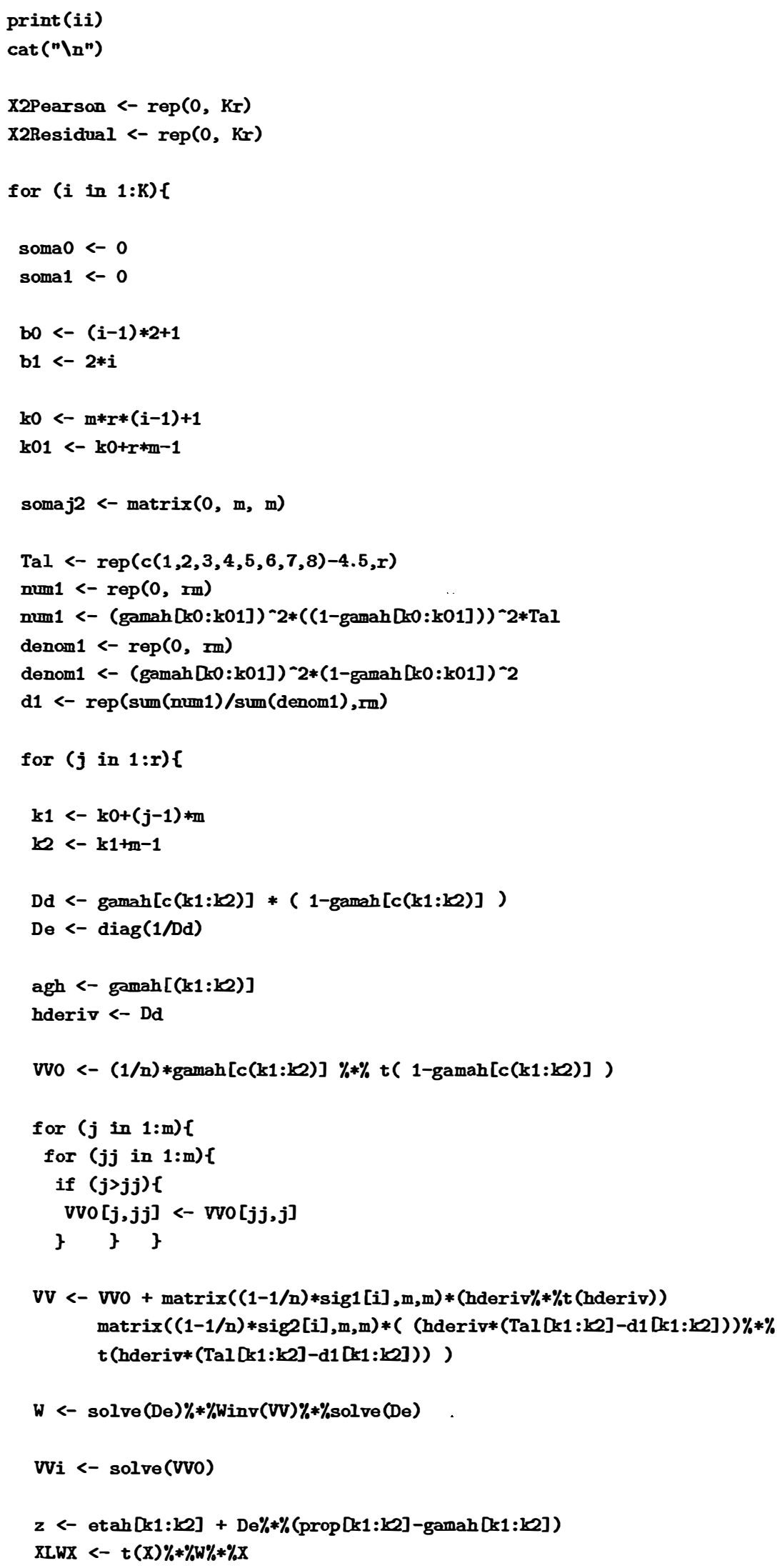




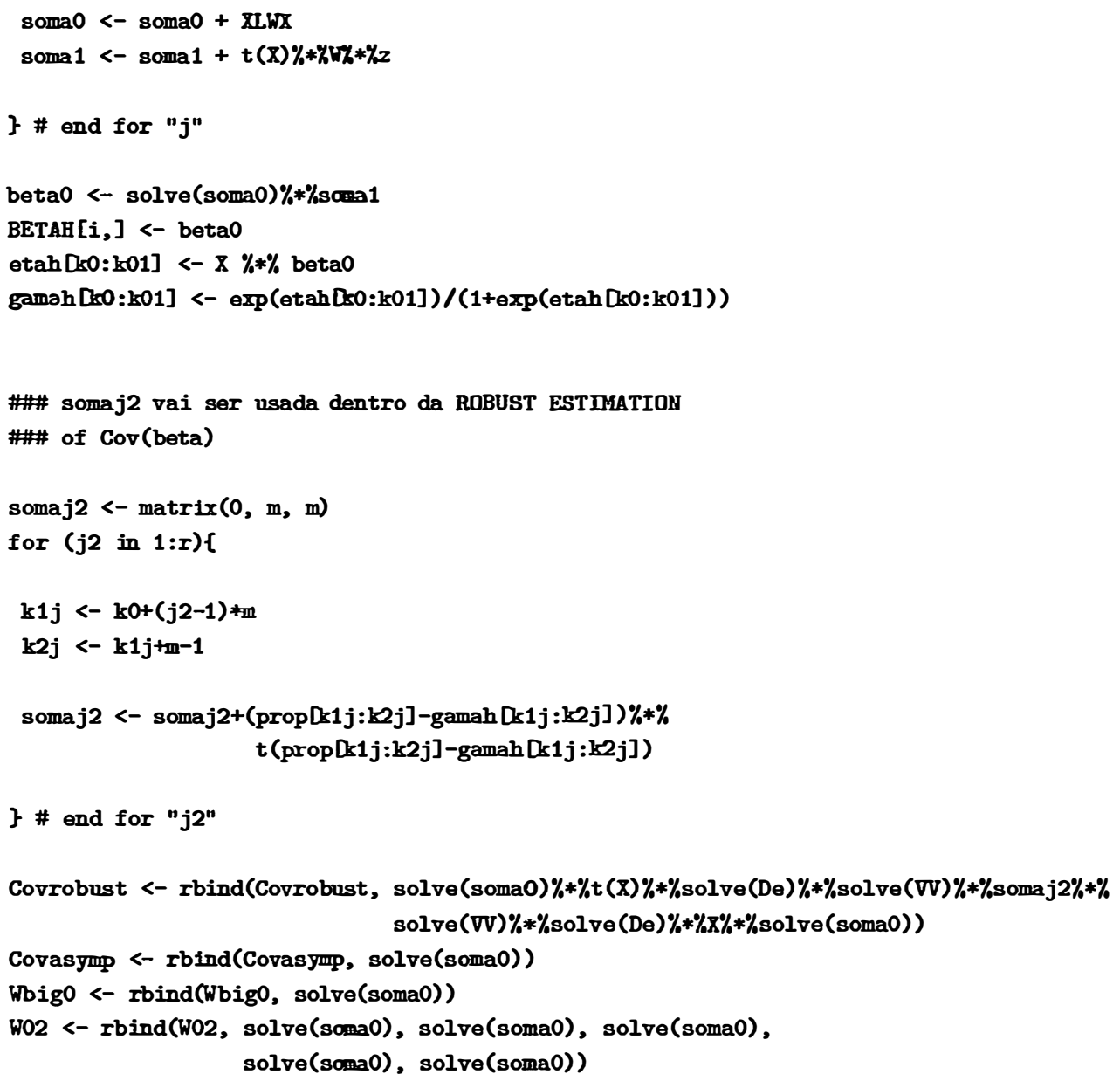




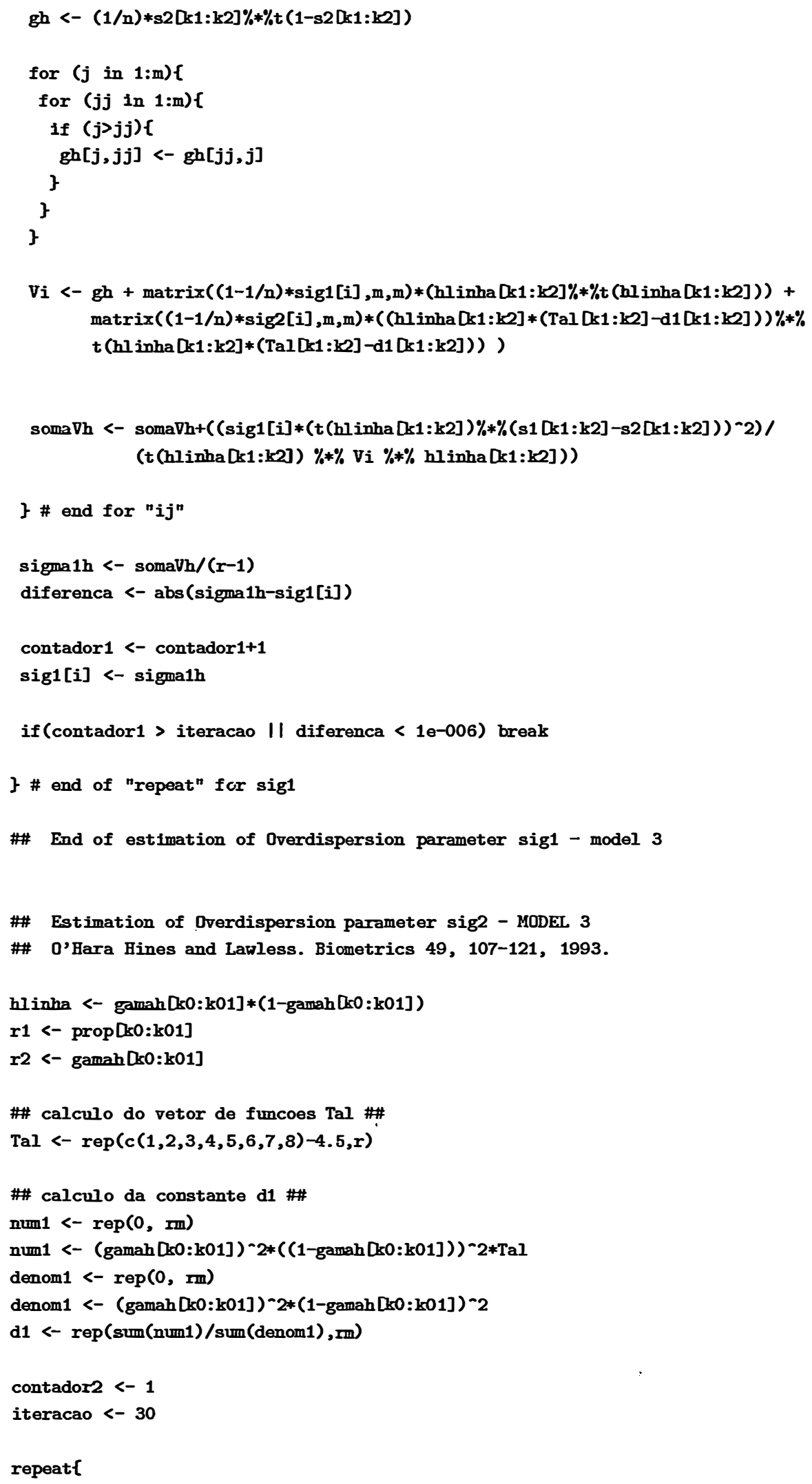




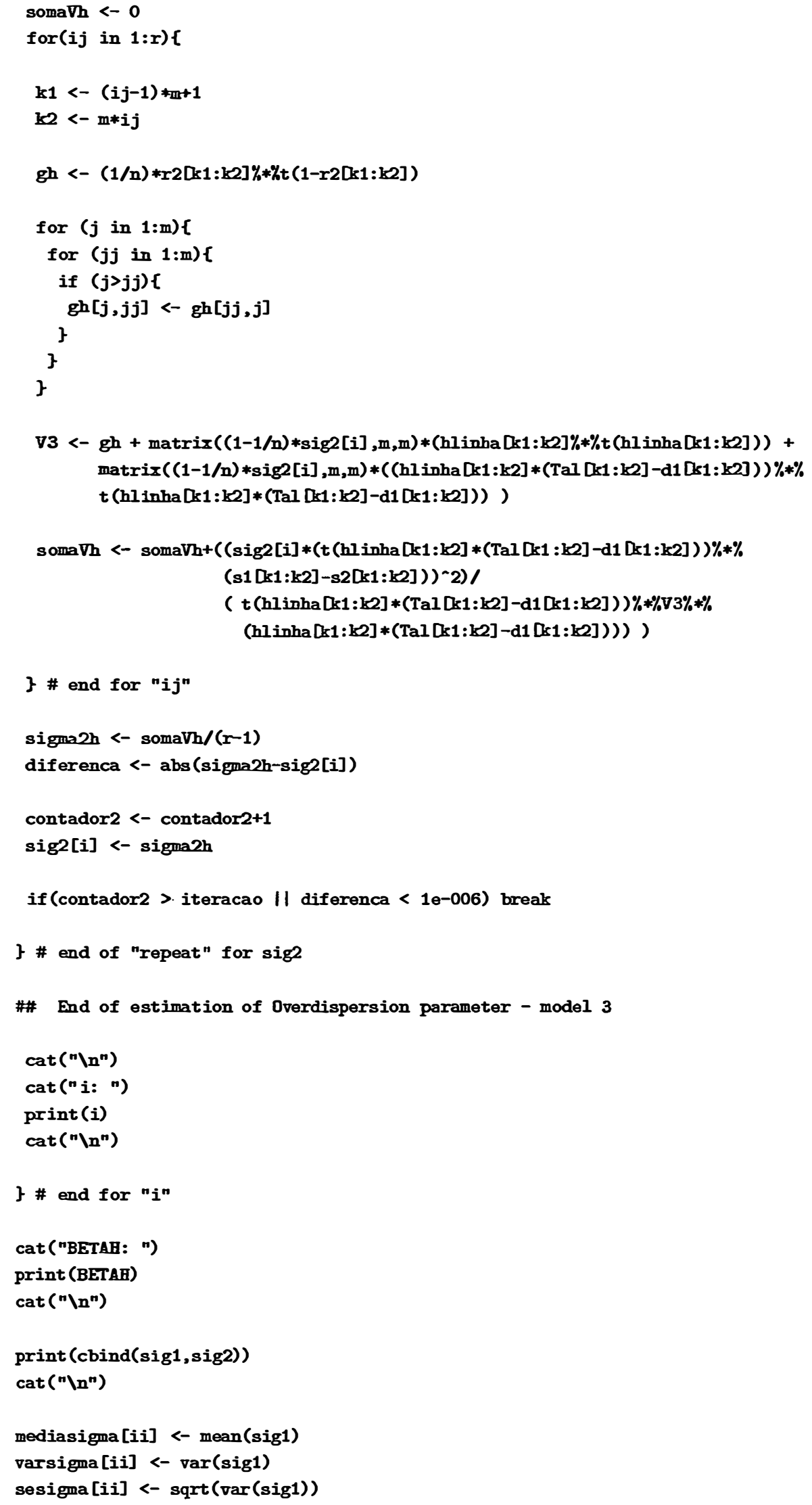




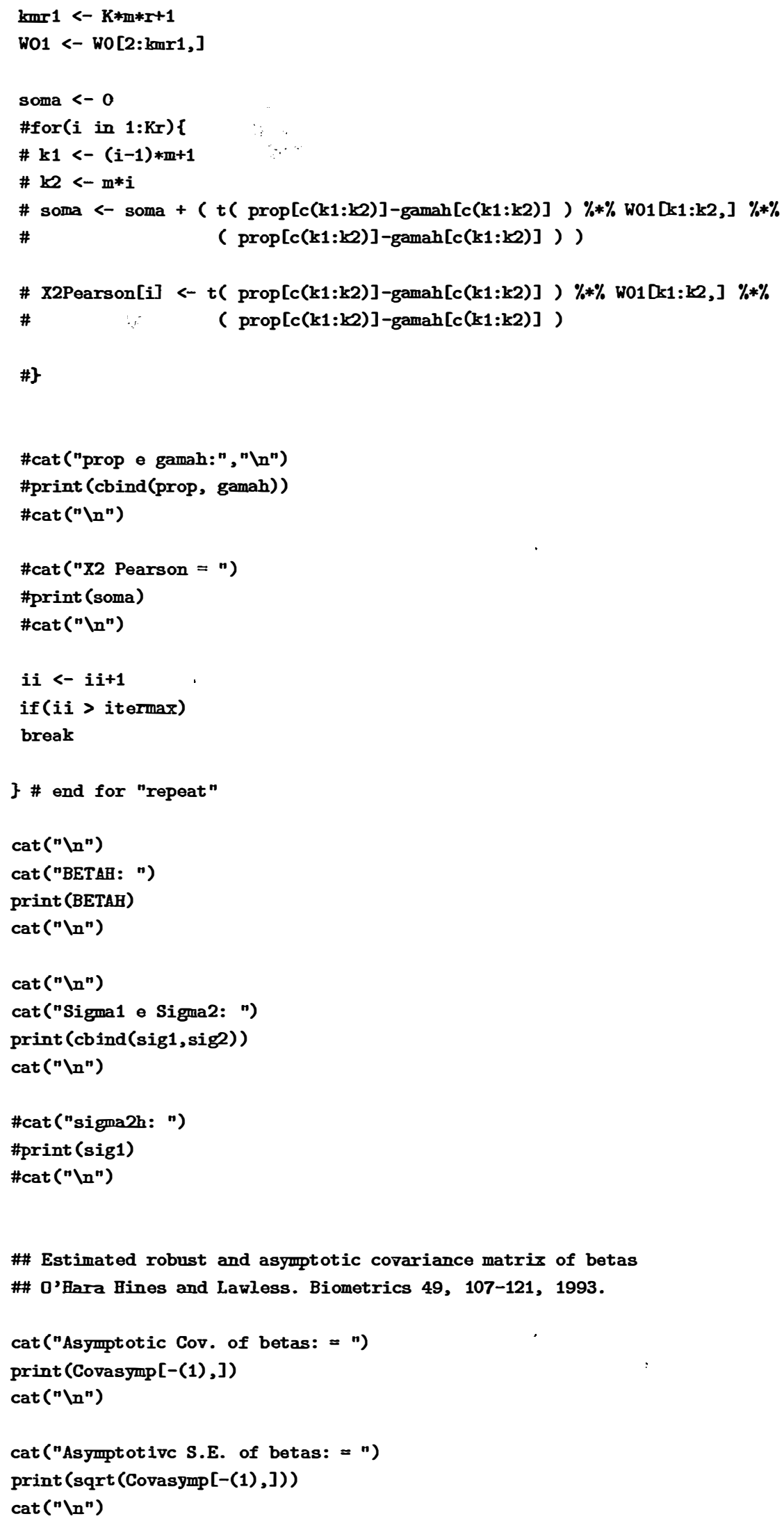

\#) 